\title{
Channel Assignment using Topology Control based on Power Control in Wireless Mesh Networks
}

\author{
by \\ Aizaz Chaudhry, B. Sc. \\ A thesis submitted to the Faculty of Graduate \\ Studies and Research in partial fulfillment of the \\ requirements for the degree of \\ Master of Applied Science in Electrical and \\ Computer Engineering \\ Ottawa-Carleton Institute of Electrical and \\ Computer Engineering (OCIECE) \\ Department of Systems and Computer Engineering \\ Carleton University \\ Ottawa, Ontario, Canada, K1S 5B6
}

August 2010

C Copyright 2010, Aizaz Chaudhry 
Library and Archives

Canada

Published Heritage

Branch

395 Wellington Street

Ottawa ON K1A ON4

Canada
Bibliothèque et

Archives Canada

Direction du

Patrimoine de l'édition

395 , rue Wellington

Ottawa ON K1A ON4

Canada
Your file Votre référence

ISBN: 978-0-494-71526-0

Our file Notre référence

ISBN: 978-0-494-71526-0

\section{NOTICE:}

The author has granted a nonexclusive license allowing Library and Archives Canada to reproduce, publish, archive, preserve, conserve, communicate to the public by telecommunication or on the Internet, loan, distribute and sell theses worldwide, for commercial or noncommercial purposes, in microform, paper, electronic and/or any other formats.

The author retains copyright ownership and moral rights in this thesis. Neither the thesis nor substantial extracts from it may be printed or otherwise reproduced without the author's permission.
AVIS:

L'auteur a accordé une licence non exclusive permettant à la Bibliothèque et Archives Canada de reproduire, publier, archiver, sauvegarder, conserver, transmettre au public par télécommunication ou par l'Internet, prêter, distribuer et vendre des thèses partout dans le monde, à des fins commerciales ou autres, sur support microforme, papier, électronique et/ou autres formats.

L'auteur conserve la propriété du droit d'auteur et des droits moraux qui protège cette thèse. $\mathrm{Ni}$ la thèse ni des extraits substantiels de celle-ci ne doivent être imprimés ou autrement reproduits sans son autorisation.
In compliance with the Canadian Privacy Act some supporting forms may have been removed from this thesis.

While these forms may be included in the document page count, their removal does not represent any loss of content from the thesis.
Conformément à la loi canadienne sur la protection de la vie privée, quelques formulaires secondaires ont été enlevés de cette thèse.

Bien que ces formulaires aient inclus dans la pagination, il n'y aura aucun contenu manquant.

\section{Canadä}




\begin{abstract}
Single-radio mesh routers operating on a single channel suffer from low throughput due to collisions. Equipping mesh routers with multiple radios operating on non-overlapping channels can significantly improve the end-to-end throughput. However, the number of available non-overlapping channels is limited and the assignment of channels to radios is a challenging task.

The goal of the channel assignment algorithm proposed in this thesis, TICA (Topology-controlled Interference-aware Channel-assignment Algorithm), is to improve the network throughput in a multi-radio multi-channel wireless mesh network by minimizing interference within the mesh network by intelligently controlling the network topology based on power control as well as guaranteeing network connectivity before efficiently assigning channels to multi-radio mesh routers. In addition to channel assignment, TICA also performs routing. It is fault-tolerant and supports automatic and fast failure recovery.

The performance of TICA is compared with two commonly-used benchmark schemes and significant improvements are shown through simulations in both small and large networks. TICA has also been shown to achieve better performance than two wellknown centralized channel assignment schemes.
\end{abstract}




\section{Acknowledgements}

I am truly thankful to Allah, my creator, who gave me the determination and strength to enable me to complete this work. I am deeply thankful to my supervisor Professor Roshdy H. M. Hafez for his kind and continuous support, without which this work would not have been possible. I am grateful to him for his patience and feedback, which helped me to improve the quality of my work. I am also deeply grateful to Dr. Osama Aboul-Magd, who was always there for me when I needed advice, guidance or feedback for my work. His encouragement kept me focused and his support encouraged me to work hard to the best of my ability. I am also thankful to the defence committee members, Professor Halim Yanikomeroglu and Professor Claude D'Amours, for their comments and feedback, which greatly helped me to improve the quality of presentation of this thesis. I am thankful to my colleagues Talha Ahmad, Muhammad Ajmal Khan and Ghassan Dahman, who provided me with valuable feedback for my work. I deeply appreciate their help in proofreading my thesis and manuscripts before submission.

I must thank my parents, especially my mother for her prayers as they have helped me to achieve the milestones related to this work and my life, in general, which I thought were not possible. Finally, I must thank my wife for her understanding and support during the long and odd hours of my research work. 


\section{TABLE OF CONTENTS}

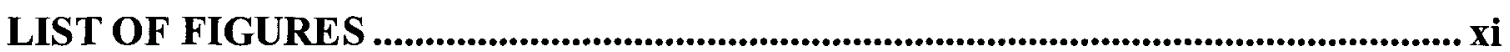

LIST OF TABLES ....................................................................................................

LIST OF ACRONYMS AND ABBREVIATIONS ................................................. xv

CHAPTER 1

INTRODUCTION

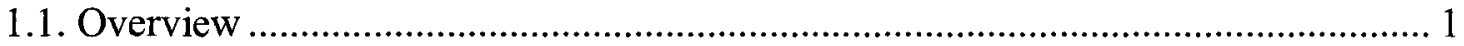

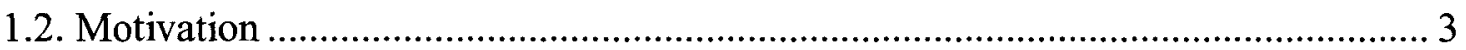

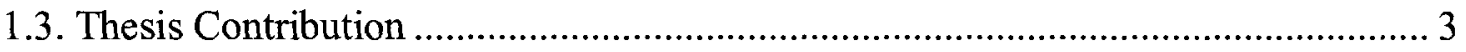

1.4. Thesis Organization......................................................................................... 4

\section{CHAPTER 2}

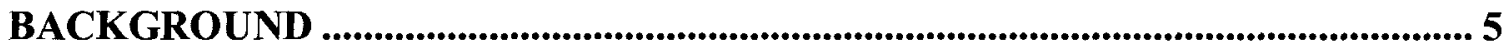

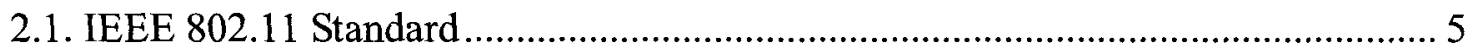

2.1.1. Physical Layer Transmission Technologies ............................................... 6

2.1.2. Medium Access Control Layer................................................................ 7

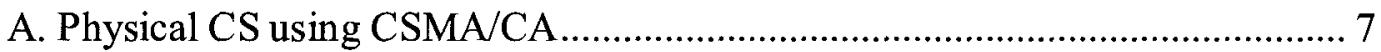

B. Virtual CS using Request to Send and Clear to Send ........................................ 7

2.1.2. IEEE 802.11a

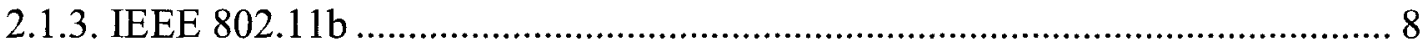

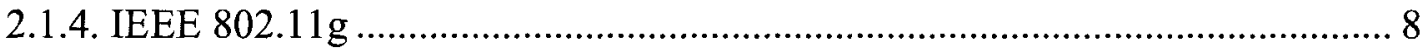

2.2. IEEE 802.1 1s for Wireless Mesh Networks .................................................... 8

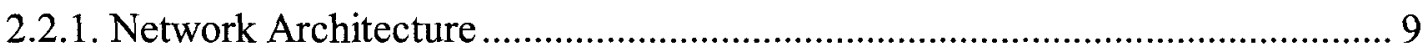

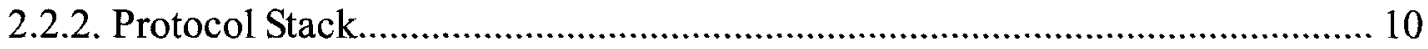

2.3. Topology Control Schemes .......................................................................... 10

2.3.1. Topology Control in Multi-radio WMNs .................................................... 11

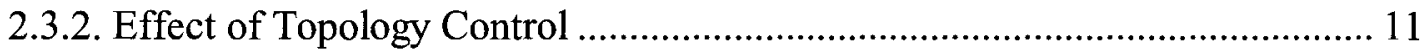

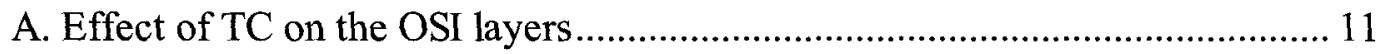

B. Effect of TC on the Performance of the Network ............................................ 12

C. Effect of TC on the Performance of the MAC and Routing Protocols ............. 12

2.3.3. Guidelines for an Effective Topology Control Algorithm .............................. 12

2.3.4. Taxonomy of Topology Control Schemes ................................................. 13

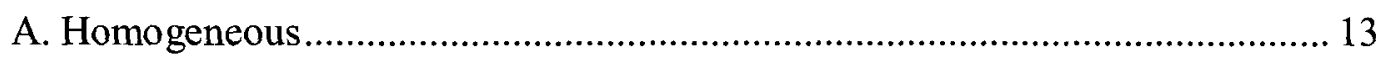

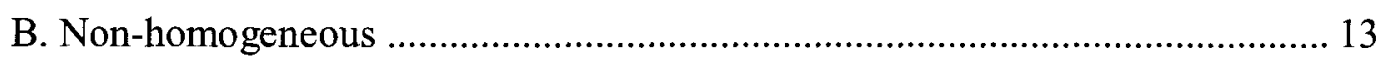

2.3.5. Related Topology Control Algorithms ......................................................... 14 
A. Local Minimum Spanning Tree Algorithm .............................................. 14

B. Enhanced Local Minimum Shortest Path Tree Algorithm............................ 14

C. Maximum Minimum Power Algorithm ................................................... 15

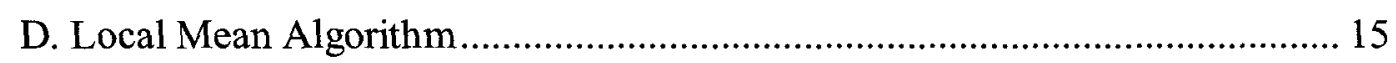

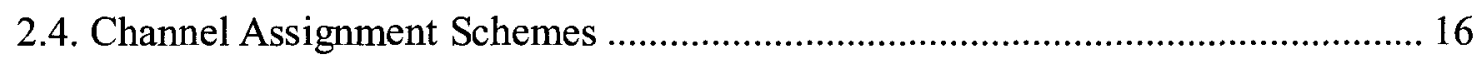

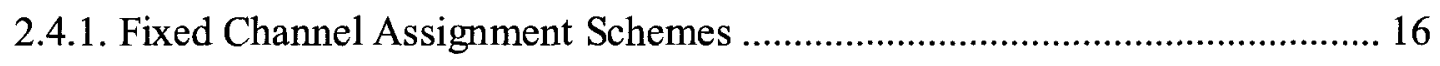

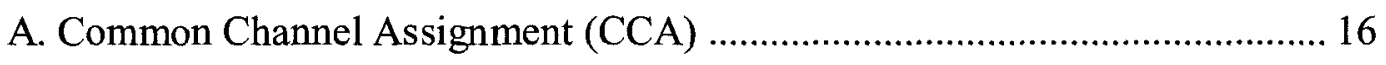

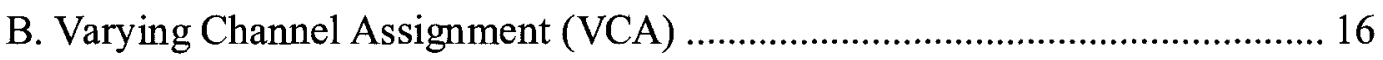

2.4.2. Dynamic Channel Assignment Schemes .................................................... 18

A. Multi-channel Medium Access Control (MMAC) ....................................... 18

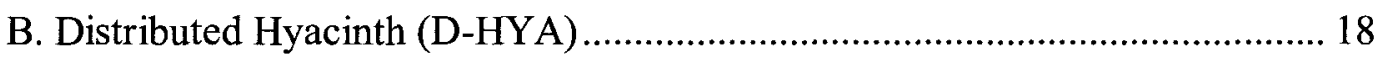

2.4.3. Hybrid Channel Assignment Schemes ......................................................... 19

A. Hybrid Multi-Channel Protocol (HMCP) .................................................... 19

B. Breadth First Search - Channel Assignment (BFS-CA)................................ 19

\section{CHAPTER 3}

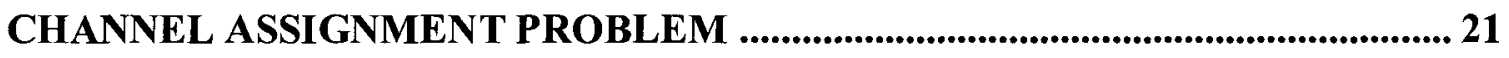

3.1. IEEE 802.11 Medium Access Issues............................................................. 21

3.1.1. Hidden and Exposed Terminal Problems ................................................... 21

3.1.2. Single-radio Single-channel Mesh Network ............................................. 22

3.1.3. Dual-radio Single-channel Mesh Network .................................................. 22

3.1.4. Multi-radio Multi-channel Mesh Network .................................................. 22

3.2. Connectivity and Interference Trade-off....................................................... 23

3.2.1. Maximum Connectivity in a Single-radio Single-channel Scenario ............... 24

3.2.2. Maximum Connectivity in a Multi-radio Multi-channel Scenario................... 24

3.2.3. Minimum Interference in a Multi-radio Multi-channel Scenario ................... 24

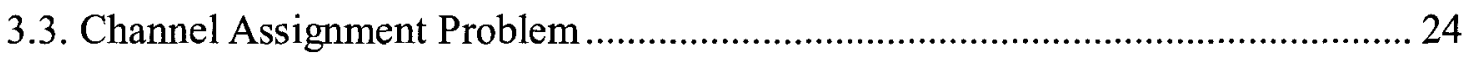

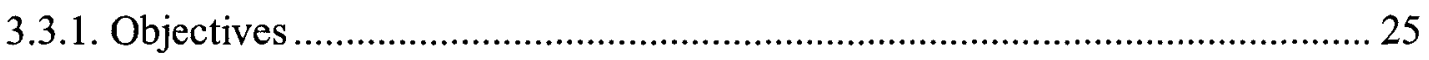

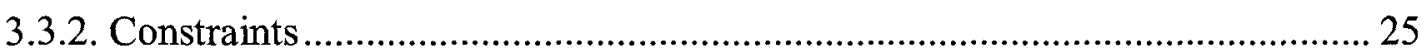

\section{CHAPTER 4}

TOPOLOGY CONTROL AND CHANNEL ASSIGNMENT ALGORITHMS....... 26

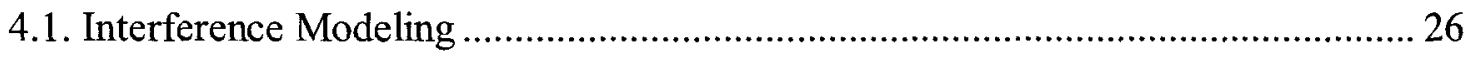

4.1.1. Graph Theory Representation of a WMN ................................................. 26

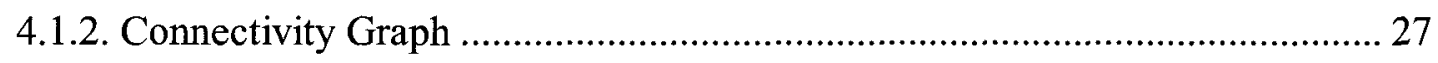

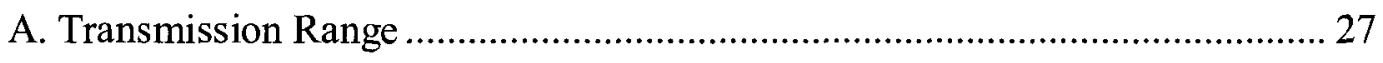




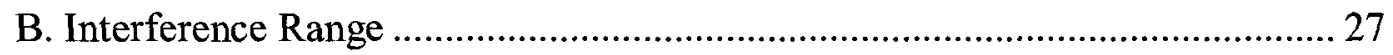

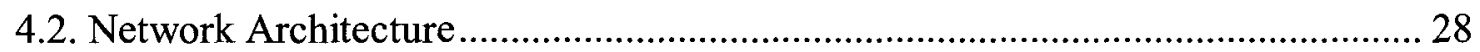

4.3. Topology Control Algorithm .................................................................... 28

4.3.1. Gateway Advertisement Process ............................................................ 29

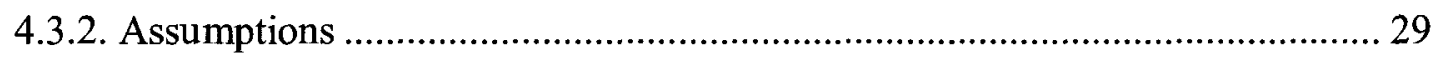

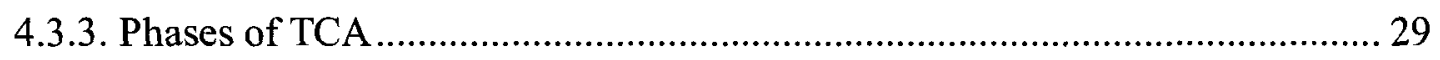

A. Exchange of Information Between Nodes .............................................. 29

B. Building the Maximum Power Neighbor Table (MPNT) ............................. 29

C. Building the Direct Neighbor Table (DNT) ............................................. 30

D. Converting into Bi-directional Links .................................................... 30

E. Calculating the Minimum Power Required ................................................ 30

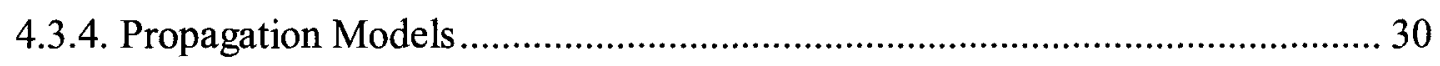

4.4. Channel Assignment Algorithm............................................................. 31

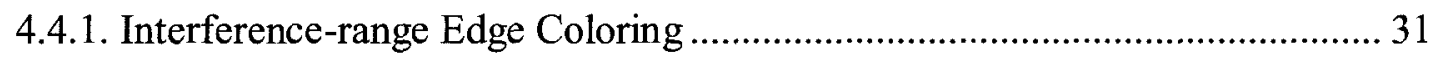

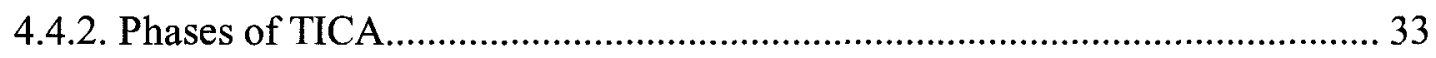

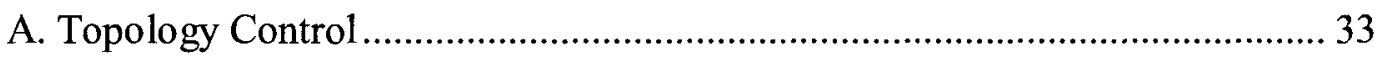

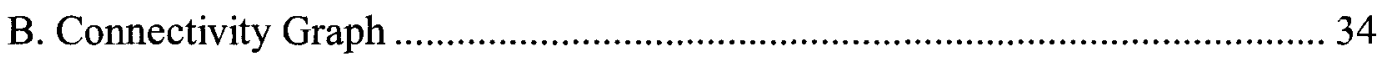

C. Minimum Power-based Shortest Path Tree with a MND of Four................... 34

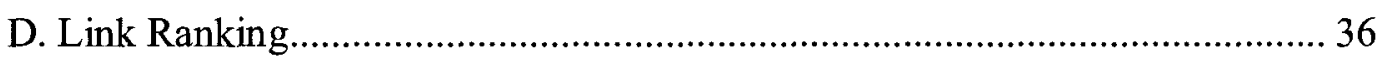

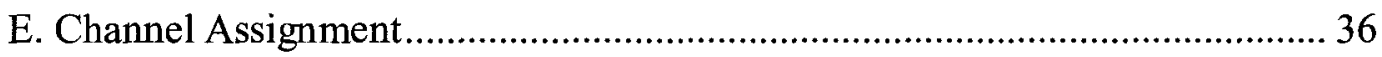

4.5. Failure Recovery Mechanism of TICA ........................................................... 38

A. Periodic Keep-Alive Messages..................................................................... 38

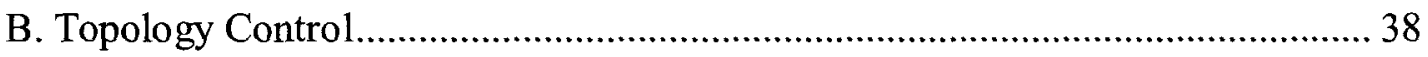

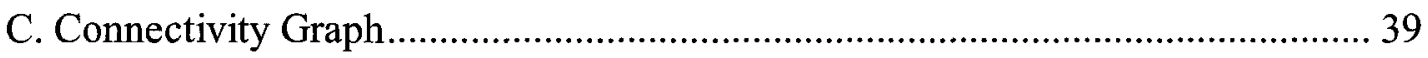

D. Minimum Power-based SPT with a MND of Four.......................................... 39

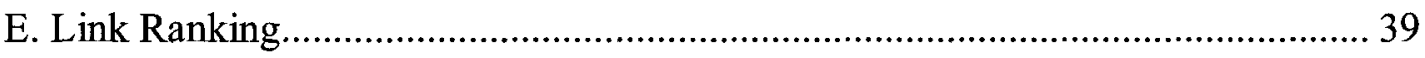

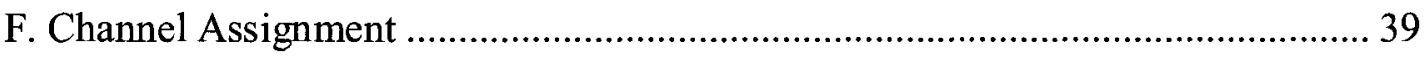

\section{CHAPTER 5}

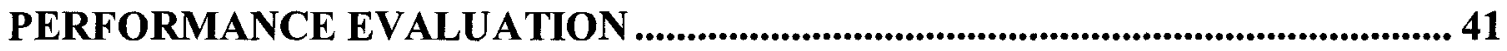

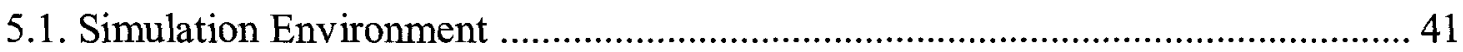

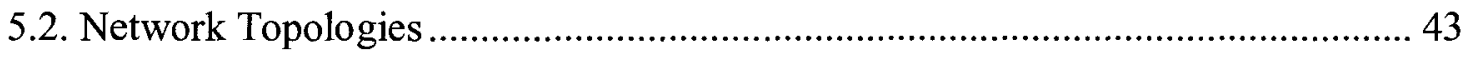

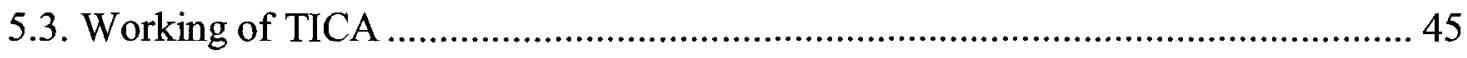

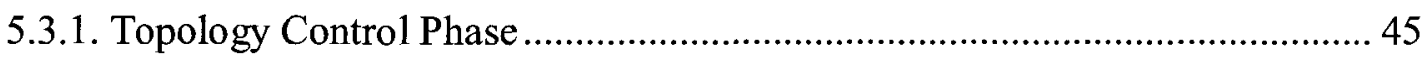

A. Exchanging Hello Messages .............................................................. 45 


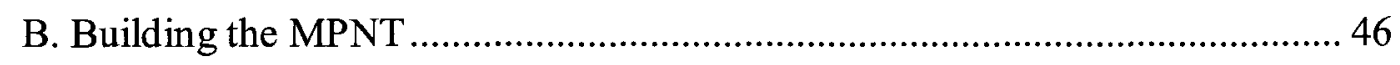

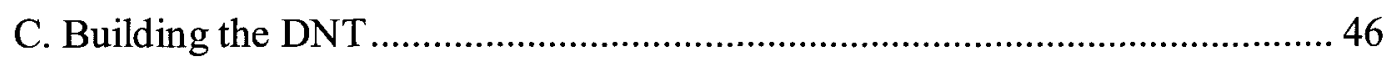

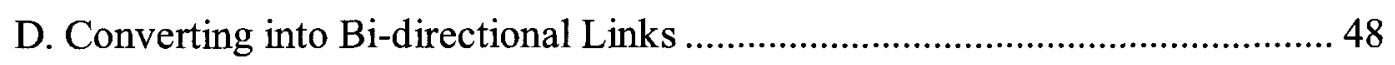

E. Calculating the Minimum Power Required .................................................. 49

5.3.2. Connectivity Graph ................................................................................... 50

A. Connectivity Graph for Select 1 for Less Than 1 TCA ………………............ 50

B. Connectivity Graph for Select 2 for Less Than 2 TCA..................................... 50

C. Connectivity Graph for Select 3 for Less Than 3 TCA.................................... 50

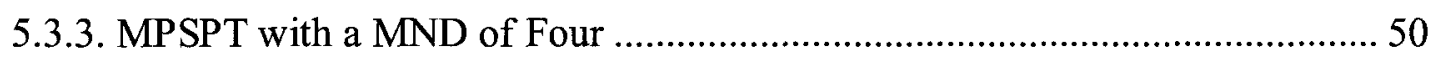

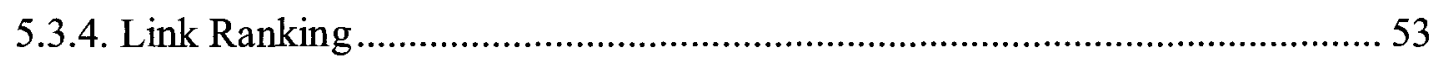

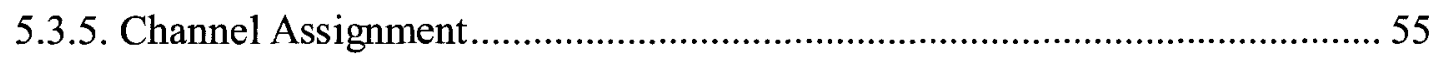

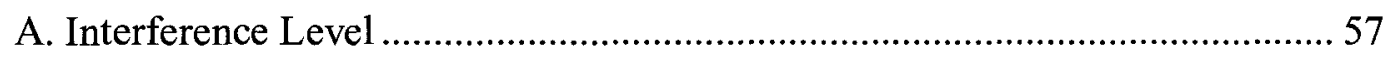

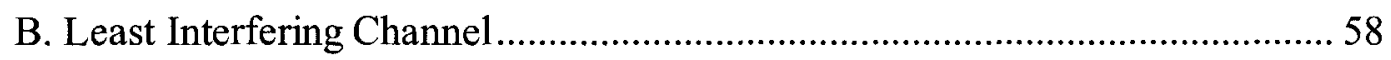

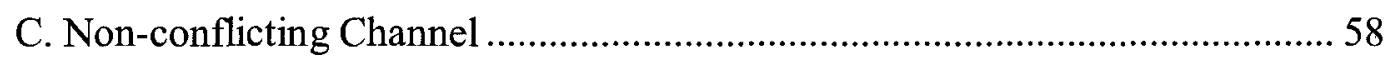

D. Channel Assignment and Routing Message ................................................... 59

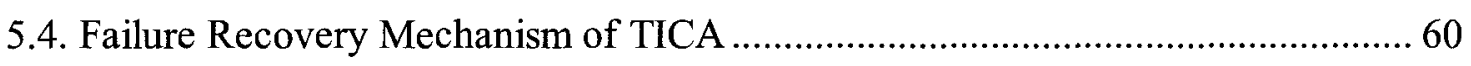

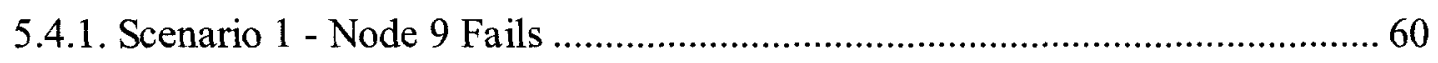

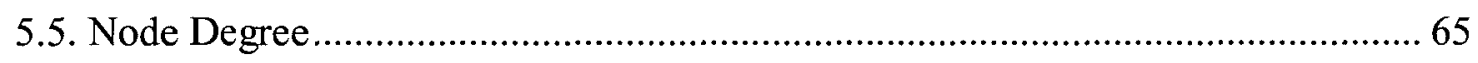

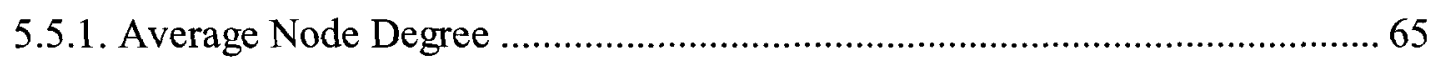

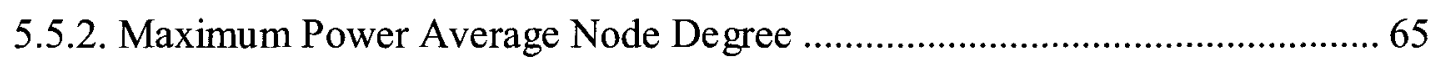

A. Maximum Power TR Average Node Degree ………………………............... 65

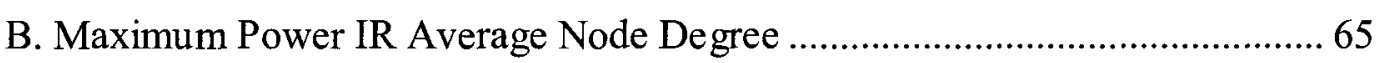

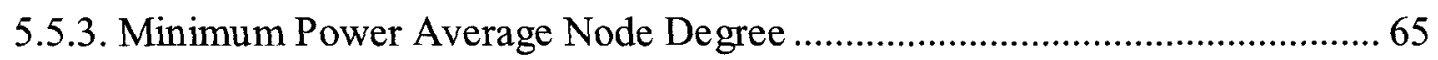

A. Minimum Power TR Average Node Degree.................................................. 65

B. Minimum Power IR Average Node Degree ………………………………........ 65

\section{CHAPTER 6}

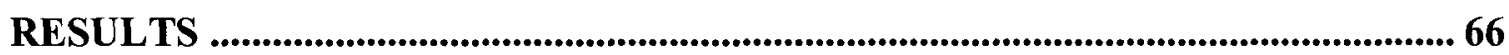

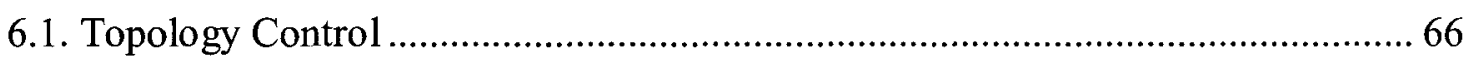

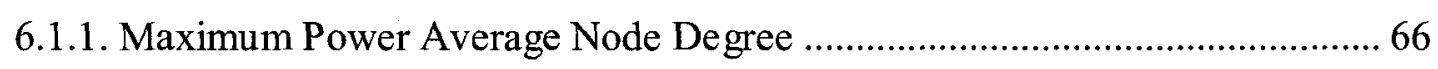

6.1.2. Minimum Power Average Node Degree ......................................................... 67

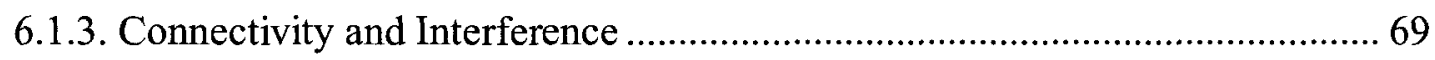

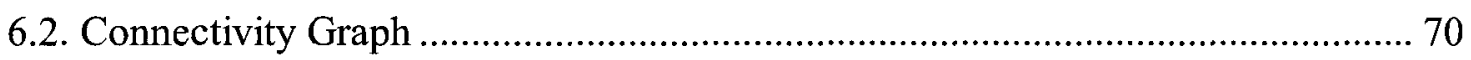

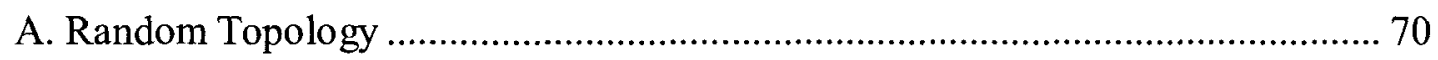

B. Controlled Random Topology ………………………................................. 70 


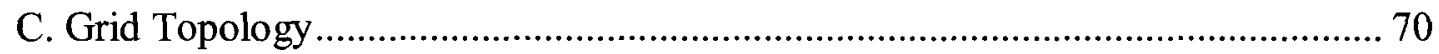

6.3. Minimum Power-based Shortest Path Tree.................................................... 70

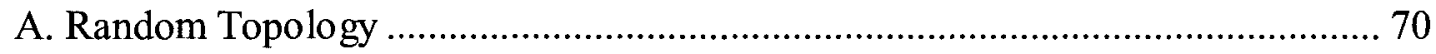

B. Controlled Random Topology ………………………................................ 70

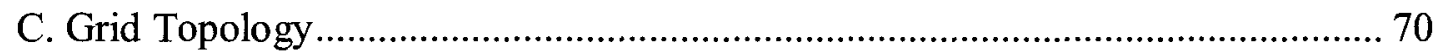

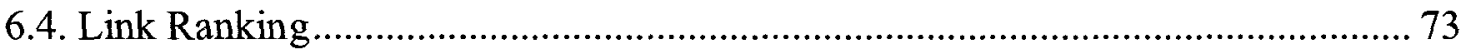

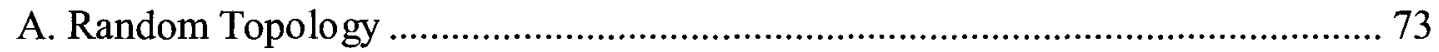

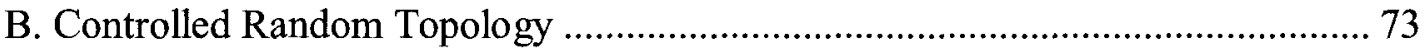

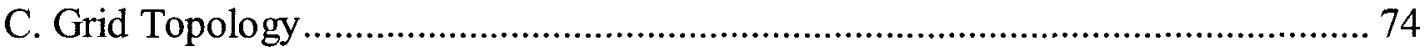

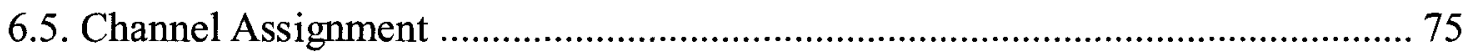

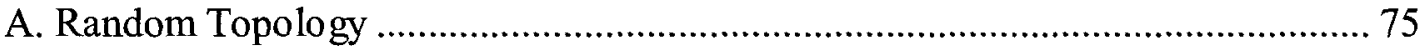

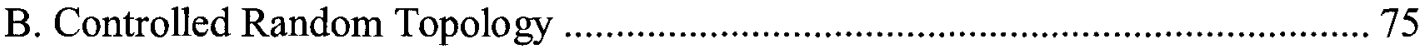

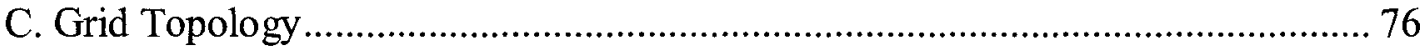

6.6. Simulation Results in NS2 based on Throughput Analysis .................................. 77

6.6.1. Simulation Parameters.............................................................................. 77

6.6.2. Simulation Results for a 36-node Network ................................................... 80

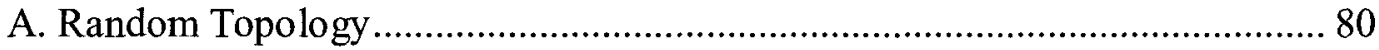

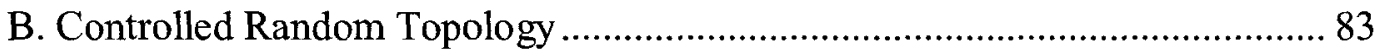

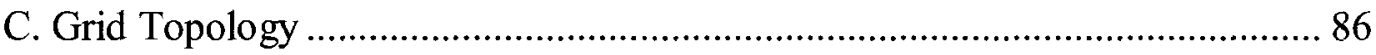

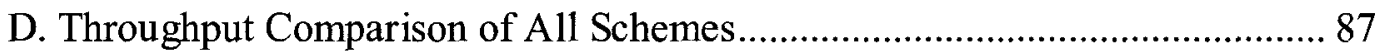

6.6.3. Simulation Results for a 144-node Network ………………………………... 88

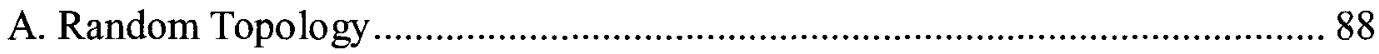

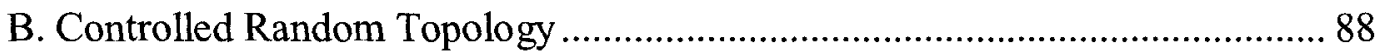

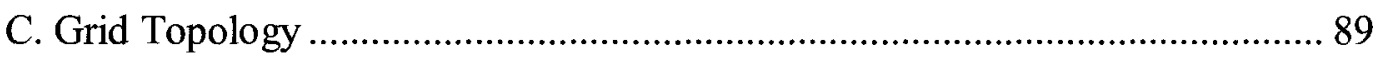

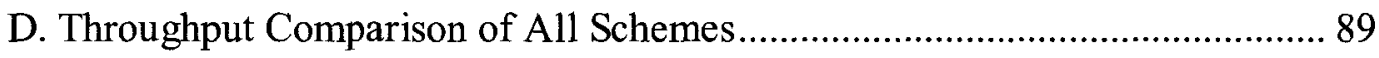

E. Throughput Analysis for the Three Topologies ............................................. 90

6.6.4. Performance Comparison of TICA with Centralized CA Schemes ................. 91

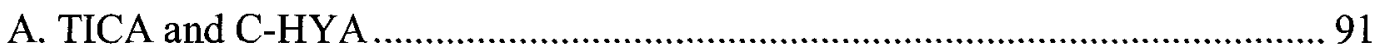

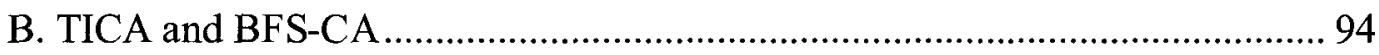

6.7. Features' Comparison of Related CA Schemes ...................................................... 97

\section{CHAPTER 7}

CONCLUSION AND FUTURE WORK ...................................................................99

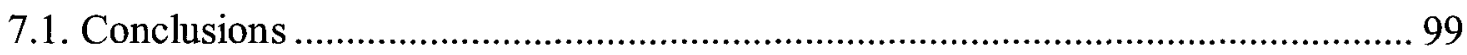

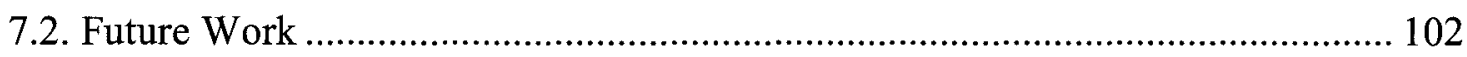




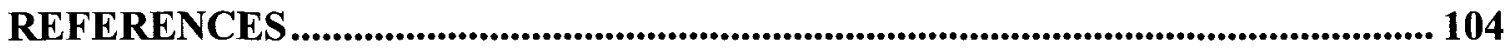

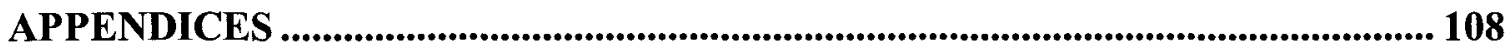

Appendix A. Final Neighbor Table (FNT) …………………………………......... 108

A.1. FNTs from Select 1 for Less Than 1 TCA …………..................................... 108

A.2. FNTs from Select 2 for Less Than 2 TCA ……………………………….... 109

A.3. FNTs from Select 3 for Less Than 3 TCA ………......................................... 110

Appendix B. Failure Recovery Mechanism of TICA ………………….................... 111

B.1. Scenario 2 - Node 3 Fails.............................................................................. 111

B.2. Scenario 3 - Node 30 Fails........................................................................ 115

B.3. Scenario 4 - Node 5 Fails................................................................................. 118

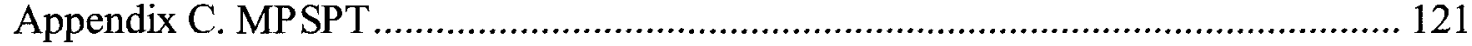

C.1. MPSPT for Random Topology - 36-node Network ......................................... 121

C.2. MPSPT for Controlled Random Topology - 36-node Network ....................... 126

C.3. MPSPT - 144-node Network..................................................................... 132

C.4. MPSPT - 100-node Grid Network ………………..................................... 134

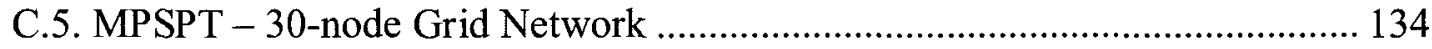




\section{LIST OF FIGURES}

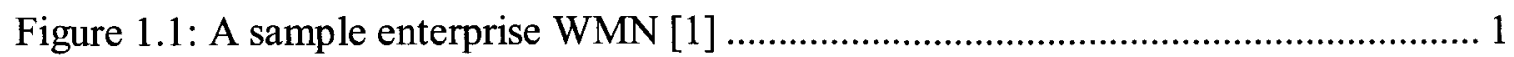

Figure 2.1: Available channels for DSSS in the $2.4 \mathrm{GHz}$ ISM band [4] ......................... 6

Figure 2.2: IEEE 802.11s Mesh Network Architecture [8] ............................................ 9

Figure 2.3: IEEE 802.11s Protocol Stack [8] .......................................................... 10

Figure 2.4: ELMST (a) before topology control (b) after topology control [11] ............. 15

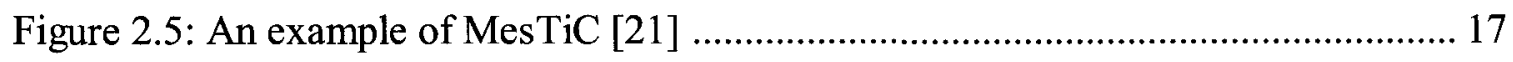

Figure 3.1: Hidden and exposed terminal problems [1] ………................................. 21

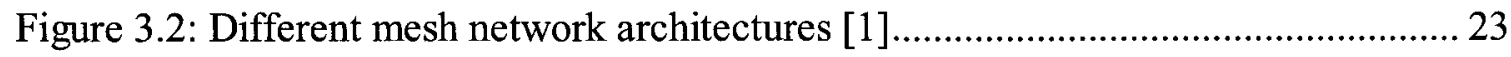

Figure 3.3: Trade-off between connectivity and interference [19] ................................ 24

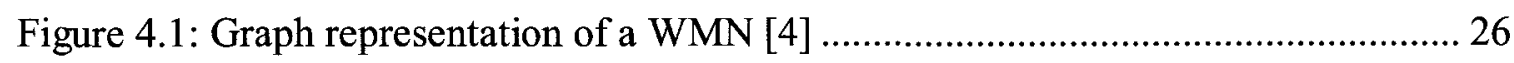

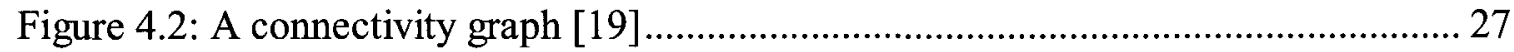

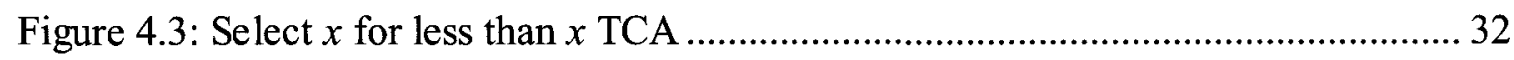

Figure 4.4: Two edges at distance-2 of each other [30].................................................. 33

Figure 4.5: Interference-range edge coloring............................................................. 33

Figure 4.6: Topology-controlled Interference-aware Channel-assignment Algorithm

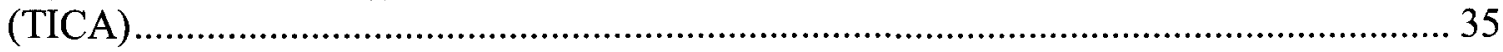

Figure 4.7: Failure Recovery Mechanism of TICA ……….......................................... 40

Figure 5.1: Grid Topology ..................................................................................... 43

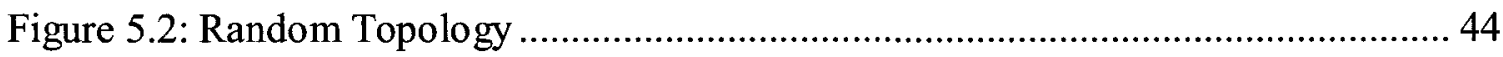

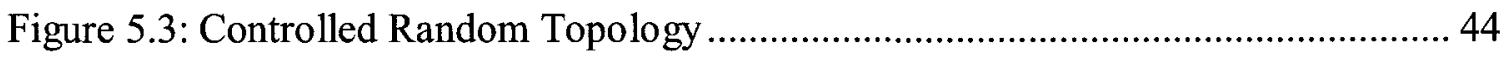

Figure 5.4: Maximum Power Transmission Range neighbors of Node 1....................... 45

Figure 5.5: Connectivity Graph for Select 1 for less than 1 TCA …….......................... 51

Figure 5.6: Connectivity Graph for Select 2 for less than 2 TCA ................................... 51

Figure 5.7: Connectivity Graph for Select 3 for less than 3 TCA ……......................... 52

Figure 5.8: MPSPT with a MND of four for Select 3 for less than 3 TCA ..................... 52

Figure 5.9: Channel-assigned links within IR of Node 10............................................. 56

Figure 5.10: Channel-assigned links within IR of Node 27 ...........................................56

Figure 5.11: Connectivity Graph with Select 3 for less than 3 TCA after Node 9 fails ... 61

Figure 5.12: Connectivity Graph with Select 4 for less than 4 TCA after Node 9 fails ... 61

Figure 5.13: MPSPT with a MND of 4 after Node 9 fails ................................................. 62

Figure 6.1: Minimum Power TR Average Node Degree................................................ 67

Figure 6.2: Minimum Power IR Average Node Degree ....................................................6 68

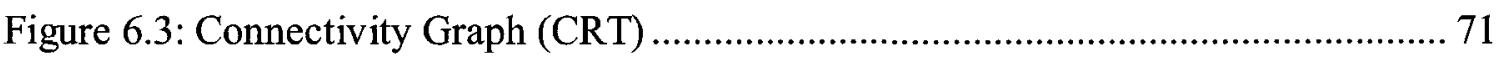

Figure 6.4: Connectivity Graph (GT) ................................................................. 71

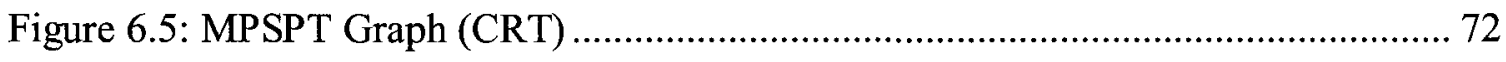

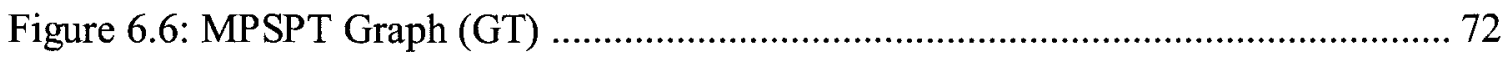

Figure 6.7: AT for Ten Random Topologies - 36-node network ……………................ 81 
Figure 6.8: AT for Ten Controlled Random Topologies - 36-node network .................... 85

Figure 6.9: AT for all schemes - 36-node network .................................................... 87

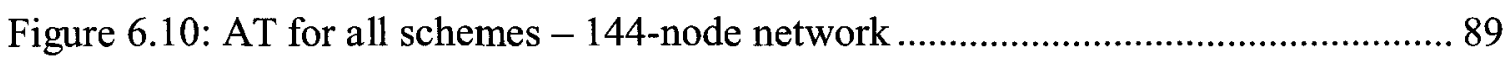

Figure 6.11: Normalized Throughput with ten different TPs .......................................... 93

Figure 6.12: Normalized Throughput with ten different TPs ......................................... 96

Figure B.1: Connectivity Graph with Select 3 for less than 3 TCA after Node 3 fails .. 112

Figure B.2: MPSPT with a MND of 4 after Node 3 fails ............................................ 112

Figure B.3: Connectivity Graph with Select 3 for less than 3 TCA after Node 30 fails 115

Figure B.4: MPSPT with a MND of 4 after Node 30 fails ......................................... 116

Figure B.5: Connectivity Graph with Select 3 for less than 3 TCA after Node 5 fails .. 118

Figure B.6: MPSPT with a MND of 4 after Node 5 fails ............................................ 119

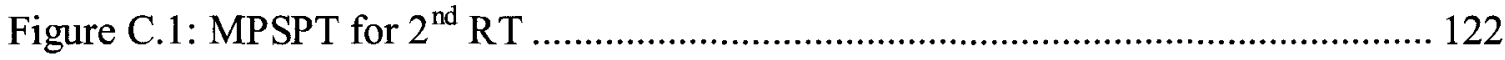

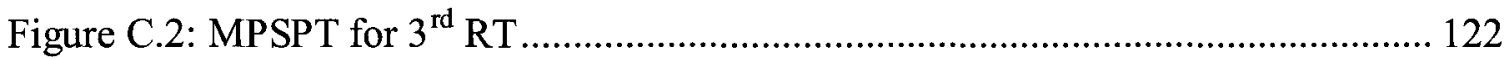

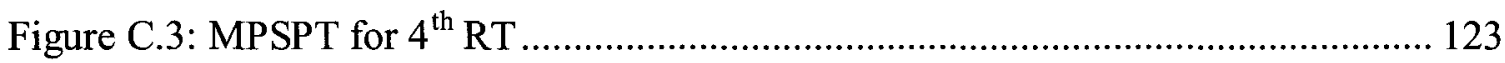

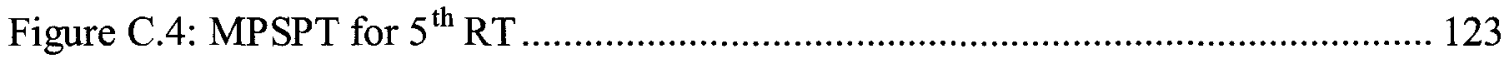

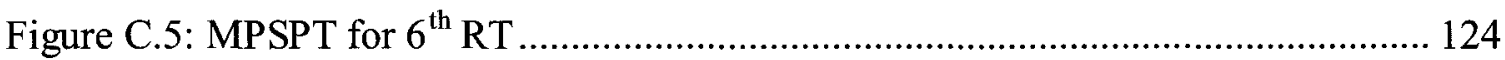

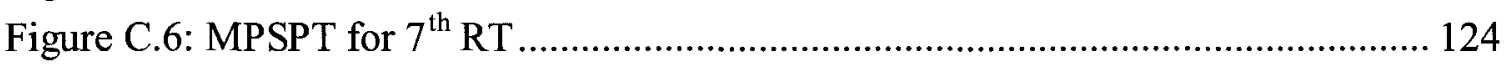

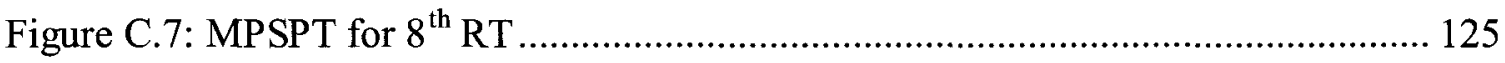

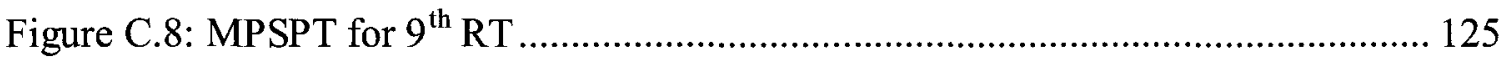

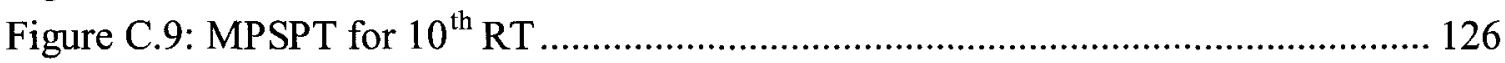

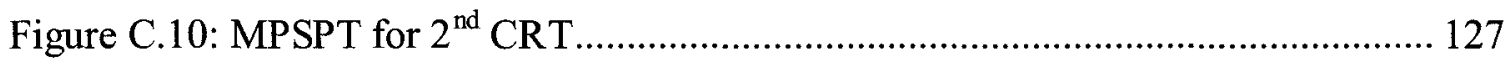

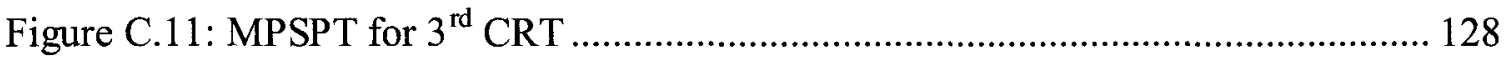

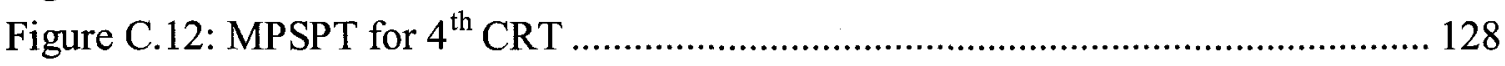

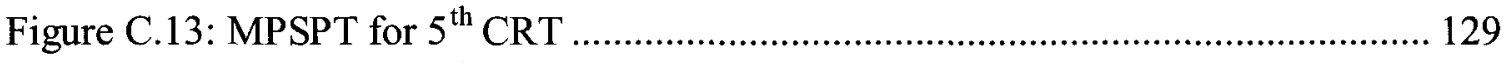

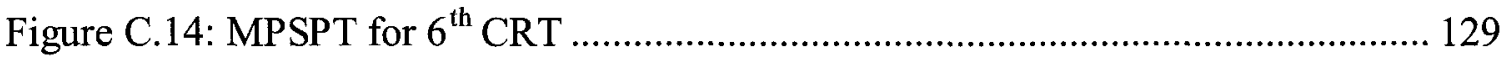

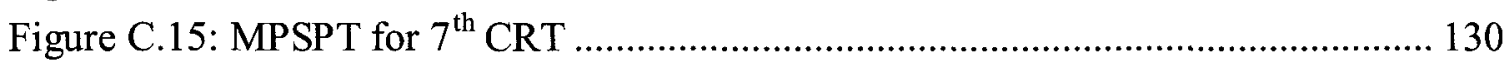

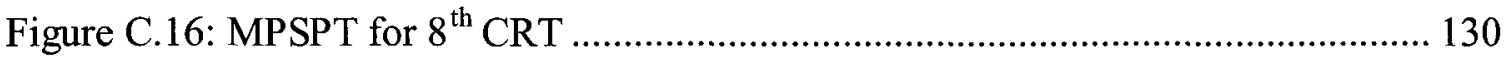

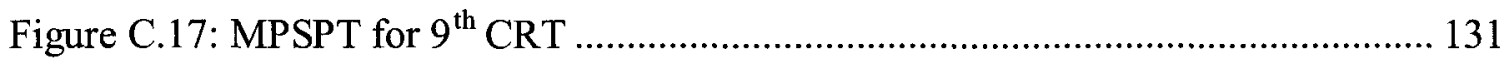

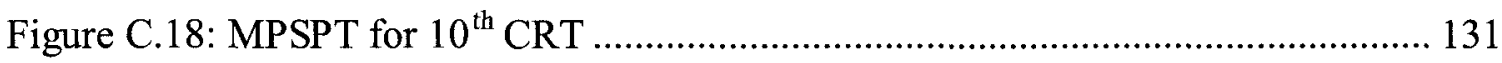

Figure C.19: MPSPT for RT - 144-node network.................................................. 132

Figure C.20: MPSPT for CRT - 144-node network ................................................ 133

Figure C.21: MPSPT for GT - 144-node network …………..................................... 133

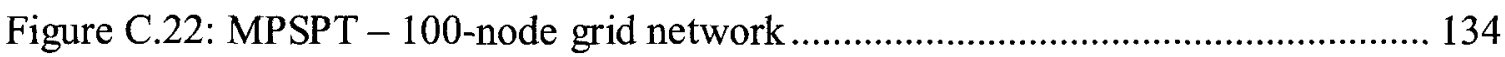

Figure C.23: MPSPT - 30-node grid network …………...................................... 134 


\section{LIST OF TABLES}

Table 2.1: IEEE 802.11 Physical Layer Standards ...................................................... 5

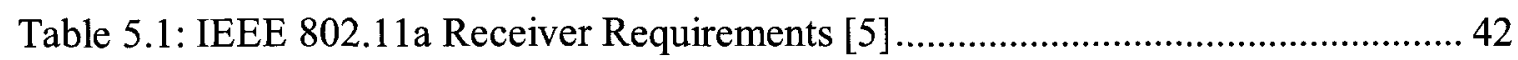

Table 5.2: IEEE 802.11 a Modulation Parameters [5] ................................................... 42

Table 5.3: Neighbor information received by Node 1 ................................................. 46

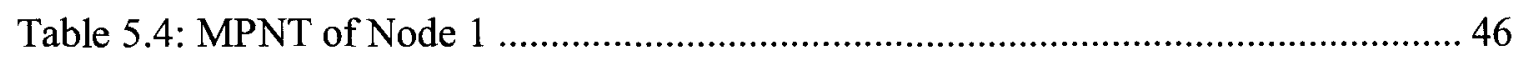

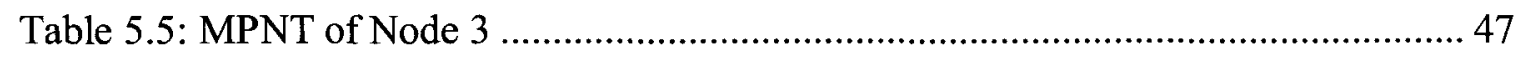

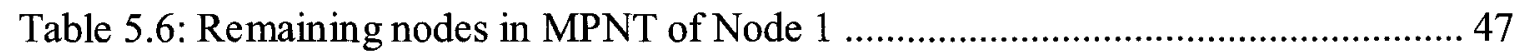

Table 5.7: DNT of Node 1 for Select 1 for less than 1 TCA ........................................... 47

Table 5.8: DNT of Node 1 for Select 2 for less than 2 TCA ............................................ 48

Table 5.9: DNT of Node 1 for Select 3 for less than 3 TCA .......................................... 48

Table 5.10: DNT of Node 7 for Select 3 for less than 3 TCA …….................................. 48

Table 5.11: FNT of Node 7 for Select 3 for less than 3 TCA ........................................... 49

Table 5.12: FNT of Node 1 for Select 3 for less than 3 TCA ......................................... 49

Table 5.13: FNT of Node 1 with power required to reach its neighbors ......................... 49

Table 5.14: Possible Links for the gateway …………….............................................. 53

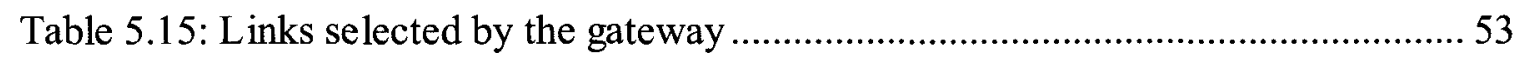

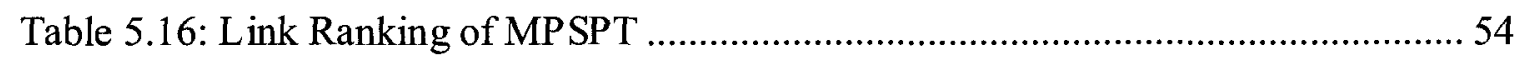

Table 5.17: Channel Assignment of the 11 highest-ranked links .................................... 55

Table 5.18: Channel Assignment of links within IR of $12^{\text {th }}$-ranked link ..........................57

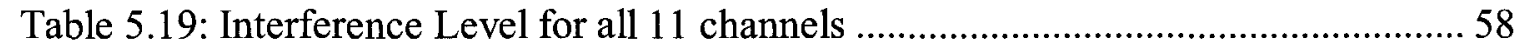

Table 5.20: Channel Assignment of links within IR of $13^{\text {th }}$-ranked link...........................59

Table 5.21: Channel Assignment for links of MPSPT ……………............................ 59

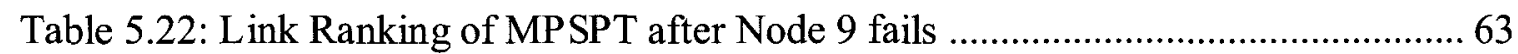

Table 5.23: Channel Assignment for links of MPSPT after Node 9 fails.......................... 64

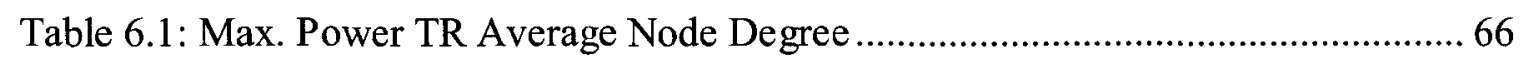

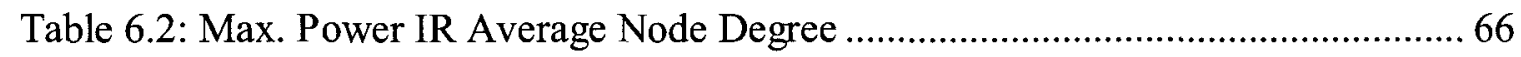

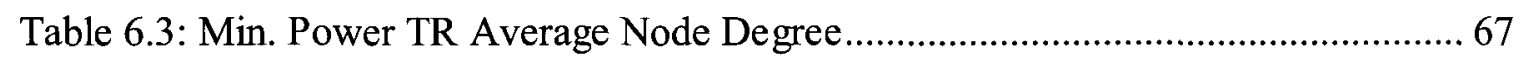

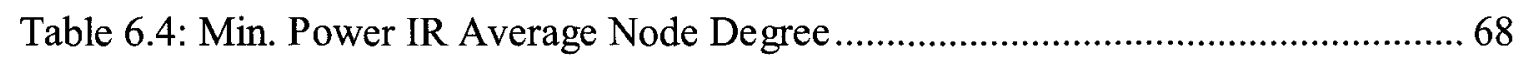

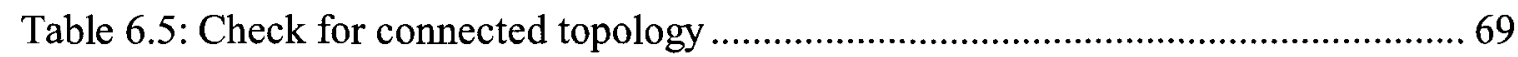

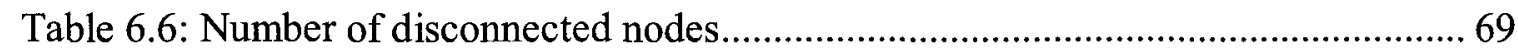

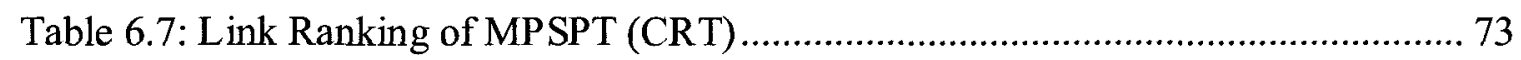

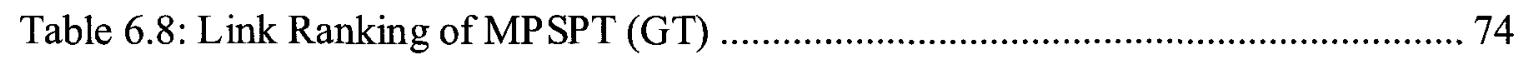

Table 6.9: Channel Assignment for links of MPSPT (CRT) ………………………...... 75

Table 6.10: Channel Assignment for links of MPSPT (GT) ....................................... 76

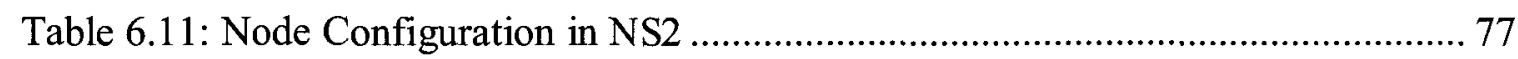

Table 6.12: Physical Layer Node Configuration in NS2 …………………………..... 78 
Table 6.13: MAC Layer Node Configuration in NS2 ............................................. 79

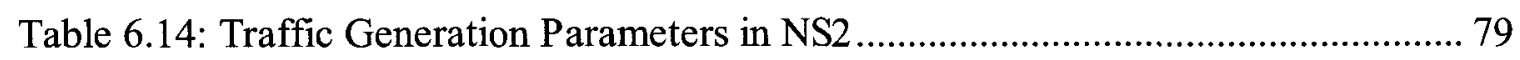

Table 6.15: AT (Mbps) for ten random topologies - 36-node network........................... 80

Table 6.16: Throughput Analysis for different RTs - 36-node network ....................... 82

Table 6.17: TIF (RT) - 36-node network ............................................................... 83

Table 6.18: AT (Mbps) for ten controlled random topologies - 36-node network ......... 84

Table 6.19: Throughput Analysis for different CRTs - 36-node network ...................... 85

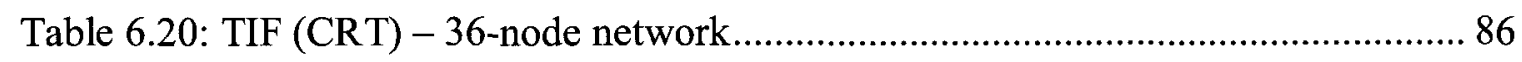

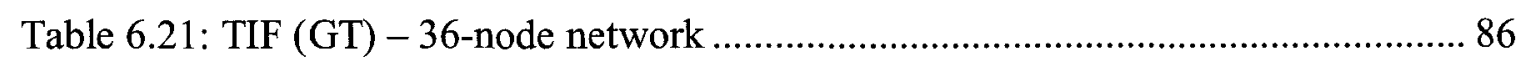

Table 6.22: Throughput Analysis for GT - 36-node network ....................................... 87

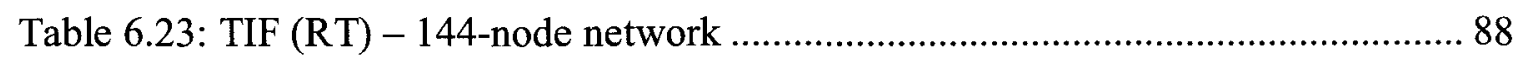

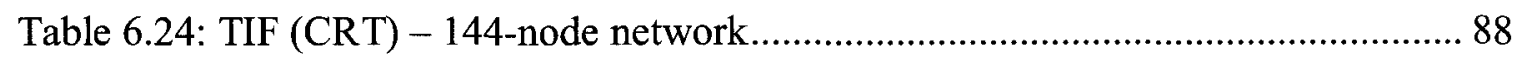

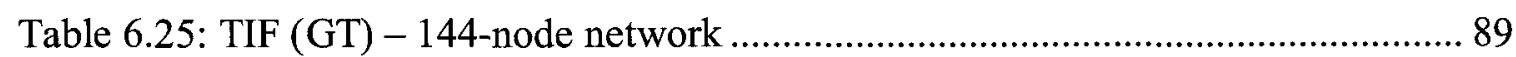

Table 6.26: Throughput Analysis - 144-node network .............................................. 90

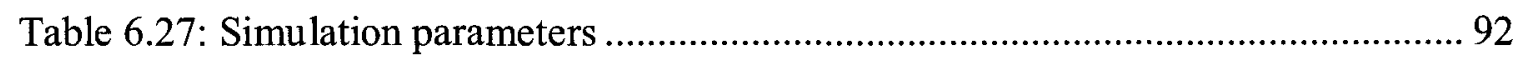

Table 6.28: AT (Mbps) with ten different TPs .......................................................... 93

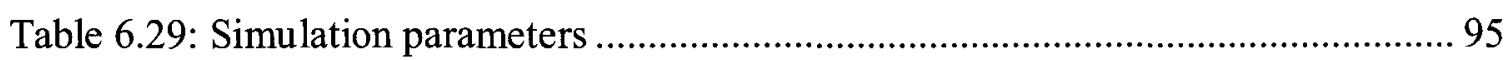

Table 6.30: AT (Mbps) with ten different TPs ..................................................... 96

Table 6.31: Features' Comparison of Related CA Schemes.......................................... 98

Table A.1: FNTs of all nodes resulting from Select 1 for less than 1 TCA ................... 108

Table A.2: FNTs of all nodes resulting from Select 2 for less than 2 TCA.................. 109

Table A.3: FNTs of all nodes resulting from Select 3 for less than 3 TCA .................. 110

Table B.1: Link Ranking of MPSPT after Node 3 fails ........................................... 113

Table B.2: Channel Assignment for links of MPSPT after Node 3 fails ....................... 114

Table B.3: Link Ranking of MPSPT after Node 30 fails .......................................... 116

Table B.4: Channel Assignment for links of MPSPT after Node 30 fails ..................... 117

Table B.5: Link Ranking of MPSPT after Node 5 fails ........................................... 119

Table B.6: Channel Assignment for links of MPSPT after Node 5 fails ....................... 120 


\section{LIST OF ACRONYMS AND ABBREVIATIONS}

ACK Acknowledgement

AND Average Node Degree

AODV Ad hoc On-Demand Distance Vector

AP Access Point

AT Average Throughput

BFS-CA Breadth First Search - Channel Assignment

CA Channel Assignment

CAA Channel Assignment Algorithm

CARM Channel Assignment and Routing Message

CBR Constant Bit Rate

CCA Common Channel Assignment

C-HYA Centralized Hyacinth

CRT Controlled Random Topology

CS Carrier Sensing

CSMA/CA Carrier Sense Multiple Access with Collision Avoidance

CTS Clear to Send

DCF Distributed Coordination Function

D-HYA Distributed Hyacinth

DNT Direct Neighbor Table

FNT Final Neighbor Table

FRM Failure Recovery Mechanism

FTP File Transfer Protocol

GT Grid Topology

GW Gateway

HMCP Hybrid Multi-Channel Protocol

IL Interference Level

IR Interference Range

ISM Industrial, Scientific, and Medical Band

LAN Local Area Network

LIC Least Interfering Channel 
MAC Medium Access Control

MANET Mobile Ad hoc Network

MCG Multi-Radio Conflict Graph

MesTiC Mesh-based Traffic and interference-aware Channel-assignment

MMAC Multi-channel Medium Access Control

MND Maximum Node Degree

MPNT Maximum Power Neighbor Table

MPSPT Minimum Power-based Shortest Path Tree

MR Mesh Router

MRMC Multi-Radio Multi-Channel

NS2 Network Simulator Version 2

PCF Point Coordination Function

PHY Physical Layer

RT Random Topology

RTS Request to Send

SPT Shortest Path Tree

SRSC Single-Radio Single-Channel

STA Station

TC Topology Control

TCA Topology Control Algorithm

TCP Transmission Control Protocol

TICA Topology-controlled Interference-aware Channel-assignment Algorithm

TIF Throughput Improvement Factor

TP Traffic Profile

TPC Transmit Power Control

TR Transmission Range

UDP User Datagram Protocol

VCA Varying Channel Assignment

WLAN Wireless Local Area Network

WMN Wireless Mesh Network

WSN Wireless Sensor Network 


\section{CHAPTER 1}

\section{INTRODUCTION}

In this chapter, an overview of Wireless Mesh Networks (WMNs) is presented, and some unique features which distinguish WMNs from Mobile Ad hoc Networks (MANETs) and Wireless Sensor Networks (WSNs) are listed. The main purpose of this thesis is discussed, and the thesis contribution is presented along with the main features of this work.

\subsection{Overview}

The use of Wireless Local Area Networks (WLANs) has grown tremendously in the past few years due to their ease of deployment and maintenance. However, the access points in these WLANs have to be connected to the backbone network through wired media. Wireless Mesh Networks offer an attractive alternative for providing broadband wireless Internet connectivity by using a wireless backhaul network and eliminating the need for extensive cabling.

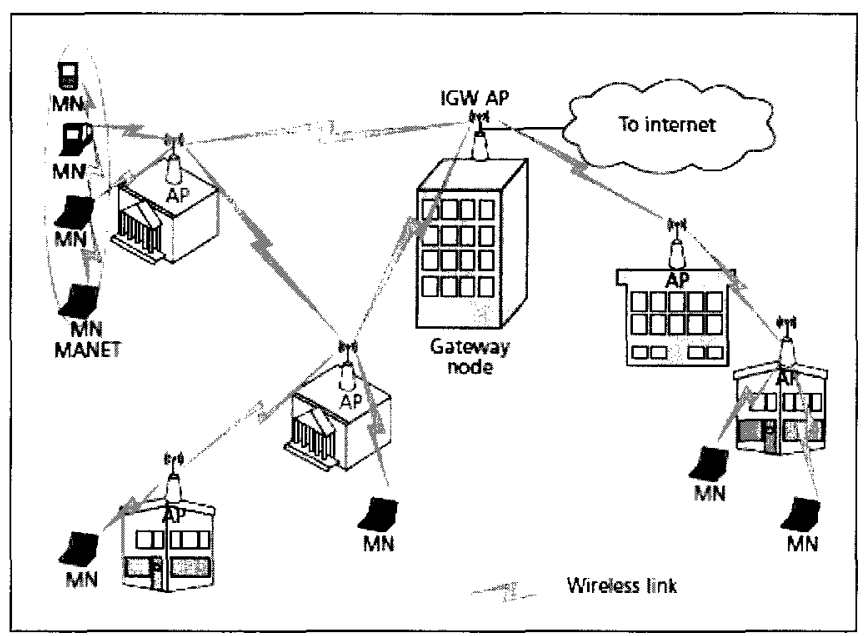

Figure 1.1: A sample enterprise WMN [1]

In traditional WLANs, each Access Point (AP) is connected to the wired network while only a subset of APs is connected to the wired network in WMNs. An AP that is connected to the wired network is called Gateway (GW); APs without wired connections are called Mesh Routers (MRs), and they connect to the GW through multiple hops. Like routers in a wired network, MRs in a WMN forward each other's traffic to establish and 
maintain their connectivity. MRs and GWs are similar in design, with the only difference that a GW is directly connected to the wired network, while a MR is not. Figure 1.1 shows a sample mesh network in a typical enterprise such as a university [1].

\section{Distinguishing WMNs from MANETs and WSNs}

The following are some unique features that distinguish WMNs from MANETs and WSNs [1]:

\section{- Mesh routers are static}

Mesh routers in a WMN are stationary; therefore, the route selection should focus on discovering links that interfere with as few nodes as possible to provide high endto-end throughput.

\section{- Mesh routers have no power constraint}

In contrast to traditional wireless networks, such as MANETs and WSNs, where nodes are typically power-constrained, MRs have abundant power at their disposal.

\section{- Mesh routers have multiple radios}

With the reduced cost of radios, MRs can be equipped with multiple radios. Hence, simultaneous transmission and reception can be achieved using intelligent channel assignment to these radios.

\section{- The traffic model is different}

In MANETs, traffic can be from any peer Mobile Node (MN) to any other MN, while in WMNs, traffic is between MRs and the GW.

\section{- Traffic is concentrated along certain paths}

In MANETs, traffic distribution is generally assumed to be uniform, while in WMN, traffic is concentrated along the paths directed towards the GW.

\section{- Traffic volume is high}

MANETs have been designed essentially for enabling communication within a small group of people, while WMNs aim to provide high-bandwidth broadband connections to a large community, and thus should be able to accommodate a large number of users accessing the Internet. Due to high estimated traffic volume in WMNs, scalability and fault tolerance become important considerations in algorithm design. 


\subsection{Motivation}

To the best of my knowledge, the proposed channel assignment algorithm is the first of its kind to use topology control based on power control for channel assignment in multi-radio multi-channel wireless mesh networks.

The main purpose of network topology control using power control is to minimize the interference between a MR and other MRs in the network by adjusting its transmission range using transmission power control. This leads to a better frequency reuse during channel assignment which results in achieving the objective of significant improvement in the overall network throughput.

\subsection{Thesis Contribution}

Specifically, the contribution of this thesis is as follows: "A new Topologycontrolled Interference-aware Channel-assignment Algorithm (TICA) which intelligently assigns the available non-overlapping 802.11 a frequency channels to the mesh routers with the objective of minimizing interference and, thereby, improving network throughput." The main features of this work are as follows:

- A Topology Control Algorithm (TCA) named Select $x$ for less than $x$; that builds the network topology by selecting the nearest neighbors for each node in the network, with the objective of minimizing interference among MRs and enhancing frequency reuse

- A scheme that uses the minimum power as the link weight when building the Shortest Path Tree (SPT) with the required node degree with the objective of minimizing interference and enhancing frequency reuse

- A Channel Assignment Algorithm (CAA), TICA, which assigns the available nonoverlapping 802.11a frequency channels to the mesh nodes with the objective of improving the overall network throughput by minimizing interference between mesh nodes as well as ensuring connectivity between them

- A concept of assigning the Least Interfering Channel (LIC) to a link if all the available frequency channels have already been assigned within the interference range of that link (LIC is the channel with the minimum interference level, which means that assigning this channel to the link would result in minimum interference in the network.) 
- A scheme that builds the Interference Level (IL) for all the frequency channels if all the available frequency channels have already been assigned within the interference range of a link

- A centralized Failure Recovery Mechanism (FRM) for TICA which provides automatic and fast failure recovery by reorganizing the network in order to bypass the failed node and to restore connectivity

\subsection{Thesis Organization}

The rest of the thesis is organized as follows. Chapter 2 gives an overview of IEEE 802.11 standard, including a brief description of its physical layer and medium access mechanism. Different IEEE 802.11 standards including $802.11 \mathrm{a}, \mathrm{b}$ and $\mathrm{g}$, are briefly discussed, along with their respective transmission technologies. The chapter also includes a brief overview of the IEEE $802.11 \mathrm{~s}$ standard for wireless mesh networks. The related work on topology control and CAAs is presented in this chapter.

Chapter 3 presents the medium access issues encountered by IEEE 802.11 singleradio single-channel nodes. It discusses the connectivity and interference trade-off, which is an important issue in WMNs. Finally, the channel assignment problem is presented with respect to multi-radio multi-channel wireless mesh network.

Chapter 4 presents the network architecture for the proposed model and the proposed topology control algorithm and CAA, along with the details of their respective phases. The FRM of the proposed CAA is also presented in this chapter.

Chapter 5 provides a performance evaluation of the proposed CAA. The network topologies used for performance evaluation are presented, and details on the working of different phases and FRM of TICA are presented with examples.

Chapter 6 contains the results for the different phases of the proposed CAA including topology control, connectivity graph, minimum power based SPT with the required maximum node degree, link ranking and channel assignment. The results of simulations for performance evaluation based on throughput analysis of a 36-node network and a 144-node network are presented in this chapter. The chapter also presents the results for the performance comparison of TICA with two well-known centralized channel assignment schemes.

Chapter 7 presents the conclusions, along with some directions for future work. 


\section{CHAPTER 2}

\section{BACKGROUND}

In this chapter, an overview of the IEEE 802.11 standard for WLANs is given by highlighting some important details of the Physical (PHY) and Medium Access Control (MAC) layers defined by this standard. Various IEEE 802.11 standards including a, b and $\mathrm{g}$, are discussed, along with IEEE 802.11s, which is being developed for multihop wireless mesh networks. The chapter discusses the effects of topology control on the operation of a network, gives the taxonomy of the topology control schemes for multihop wireless networks, and presents some related well-known topology control algorithms. The chapter also contains a taxonomical classification of the channel assignment schemes for wireless mesh networks, and discusses some related well-known channel assignment algorithms for each class.

\subsection{IEEE 802.11 Standard}

The IEEE 802.11 family of standards is currently the most successful wireless networking standards for WLANs. The initial specification was completed in 1999 (IEEE $802.11 \mathrm{a} / \mathrm{b}$ ) [2] and later extended in 2003 (IEEE 802.11g). It defines the PHY and MAC layer for the devices used in WLAN. The physical layer of the IEEE 802.11 protocol architecture describes the frequency band, data rate, and transmission technology, as shown in Table 2.1 .

Table 2.1: IEEE 802.11 Physical Layer Standards

\begin{tabular}{|c|c|c|c|c|c|c|}
\hline $\begin{array}{c}\text { IEEE } \\
\text { Standard }\end{array}$ & Spectrum & $\begin{array}{c}\text { Max. } \\
\text { Physical } \\
\text { Rate }\end{array}$ & $\begin{array}{c}\text { Transmission } \\
\text { Technology }\end{array}$ & $\begin{array}{c}\text { Compatible } \\
\text { with }\end{array}$ & $\begin{array}{c}\text { Major } \\
\text { Disadvantages }\end{array}$ & $\begin{array}{c}\text { Major } \\
\text { Advantages }\end{array}$ \\
\hline $\mathbf{8 0 2 . 1 1}$ & $2.4 \mathrm{GHz}$ & $2 \mathrm{Mbps}$ & FHSS/DSSS & None & Limited bit rate & Higher range \\
\hline $\mathbf{8 0 2 . 1 1 a}$ & $5.0 \mathrm{GHz}$ & $54 \mathrm{Mbps}$ & OFDM & None & $\begin{array}{c}\text { Smallest range of } \\
\text { all } 802.11 \\
\text { standards }\end{array}$ & $\begin{array}{c}\text { Higher bit rate in } \\
\text { less-crowded } \\
\text { spectrum }\end{array}$ \\
\hline $\mathbf{8 0 2 . 1 1 b}$ & $2.4 \mathrm{GHz}$ & $11 \mathrm{Mbps}$ & DSSS & 802.11 & $\begin{array}{c}\text { Bit rate too low } \\
\text { for many } \\
\text { emerging } \\
\text { applications }\end{array}$ & $\begin{array}{c}\text { Widely } \\
\text { deployed; } \\
\text { higher range }\end{array}$ \\
\hline $\mathbf{8 0 2 . 1 1 g}$ & $2.4 \mathrm{GHz}$ & $54 \mathrm{Mbps}$ & OFDM & $\begin{array}{c}802.11 \\
\text { and } \\
802.11 \mathrm{~b}\end{array}$ & $\begin{array}{c}\text { Limited no. of co- } \\
\text { located WLANs } \\
\text { due to narrow } \\
\text { spectrum }\end{array}$ & $\begin{array}{c}\text { Higher bit rate in } \\
\text { 2.4 GHz band; } \\
\text { higher range } \\
\text { than } 802.11 \mathrm{a}\end{array}$ \\
\hline
\end{tabular}




\subsubsection{Physical Layer Transmission Technologies}

The original IEEE 802.11 standard defines the MAC layer and three different PHY layers. The transmission technologies that the original standard defined are as follows [3]:

- Frequency-Hopping Spread Spectrum (FHSS)

- Direct-Sequence Spread Spectrum (DSSS)

- Infrared (IR)

A fourth physical layer transmission technology, Orthogonal Frequency Division Multiplexing (OFDM), was introduced later.

DSSS operates in the $2.4 \mathrm{GHz}$ ISM band, at data rates of $1 \mathrm{Mbps}$ and $2 \mathrm{Mbps}$. The available number of channels ranges from 13 in most countries to just one in Japan. The DSSS PHY operates in the frequency range of $2.4 \mathrm{GHz}$ to $2.4835 \mathrm{GHz}$ in North America and $2.471 \mathrm{GHz}$ to $2.497 \mathrm{GHz}$ in Japan. Out of 13 available channels in North America, only channels 1, 6 and 11 are not overlapping, as shown in Figure 2.1 [4].

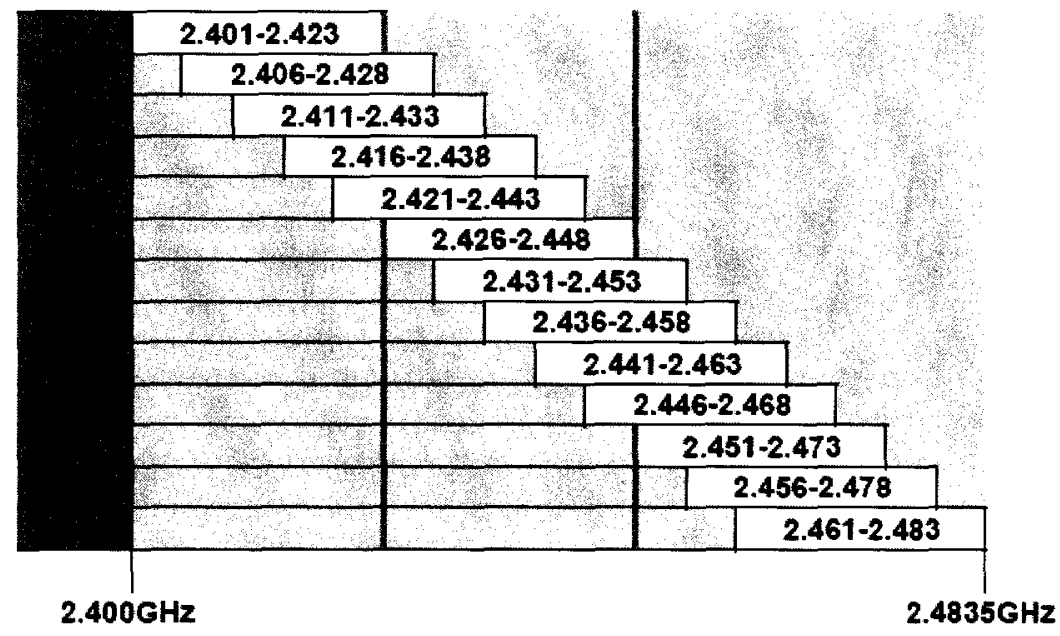

\section{Figure 2.1: Available channels for DSSS in the 2.4 GHz ISM band [4]}

OFDM, also called multicarrier modulation, uses multiple carrier signals at different frequencies, sending bits on each carrier. This is similar to FDM (Frequency Division Multiplexing), which uses each subchannel for a separate data source. OFDM, however, dedicates all of the subchannels to a single data source. The possible data rates per channel range from 6 to $54 \mathrm{Mbps}$. The support of transmitting and receiving at data rates of 6,12 , and $24 \mathrm{Mbps}$ is mandatory for $20 \mathrm{MHz}$ channel spacing. The system uses 52 subcarriers, of which only 12 are non-overlapping [5]. 


\subsubsection{Medium Access Control Layer}

The IEEE 802.11 MAC layer has two main sublayers. The lower one, Distributed Coordination Function (DCF), uses an Ethernet-style contention algorithm that provides access to all traffic. The upper MAC sublayer, Point Coordination Function (PCF), is a centralized MAC algorithm that provides contention-free service by polling STAs in turn $[3]$.

DCF is the basic medium access protocol of the IEEE 802.11 MAC that allows distributed medium sharing between compatible physical layers through the use of Carrier Sense Multiple Access with Collision Avoidance (CSMA/CA) and a random backoff time following a busy medium condition [5].

Carrier Sensing (CS) is performed both through physical and virtual mechanisms. A physical CS mechanism is provided by the physical layer, while a virtual CS mechanism is provided by the MAC layer.

\section{A. Physical CS using CSMA/CA}

In physical CS, a STA senses the medium before it transmits, in order to determine if another STA is transmitting. If the medium is not busy, the transmission may proceed. The CSMA/CA-distributed algorithm requires that a gap of a minimum

specified duration exist between frame sequences. A transmitting STA ensures that the medium is idle for this required duration before attempting to transmit. If the medium is determined to be busy, the STA defers until the end of the current transmission. After deferral, or prior to attempting to transmit again immediately after a successful transmission, the STA selects a random backoff interval and decrements the backoff interval counter while the medium is idle. A transmission is successful when an ACK frame is received from the addressed STA [5].

\section{B. Virtual CS using Request to Send and Clear to Send}

The virtual CS mechanism is achieved by distributing reservation information announcing the impending use of the medium. The exchange of Request to Send (RTS) and Clear to Send (CTS) frames prior to the actual data frame is used for the distribution of the medium reservation information. The RTS and CTS frames contain a duration field that defines the period of time the medium is to be reserved in order to transmit the actual 
data frame and the returning ACK frame. All STAs within the reception range of either the originating STA (which transmits the RTS) or the destination STA (which transmits the CTS) can learn of the medium reservation [5].

\subsubsection{IEEE 802.11a}

The IEEE 802.11a specification uses the 5-GHz band and was established so that the users could take advantage of a newly allocated unlicensed radio band, the Unlicensed National Information Infrastructure (UNII) band. The FCC (Federal Communications Commission, a United States government agency) created UNII to support the development of high-speed wireless networks. To meet the increasing bandwidth demand, FCC established the band at $5 \mathrm{GHz}$, making it incompatible with 2.4$\mathrm{GHz}$ equipment.

\subsubsection{IEEE 802.11b}

IEEE $802.11 \mathrm{~b}$ is an extension of the IEEE 802.11 DSSS scheme, providing data rates of up to $11 \mathrm{Mbps}$. Like an IEEE 802.11 DSSS channel, each IEEE 802.11b channel requires the same bandwidth. To achieve a higher data rate in the same bandwidth, the standard employs a modulation scheme called Complementary Code Keying (CCK) [5]. IEEE $802.11 \mathrm{~b}$ is currently the most commonly used IEEE 802.11 standard in commercial products.

\subsubsection{IEEE 802.11g}

IEEE 802.11g extends IEEE 802.11b to data rates of up to $54 \mathrm{Mbps}$ per channel. IEEE $802.11 \mathrm{~g}$ is compatible with IEEE $802.1 \mathrm{lb}$ because they both operate in the $2.4 \mathrm{GHz}$ range. The key difference between IEEE 802.11b and IEEE 802.11g is that the latter uses OFDM and DSSS rather than DSSS only. With this standard, IEEE 802.11b devices will work when connected to an IEEE $802.11 \mathrm{~g}$ access point and vice versa, in both cases using the lower IEEE $802.11 \mathrm{~b}$ data rate.

\subsection{IEEE 802.11s for Wireless Mesh Networks}

The IEEE 802.11s [6] mesh networking standard aims at applying multihop routing techniques to extend the range of WLAN. Hence, the WLAN mesh can be 
considered as a set of access points interconnected with each other via wireless links, resulting in a "mesh of connectivity."

The IEEE 802.11s Task Group, formed in 2004, is in the process of developing specifications of a new protocol suite for the installation, configuration, and operation of WLAN mesh. It will be implemented on top of the existing PHY layer of IEEE $802.11 \mathrm{a} / \mathrm{b} / \mathrm{g}$ operating in the unlicensed spectrum of 2.4 and $5 \mathrm{GHz}$ frequency bands [7].

\subsubsection{Network Architecture}

In IEEE $802.11 \mathrm{~s}$, a meshed wireless LAN is formed where access points do not need to be connected by wired LANs and are connected by wireless mesh networking, with multiple hops in between. Portals, which are also called Gateways (GWs), are used to connect access points with the Internet. Typical network architecture of IEEE 802.11s is shown in Figure 2.2 [8].

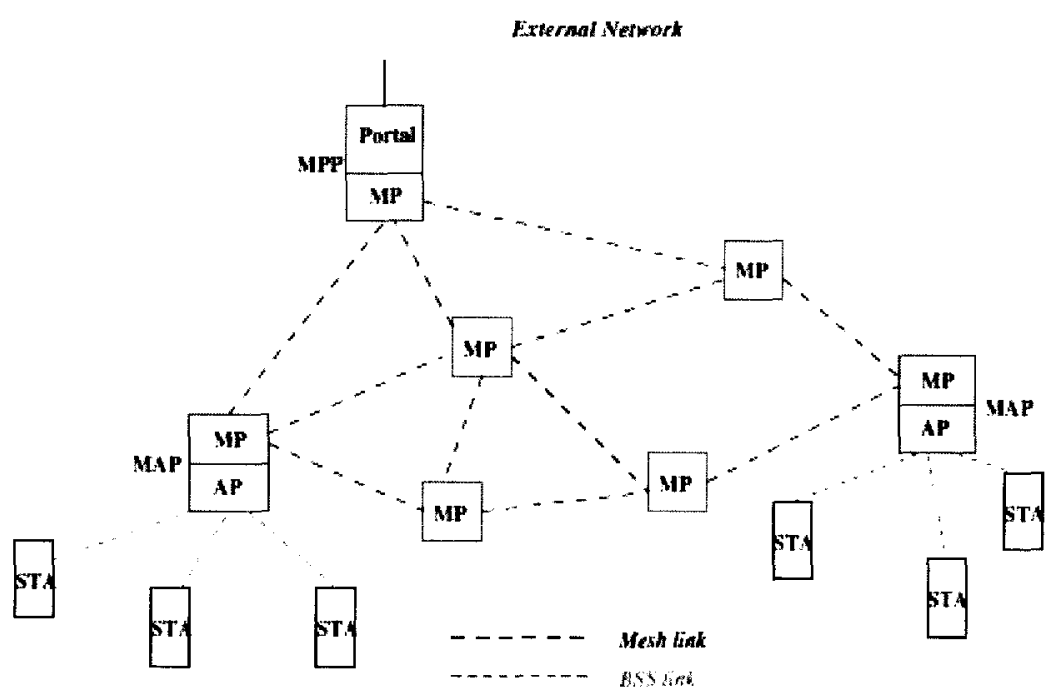

Figure 2.2: IEEE 802.11s Mesh Network Architecture [8]

There are three types of nodes in this architecture.

\section{A. Mesh Point (MP)}

Any node that supports the mesh services, including neighbor discovery, channel selection, and forming an association with neighbors, is a Mesh Point [9]. MPs can directly communicate with their neighbors and forward traffic on behalf of other MPs.

\section{B. Mesh Access Point (MAP)}

In addition to being a MP, if a mesh node acts as an access point by supporting access to client stations; it is called a Mesh Access Point (MAP). 


\section{Mesh Portal (MPP)}

A Mesh Portal (MPP) is a MP that has a non-802.11 wired connection to the Internet and serves as a logical point for MAC Service Data Units (MSDUs) to enter or exit the mesh network. A MPP and a MAP may be co-located on a single device.

\subsubsection{Protocol Stack}

The protocol stacks of the three types of nodes described above are illustrated in Figure $2.3[8]$.

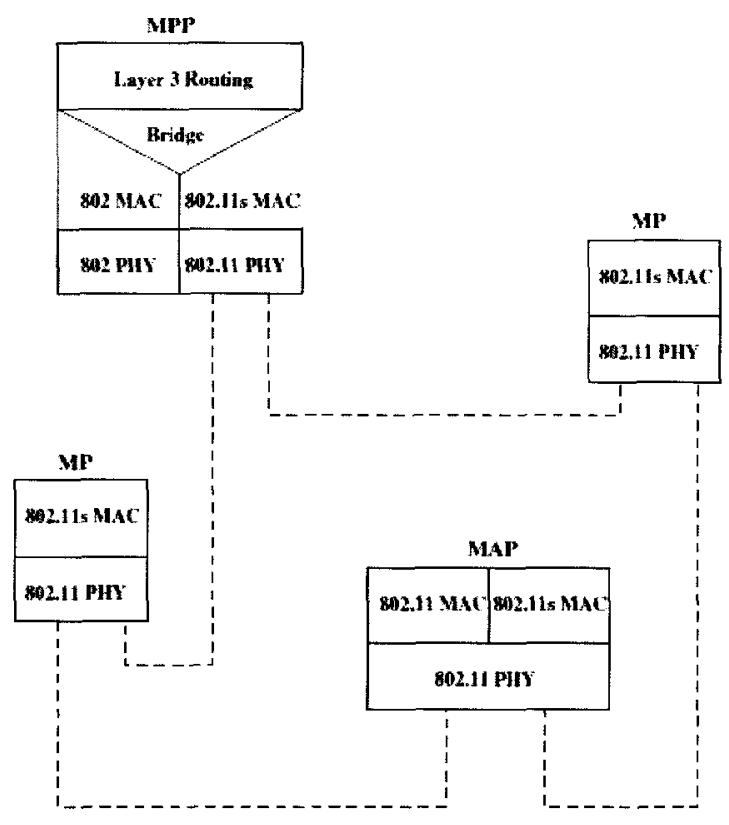

Figure 2.3: IEEE 802.11s Protocol Stack [8]

The IEEE 802.11s MAC is based on the existing IEEE 802.11 MAC, and the mesh routing protocol resides in the MAC layer. In a MPP, a layer-3 routing protocol is also needed for path selection from the mesh network to external network or vice versa [8].

\subsection{Topology Control Schemes}

The importance of Topology Control (TC) lies in the fact that it affects network spatial reuse and hence the traffic carrying capacity. Choosing a large transmit power results in excessive interference, while choosing a small transmit power results in a disconnected network [10]. Using TC through transmission power control, the network connectivity and hence the network topology is affected, interference levels are 
mitigated, which reduces the co-channel interference, and the opportunity of spatial channel re-use is enhanced [11].

\subsubsection{Topology Control in Multi-radio WMNs}

One method for reducing the co-channel interference is to appropriately select the channels for the wireless interfaces. The channel assignment problem in multi-radio wireless mesh networks involves assigning a channel to each wireless interface. In addition to reducing the level of interference, channel assignment also affects the connectivity of wireless mesh networks, since two interfaces within the transmission range of each other can communicate only if they operate on the same channel. There is a trade-off between maximizing the connectivity and reducing the level of interference.

The connectivity graph in a multi-radio WMN is determined through topology control. So, the problem of TC in multi-radio WMNs involves the selection of transmission power for each radio interface of each mesh node in the network, so as to maintain the network connectivity with the use of minimum power.

\subsubsection{Effect of Topology Control}

The problem of TC is complex, since the choice of the transmit power fundamentally affects many aspects of the operation of the network [12].

- The transmit power level determines the quality of the signal received at the receiver.

- It determines the transmission range.

- It determines the interference range, i.e. the magnitude of the interference created for the other receivers.

\section{A. Effect of TC on the OSI layers}

Due to the following factors, Topology Control (TC) via Transmit Power Control (TPC) affects the following layers of the OSI protocol stack:

- It affects the physical layer as the transmit power level affects the Received Signal Strength (RSS).

- It affects the MAC layer because the interference range affects the spatial channel reuse. 
- It affects the network layer because the transmission range affects routing.

- It affects the transport layer because interference causes congestion.

\section{B. Effect of TC on the Performance of the Network}

TC via TPC has a multi-dimensional effect on the performance of the whole network:

- The transmit power levels determine the performance of medium access control, since the spatial channel reuse depends on the number of other nodes within the interference range.

- The choices of power levels affect the connectivity of the network, and consequently the ability to deliver a packet to its destination [13].

- The power level affects the throughput capacity of the network [14].

- Power control affects the network topology which affects the number of hops, and thus the end-to-end delay.

\section{Effect of TC on the Performance of the MAC and Routing Protocols}

In addition, the assumption of fixed power levels is so ingrained into the design of many protocols in the OSI stack that changing the power levels results in their malfunctioning.

- Changing power levels can create uni-directional links, which can happen when a node $i$ 's power level is high enough for a node $j$ to hear it, but not vice versa.

- Bi-directionality of links is implicitly assumed in many routing protocols.

- Medium access control protocols in IEEE 802.11 implicitly rely on bidirectionality assumption of links.

\subsubsection{Guidelines for an Effective Topology Control Algorithm}

The following guidelines are essential to an effective Topology Control Algorithm (TCA) [10]:

- The network connectivity should be preserved with the use of minimum possible power. This is the most important objective of topology control algorithms, as less transmit power translates to less interference.

- The algorithm should be distributed and should depend only on the information collected locally, e.g. information collected within one hop. Algorithms that 
depend only on local information incur less message-overhead in the process of collecting information.

- It is desirable that all links be bi-directional. As mentioned earlier, bi-directional links are required to facilitate link level acknowledgment and ensure the existence of reverse paths.

- It is also desirable that the node degree in the topology derived under the algorithm is small. A small node degree helps to mitigate co-channel interference and enhance spatial channel reuse.

\subsubsection{Taxonomy of Topology Control Schemes}

These schemes are mainly divided into two types [15].

\section{A. Homogeneous}

This is the basic type of TC, as all the nodes are assumed to use the same transmitting range. So, the topology control problem reduces to determining the minimum value of transmission range that ensures network connectivity. This minimum transmission range is also called the Critical Transmitting Range (CTR).

\section{B. Non-homogeneous}

In this type of $\mathrm{TC}$, nodes are allowed to choose different transmitting ranges, provided they do not exceed the maximum range. Depending on the type of information that is used to compute the topology, non-homogeneous topology control is further classified into three categories.

\section{i) Location-based schemes}

In such schemes, exact node positions are known. If this information is used by a centralized authority to compute a set of transmitting range assignments which optimizes a certain measure such as the energy cost, this is the case of the Range Assignment Problem and its variants. If, however, this information is exchanged between nodes and used to compute an "almost optimal" topology in a fully distributed manner, this is the case for protocols used for building energy-efficient topologies for unicast or broadcast communication. 


\section{ii) Direction-based schemes}

In such schemes, it is assumed that nodes do not know their position, but they can estimate the relative direction of each of their neighbors.

\section{iii) Neighbor-based schemes}

In such schemes, nodes are assumed to know only the ID of the neighbors, and are able to order them according to some criterion such as link quality.

\subsubsection{Related Topology Control Algorithms}

The goal of the TCA for multi-radio WMNs, Select $x$ for less than $x$, proposed in this thesis, is to minimize the interference among the MRs. To achieve this goal, the proposed TCA controls the network topology by selecting the nearest neighbors for each node in the network. Some related TCAs are discussed below.

\section{A. Local Minimum Spanning Tree Algorithm}

In this algorithm, each node builds its local minimum spanning tree independently and only keeps as its neighbors those on-tree nodes that are one hop away in the final topology. It is a location-based scheme as it assumes that each node is equipped with the ability to gather its location information. Some important properties of the Local Minimum Spanning Tree (LMST) algorithm [10] include:

- The topology constructed under LMST preserves the network connectivity

- The degree of any node in the resulting topology is bounded by 6

- The resulting topology can be converted into one with bi-directional links after the removal of uni-directional links.

The feature of small node degree is desirable as it reduces interference. LMST is composed of the following three phases: information exchange, topology construction, and determination of transmission power. The phase of topology construction with bidirectional links is an optional phase.

\section{B. Enhanced Local Minimum Shortest Path Tree Algorithm}

The objective of this topology control algorithm is to find a topology that preserves certain network requirements, namely, decrease in average node degree, an averagely low power consumption which results in a longer network lifetime, and a 
maintenance of connectivity in the resultant network topology. The Enhanced Local Minimum Shortest-Path Tree (ELMST) algorithm [11] is a location-based topology control scheme and consists of two main phases:

- Construction of Local Minimum Shortest Path Tree (LMSPT)

- Removal of Unidirectional links

Each node gathers its neighbor information and constructs LMSPT, as shown in Figure 2.4.

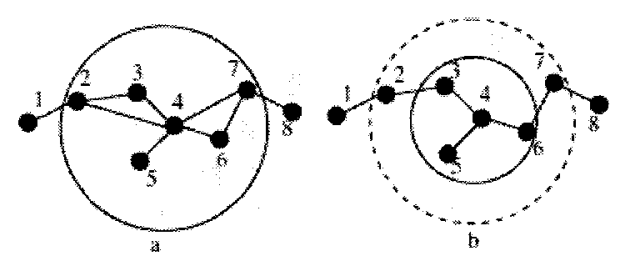

Figure 2.4: ELMST (a) before topology control (b) after topology control [11]

\section{Maximum Minimum Power Algorithm}

A drawback of WMNs is that multihop communication consumes a multiple of radio resources required to transmit a frame, thereby reducing capacity. As the wireless channel is shared by the ongoing transmissions at various hops, mutual interference either requires sequential transmissions at high data rate or spatial reuse with simultaneous transmissions at low data rates by using a robust Modulation and Coding Scheme (MCS) [14]. The objective of the Maximum Minimum Power (MMP) algorithm [16] is to maintain the best possible MCS for each link while decreasing the transmission power as much as possible.

\section{Local Mean Algorithm}

To model the behavior of the Local Mean Algorithm (LMA) [17] in WMNs, the following procedure is used in [16].

All nodes start with same initial transmission power. Every node periodically broadcasts a "Life"-Message. Upon the reception of a "Life"-Message, each node responds with a "Live-Acknowledgement"-Message. The number of responses is counted and if it is greater than a pre-set threshold "NodeMaxThres," the node decreases transmission power and repeats the algorithm. However, if the number of responses is less than or equal to the pre-set threshold "NodeMaxThres," the node does not change the transmission power and repeats the algorithm after some delay. LMA is a typical power 
control algorithm, putting emphasis on the power conservation while keeping the connectivity of the network. To ensure connectivity of the WMN, "NodeMaxThres" is set to either 4 or 5 for all nodes.

\subsection{Channel Assignment Schemes}

Channel Assignment (CA) in a multi-radio WMN environment consists of assigning channels to the radios in order to achieve efficient channel utilization (i.e. minimize co-channel interference) and, simultaneously, to guarantee an adequate level of connectivity. The problem of optimally assigning channels in an arbitrary mesh topology has been proven to be NP-hard, based on its mapping to a graph-coloring problem [18]. Therefore, channel assignment schemes employ heuristic techniques to assign channels to radios belonging to mesh nodes. A taxonomical classification of various $\mathrm{CA}$ schemes for wireless mesh networks is as follows [19].

\subsubsection{Fixed Channel Assignment Schemes}

Fixed assignment schemes assign channels to radios either permanently, or for intervals that are long with respect to the radio switching time. Such schemes can be further subdivided into two types.

\section{A. Common Channel Assignment (CCA)}

In CCA [20], the radios of each node are all assigned the same set of channels. For example, if each node has two radios, then the same two channels are used at every node. The main benefit is that the connectivity of the network is the same as that of a single channel approach, while the use of multiple channels increases network throughput. However, it does not take into account the effect of interference on the channel assignment in a WMN.

\section{B. Varying Channel Assignment (VCA)}

In the VCA scheme, the radios of different nodes are assigned different sets of channels. However, the assignment of channels may lead to network partitions and/or topology changes, which may increase the length of routes between mesh nodes. Therefore, in such a scheme, channel assignment needs to be carried out carefully. The VCA approach is discussed in more detail by presenting algorithms that belong to this category. 


\section{i) Centralized Hyacinth (C-HYA)}

C-HYA, a centralized channel assignment algorithm for multi-radio multi-channel WMNs, was proposed in [18]. Assuming that the offered traffic load is known, this algorithm assigns channels ensuring network connectivity and satisfying the bandwidth limitations of each link. It first estimates the total expected load on each virtual link by summing the load due to each offered traffic flow. Then, the channel assignment algorithm visits each virtual link in decreasing order of expected traffic load and assigns it a channel.

\section{ii) Mesh-based Traffic and interference-aware Channel-assignment (MesTiC)}

MesTiC, a fixed algorithm for centralized CA, was proposed in [21], and visits nodes once in the decreasing order of their rank. The rank of each node is computed on the basis of its link-traffic characteristics, topological properties and number of radios on a node.

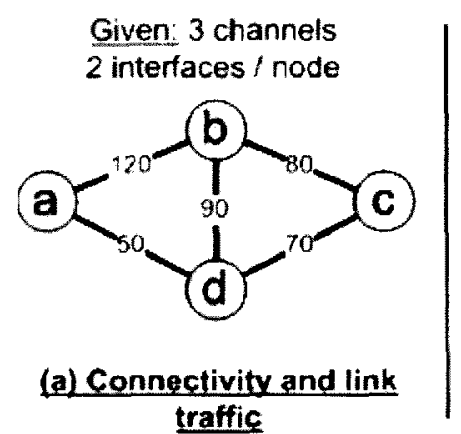

traffic

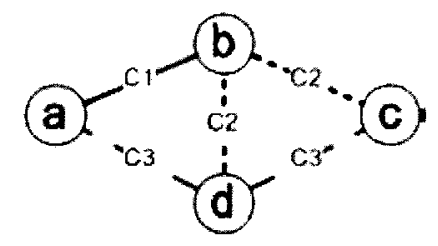

(b) Channel assianment with MesTiC

Figure 2.5: An example of MesTiC [21]

The working principle of MesTiC is illustrated in Figure 2.5 where the input connectivity graph and estimated link traffic are shown in Figure 2.5a. The network is configured with three channels and two radios per node. Assuming that node $b$ is the gateway node, the rank of the remaining nodes, in decreasing order, is $\mathrm{d}$, a, and c. The algorithm starts by visiting node $b$ first, assigning channel $\mathrm{C} 1$ to the link between $(b, a)$ (which carries the highest traffic of 120), and then moves on to assign channel C2 to the link $(b, d)$. Now, when assigning a channel to link $(b, c)$, the algorithm has to choose between $\mathrm{C} 1$ and $\mathrm{C} 2$. However, as $\mathrm{C} 1$ carries more traffic than $\mathrm{C} 2$, it assigns $\mathrm{C} 2$ to link (b, c). Likewise, at node $\mathrm{d}$, it assigns a previously unassigned channel $\mathrm{C} 3$ to the link $(\mathrm{d}, \mathrm{c})$ 
and, as $\mathrm{C} 3$ carries less traffic than $\mathrm{C} 2(90+80=170)$ or $\mathrm{C} 1(120)$, it assigns $\mathrm{C} 3$ to the link (d, a). The algorithm proceeds until all links and radios have been assigned channels, as shown in Figure 2.5b.

\subsubsection{Dynamic Channel Assignment Schemes}

In dynamic CA schemes, any radio can be assigned any channel but additionally, radios can frequently switch from one channel to another. Therefore, when nodes need to communicate with each other in such a scheme, a coordination mechanism is required to ensure that they are on a common channel.

The key challenge with the dynamic switching approach is how to coordinate decisions in terms of when to switch radios, as well as what channel to switch radios to.

\section{A. Multi-channel Medium Access Control (MMAC)}

MMAC [22] [23] is a link-layer multi-channel protocol for nodes with a single network interface. A node equipped with a single interface can only listen to one channel at a time. Therefore, in order to use multiple channels, the interface has to be switched between channels.

As mentioned above, when nodes are allowed to switch channels, a pair of nodes need to listen on the same channel at the time of communication and a channel coordination method is necessary which is not required in TICA.

\section{B. Distributed Hyacinth (D-HYA)}

D-HYA, a dynamic and distributed channel assignment algorithm proposed in [24], can adapt to traffic load dynamically. The algorithm builds on a spanning tree network topology. The gateway node is the root of a spanning tree, and every mesh node belongs to that tree. The channel assignment problem consists of the following two steps.

1. Neighbor-to-interface binding determines the interface that a node uses to communicate with each of its neighbors with whom it intends to establish a link

2. Interface-to-channel binding determines which radio channel a network interface should use.

Based on per-channel total load information, a WMN node determines the set of channels that are least used in its vicinity. As nodes higher up in the spanning trees need 
more relay bandwidth, they are given a higher priority in channel assignment. More specifically, the priority of a WMN node is equal to its hop distance from the gateway.

The CA schemes, such as C-HYA, MesTiC and D-HYA, require the traffic load to be known before assigning channels, whereas TICA requires no such knowledge for channel assignment.

\subsubsection{Hybrid Channel Assignment Schemes}

Hybrid channel assignment schemes combine both static and dynamic assignment properties by applying a fixed assignment for some radios and a dynamic assignment for other radios. The fixed radios can be assigned dedicated channels while the other radios can be switched dynamically among channels.

\section{A. Hybrid Multi-Channel Protocol (HMCP)}

HMCP [25] [26] is a link-layer multi-channel protocol for nodes with multiple radio interfaces. Out of the available interfaces at each node, $\mathrm{X}$ interfaces are assigned statically to X channels, and these interfaces are designated as "fixed interfaces." The fixed interfaces stay on the specified channels for long durations of time. The remaining interfaces can frequently switch between any of the remaining channels, based on the data traffic, and are designated as "switchable interfaces." Different nodes assign their "fixed interfaces" to different channels, thereby ensuring that all available channels are occupied, while switching the remaining interfaces (based on traffic requirements) ensures that communication between any pair of nodes is possible.

However, a co-ordination protocol is required to decide what channel to assign to the fixed interface, and also for enabling neighbors of a node $X$ to know about the channels used by fixed interface of node X. Time synchronization and coordination between mesh nodes which is required in HMCP is not needed in TICA.

\section{B. Breadth First Search - Channel Assignment (BFS-CA)}

BFS-CA [27] is a centralized, interference-aware algorithm aimed at improving the capacity of the WMN backbone and at minimizing interference. This algorithm is based on an extension to the conflict graph concept called the Multi-radio Conflict Graph (MCG) where the vertices in the MCG represent edges between radios instead of edges between mesh routers. 
To compensate for the drawbacks of a dynamic network topology, the proposed solution assigns one radio on each node to operate on a default common channel throughout the network. This strategy ensures a common network connectivity graph, provides alternate fallback routes and avoids flow distuption by traffic redirection over a default channel.

This scheme computes interference and bandwidth estimates based on the number of interfering radios, where an interfering radio is a simultaneously operating radio that is visible to a mesh router, but is external to its network. Each mesh router then derives two separate channel rankings. The first ranking depends on the increasing number of interfering radios. The second depends on the increasing channel utilization. The mesh router then merges the two rankings by taking the average of the individual ranks. The resulting ranking is used by the CA scheme. This scheme, called the Breadth First Search Channel Assignment (BFS-CA) algorithm, uses a breadth first search to assign channels to the radios. The search begins with links emanating from the gateway node, while links fanning outwards towards the edge of the network are given lower priority. To adapt to the changing interference characteristics, the algorithm periodically re-assigns channels.

BFS-CA requires certain number of MRs with certain number of radio interfaces to be placed at certain hops from the gateway, whereas TICA simply requires all MRs to have four data radios, does not require any careful router placement strategy, and works with any placement of routers as verified by a comprehensive performance evaluation.

The most significant difference between TICA and existing CA schemes is that TICA uses topology control based on power control to build the topology for CA with the objective of minimizing the interference between the MRs, whereas no other CA scheme has used topology control based on power control for CA. 


\section{CHAPTER 3}

\section{CHANNEL ASSIGNMENT PROBLEM}

In this chapter, the medium access issues encountered by IEEE 802.11-based Single-Radio Single-Channel (SRSC) WMNs are presented, and a multiple-channel approach using multiple radios to overcome these problems is discussed. The connectivity and interference trade-off in a multi-radio WMN scenario are given, and the key issue of channel assignment in multi-radio multi-channel WMNs is presented, along with its objectives and constraints.

\subsection{IEEE 802.11 Medium Access Issues}

Since the WMN has to provide access to broadband Internet, it is expected to have higher bandwidth. Even though the physical layer can support very high bit rate, current MAC protocols are not able to utilize the entire bandwidth provided by the physical layer. The main reason for this poor performance is the suboptimal media access protocols, which were primarily designed for single-hop networks [1].

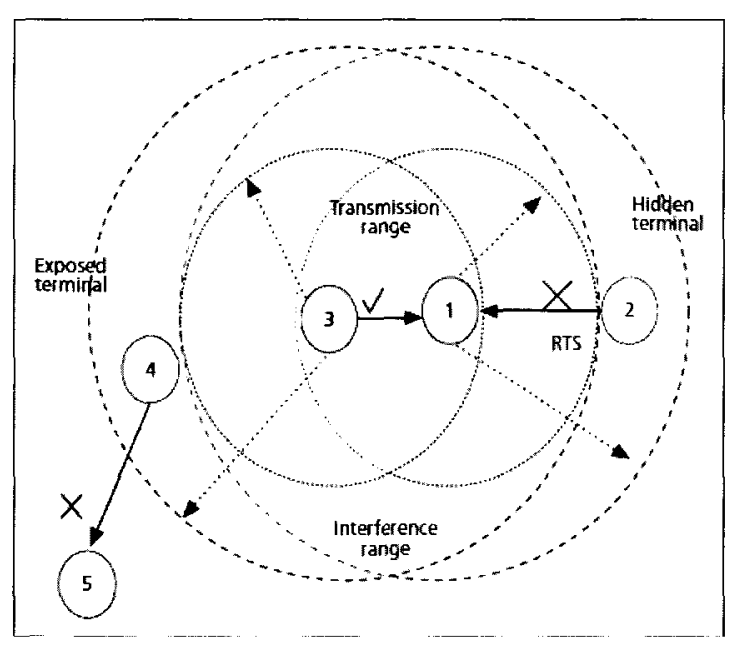

Figure 3.1: Hidden and exposed terminal problems [1]

\subsubsection{Hidden and Exposed Terminal Problems}

IEEE 802.11 DCF is one such widely accepted MAC protocol but, when used in a multihop network scenario, it results in poor performance and is therefore unacceptable. 
The reason is that some nodes remain starved due to hidden and exposed terminals in a multihop environment. Figure 3.1 illustrates these problems [1].

Node 2, which is outside the interference range of Node 3 and unaware of the ongoing transmission at Node 3 , continues to send RTS to Node 1 causing collision. This is a case of the hidden terminal problem.

Node 4 is prevented from transmitting because of the neighboring transmission at Node 3 . This is a case of the exposed terminal problem.

\subsubsection{Single-radio Single-channel Mesh Network}

A Single-Radio Single-Channel (SRSC) mesh architecture, shown in Figure 3.2a [1], suffers from hidden and exposed terminal problems. Assigning orthogonal channels to the MRs within the interference range can help alleviate the hidden and exposed terminal problems, and assist in improving the overall capacity of the network. However, considering the traffic characteristics in a WMN, frequent channel switching may be required to communicate with neighboring nodes. In such scenarios, single-radio multichannel MAC may not provide any significant performance gains due to high channel switching delay.

\subsubsection{Dual-radio Single-channel Mesh Network}

The decreasing cost of wireless radios has opened up new avenues for designing MRs using multiple radios. MRs can now use multiple radios tuned to orthogonal channels for simultaneously communicating with their neighbors. Figure $3.2 b$ shows the use of a dual-radio WMN [1]. One radio provides service to its mesh clients (using channel 6 in $2.4 \mathrm{GHz} 802.11 \mathrm{~b}$ ), and the other radio forms the backhaul link connecting all the MRs to the gateway (using channel 1 in $5 \mathrm{GHz} 802.11 \mathrm{a}$ ). However, it suffers from the disadvantage of sharing the channel with the neighboring MRs at each hop along the backhaul path to the gateway.

\subsubsection{Multi-radio Multi-channel Mesh Network}

Figure 3.2c shows the use of a multi-channel approach using multiple radios that successfully overcomes all the problems encountered in the previous architectures [1]. Two radios are employed for the backhaul link and one for servicing the mesh clients. 
The uplink and downlink backhaul radios and the service radio operate at nonoverlapping channels which eliminates the co-channel interference.

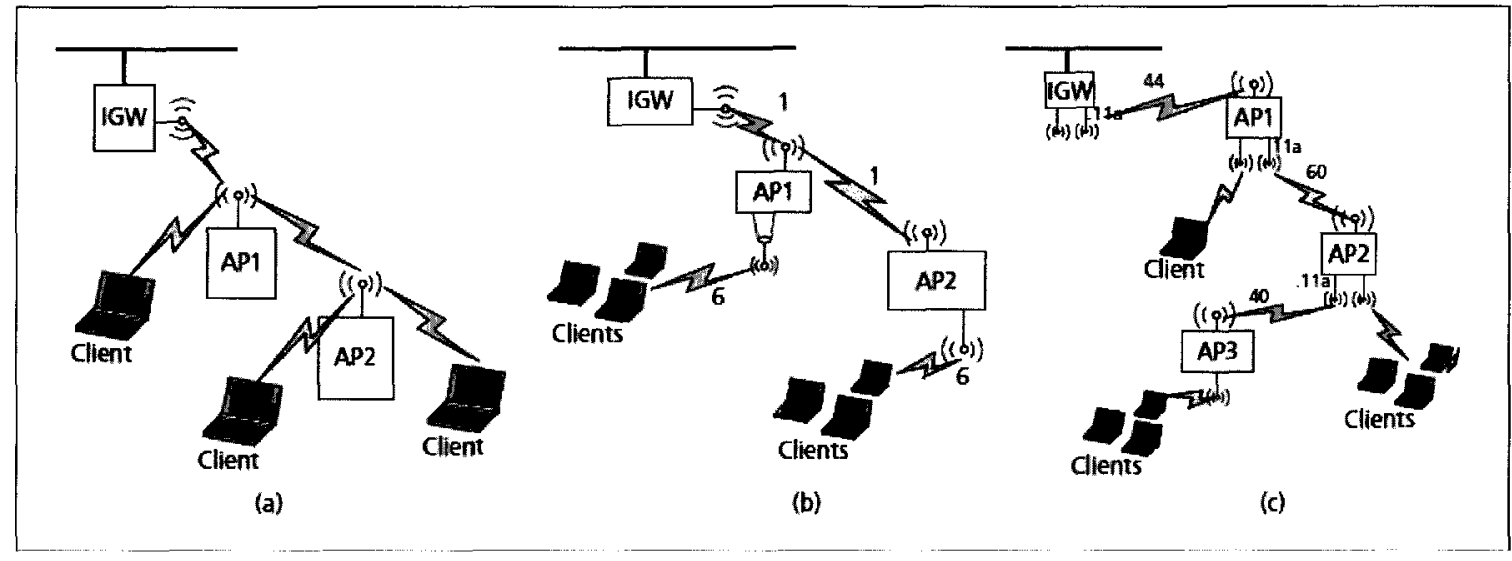

Figure 3.2: Different mesh network architectures [1]

As each mesh router can be equipped with multiple radios, fixed channel assignment to these radios is a more viable solution. Efficient and intelligent channel assignment schemes have to be designed, as the number of channels is limited.

\subsection{Connectivity and Interference Trade-off}

Equipping each node with multiple radios is emerging as a promising approach for improving the capacity of WMNs. The IEEE 802.11a standard provides 12 nonoverlapping frequency channels, which can be used simultaneously within the interference range neighborhood by assigning non-overlapping channels to radios. This leads to efficient spectrum utilization and increases the actual bandwidth available to the network [21].

Although multi-radio mesh nodes have the potential to significantly improve the performance of mesh networks, efficient channel assignment is a key issue in guaranteeing network connectivity while still mitigating the adverse effects of interference from the limited number of channels available to the network. A WMN node needs to share a common channel with each of its communication-range neighbors with which it needs to set up a link. However, to reduce interference, a node should minimize the number of neighbors with which it shares a common channel. Therefore, there is a trade-off between maximizing connectivity and minimizing interference, as illustrated in Figure 3.3 [19]. 


\subsubsection{Maximum Connectivity in a Single-radio Single-channel Scenario}

Fig. 3.3a shows the connectivity of the network when a single radio is operating on a single channel. In this scenario, a link is placed between two nodes if they are within their respective transmission ranges. This is the maximum achievable network connectivity since a single common channel is shared between all the nodes.
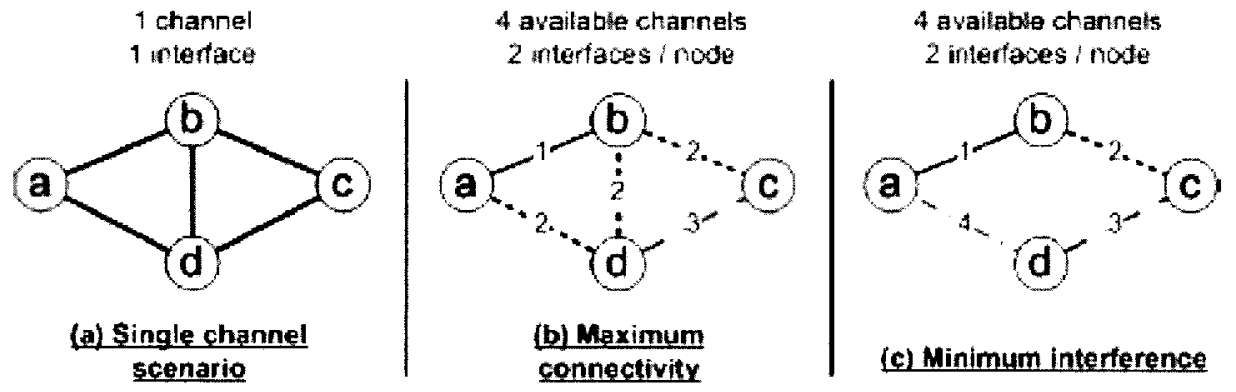

\section{Figure 3.3: Trade-off between connectivity and interference [19]}

\subsubsection{Maximum Connectivity in a Multi-radio Multi-channel Scenario}

Fig. $3.3 \mathrm{~b}$ shows the maximum network connectivity achieved by the assignment of different channels to the radios. Although, there is a direct communication link between every pair of neighbors, not all the links can be active simultaneously because of possible interference.

\subsubsection{Minimum Interference in a Multi-radio Multi-channel Scenario}

On the other hand, Fig. 3.3c shows how interference could be completely eliminated and all links can be simultaneously active. The compromise here is that there is no common channel and hence no link between neighbors $b$ and $d$.

The above example clearly illustrates that the goal of channel assignment is to achieve a balance between "minimizing interference and maximizing connectivity."

\subsection{Channel Assignment Problem}

In a typical WMN, the total number of radios is much higher than the number of available channels. Thus, many links between the mesh routers operate on the same set of channels and interference among transmissions on these channels decreases their utilization. Therefore, minimizing the effect of interference is required for the efficient reuse of the scarce radio spectrum. So, the key issue in a Multi-Radio Multi-Channel 
(MRMC) WMN architecture is the channel assignment problem, which involves assigning a channel to each radio of a MR in a way that minimizes interference on any given channel and guarantees connectivity between the mesh nodes [19].

\subsubsection{Objectives}

The CAA should satisfy the following two main goals:

1. Minimize co-channel interference between mesh routers

2. Ensure network connectivity

\subsubsection{Constraints}

Given the connectivity graph, the main challenge for CAA is to assign a channel to each radio in a way that minimizes interference between MRs and ensures connectivity between them. In order to achieve these goals, the CAA should satisfy the following requirements:

1. In order to communicate, a pair of nodes within transmission range of each other needs to have a common channel assigned to their end-point radios.

2. Links in direct interference range of each other should be assigned nonoverlapping channels.

3. The number of distinct channels that can be assigned to a mesh router is bounded by the number of radios it has.

4. The total number of non-overlapping channels is fixed.

5. Since the traffic in a WMN is directed to and from the gateway, the traffic flows aggregate at routers close to the gateway. Links that are expected to support heavy traffic should be given more bandwidth than others. In other words, these links should use a radio channel that is shared by fewer nodes. Therefore, priority in channel assignment should be given to links starting from the gateway based on the number of nodes that use a link to reach the gateway.

At first glance, the problem of assigning channels to links in a mesh network appears to be a graph-coloring problem. However, standard graph-coloring algorithms cannot satisfy all of its constraints, and it is NP-hard to find an optimal channel assignment to maximize the overall network throughput [18]. Also, the channel assignment problem for mesh networks is similar to the list coloring problem, which is NP-complete [28]. 


\section{CHAPTER 4}

\section{TOPOLOGY CONTROL AND CHANNEL ASSIGNMENT ALGORITHMS}

In this chapter, the graph theory representation of interference modeling in multiradio WMNs is discussed, and the network architecture of the proposed model is presented. The chapter presents the topology control and channel assignment algorithms for MRMC WMNs proposed in this thesis with details of their respective phases. The procedure of fault recovery with the proposed channel assignment algorithm is also presented in this chapter.

\subsection{Interference Modeling}

\subsubsection{Graph Theory Representation of a WMN}

At any point in time, the elements of a WMN can be represented as a graph [4]. A graph, $G$, is defined as a set of vertices $V$ and a set of edges $E$ and can be denoted as $G=$ $(V, E)$. The sets $V$ and $E$ have to be nonempty and finite. An edge is a link between two vertices, that joins the vertices $i$ and $j$, and is denoted by $(i, j)$. The vertices $i$ and $j$ are the end-vertices of this edge. If an edge exists between two vertices, then these two vertices are called adjacent or neighboring vertices of G. An example of such a graph is depicted in Figure 4.1.

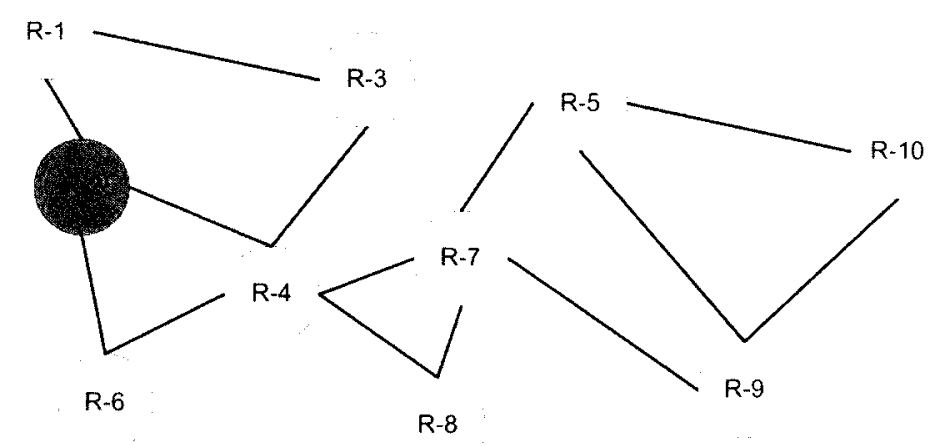

Figure 4.1: Graph representation of a WMN [4]

In a graphical representation of Multi-Radio-Wireless Mesh Network (MRWMN), each vertex in the graph represents a router in a MR-WMN, and each edge between two vertices represents a radio link between a pair of peer radio interfaces of two 
routers. A radio link is said to be operational between a pair of radio interfaces of two nodes if (a) these two radio interfaces operate on the same frequency channel and (b) the signals transmitted from one node can be received above a minimum required power threshold by the peer node, and vice versa. Two nodes are connected if there is a link between them.

\subsubsection{Connectivity Graph}

For modeling purposes, a WMN is generally considered in which the mesh routers are distributed on a plane, as shown in Figure 4.2. Each mesh router is equipped with one or multiple radios with omni-directional antennas. It is assumed that all radios are characterized by an identical transmission range $(\mathrm{R})$ and also by the same interference range ( $\left.\mathrm{R}^{\prime}\right)$.

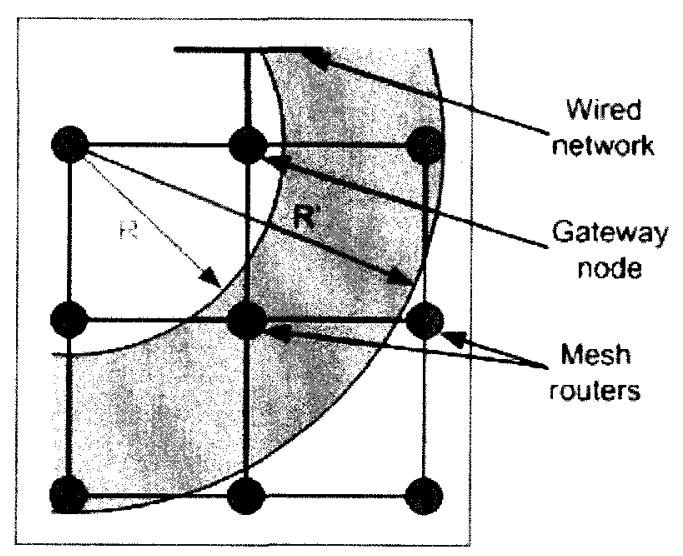

Figure 4.2: A connectivity graph [19]

\section{A. Transmission Range}

The Transmission Range (TR) is defined as the distance at which a neighbor can receive packet transmissions successfully. When a receiver is within the TR of two transmitters that are transmitting simultaneously, the packets are assumed to interfere with each other. This then leads to a collision at the receiver, and thus no packet is received successfully.

\section{B. Interference Range}

The Interference Range (IR) is defined as the distance at which packet transmission cannot be decoded successfully at the receiver. However, any new 
transmission from a router within IR from the receiver interferes with the packet reception. TR is smaller than IR ( $\left.<R^{\prime}\right)$ and IR is generally assumed to be twice the TR.

According to the above assumptions, connectivity between mesh routers can be modeled using an undirected graph referred to as a connectivity graph, G. As illustrated in Figure 4.2, two nodes in the connectivity graph are linked if they are located within TR of each other [19].

\subsection{Network Architecture}

In our proposed model, each mesh router is equipped with five radios which operate on IEEE 802.11a channels ( $5 \mathrm{GHz}$ band). One of these radios is used for control purpose while other radios are used for data traffic.

The control radios of all mesh nodes operate on the same non-overlapping IEEE 802.11a channel. Out of the 12 available non-overlapping 802.11a channels, channel 12 is used as the common control channel. Each mesh router is equipped with 4 data radios in order to utilize the remaining 11 non-overlapping channels available in IEEE 802.11a frequency band. Each MR communicates with its transmission range neighbors using these data radios for data transmission. So, each MR can have a maximum of 4 TR neighbors with whom it can communicate for data transmission which implies that the Maximum Node Degree (MND) per node is four.

The MND of 4 is selected in order to fully utilize the 11 available nonoverlapping 802.11a frequency channels. Results have shown that with 12 available nonoverlapping channels, the network throughput increases until a MND of four per node but saturates after that [18].

\subsection{Topology Control Algorithm}

The proposed Topology Control Algorithm (TCA) controls the network topology by selecting the nearest neighbors for each node in the network. The objective of the proposed TCA is to build a connectivity graph with a small node degree to mitigate the co-channel interference and enhance spatial channel reuse as well as preserve network connectivity with the use of minimal power, as less transmit power translates to less interference. 


\subsubsection{Gateway Advertisement Process}

Initially, the gateway broadcasts a "Hello" message, using its control radio on the control channel, announcing itself as the gateway. Each mesh node that receives this Hello message over its control radio broadcasts it again and in this way, this Hello message is flooded throughout the mesh network. The Hello message contains a hopcount field that is incremented at each hop during its broadcast. So, a mesh node may receive multiple copies of the Hello message over its control radio. However, distance of a mesh node from the gateway is the shortest path length (shortest hop count) of the Hello message received by a mesh node through its control radio over different paths. In this way, each mesh node knows the next hop to reach the gateway using its control radio.

\subsubsection{Assumptions}

The proposed TCA assumes the following:

- Each node knows its location.

- Each node uses an omni-directional antenna for both transmission and reception.

- Each node is able to adjust its own transmission power.

- The maximum transmission power is the same for all nodes and hence, the maximum TR for any pair of nodes to communicate directly is also the same.

Note that all nodes start with the maximum transmission power, and that the initial topology graph created, when every node transmits with full power, is strongly connected.

\subsubsection{Phases of TCA}

The proposed TCA consists of the following five phases:

\section{A. Exchange of Information Between Nodes}

In the first exchange, each node broadcasts a HELLO message at maximum transmission power containing its node ID and the node position.

\section{B. Building the Maximum Power Neighbor Table (MPNT)}

From the information in the received HELLO messages, each node arranges its neighboring nodes in the ascending order of their distance. The result is the Maximum Power Neighbor Table (MPNT). Then, each node sends its MPNT along with its position and node ID to the gateway node using its control radio over the control channel. 


\section{Building the Direct Neighbor Table (DNT)}

For each node in the network, the gateway builds a Direct Neighbor Table (DNT). Based on information in the MPNT of node $v$ and the MPNTs of its neighbors, if

(a) node $w$ is in the MPNT of node $v$, and

(b) node $w$ is closer to any other node $y$ in the MPNT of node $w$ than to node $v$, then gateway eliminates node $w$ from the MPNT of node $v$.

If, after removing nodes from the MPNT of node $v$, the remaining number of nodes in the MPNT of node $v$ is less than " $x$," then the gateway selects " $x$ " nearest nodes as neighbors of node $v$ which results in the DNT. However, if after removing nodes from the MPNT of node $v$, the remaining number of nodes is greater than or equal to " $x$," then the result is the DNT.

This algorithm is called Select $x$ for less than $x$ TCA where $x$ is a positive integer. The Select $x$ for less than $x$ TCA ensures that each node has at least $x$ neighbors, as shown in Figure 4.3.

\section{Converting into Bi-directional Links}

For each node in the network, the gateway converts the uni-directional links in the DNT of a node into bi-directional links. For each uni-directional link, this is done by adding a reverse link in the DNT of the neighboring node. This converts the DNT into Bi-directional DNT. This results in the Final Neighbor Table (FNT).

\section{E. Calculating the Minimum Power Required}

For each node in the network, the gateway calculates the minimum power, $P_{\min }$, required to reach each of the nodes in the FNT of a node, using the appropriate propagation model formulas.

\subsubsection{Propagation Models}

The free space model is used for short distances and the two ray ground reflection model is used for longer distances, depending on the value of the Euclidean distance in relation to the cross-over distance. The cross-over distance is calculated by [29]

$$
\text { Cross_over_dist }=\frac{4 \pi h t h r}{\lambda},
$$

where $h_{t}$ and $h_{r}$ are the antenna heights of the transmitter and receiver, respectively. If the distance between two nodes is less than the cross over distance, i.e. $d(u, v)<$ 
Cross_over_dist, Free Space propagation model is used, whereas if $d(u, v)>$ Cross_over_dist, Two-ray propagation model is used. The minimum power for the freespace propagation model is calculated by [29]

$$
P_{\min }=\frac{R x T h r e s h(4 \pi d)^{2}}{G_{t} G_{r} \lambda^{2}} .
$$

The minimum power for the two-ray propagation model is calculated by [29]

$$
P_{\min }=\frac{R x \operatorname{Thresh}(d)^{4}}{G_{t} G_{r} h_{t}{ }^{2} h_{r}{ }^{2}},
$$

where $G_{t}$ and $G_{r}$ are transmitter and receiver antenna gain respectively, and RxThresh is power threshold required by radio interface of receiving node to correctly understand the message.

\subsection{Channel Assignment Algorithm}

\subsubsection{Interference-range Edge Coloring}

If $K$ be the number of available colors (channels), then for $K \geq 4$, the distance-2 edge coloring problem, also known as strong edge coloring problem, is NP-complete [30]. A distance-2 edge coloring of a graph $G$ is an assignment of colors to edges so that any two edges within distance 2 of each other have distinct colors. Two edges of $G$ are within distance 2 of each other if either they are adjacent, as shown in Figure 4.4a or there is some other edge that is adjacent to both of them, as shown in Figure 4.4b. The distance-2 edge coloring has been used in [31] for channel assignment, where the authors have described the interference model as two-hop interference model. In this model, two edges interfere with each other if they are within two-hop distance. In other words, two edges $e_{1}$ and $e_{2}$ cannot transmit simultaneously on the same channel if they are sharing a node or adjacent to a common edge.

To minimize co-channel interference in a wireless mesh network, it is necessary to assign channels to links such that links within interference range of each other are assigned different channels (colors). This problem can be termed as interference-range edge coloring, and the corresponding interference model can be called interference-range interference model. In a grid topology where links are of equal length, the interferencerange edge coloring is similar to distance- 2 edge coloring, as shown in Figure 4.5a. The 
channel assigned to link $l_{1}$ cannot be assigned to links $l_{2}$ and $l_{3}$ as they are within the interference range of link $l_{1}$. Note that $l_{2}$ and $l_{3}$ are also within two-hop distance of $l_{1}$.

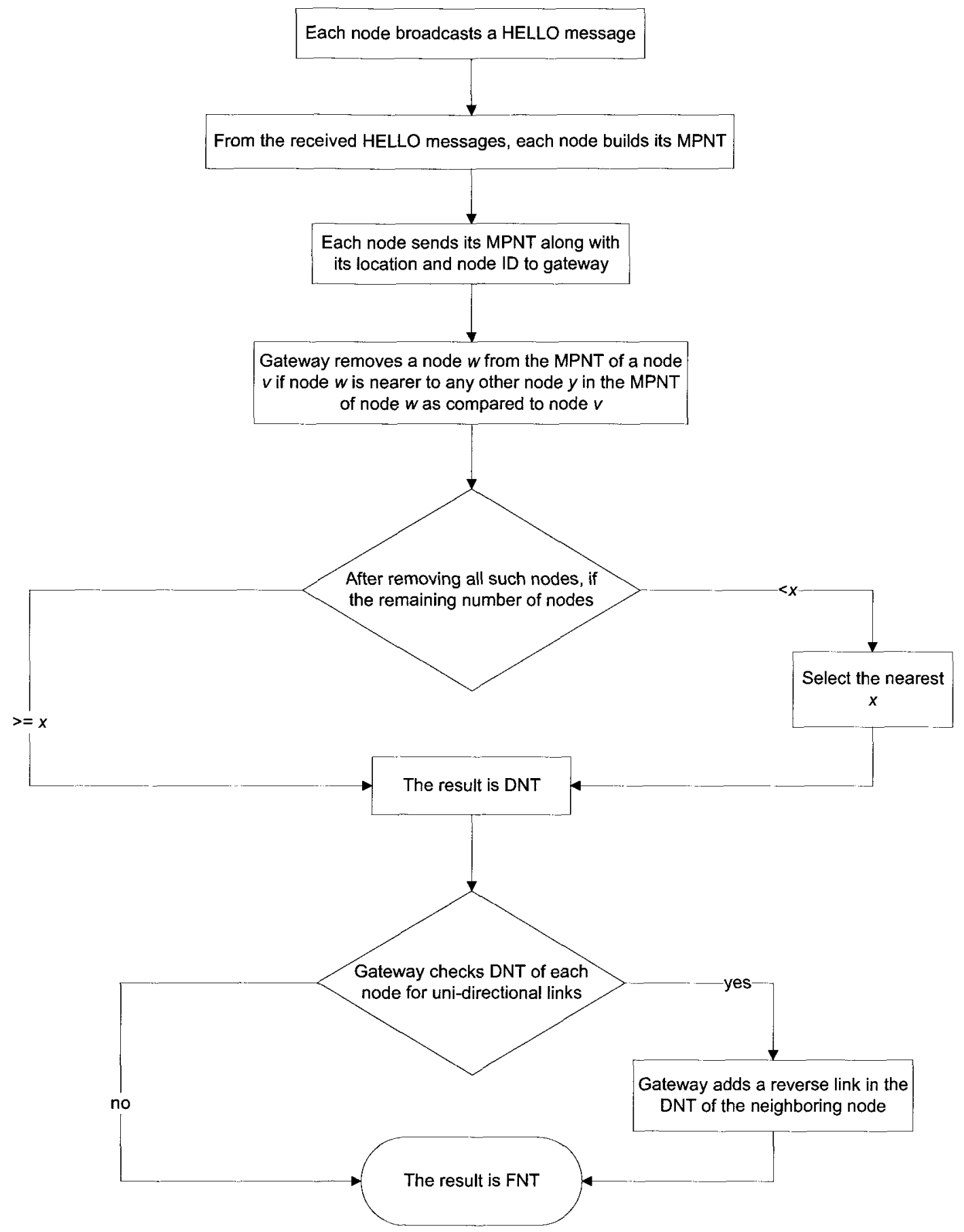

Figure 4.3: Select $x$ for less than $x$ TCA

However, in a random topology where links are of different lengths due to the random nature of the topology, the interference-range edge coloring can be harder than distance- 2 edge coloring as shown in Figure $4.5 \mathrm{~b}$. In this case, the channel assigned to 
link $l_{1}$ cannot be assigned to links $l_{2}, l_{3}$ and $l_{4}$ as they are within the interference range of link $l_{1}$. Note that $l_{2}, l_{3}$ and $l_{4}$ are within three-hop distance of $l_{1}$.

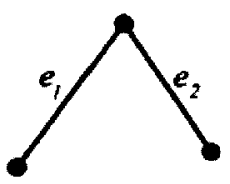

(a)

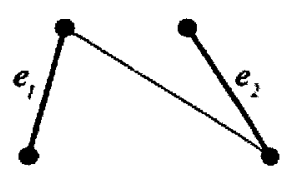

(b)

Figure 4.4: Two edges at distance-2 of each other [30]

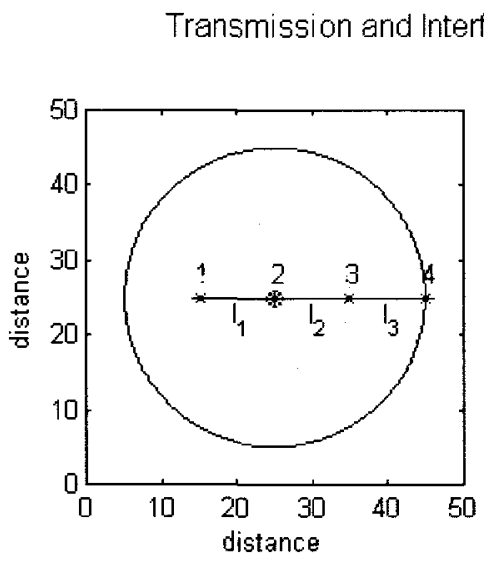

(a) Grid Topology

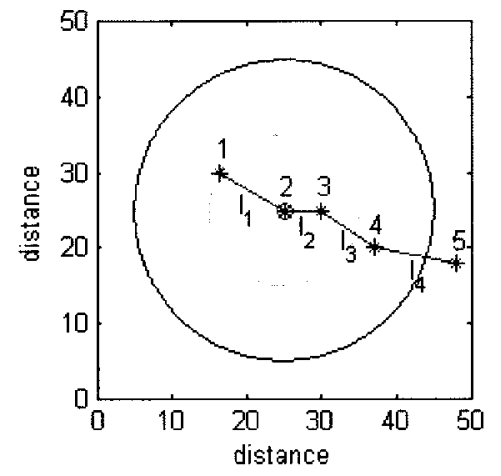

(b) Random Topology

Figure 4.5: Interference-range edge coloring

In the network model proposed in this thesis, the number of available channels (colors) is 11 which means that $K=11$. Based on its similarity to distance-2 edge coloring problem which is NP-complete for $K \geq 4$, the interference-range edge coloring problem is, therefore, also NP-complete.

Therefore, I propose an approximate algorithm for channel assignment. The proposed channel assignment algorithm, TICA, is shown in Figure 4.6 and has the following phases.

\subsubsection{Phases of TICA}

\section{A. Topology Control}

In order to create the network connectivity graph with the aim of reducing the interference between MRs, network topology is controlled using the topology control algorithm. 
All nodes send their MPNTs to the gateway using their control radio. Note that in order to send its MPNT to the gateway, each mesh node knows the next hop to reach the gateway using its control radio via the "gateway advertisement process." The gateway starts with the Select 1 for less than 1 TCA and builds the FNTs of all nodes.

\section{B. Connectivity Graph}

Based on the FNTs of all nodes, the gateway builds the connectivity graph and checks the resulting network for connectivity. A topology is said to be connected if the gateway can reach any node in the connectivity graph directly or through intermediate hops.

If the resulting network is not connected, the gateway moves to the next higher TCA by incrementing $x$ in the Select $x$ for less than $x$ TCA, builds the connectivity graph and checks the resulting network for connectivity. The gateway keeps on moving to a higher TCA until its finds that the network resulting from the connectivity graph is connected.

\section{Minimum Power-based Shortest Path Tree with a MND of Four}

After ensuring that the connectivity graph is connected, the gateway builds the Shortest Path Tree (SPT), using Dijkstra's algorithm [32], based on the connectivity graph. The metric for path selection is minimum power.

The node degree is defined as the number of TR neighbors of a node. The number of TR neighbors of a mesh router is bounded by the number of its radios and each node has four data radios. So, if any node in the shortest path tree has more than four links, the gateway selects those four links for that node which have the minimum weight and sets the weight of all other links to infinity. In other words, the gateway ensures that each node can have a maximum of four TR neighbors and builds a Minimum Power-based SPT (MPSPT) with a MND of 4 per node. The gateway checks the resulting MPSPT graph for connectivity. If the resulting MPSPT graph is not connected, the gateway moves to a higher TCA.

Once the MPSPT graph is determined, the gateway has to assign channels to links of the MPSPT. Now, the objective is to assign channels to the links of the MPSPT such that the interference between simultaneous transmissions on links operating on the same channel is minimized and the overall network throughput is maximized. 


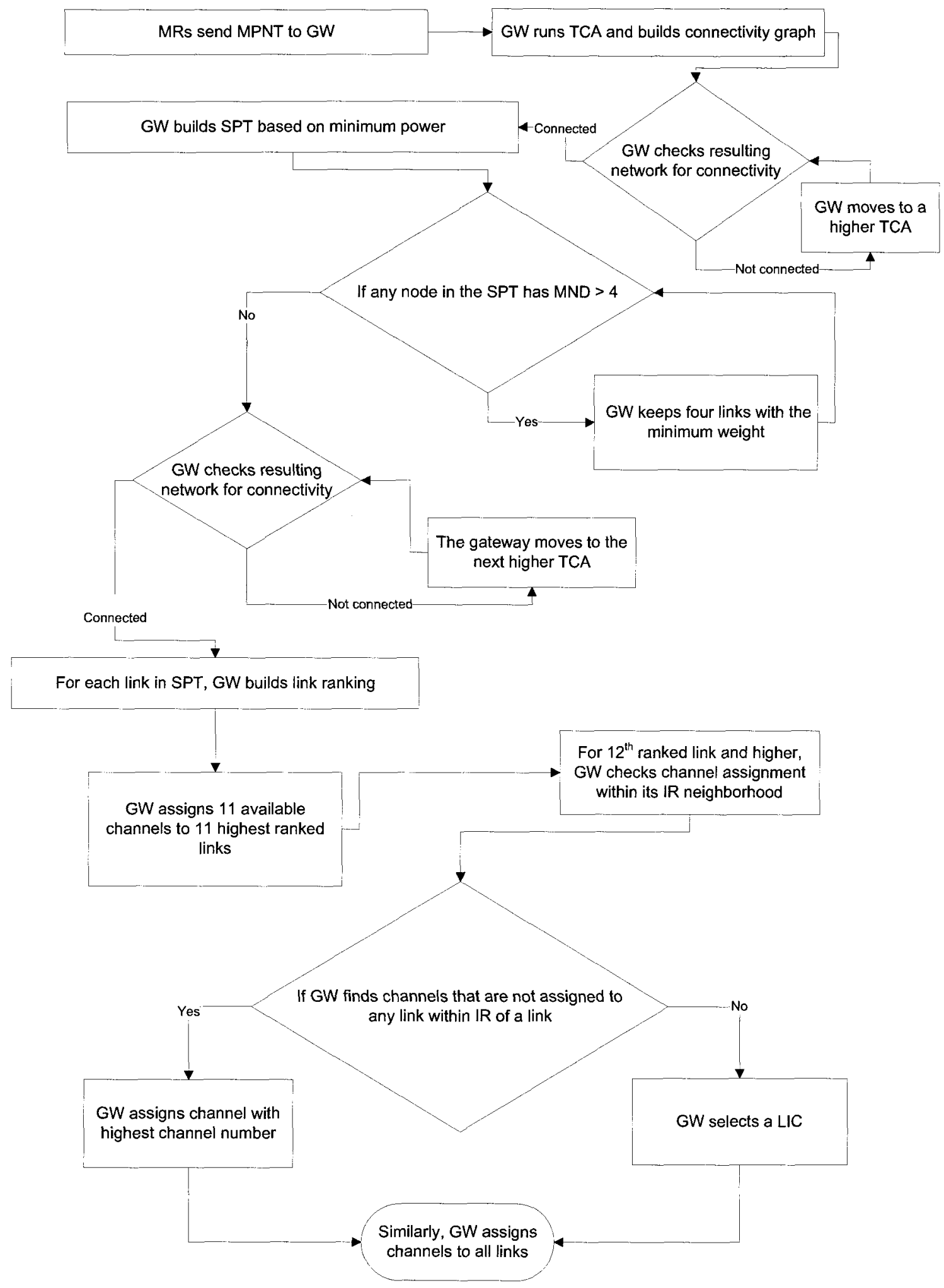

Figure 4.6: Topology-controlled Interference-aware Channel-assignment Algorithm (TICA) 


\section{Link Ranking}

In order to assign channels to the links of the MPSPT graph, each link is assigned a ranking by the gateway. The ranking associated with each link is derived from the number of nodes that use a link to reach the gateway node. If $l$ is link and $n$ is node using link $l$ to reach the gateway, then rank of link $l$, i.e. $r_{l}$, is given by

$$
r_{l}=\sum_{n=1}^{N} I_{n, l},
$$

where $N$ is the total number of nodes in the network. $I_{n, l}$ is 1 if node $n$ is using link $l$ and 0 otherwise.

In the case of two or more links that have the same rank, the link whose power of the farthest node to the gateway is smaller is given priority in channel assignment. If there are some links that still have the same rank, the link with smaller node IDs is given priority in channel assignment.

\section{E. Channel Assignment}

The gateway assigns a channel to each link in the order of its rank, and it begins with assigning the 11 available non-overlapping channels to the 11 highest-ranked links such that Channel 1 is assigned to the highest-ranked link. For the $12^{\text {th }}$-ranked link and onwards, the gateway checks the channel assignment of all links within the interference range of both nodes that constitute that link.

\section{i) Non-conflicting Channel}

Out of the 11 available channels, channels which are not assigned to any link within the interference range of both nodes that constitute the $12^{\text {th }}$-ranked link are termed as non-conflicting channels. If the gateway finds one or more non-conflicting channels, it assigns that channel from the unassigned non-conflicting channels to the $12^{\text {th }}$-ranked link which has the highest channel number.

\section{ii) Least Interfering Channel}

If the gateway cannot find any channel among the 11 available channels that is not assigned to any link within the interference range of both nodes that constitute the $12^{\text {th }}$-ranked link, it selects the least interfering channel and assigns it to that link. A Least 
Interfering Channel (LIC) is a channel which causes minimum interference within the interference range of both nodes that constitute the $12^{\text {th }}$-ranked link.

\section{iii) Interference Level}

In order to find out the LIC, the gateway builds the interference level for all the 11 channels. The LIC is the channel with the minimum interference level, which means that assigning this channel to the $12^{\text {th }}$-ranked link would result in minimum interference in the network.

In order to build the IL for Channel One, the gateway finds all links within the interference range of each of the two nodes that constitute the $12^{\text {th }}$-ranked link that use Channel One, and calculates IL of each link based on its rank and distance from a node of the $12^{\text {th }}$-ranked link. It sums up individual ILs of all links that use Channel One within the interference range of each of the two nodes that constitute the $12^{\text {th }}$-ranked link, to find out total IL for Channel One. This is done by

$$
(I L)_{i}=\sum_{m}\left(\frac{r_{m}}{R}\right)\left(\frac{1}{d_{m}^{\alpha}}\right),
$$

where $i$ is the channel that has value between 1 and 11 , $(I L)_{i}$ is interference level of channel $i$,

$m$ is a link using channel $i$ that is within the interference range of a node of the $12^{\text {th }}$ ranked link,

$r$ is the rank of link $m$,

$R$ is the maximum rank assigned to a link, $d$ is distance from a node of link $m$ to a node of the $12^{\text {th }}$-ranked link, and $\alpha$ is the path loss exponent and is 2 or 4 , depending on cross over distance.

If a link is emanating from either of the two nodes that constitute the $12^{\text {th }}$-ranked link and a channel has been assigned to that link, then the IL for this channel is set to infinity.

The LIC is the channel with the minimum interference level and is selected by

$$
(I L)_{L I C}=\min \left[(I L)_{1},(I L)_{2}, \ldots \ldots . .,(I L)_{11}\right] \text {. }
$$

Similarly, the gateway assigns channels to all the links of the MPSPT. 


\section{iv) Channel Assignment and Routing Message}

Using its control radio, the gateway then sends each mesh node the Channel Assignment and Routing Message (CARM). For each channel assigned to a mesh router, CARM contains the channel number and the neighbor node to communicate with, using this channel. The CARM also contains the next hop to reach the gateway for data traffic. Based on the channel assigned to a mesh router to communicate with a neighbor and its distance to that neighbor, the mesh router applies power control and adjusts its transmission power accordingly by using either the free space propagation model given by (2) or the two-ray propagation model given by (3), depending on the cross-over distance which is calculated using (1).

\subsection{Failure Recovery Mechanism of TICA}

When a node fails, nodes in its sub-tree lose their connectivity to the gateway and hence the Internet through the wired world. TICA supports automatic and fast failure recovery and reorganizes the network to bypass the failed node and to restore the connectivity.

In case of node failure, the FRM of TICA which is initiated by the gateway has following phases, as shown in Figure 4.7.

\section{A. Periodic Keep-Alive Messages}

All nodes send periodic "keep-alive" messages to the gateway on the control channel using their control radios. The keep-alive message from a node tells the gateway that the node is active.

\section{B. Topology Control}

If a gateway does not receive three consecutive keep-alives from a node $z$, then it concludes that node $\mathrm{z}$ has failed and is no longer active. The gateway then deletes the MPNT for this node and deletes node $\mathrm{z}$ from the MPNT of all its neighboring nodes. Note that the gateway has MPNTs of all nodes, as all nodes sent their MPNTs to the gateway during the setup phase. The gateway builds the FNTs for all nodes using the Select $x$ for less than $x$ TCA. 


\section{Connectivity Graph}

Based on the FNTs of all nodes, the gateway builds the connectivity graph and checks the resulting network for connectivity. If the resulting network is not connected, the gateway moves to a higher TCA.

\section{Minimum Power-based SPT with a MND of Four}

After ensuring that the connectivity graph is connected, the gateway builds the MPSPT with a MND of four. Again, the gateway checks the resulting MPSPT graph for connectivity. If the resulting MPSPT is not connected, the gateway moves to a higher TCA.

\section{E. Link Ranking}

After ensuring that the MPSPT is connected, the gateway builds the link ranking for all links of the MPSPT.

\section{F. Channel Assignment}

Based on the link ranking, the gateway assigns the channels to all links of the MPSPT.

The gateway then sends the new CARM to all nodes in the network on the control channel. Each MR receives the CARM on the control channel over its control radio and compares the existing CA with the updated CA. It switches its radios to new channels in case the new CA is different from the old one. Based on the channel assigned to a MR to communicate with a neighbor and its distance to that neighbor, the MR applies power control and adjusts its transmission power accordingly. This is done by calculating the required controlled transmission power by using either the free space propagation model using (2) or the two-ray propagation model using (3), depending on the cross-over distance, which is calculated using (1). Each MR also updates its next hop required to reach the gateway for data traffic. 


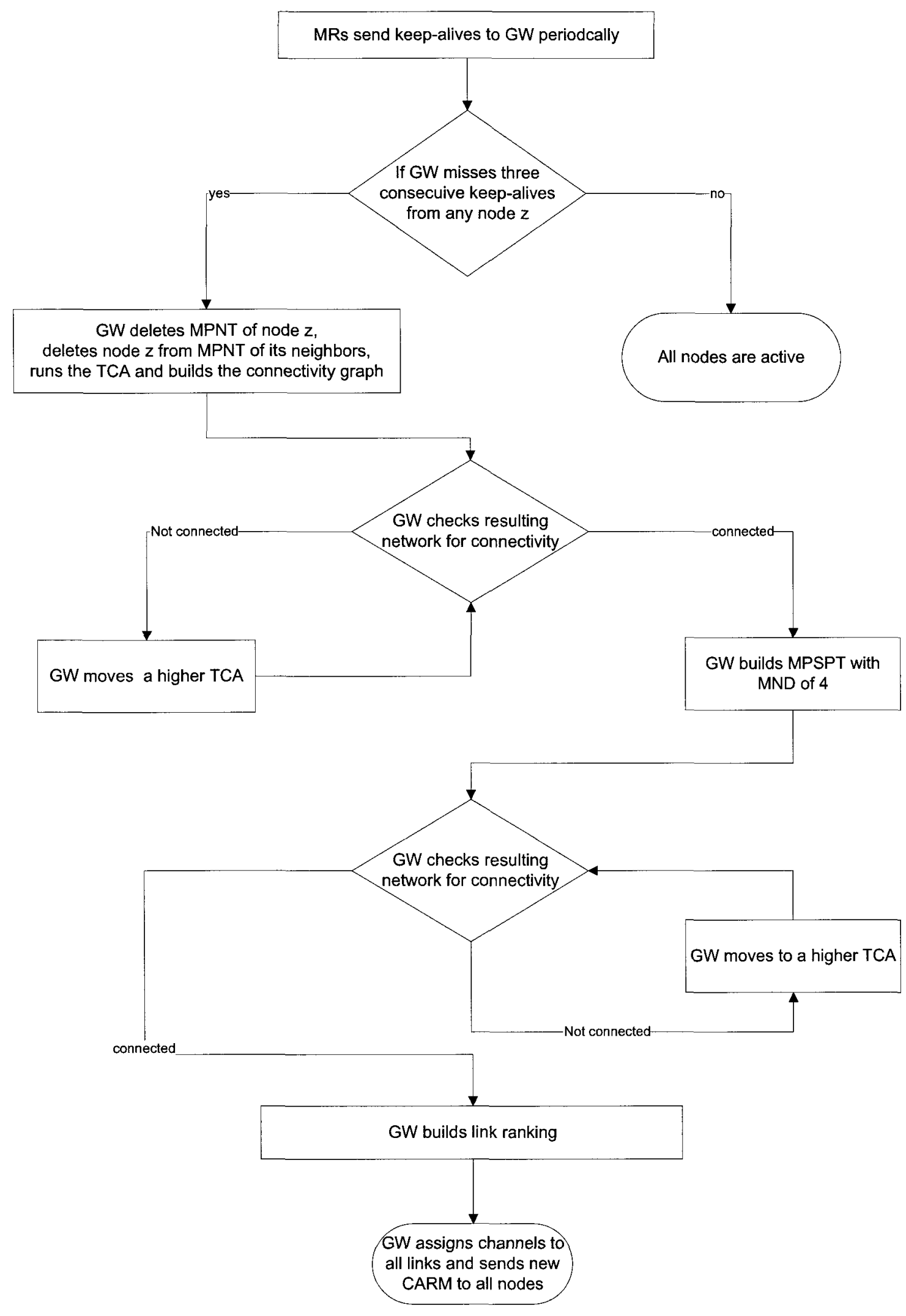

Figure 4.7: Failure Recovery Mechanism of TICA 


\section{CHAPTER 5}

\section{PERFORMANCE EVALUATION}

In this chapter, the performance evaluation of the proposed channel assignment algorithm is provided. Different topologies used for performance evaluation are presented, and details on the working of different phases of TICA and FRM of TICA are discussed, with examples. The objective of the simulation-based evaluation is to understand the behavior of TICA in small-scale and large-scale networks.

The performance of the proposed algorithm for MRMC WMNs, TICA, is compared against a "Single-Radio Single-Channel" (SRSC) scheme and a "Common Channel Assignment" (CCA) scheme for multi-radio mesh nodes. In the CCA scheme [20], all mesh routers have four radio interfaces. The first radio on all nodes is tuned to the first non-overlapping channel; the second radio is tuned to the second nonoverlapping channel, and so on.

\subsection{Simulation Environment}

For the performance evaluation via throughput analysis, NS2 (version 2.30) [33] simulation tool is used. However, MATLAB [34] is used to generate the power controlled topology, the MPSPT graph, the link ranking of the MPSPT and the channel assignment for the links of the MPSPT.

Multi-interface wireless mesh nodes are created in NS2 by modifying the built-in IEEE 802.11 node model in NS2, using the procedure given in [35]. Based on the channel assignment by the gateway, the radio interfaces are configured for each node and the transmission power for each radio of each mesh node is set accordingly. All the mesh nodes at the periphery of the network send traffic to the gateway. Each of these nodes generates an $8 \mathrm{Mbps}$ Constant Bit Rate (CBR) traffic stream consisting of 1024 byte packets, and sends data to the gateway node at the same time. They do not stop transmitting until the end of the simulation. So, this is a scenario in which multiple flows within the mesh network interfere with each other.

All radios are IEEE 802.11a radios and support 12 channels. The first 11 nonoverlapping channels are used by the data radios, whereas the $12^{\text {th }}$ channel is used by the 
control radio on each node. If the distance between the nodes is less than the cross-over distance, free space propagation model is used; if the distance between the nodes is greater than the cross-over distance, two-ray propagation model is used. As shown in Tables 5.1 and 5.2 [5], the minimum receiver sensitivity (RxThresh) is set to $-65 \mathrm{dBm}$ $\left(3.16227 \times 10^{-10}\right.$ Watts) in order to achieve a maximum data rate of $54 \mathrm{Mbps}$ supported by IEEE 802.11a.

Table 5.1: IEEE 802.11a Receiver Requirements [5]

\begin{tabular}{|c|c|c|c|c|c|c|}
\hline Modulation & $\begin{array}{l}\text { Codiug } \\
\text { late } \\
\text { (R) }\end{array}$ & $\begin{array}{l}\text { Adjacent } \\
\text { channel } \\
\text { rejection } \\
\text { (dB) }\end{array}$ & $\begin{array}{l}\text { Alteruate } \\
\text { adjacent } \\
\text { channel } \\
\text { rejection } \\
\text { (dB) }\end{array}$ & $\begin{array}{c}\text { Minimum } \\
\text { sensitivity } \\
\text { (dBm) } \\
(20 \mathrm{MHz} \\
\text { channel } \\
\text { spacing) }\end{array}$ & $\begin{array}{c}\text { Minimum } \\
\text { sensitivity } \\
\text { (dBm) } \\
\text { (10 MHz } \\
\text { clannel } \\
\text { spacing) }\end{array}$ & $\begin{array}{l}\text { Minimum } \\
\text { sensitivity } \\
\text { (dBm) } \\
\text { (5 } \mathrm{MIHz} \\
\text { channel } \\
\text { spacing) }\end{array}$ \\
\hline BPSK & 12 & 16 & 32 & -82 & -85 & $-\$ 8$ \\
\hline BPSK & 34 & 15 & 31 & -81 & -84 & -8 \\
\hline QPSK & 12 & 13 & 29 & -9 & -82 & -85 \\
\hline QPSK & 34 & 11 & 27 & -- & -80 & -83 \\
\hline 16-QAM & 12 & 8 & 24 & -4 & -7 & -80 \\
\hline 16-QAM & 34 & 4 & 20 & -70 & --3 & --6 \\
\hline 64-QAM & 23 & 0 & 16 & -66 & -69 & -72 \\
\hline 64-QAM & 34 & -1 & 15 & -65 & -68 & -1 \\
\hline
\end{tabular}

Table 5.2: IEEE 802.11a Modulation Parameters [5]

\begin{tabular}{|c|c|c|c|c|c|c|c|}
\hline Modulation & $\begin{array}{l}\text { Coding } \\
\text { late } \\
(R)\end{array}$ & $\begin{array}{c}\text { Coded bits } \\
\text { per } \\
\text { subcarrier } \\
\left(N_{B P S C}\right)\end{array}$ & $\begin{array}{c}\text { Coded } \\
\text { bits per } \\
\text { OFDMI } \\
\text { symbol } \\
\left(N_{C B P S}\right)\end{array}$ & $\begin{array}{c}\text { Data bits } \\
\text { per } \\
\text { OFDM } \\
\text { symbol } \\
\left(N_{D B P S}\right)\end{array}$ & $\begin{array}{c}\text { Data l'ate } \\
\text { (Mb/s) } \\
\text { (20 MHz } \\
\text { channel } \\
\text { spacing) }\end{array}$ & $\begin{array}{c}\text { Data mate } \\
\text { (Mb/s) } \\
\text { (10 MHz } \\
\text { channel } \\
\text { spacing) }\end{array}$ & $\begin{array}{c}\text { Data rate } \\
\text { (IIbs) } \\
\text { (5 MHz } \\
\text { channel } \\
\text { spacing) }\end{array}$ \\
\hline BPSK & 12 & 1 & 48 & 24 & 6 & 3 & 1.5 \\
\hline BPSK & 34 & 1 & 48 & 36 & 9 & 4.5 & 2.25 \\
\hline QPSK & 12 & 2 & 96 & 48 & 12 & 6 & 3 \\
\hline QPSK & 34 & 2 & 96 & -2 & 18 & 9 & 4.5 \\
\hline 16-QAM & 12 & 4 & 192 & 96 & 24 & 12 & 6 \\
\hline 16-QAM & 34 & 4 & 192 & $1+4$ & 36 & 18 & 9 \\
\hline 64-QAM & 23 & 6 & 288 & 192 & 48 & 24 & 12 \\
\hline 64-QAM & 34 & 6 & 288 & 216 & 54 & $2-$ & 13.5 \\
\hline
\end{tabular}

In order to achieve a strongly connected topology, the maximum transmission power for all the radios is set to $27 \mathrm{dBm}$. The maximum power transmission range is 164 meters and the maximum power interference range is 328 meters. RTS/CTS is disabled. 
Note that in the CCA and SRSC schemes, the mesh nodes do not control their power, transmit with the same maximum power $(27 \mathrm{dBm})$ and use AODV (Ad hoc On-Demand Distance Vector) [36] routing protocol as their routing agent.

\subsection{Network Topologies}

Three topologies are used in the evaluation. Each topology consists of 36 mesh nodes distributed in an area of $500 \times 500$ meters.

Topology 1 is a grid topology; Topology 2 is a randomly generated topology while in Topology 3, called controlled random, the physical terrain is divided into a number of cells and a mesh node is placed randomly within each cell.

Grid Topology (GT), as shown in Figure 5.1, is used to evaluate TICA in a densely populated topology.

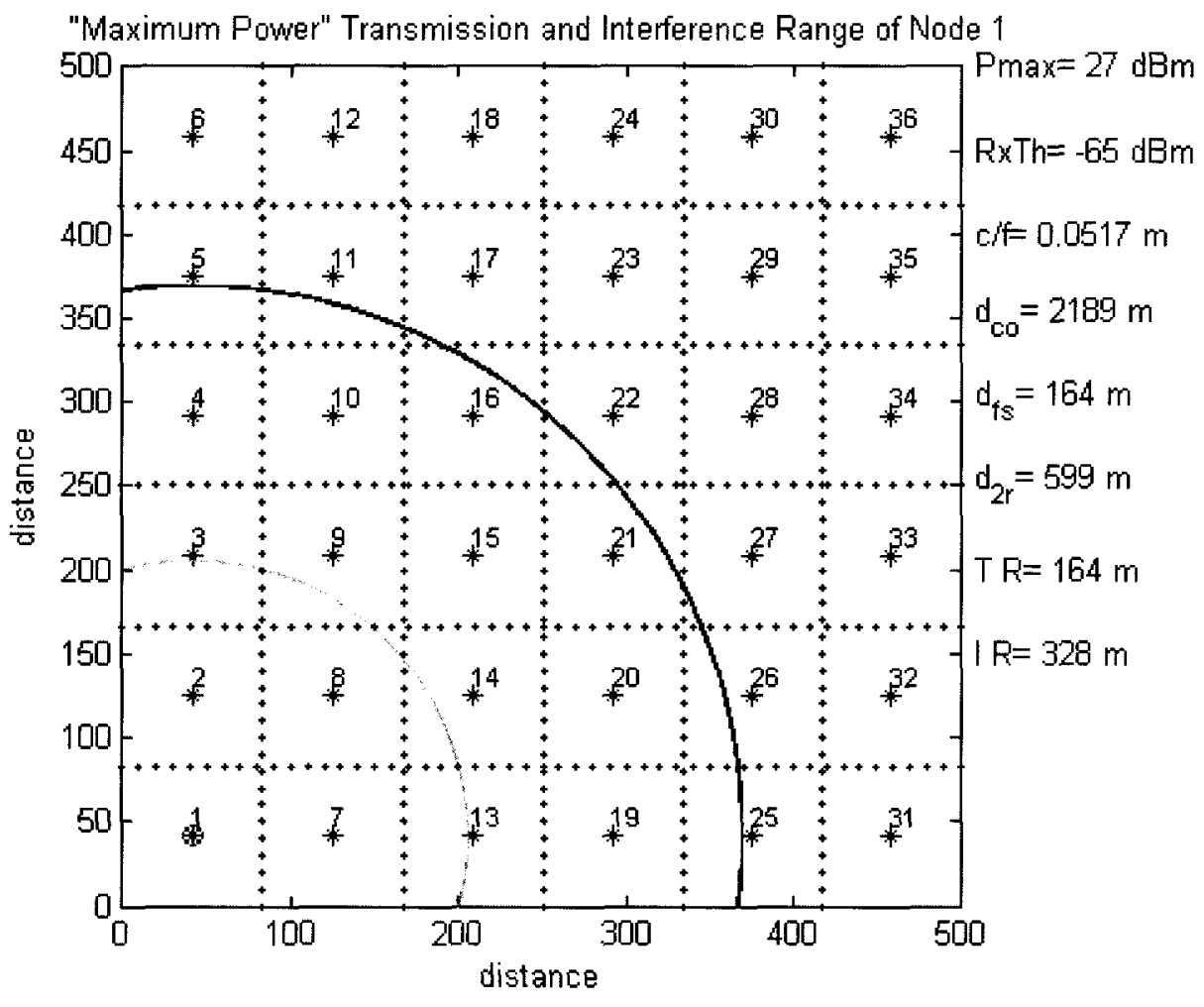

Figure 5.1: Grid Topology

Random Topology (RT), as shown in Figure 5.2, is used to evaluate TICA in an unplanned deployment of randomly and uniformly distributed mesh nodes.

Controlled Random Topology (CRT), as shown in Figure 5.3, is used to reflect real-world deployments where mesh routers are uniformly distributed for maximum coverage. 


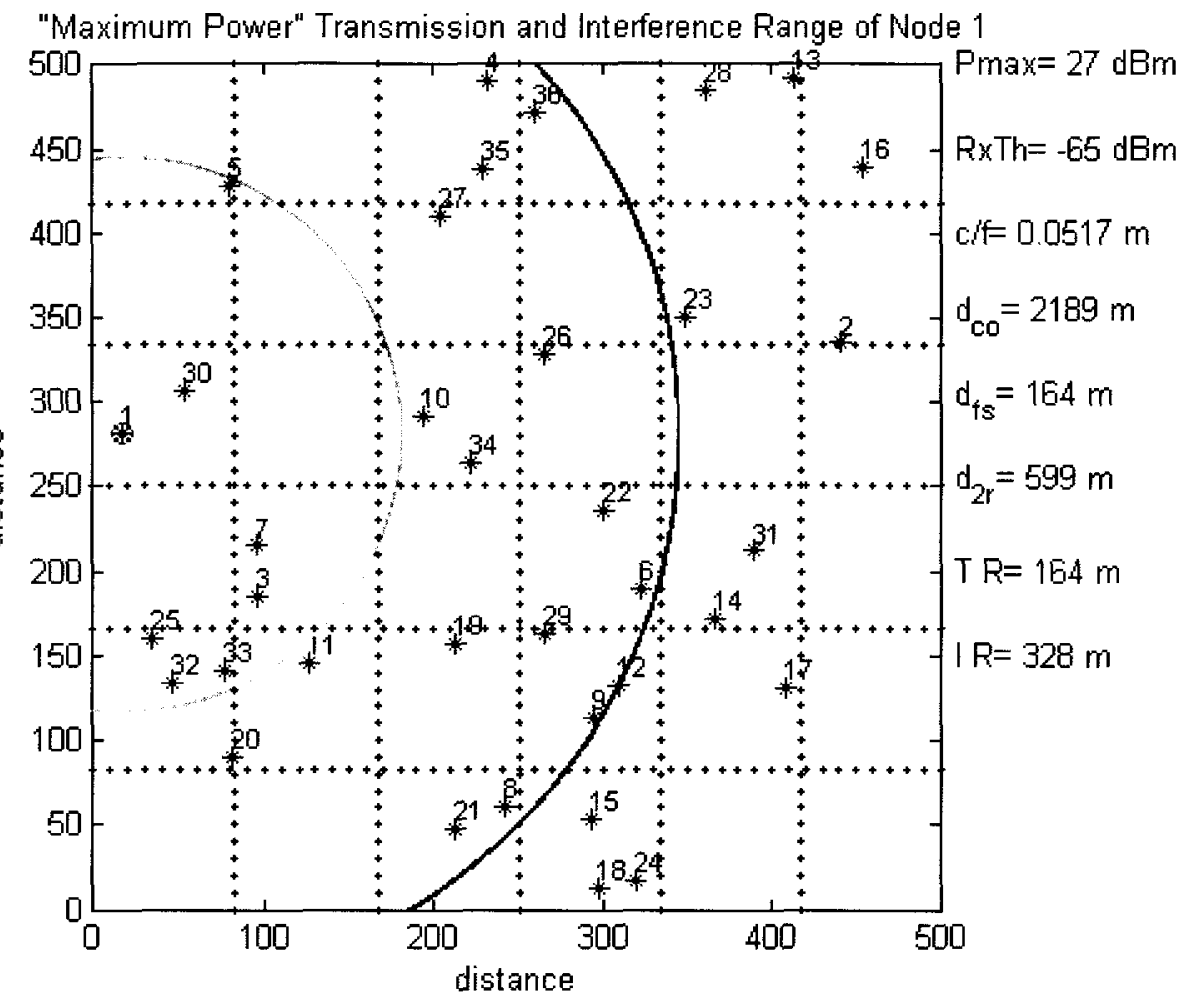

Figure 5.2: Random Topology

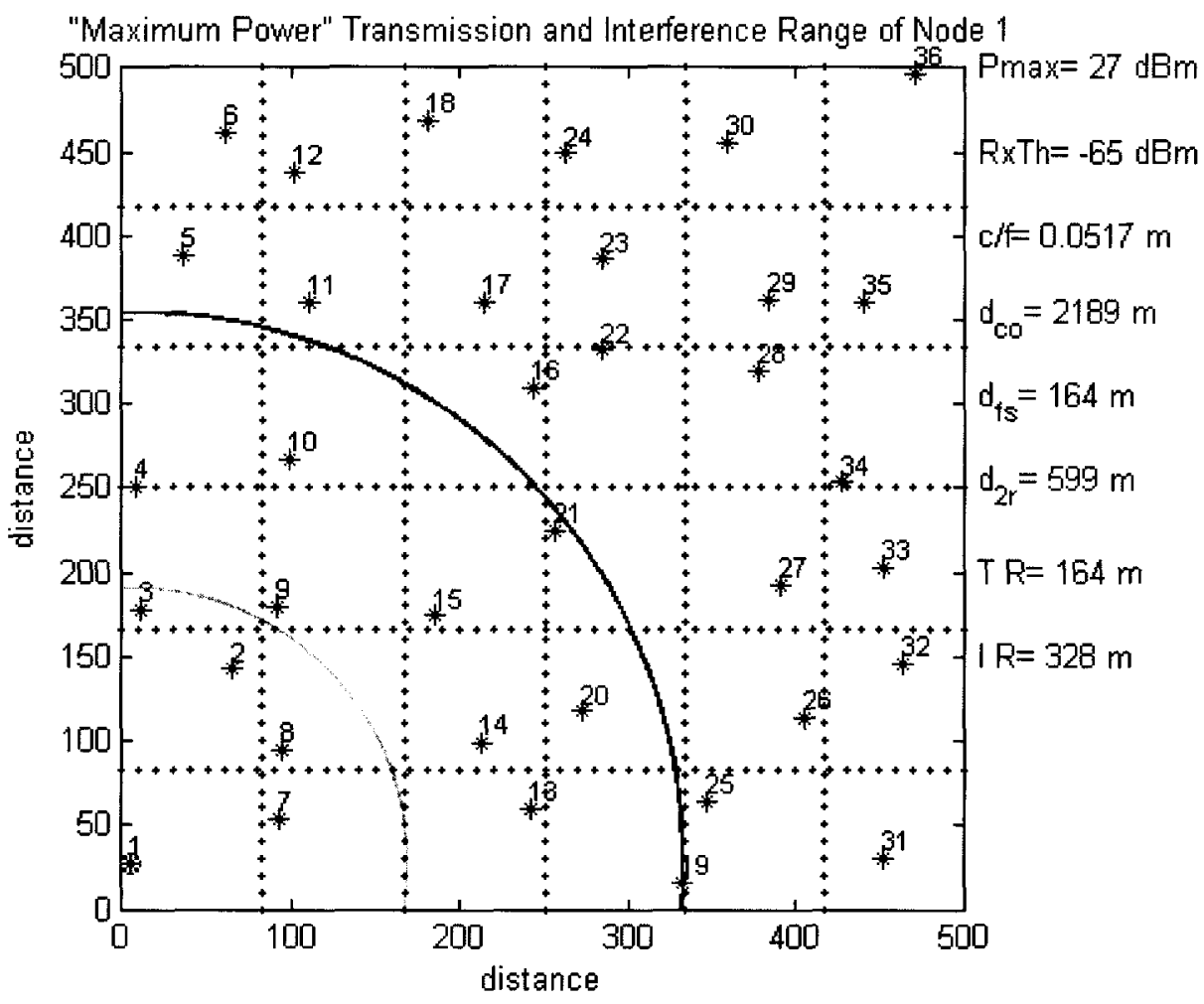

Figure 5.3: Controlled Random Topology 


\subsection{Working of TICA}

The working of different phases of TICA is explained below, with examples. The random topology shown in Figure 5.2 is considered for this purpose.

\subsubsection{Topology Control Phase}

\section{A. Exchanging Hello Messages}

Each node broadcasts a HELLO message at maximum transmission power containing its node ID and node position. As shown in Figure 5.4, Node 1 receives Hello messages from all nodes in its maximum power transmission range.

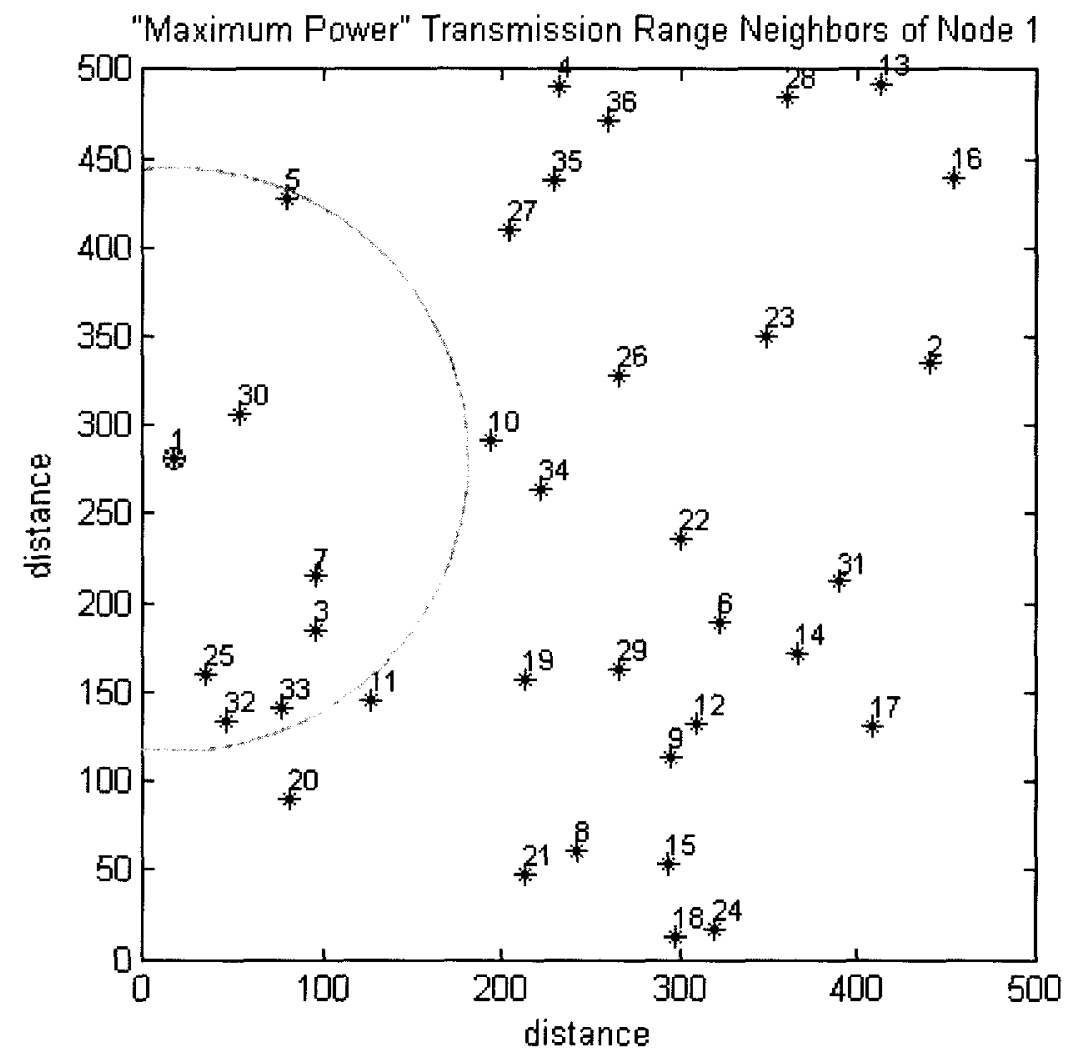

Figure 5.4: Maximum Power Transmission Range neighbors of Node 1

Table 5.3 shows the node ID and node location of the maximum power transmission range neighbors obtained, from the received Hello messages, by Node 1.

Similarly, from the received hello messages, each node in the network obtains the node ID and node location of its maximum power transmission range neighbors. 
Table 5.3: Neighbor information received by Node 1

\begin{tabular}{|c|c|c|}
\hline Neighbor Node ID & X coordinate & Y coordinate \\
\hline 3 & 96 & 185 \\
\hline 5 & 79 & 428 \\
\hline 7 & 96 & 215 \\
\hline 25 & 35 & 160 \\
\hline 30 & 53 & 306 \\
\hline 32 & 46 & 134 \\
\hline 33 & 77 & 141 \\
\hline
\end{tabular}

\section{B. Building the MPNT}

In order to build its MPNT, Node 1 calculates its distance to each of its maximum power transmission range neighbors. For example, Node 1 calculates its distance to Node 3 using the distance formula as follows:

$$
\text { distance }_{1-3}=\sqrt{\left(x_{1}-x_{3}\right)^{2}+\left(y_{1}-y_{3}\right)^{2}}=\sqrt{(17-96)^{2}+(281-185)^{2}}=124 \mathrm{~m}
$$

Note that the $\mathrm{x}$ and $\mathrm{y}$ coordinates of Node 1 are 17 and 281 respectively.

Then Node 1 arranges its neighboring nodes in the ascending order of their distance, which results in the MPNT of Node 1, as shown in Table 5.4.

Table 5.4: MPNT of Node 1

\begin{tabular}{|c|c|c|}
\hline Node ID & Neighbor Node ID & Distance (m) \\
\hline 1 & 30 & 44 \\
\hline 1 & 7 & 103 \\
\hline 1 & 25 & 122 \\
\hline 1 & 3 & 124 \\
\hline 1 & 32 & 150 \\
\hline 1 & 33 & 152 \\
\hline 1 & 5 & 160 \\
\hline
\end{tabular}

Similarly, each node builds its MPNT, and sends it along with its position and node ID to the gateway node using its control radio over the control channel.

\section{Building the DNT}

For each node in the network, the gateway builds the DNT based on the MPNT of that node and its neighboring nodes. Based on information in the MPNT of Node 1 and the MPNT of its neighboring Node 3, shown in Table 5.5, the gateway does not select 
Node 3 as the neighbor of Node 1 as distance between Node 1 and Node 3 (distance $_{1-3}=$ $124 \mathrm{~m}$ ) is more than the distance between Node 3 and Node 7 (distance $3-7=30 \mathrm{~m}$ ). Node 3 is closer to Node 7 than to Node 1.

Table 5.5: MPNT of Node 3

\begin{tabular}{|c|c|c|}
\hline Node ID & Neighbor Node ID & Distance (m) \\
\hline 3 & 7 & 30 \\
\hline 3 & 33 & 48 \\
\hline 3 & 11 & 66 \\
\hline 3 & 25 & 71 \\
\hline 3 & 32 & 96 \\
\hline 3 & 20 & 120 \\
\hline 3 & 19 & 124 \\
\hline 3 & 1 & 128 \\
\hline 3 & 30 & 144 \\
\hline 3 & 10 & 148 \\
\hline 3 & 34 & 49 \\
\hline
\end{tabular}

Similarly, the gateway removes all such nodes from the MPNT of Node 1 which are closer to any node in their MPNTs than to Node 1 . After removing all such nodes from the MPNT of Node 1, the remaining number of nodes in the MPNT of Node 1 is shown in Table 5.6.

Table 5.6: Remaining nodes in MPNT of Node 1

\begin{tabular}{|c|c|c|}
\hline Node ID & Neighbor Node ID & Distance (m) \\
\hline 1 & 30 & 44 \\
\hline
\end{tabular}

\section{i) DNT for Select 1 for less than 1 TCA}

For Select 1 for less than 1 TCA, since after removing nodes from the MPNT of Node 1 , the remaining number of nodes in MPNT of Node 1 is equal to 1 , the result is the DNT of Node 1, as shown in Table 5.7.

Table 5.7: DNT of Node 1 for Select 1 for less than 1 TCA

\begin{tabular}{|c|c|c|}
\hline Node ID & Neighbor Node ID & Distance $(\mathrm{m})$ \\
\hline 1 & 30 & 44 \\
\hline
\end{tabular}

\section{ii) DNT for Select 2 for less than 2 TCA}

For Select 2 for less than 2 TCA, since after removing nodes from the MPNT of Node 1, the remaining number of nodes in the MPNT of Node 1 is less than 2, the 
gateway selects 2 nearest nodes as neighbors of Node 1, which results in the DNT of Node 1 as shown in Table 5.8.

Table 5.8: DNT of Node 1 for Select 2 for less than 2 TCA

\begin{tabular}{|c|c|c|}
\hline Node ID & Neighbor Node ID & Distance (m) \\
\hline 1 & 30 & 44 \\
\hline 1 & 7 & 103 \\
\hline
\end{tabular}

iii) DNT for Select 3 for less than 3 TCA

For Select 3 for less than 3 TCA, since after removing nodes from the MPNT of Node 1, the remaining number of nodes in the MPNT of Node 1 is less than 3, the gateway selects 3 nearest nodes as neighbors of Node 1, which results in the DNT of Node 1 as shown in Table 5.9 .

Table 5.9: DNT of Node 1 for Select 3 for less than 3 TCA

\begin{tabular}{|c|c|c|}
\hline Node ID & Neighbor Node ID & Distance $(\mathrm{m})$ \\
\hline 1 & 30 & 44 \\
\hline 1 & 7 & 103 \\
\hline 1 & 25 & 122 \\
\hline
\end{tabular}

\section{Converting into Bi-directional Links}

For each node in the network, the gateway converts the uni-directional links in the DNT of a node into bi-directional links. For each uni-directional link, this is done by adding a reverse link in the DNT of the neighboring node. The DNT of Node 7 for Select 3 for less than 3 TCA is shown in Table 5.10.

Table 5.10: DNT of Node 7 for Select 3 for less than 3 TCA

\begin{tabular}{|c|c|c|}
\hline Node ID & Neighbor Node ID & Distance (m) \\
\hline 7 & 3 & 30 \\
\hline 7 & 11 & 75 \\
\hline 7 & 33 & 76 \\
\hline
\end{tabular}

As shown in DNT of Node 1 in Table 5.9, there is a link from Node 1 to Node 7. However, there is no reverse link from Node 7 to Node 1 as shown in the DNT of Node 7 in Table 5.10. So, the gateway adds the link from Node 7 to Node 1 in the DNT of Node 7. This converts the DNT into Bi-directional DNT, which results in the FNT. The FNT of Node 7 for Select 3 for less than 3 TCA is shown in Table 5.11. 
Table 5.11: FNT of Node 7 for Select 3 for less than 3 TCA

\begin{tabular}{|c|c|c|}
\hline Node ID & Neighbor Node ID & Distance (m) \\
\hline 7 & 3 & 30 \\
\hline 7 & 11 & 75 \\
\hline 7 & 33 & 76 \\
\hline 7 & 30 & 101 \\
\hline 7 & 1 & 103 \\
\hline
\end{tabular}

The FNT of Node 1 for Select 3 for less than 3 TCA is shown in Table 5.12.

Table 5.12: FNT of Node 1 for Select 3 for less than 3 TCA

\begin{tabular}{|c|c|c|}
\hline Node ID & Neighbor Node ID & Distance (m) \\
\hline 1 & 30 & 44 \\
\hline 1 & 7 & 103 \\
\hline 1 & 25 & 122 \\
\hline
\end{tabular}

\section{E. Calculating the Minimum Power Required}

For each node in the network, the gateway calculates the minimum power, required to reach each of the nodes in the FNT of a node, using the appropriate propagation model formulas. For example, the cross over distance is calculated by using equation (1) as follows:

$$
\text { Cross_over_dist }=\frac{4 \pi h h_{r}}{\lambda}=\frac{4 \pi \times 3 \times 3}{\left(\frac{3 \times 10^{8}}{5.805 \times 10^{9}}\right)}=2189 \mathrm{~m}
$$

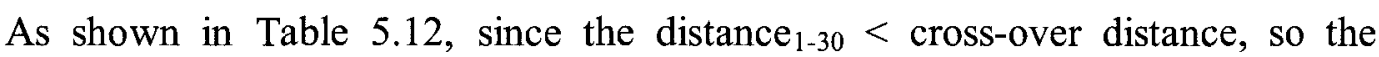
gateway uses the free-space propagation model, given by Equation (2), to calculate the power required to reach Node 30 from Node 1 as follows:

$$
P_{\min , 1-30}=\frac{R x T h r e s h\left(4 \pi d_{1-30}\right)^{2}}{G_{t} G_{r} \lambda^{2}}=\frac{3.16227 \times 10^{-7} \times(4 \pi \times 44)^{2}}{1 \times 1 \times\left(\frac{3 \times 10^{8}}{5.805 \times 10^{9}}\right)^{2}}=37 \mathrm{~mW}
$$

Similarly, the gateway calculates the minimum power required to reach each of the nodes in the FNT of Node 1, as shown in Table 5.13.

Table 5.13: FNT of Node 1 with power required to reach its neighbors

\begin{tabular}{|c|c|c|c|}
\hline Node ID & Neighbor Node ID & Distance (m) & Power (mW) \\
\hline 1 & 30 & 44 & 37 \\
\hline 1 & 7 & 103 & 199 \\
\hline 1 & 25 & 122 & 279 \\
\hline
\end{tabular}




\subsubsection{Connectivity Graph}

Based on the information of the FNTs of all nodes, the gateway builds the connectivity graph and checks the resulting network for connectivity. Note that Node 15 is the gateway node.

\section{A. Connectivity Graph for Select 1 for Less Than 1 TCA}

The FNTs of all nodes resulting from Select 1 for less than 1 TCA are shown in the Appendix in Table A.1. Based on these FNTs, the gateway builds the connectivity graph as shown in Figure 5.5.

Note that the connectivity graph resulting from Select 1 for less than 1 TCA is not connected.

\section{B. Connectivity Graph for Select 2 for Less Than 2 TCA}

Since the connectivity graph resulting from Select 1 for less than 1 is not connected, the gateway moves to the next higher TCA. The FNTs of all nodes resulting from Select 2 for less than 2 TCA are shown in the Appendix in Table A.2. Based on these FNTs, the gateway builds the connectivity graph as shown in Figure 5.6.

Note that the connectivity graph resulting from Select 2 for less than 2 TCA is not connected.

\section{Connectivity Graph for Select 3 for Less Than 3 TCA}

Since the connectivity graph resulting from Select 2 for less than 2 TCA is not connected, the gateway moves to the next higher TCA. The FNTs of all nodes resulting from Select 3 for less than 3 TCA are shown in the Appendix in Table A.3. Based on these FNTs, the gateway builds the connectivity graph as shown in Figure 5.7.

Note that the connectivity graph resulting from Select 3 for less than 3 TCA is connected.

\subsubsection{MPSPT with a MND of Four}

Since the network resulting from Select 3 for less than 3 TCA is connected, the gateway proceeds to build the SPT with a MND of four based on the connectivity graph. The metric for path selection is minimum power to reach the gateway and the tree is rooted at the gateway. The resulting MPSPT with a MND of four is shown in Figure 5.8. 


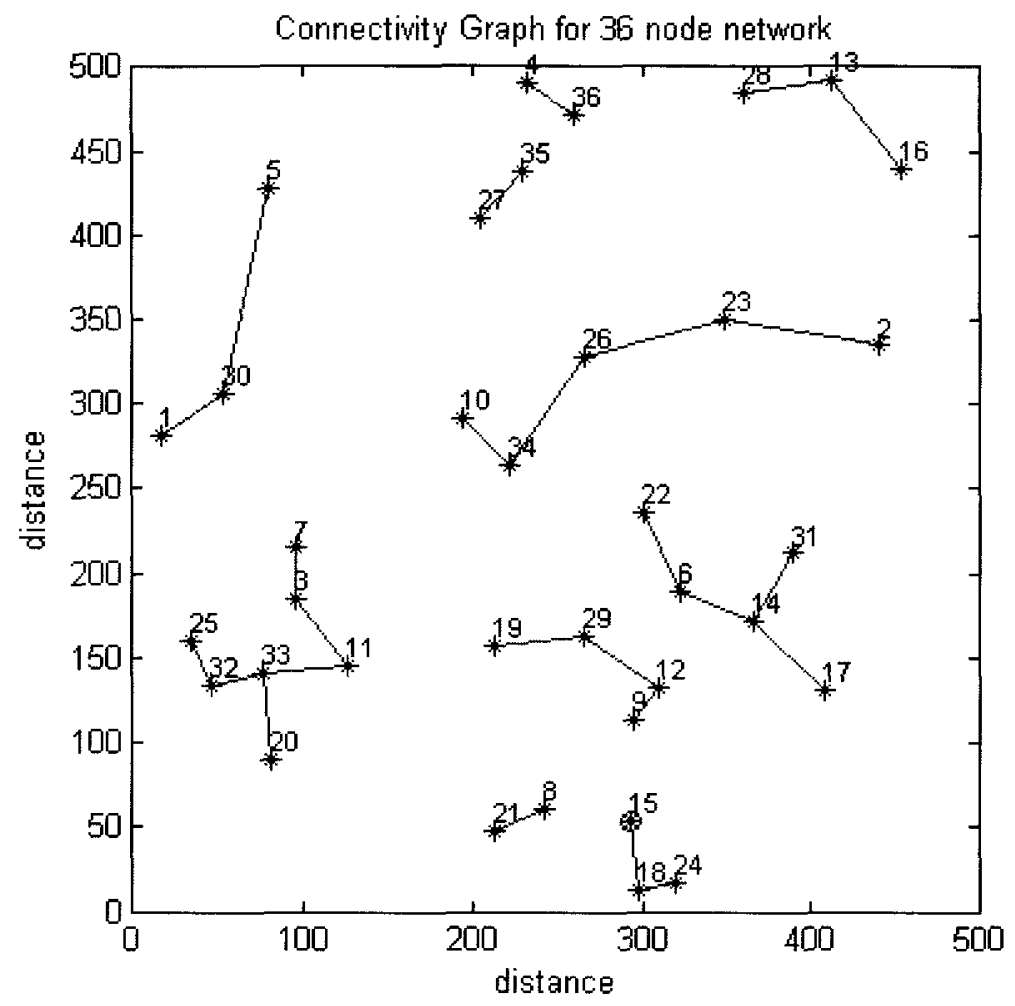

Figure 5.5: Connectivity Graph for Select 1 for less than 1 TCA

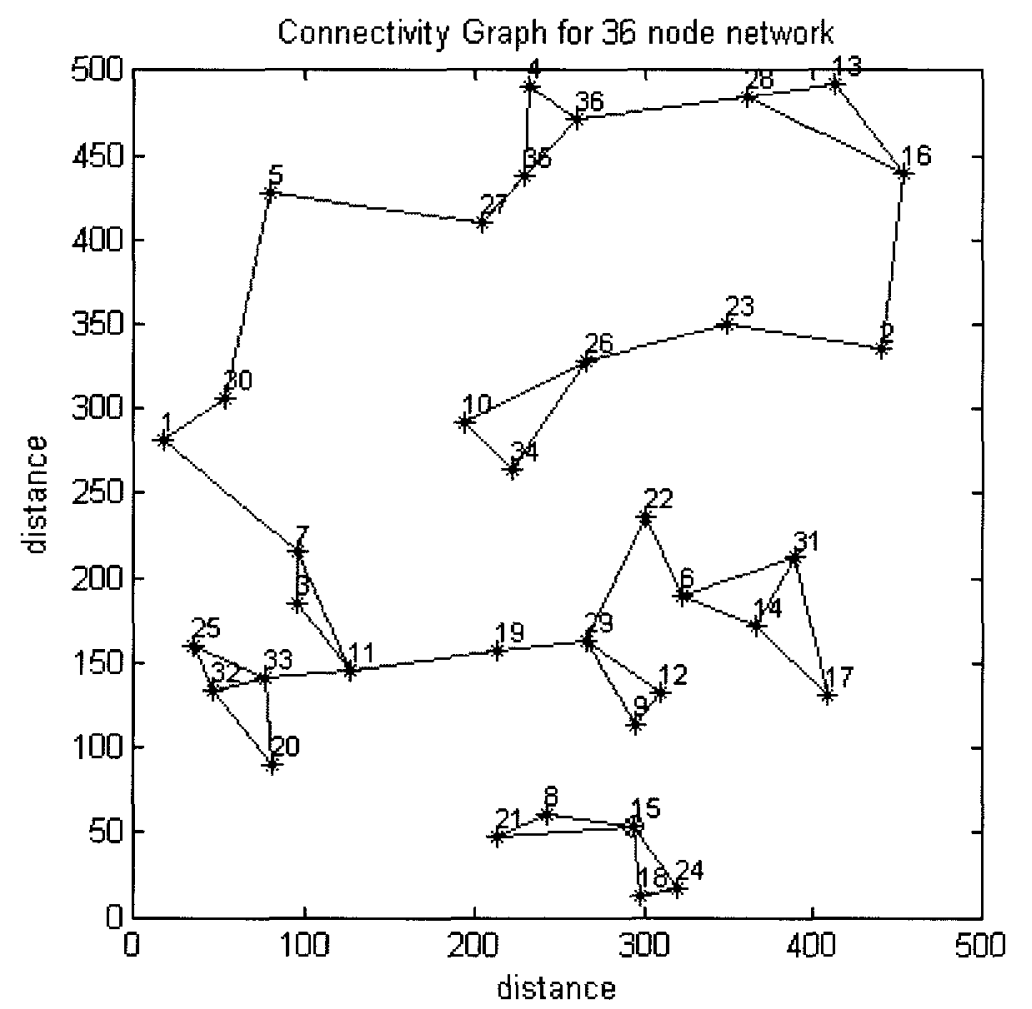

Figure 5.6: Connectivity Graph for Select 2 for less than 2 TCA 


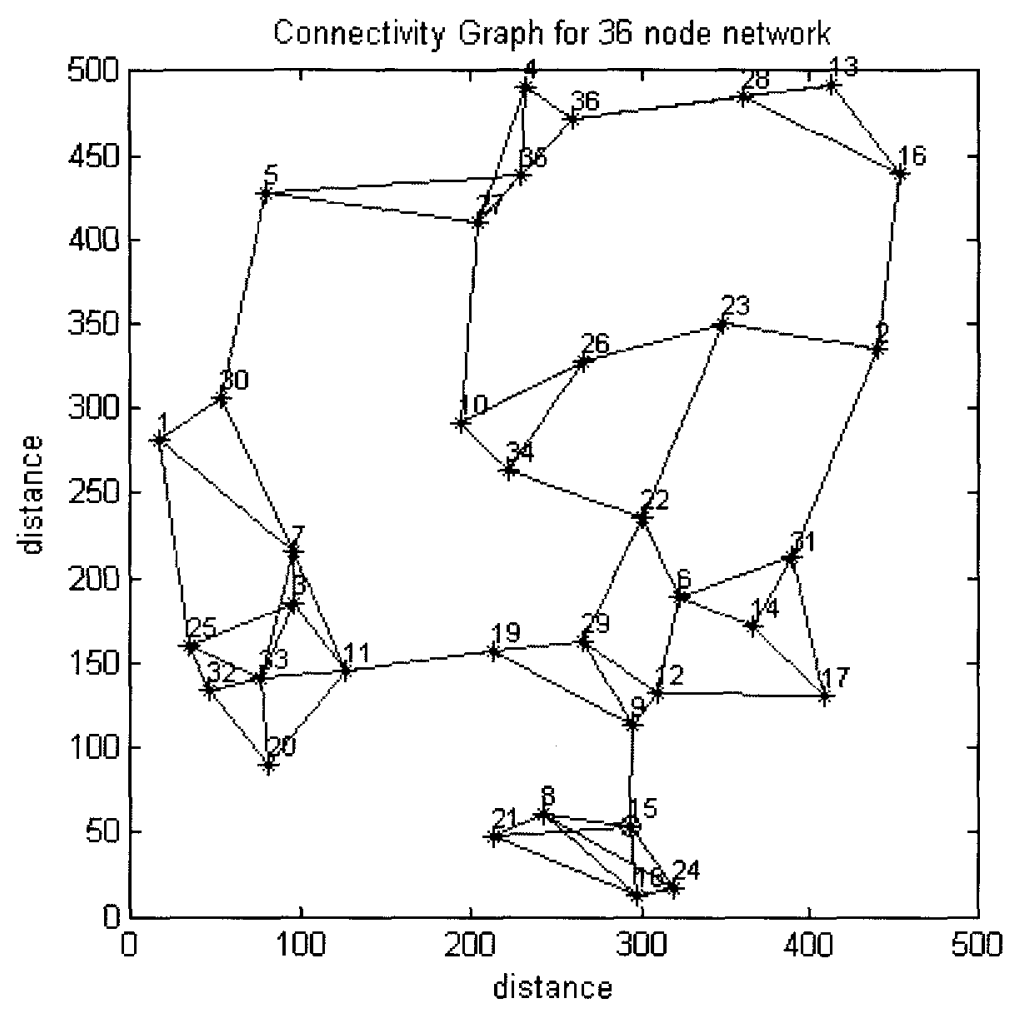

Figure 5.7: Connectivity Graph for Select 3 for less than 3 TCA

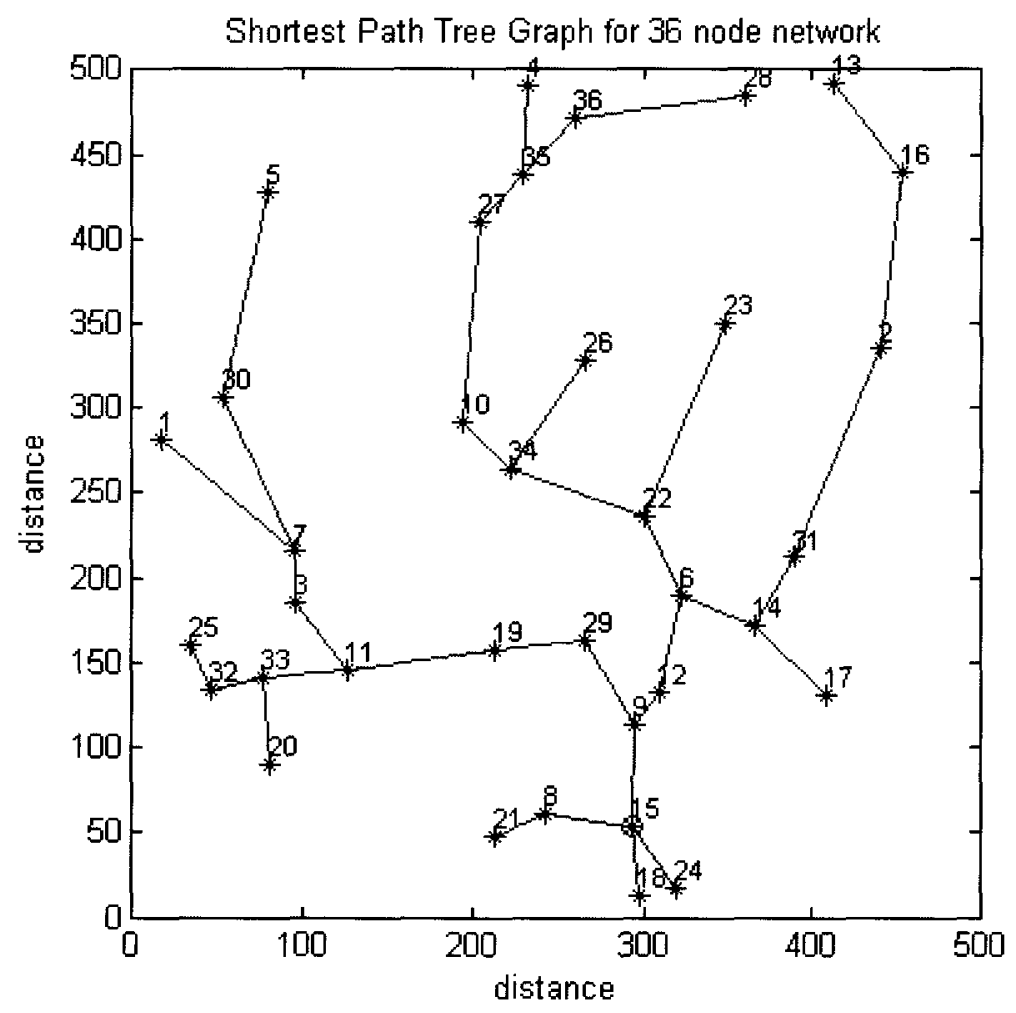

Figure 5.8: MPSP'T with a MND of four for Select 3 for less than 3 TCA 
For each node in the MPSPT, if the gateway finds more than 4 links, it selects four links with minimum weight and sets the weight for all other links to infinity. For example, when the gateway starts to build the MPSPT, it has the following five links, as shown in Table 5.14.

Table 5.14: Possible Links for the gateway

\begin{tabular}{|c|c|c|c|}
\hline Node ID & Neighbor ID & Distance (m) & Power (mW) \\
\hline 15 & 18 & 42 & 33 \\
\hline 15 & 24 & 46 & 40 \\
\hline 15 & 8 & 51 & 49 \\
\hline 15 & 9 & 60 & 68 \\
\hline 15 & 21 & 81 & 123 \\
\hline
\end{tabular}

Out of the five available links, the gateway selects those four links which have the minimum power and sets the weight of the fifth link to Node 21 to infinity, as shown in Table 5.15.

Table 5.15: Links selected by the gateway

\begin{tabular}{|c|c|c|c|}
\hline Node ID & Neighbor ID & Distance (m) & Power (mW) \\
\hline 15 & 18 & 42 & 33 \\
\hline 15 & 24 & 46 & 40 \\
\hline 15 & 8 & 51 & 49 \\
\hline 15 & 9 & 60 & 68 \\
\hline 15 & 21 & 81 & INF \\
\hline
\end{tabular}

Similarly, the gateway checks each node for a maximum of four links and builds a MPSPT with a MND of four per node.

\subsubsection{Link Ranking}

Since the resulting MPSPT is connected, the gateway proceeds to assign a ranking to each link of the MPSPT. The ranking associated with each link is derived from the number of nodes that use a link to reach the gateway. For example, the rank of link between Node 15 and Node 9 is calculated, using equation (4), as follows:

$$
r_{15-9}=\sum_{n=1}^{N} I_{n, l}=\sum_{n=1}^{36} I_{n, 15-9}=I_{1,15-9}+I_{2,15-9}+\ldots+I_{36,15-9}=31
$$

Similarly, the gateway calculates the rank of all links in the MPSPT. If two or more links have the same rank, then the link whose power of the farthest node to the gateway is smallest is given priority. If there are still some links with the same rank, then 
the link with the smallest node IDs is given priority. The resulting link ranking for the MPSPT is shown in Table 5.16.

Table 5.16: Link Ranking of MPSPT

\begin{tabular}{|c|c|c|c|c|c|}
\hline \multicolumn{2}{|c|}{ Link } & $\begin{array}{l}\text { Distance } \\
(\mathrm{m})\end{array}$ & $\begin{array}{l}\text { Power } \\
\text { (mW) }\end{array}$ & RANK & $\begin{array}{l}\text { Power from the farthest node of } \\
\text { the link to the gateway (mW) }\end{array}$ \\
\hline 15 & 9 & 60 & 68 & 31 & 68 \\
\hline 9 & 12 & 24 & 11 & 18 & 79 \\
\hline 12 & 6 & 58 & 63 & 17 & 142 \\
\hline 9 & 29 & 57 & 61 & 12 & 129 \\
\hline 29 & 19 & 53 & 53 & 11 & 182 \\
\hline 6 & 22 & 52 & 51 & 10 & 193 \\
\hline 19 & 11 & 88 & 145 & 10 & 327 \\
\hline 22 & 34 & 84 & 132 & 8 & 325 \\
\hline 6 & 14 & 46 & 40 & 6 & 182 \\
\hline 34 & 10 & 40 & 30 & 6 & 355 \\
\hline 11 & 3 & 49 & 45 & 5 & 372 \\
\hline 10 & 27 & 119 & 265 & 5 & 620 \\
\hline 14 & 31 & 47 & 42 & 4 & 224 \\
\hline 11 & 33 & 49 & 45 & 4 & 372 \\
\hline 3 & 7 & 30 & 17 & 4 & 389 \\
\hline 27 & 35 & 38 & 27 & 4 & 647 \\
\hline 31 & 2 & 133 & 331 & 3 & 555 \\
\hline 15 & 8 & 51 & 49 & 2 & 49 \\
\hline 33 & 32 & 32 & 20 & 2 & 392 \\
\hline 7 & 30 & 101 & 191 & 2 & 580 \\
\hline 35 & 36 & 46 & 40 & 2 & 687 \\
\hline 2 & 16 & 106 & 211 & 2 & 766 \\
\hline 15 & 18 & 42 & 33 & 1 & 33 \\
\hline 15 & 24 & 46 & 40 & 1 & 40 \\
\hline 8 & 21 & 33 & 21 & 1 & 70 \\
\hline 14 & 17 & 59 & 66 & 1 & 248 \\
\hline 32 & 25 & 28 & 15 & 1 & 407 \\
\hline 33 & 20 & 51 & 49 & 1 & 421 \\
\hline 34 & 26 & 78 & 114 & 1 & 439 \\
\hline 22 & 23 & 124 & 288 & 1 & 481 \\
\hline 7 & 1 & 103 & 199 & 1 & 588 \\
\hline 35 & 4 & 53 & 53 & 1 & 700 \\
\hline 16 & 13 & 66 & 82 & 1 & 848 \\
\hline 30 & 5 & 125 & 293 & 1 & 873 \\
\hline 36 & 28 & 101 & 191 & 1 & 878 \\
\hline
\end{tabular}

As shown in Table 5.16, the link between Node 6 and Node 22 and link between

Node 19 and Node 11 have the same rank. In this case, the link between 6 and 22 is given 
priority on the link between 19 and 11 as the power from Node 22 to the gateway is $193 \mathrm{~mW}$ which is smaller than the power from Node 11 to the gateway which is $327 \mathrm{~mW}$.

\subsubsection{Channel Assignment}

The gateway assigns a channel to each link in the order of its rank, first assigning the 11 available non-overlapping channels to the 11 highest-ranked links as shown in Table 5.17.

Table 5.17: Channel Assignment of the 11 highest-ranked links

\begin{tabular}{|c|c|c|}
\hline \multicolumn{2}{|c|}{ Link } & Channel \\
\hline 15 & 9 & 1 \\
\hline 9 & 12 & 2 \\
\hline 12 & 6 & 3 \\
\hline 9 & 29 & 4 \\
\hline 29 & 19 & 5 \\
\hline 6 & 22 & 6 \\
\hline 19 & 11 & 7 \\
\hline 22 & 34 & 8 \\
\hline 6 & 14 & 9 \\
\hline 34 & 10 & 10 \\
\hline 11 & 3 & 11 \\
\hline
\end{tabular}

For the $12^{\text {th }}$-ranked link and onwards, the gateway checks the channel assignment of all links within the interference range of both nodes that constitute that link. For example, for the $12^{\text {th }}$-ranked link between Node 10 and Node 27 , the gateway finds all the channel-assigned links within the interference range of Node 10 and Node 27, as shown in Table 5.18 and Figures 5.9 and 5.10 respectively.

Since the gateway cannot find any channel among the 11 available channels that are not assigned to any link within the IR of both nodes that constitute the $12^{\text {th }}$-ranked link, it computes a LIC and assigns it to the link between Node 10 and Node 27. 


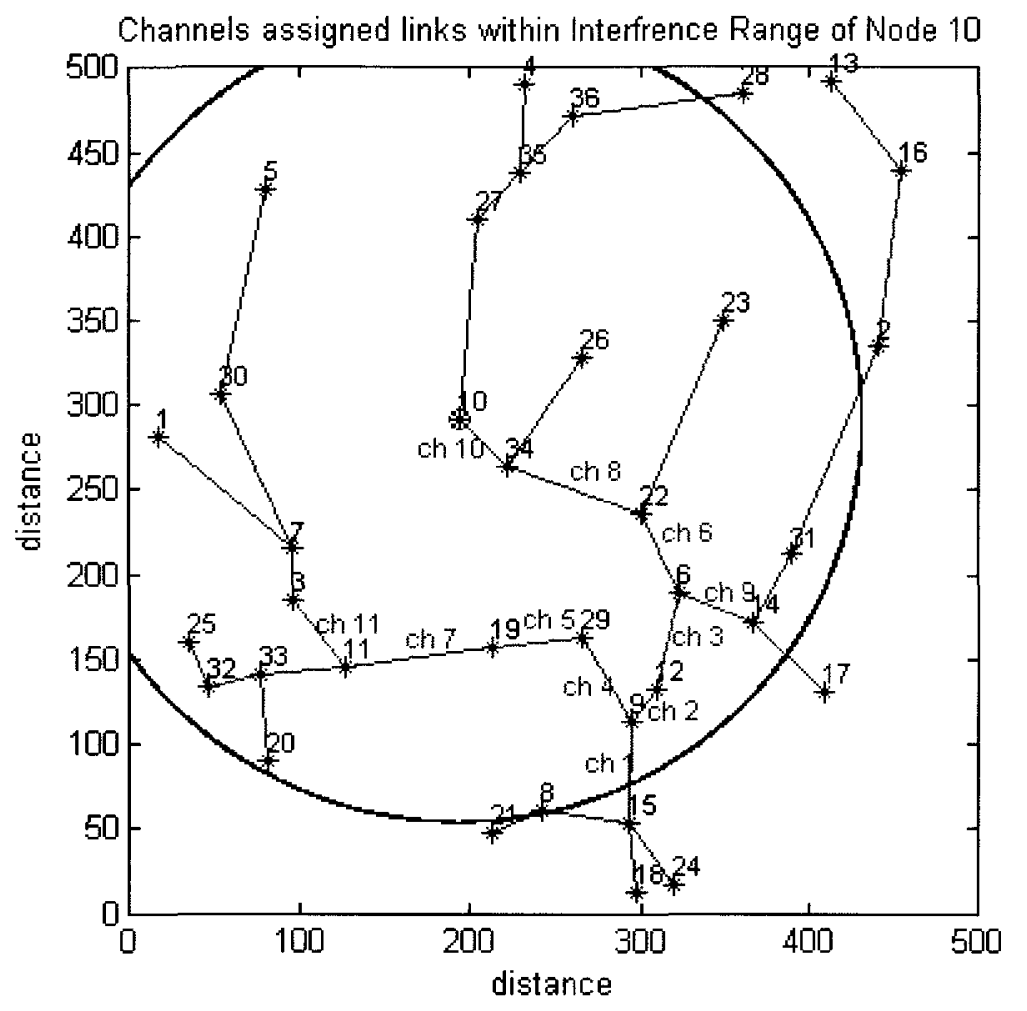

Figure 5.9: Channel-assigned links within IR of Node 10

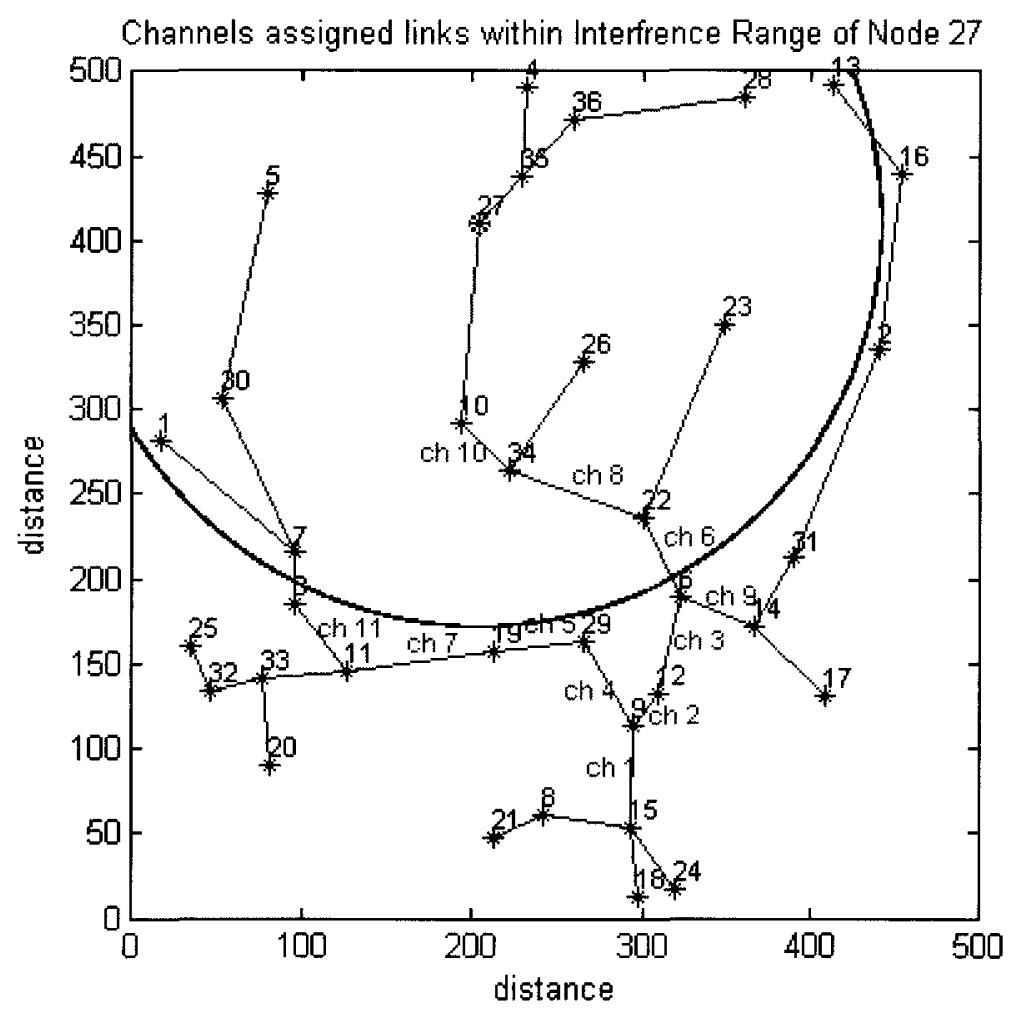

Figure 5.10: Channel-assigned links within IR of Node 27 
Table 5.18: Channel Assignment of links within IR of $12^{\text {th }}$-ranked link

\begin{tabular}{|c|c|c|c|c|c|}
\hline Node ID & \multicolumn{2}{|c|}{ Link } & Channel & Distance & RANK \\
\hline 10 & 10 & 34 & 10 & 0 & 6 \\
\hline 10 & 3 & 11 & 11 & 144 & 5 \\
\hline 10 & 6 & 12 & 3 & 166 & 17 \\
\hline 10 & 6 & 22 & 6 & 166 & 10 \\
\hline 10 & 6 & 14 & 9 & 166 & 6 \\
\hline 10 & 9 & 15 & 1 & 205 & 31 \\
\hline 10 & 9 & 12 & 2 & 205 & 18 \\
\hline 10 & 9 & 29 & 4 & 205 & 12 \\
\hline 10 & 11 & 19 & 7 & 161 & 10 \\
\hline 10 & 11 & 3 & 11 & 161 & 5 \\
\hline 10 & 12 & 9 & 2 & 197 & 18 \\
\hline 10 & 12 & 6 & 3 & 197 & 17 \\
\hline 10 & 14 & 6 & 9 & 211 & 6 \\
\hline 10 & 19 & 29 & 5 & 136 & 11 \\
\hline 10 & 19 & 11 & 7 & 136 & 10 \\
\hline 10 & 22 & 6 & 6 & 121 & 10 \\
\hline 10 & 22 & 34 & 8 & 121 & 8 \\
\hline 10 & 29 & 9 & 4 & 148 & 12 \\
\hline 10 & 29 & 19 & 5 & 148 & 11 \\
\hline 10 & 34 & 22 & 8 & 40 & 8 \\
\hline 10 & 34 & 10 & 10 & 40 & 6 \\
\hline 27 & 10 & 34 & 10 & 119 & 6 \\
\hline 27 & 22 & 6 & 6 & 199 & 10 \\
\hline 27 & 22 & 34 & 8 & 199 & 8 \\
\hline 27 & 34 & 22 & 8 & 147 & 8 \\
\hline 27 & 34 & 10 & 10 & 147 & 6 \\
\hline
\end{tabular}

\section{A. Interference Level}

In order to find out the LIC, the gateway calculates the interference level for all the 11 channels. For example, the gateway calculates the $\mathrm{IL}$ for channel nine, using equation (5) as follows:

$$
(I L)_{9}=\sum_{m}\left(\frac{r_{m}}{R}\right)\left(\frac{1}{d_{m}^{\alpha}}\right)=\left(\frac{6}{31}\right)\left(\frac{1}{166^{2}}\right)+\left(\frac{6}{31}\right)\left(\frac{1}{211^{2}}\right)=1.137117 \times 10^{-5}
$$

Similarly, the gateway calculates the IL for all the 11 channels as shown in Table 5.19. Note that since another link is emanating from Node 10 , i.e. link between Node 10 and Node 34, and channel 10 has been assigned to this link, therefore, the IL for channel 10 is set to infinity. 
Table 5.19: Interference Level for all 11 channels

\begin{tabular}{|c|c|}
\hline Channel & IL \\
\hline 1 & $2.379536 \mathrm{e}-05$ \\
\hline 2 & $2.877827 \mathrm{e}-05$ \\
\hline 3 & $3.403123 \mathrm{e}-05$ \\
\hline 4 & $2.688353 \mathrm{e}-05$ \\
\hline 5 & $3.538434 \mathrm{e}-05$ \\
\hline 6 & $4.188483 \mathrm{e}-05$ \\
\hline 7 & $2.988532 \mathrm{e}-05$ \\
\hline 8 & $1.973755 \mathrm{e}-04$ \\
\hline 9 & $1.137117 \mathrm{e}-05$ \\
\hline 10 & INF \\
\hline 11 & $1.400066 \mathrm{e}-05$ \\
\hline
\end{tabular}

\section{B. Least Interfering Channel}

As shown in Table 5.19, channel 9 is the channel with the minimum IL. So, using equation (6) as follows, the gateway selects channel 9 as the LIC and assigns it to the $12^{\text {th }}$-ranked link.

$$
\begin{gathered}
(I L)_{L I C}=\min \left[(I L)_{1},(I L)_{2}, \ldots \ldots \ldots,(I L)_{11}\right] \\
(I L)_{L I C}=(I L)_{9} \\
\Rightarrow L I C=9
\end{gathered}
$$

\section{Non-conflicting Channel}

For the $13^{\text {th }}$-ranked link between Nodes 14 and 31 , the gateway finds Channels 5 , 7,10 , and 11, which are non-conflicting channels as they are not assigned to any links within IR range of both nodes that constitute the $13^{\text {th }}$-ranked link, as shown in Table 5.20.

From the available non-conflicting channels, the gateway selects the channel with the highest channel number, i.e. Channel 11 and assigns it to the $13^{\text {th }}$-ranked link. Similarly, the gateway assigns channels to all links in the MPSPT, as shown in Table 5.21 . 
Table 5.20: Channel Assignment of links within IR of $13^{\text {th }}$-ranked link

\begin{tabular}{|c|c|c|c|c|c|}
\hline Node ID & \multicolumn{2}{|c|}{ Link } & Channel & Distance & RANK \\
\hline 14 & 14 & 6 & 9 & 0 & 6 \\
\hline 14 & 6 & 12 & 3 & 46 & 17 \\
\hline 14 & 6 & 22 & 6 & 46 & 10 \\
\hline 14 & 6 & 14 & 9 & 46 & 6 \\
\hline 14 & 9 & 15 & 1 & 92 & 31 \\
\hline 14 & 9 & 12 & 2 & 92 & 18 \\
\hline 14 & 9 & 29 & 4 & 92 & 12 \\
\hline 14 & 12 & 9 & 2 & 69 & 18 \\
\hline 14 & 12 & 6 & 3 & 69 & 17 \\
\hline 14 & 22 & 6 & 6 & 92 & 10 \\
\hline 14 & 22 & 34 & 8 & 92 & 8 \\
\hline 31 & 6 & 12 & 3 & 71 & 17 \\
\hline 31 & 6 & 22 & 6 & 71 & 10 \\
\hline 31 & 6 & 14 & 9 & 71 & 6 \\
\hline 31 & 14 & 6 & 9 & 47 & 6 \\
\hline 31 & 22 & 6 & 6 & 93 & 10 \\
\hline 31 & 22 & 34 & 8 & 93 & 8 \\
\hline
\end{tabular}

Table 5.21: Channel Assignment for links of MPSPT

\begin{tabular}{|c|c|c||c|c|c|}
\hline \multicolumn{2}{|c|}{ Link } & \multicolumn{2}{c|}{ Channel } & \multicolumn{2}{c|}{ Channel } \\
\hline 15 & 9 & 1 & 33 & 32 & 8 \\
\hline 9 & 12 & 2 & 7 & 30 & 6 \\
\hline 12 & 6 & 3 & 35 & 36 & 10 \\
\hline 9 & 29 & 4 & 2 & 16 & 5 \\
\hline 29 & 19 & 5 & 15 & 18 & 10 \\
\hline 6 & 22 & 6 & 15 & 24 & 9 \\
\hline 19 & 11 & 7 & 8 & 21 & 8 \\
\hline 22 & 34 & 8 & 14 & 17 & 10 \\
\hline 6 & 14 & 9 & 32 & 25 & 11 \\
\hline 34 & 10 & 10 & 33 & 20 & 5 \\
\hline 11 & 3 & 11 & 34 & 26 & 2 \\
\hline 10 & 27 & 9 & 22 & 23 & 7 \\
\hline 14 & 31 & 11 & 7 & 1 & 3 \\
\hline 11 & 33 & 10 & 35 & 4 & 8 \\
\hline 3 & 7 & 9 & 16 & 13 & 11 \\
\hline 27 & 35 & 2 & 30 & 5 & 4 \\
\hline 31 & 8 & 7 & 36 & 28 & 6 \\
\hline 15 & & 11 & & \\
\hline
\end{tabular}

\section{Channel Assignment and Routing Message}

The gateway then sends each mesh node the CARM, via the control radio on the control channel, which contains the channel number and the neighbor node to 
communicate with using this channel. The channel assignment message also contains the next hop to reach the gateway for data traffic. Based on channel assigned to a mesh router to communicate with a neighbor and its distance to that neighbor, the mesh router applies power control and adjusts its transmission power by using the appropriate propagation model based on equation (1).

\subsection{Failure Recovery Mechanism of TICA}

All nodes send periodic "keep-alive" messages to the gateway. If the gateway does not receive three consecutive keep-alives from any node, it concludes that that node has failed and is no longer active. After deleting the MPNT for this node and deleting it from the MPNT of all its neighboring nodes, the gateway builds the FNTs for all nodes. The gateway then proceeds with all the phases required to calculate the channel assignment.

After calculating the new channel assignment, the gateway sends the new CARM to all nodes in the network on the control channel.

In order to understand the different phases of FRM of TICA, the random topology shown in Figure 5.2 is considered and four different scenarios of failure recovery with TICA are discussed.

\subsubsection{Scenario 1 - Node 9 Fails}

In this scenario, I suppose that Node 9 has failed. After missing three consecutive keep-alives from Node 9, the gateway concludes that Node 9 is not active. The gateway then deletes the MPNT of Node 9 and deletes Node 9 from the MPNT of its neighbors. The gateway runs the Select 3 for less than 3 TCA, builds the FNTs for all nodes and builds the connectivity graph as shown in Figure 5.11. The gateway starts with Select 3 for less than 3 TCA, as it resulted in a connected network when Node 9 was alive. Note that the network resulting from Select 3 for less than 3 TCA is not connected.

Since the connectivity graph resulting from Select 3 for less than 3 TCA is not connected, the gateway moves to Select 4 for less than 4 TCA and builds the connectivity graph as shown in Figure 5.12. Note that the network resulting from Select 4 for less than 4 TCA is connected.

Since the connectivity graph resulting from Select 4 for less than 4 TCA is connected, the gateway builds the MPSPT with a MND of 4, as shown in Figure 5.13. 


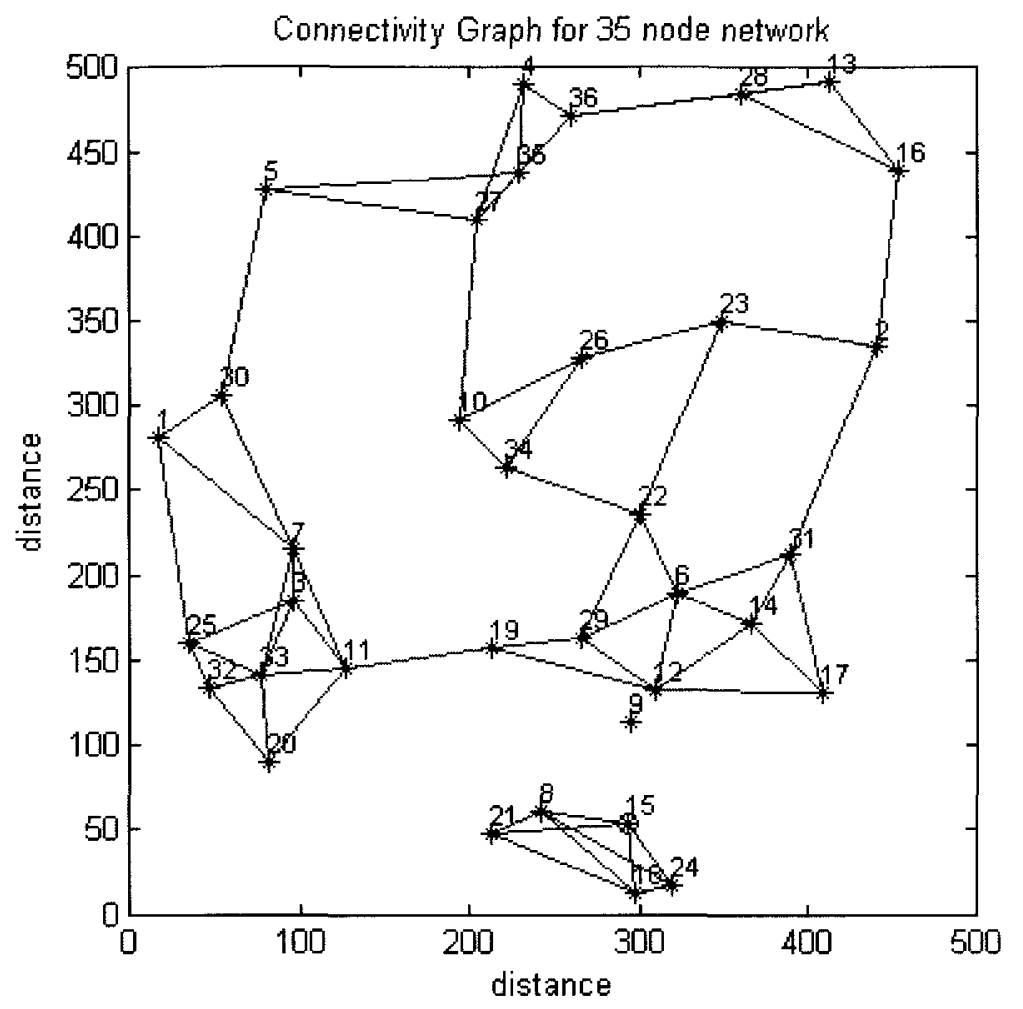

Figure 5.11: Connectivity Graph with Select 3 for less than 3 TCA after Node 9 fails

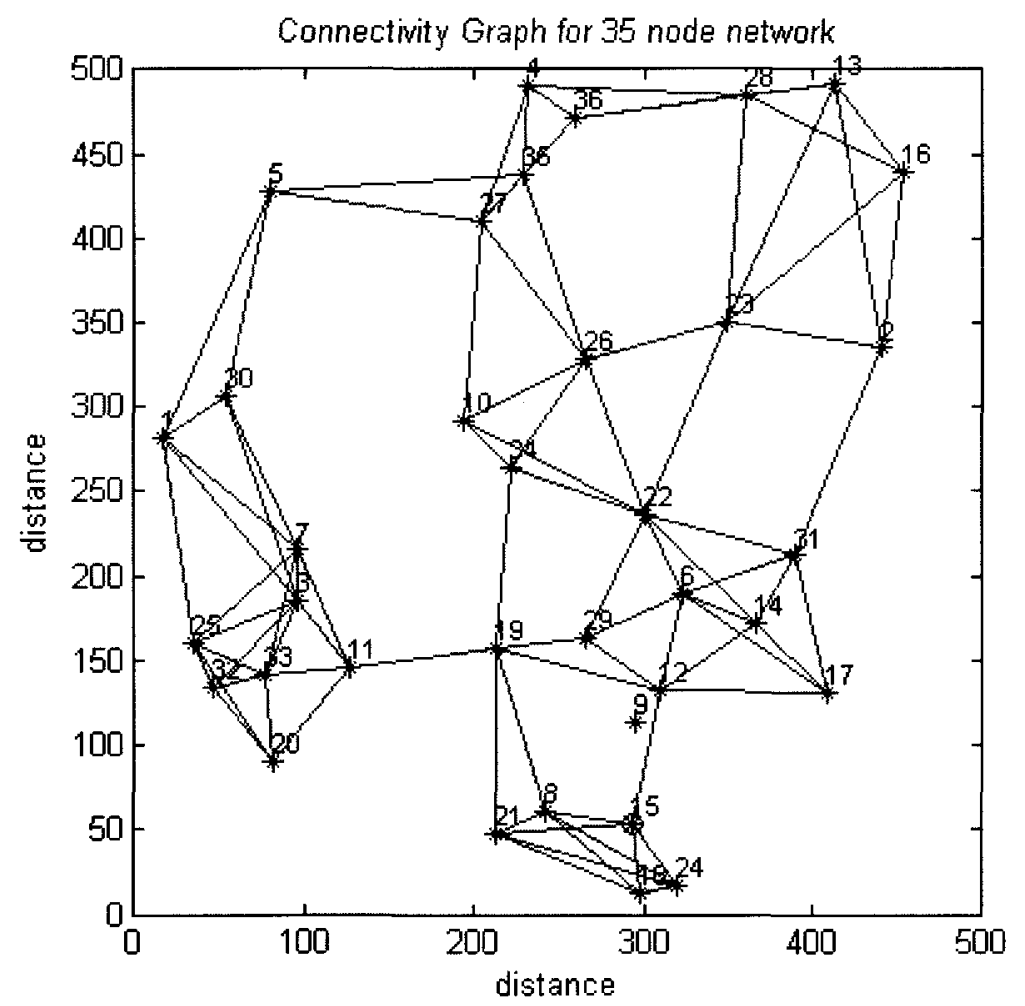

Figure 5.12: Connectivity Graph with Select 4 for less than 4 TCA after Node 9 fails 


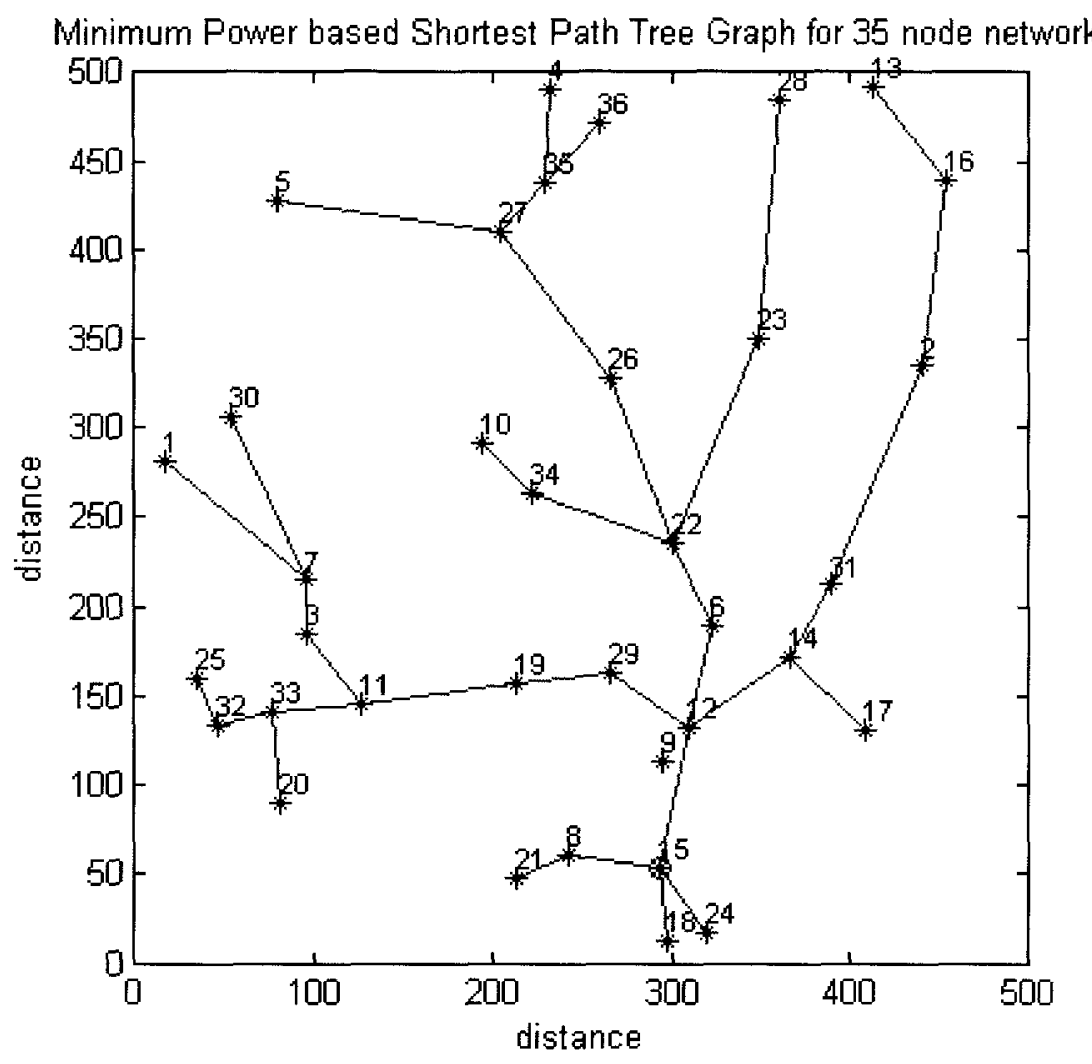

Figure 5.13: MPSPT with a MND of 4 after Node 9 fails

Since the resulting MPSPT is connected, the gateway builds the link ranking for each link of the MPSPT, as shown in Table 5.22.

Based on the link ranking, the gateway assigns a channel to each link in the MPSPT, as shown in Table 5.23.

Node 9 was part of the highest ranked link before its failure. So, after its failure, the link rankings and in turn, the channel assignment of all links in the MPSPT changes.

Note that the other three scenarios for failure recovery with TICA are presented in the appendix $B$.

During failure recovery with TICA, after deleting the MPNT of the failed node and deleting the failed node from the MPNTs of its neighbors, the gateway runs the TCA and builds the MPSPT. Based on the new MPSPT, the link rankings and hence, the channel assignments of links change. 
Table 5.22: Link Ranking of MPSPT after Node 9 fails

\begin{tabular}{|c|c|c|c|c|c|}
\hline \multicolumn{2}{|c|}{ Link } & Distance & Power & RANK & Power from the farthest node of \\
\hline 15 & 12 & 81 & 123 & 30 & 123 \\
\hline 12 & 6 & 58 & 63 & 12 & 186 \\
\hline 12 & 29 & 52 & 51 & 11 & 174 \\
\hline 6 & 22 & 52 & 51 & 11 & 237 \\
\hline 29 & 19 & 53 & 53 & 10 & 227 \\
\hline 19 & 11 & 88 & 145 & 9 & 372 \\
\hline 12 & 14 & 69 & 90 & 6 & 213 \\
\hline 22 & 26 & 98 & 180 & 6 & 417 \\
\hline 26 & 27 & 103 & 199 & 5 & 616 \\
\hline 14 & 31 & 47 & 42 & 4 & 255 \\
\hline 11 & 3 & 49 & 45 & 4 & 417 \\
\hline 11 & 33 & 49 & 45 & 4 & 417 \\
\hline 3 & 7 & 30 & 17 & 3 & 434 \\
\hline 31 & 2 & 133 & 331 & 3 & 586 \\
\hline 27 & 35 & 38 & 27 & 3 & 643 \\
\hline 15 & 8 & 51 & 49 & 2 & 49 \\
\hline 22 & 34 & 84 & 132 & 2 & 369 \\
\hline 33 & 32 & 32 & 20 & 2 & 437 \\
\hline 22 & 23 & 124 & 288 & 2 & 525 \\
\hline 2 & 16 & 106 & 211 & 2 & 797 \\
\hline 15 & 18 & 42 & 33 & 1 & 33 \\
\hline 15 & 24 & 46 & 40 & 1 & 40 \\
\hline 8 & 21 & 33 & 21 & 1 & 70 \\
\hline 14 & 17 & 59 & 66 & 1 & 279 \\
\hline 34 & 10 & 40 & 30 & 1 & 399 \\
\hline 32 & 25 & 28 & 15 & 1 & 452 \\
\hline 33 & 20 & 51 & 49 & 1 & 466 \\
\hline 7 & 30 & 101 & 191 & 1 & 625 \\
\hline 7 & 1 & 103 & 199 & 1 & 633 \\
\hline 35 & 36 & 46 & 40 & 1 & 683 \\
\hline 35 & 4 & 53 & 53 & 1 & 696 \\
\hline 23 & 28 & 136 & 346 & 1 & 871 \\
\hline 16 & 13 & 66 & 82 & 1 & 879 \\
\hline 27 & 5 & 126 & 297 & 1 & 913 \\
\hline
\end{tabular}


Table 5.23: Channel Assignment for links of MPSPT after Node 9 fails

\begin{tabular}{|c|c|c|}
\hline Node & Neighbor & Channel \\
\hline 15 & 12 & 1 \\
\hline 12 & 6 & 2 \\
\hline 12 & 29 & 3 \\
\hline 6 & 22 & 4 \\
\hline 29 & 19 & 5 \\
\hline 19 & 11 & 6 \\
\hline 12 & 14 & 7 \\
\hline 22 & 26 & 8 \\
\hline 26 & 27 & 9 \\
\hline 14 & 31 & 10 \\
\hline 11 & 3 & 11 \\
\hline 11 & 33 & 10 \\
\hline 3 & 7 & 9 \\
\hline 31 & 2 & 11 \\
\hline 27 & 35 & 11 \\
\hline 15 & 8 & 11 \\
\hline 22 & 34 & 11 \\
\hline 33 & 32 & 8 \\
\hline 22 & 23 & 6 \\
\hline 2 & 16 & 5 \\
\hline 15 & 18 & 10 \\
\hline 15 & 24 & 9 \\
\hline 8 & 21 & 8 \\
\hline 14 & 17 & 9 \\
\hline 34 & 10 & 10 \\
\hline 32 & 25 & 11 \\
\hline 33 & 20 & 7 \\
\hline 7 & 30 & 4 \\
\hline 7 & 1 & 2 \\
\hline 35 & 36 & 10 \\
\hline 35 & 4 & 8 \\
\hline 23 & 28 & 7 \\
\hline 16 & 13 & 10 \\
\hline 27 & 5 & 3 \\
\hline
\end{tabular}




\subsection{Node Degree}

The node degree is defined as the number of transmission range neighbors of a node.

\subsubsection{Average Node Degree}

The Average Node Degree (AND) for the entire network can be calculated by equation (7):

$$
A N D=\frac{\sum_{l}^{N} q_{j}}{N}
$$

where $q$ is the number of TR neighbors of a node, and $N$ is the total number of nodes in the network.

\subsubsection{Maximum Power Average Node Degree}

\section{A. Maximum Power TR Average Node Degree}

It is defined as the average number of TR neighbors when all nodes transmit with the same maximum power.

\section{B. Maximum Power IR Average Node Degree}

It is defined as the average number of IR neighbors when all nodes transmit with the same maximum power.

\subsubsection{Minimum Power Average Node Degree}

\section{A. Minimum Power TR Average Node Degree}

It is defined as the average number of TR neighbors resulting from the Select $x$ for less than $x$ TCA.

\section{B. Minimum Power IR Average Node Degree}

It is defined as the average number of IR neighbors resulting from the Select $x$ for less than $x$ TCA.

A high average node degree indicates a stronger connectivity but results in a higher interference in the network. On the other hand, interference will be less in the case of a lower node degree but it will also affect the connectivity of the network and may result in a disconnected network. 


\section{CHAPTER 6}

\section{RESULTS}

In this chapter, the results for the proposed TCA, Select $x$ for less than $x$, and different phases of the proposed CAA, TICA, which include topology control, connectivity graph, MPSPT with the required maximum node degree, link ranking and channel assignment, are presented. The detailed results of simulations for performance evaluation of TICA based on throughput analysis of a 36-node network and a 144-node network are given, and analyzed. The chapter also presents the results for the performance comparison of TICA with well known centralized channel assignment schemes. The features' comparison of TICA with related well known channel assignment schemes is discussed.

\subsection{Topology Control}

\subsubsection{Maximum Power Average Node Degree}

The maximum power transmission range average node degrees for a 36-node network for the three topologies (random, controlled random and grid) are shown in Table 6.1.

Table 6.1: Max. Power TR Average Node Degree

\begin{tabular}{|c|c|c|}
\hline \multicolumn{3}{|c|}{ TOPOLOGY } \\
\hline Random & Controlled Random & Grid \\
\hline 9.33 & 7.5 & 6.11 \\
\hline
\end{tabular}

Table 6.2 shows the maximum power interference range average node degrees for the three topologies.

Table 6.2: Max. Power IR Average Node Degree

\begin{tabular}{|c|c|c|}
\hline \multicolumn{3}{|c|}{ TOPOLOGY } \\
\hline Random & Controlled Random & Grid \\
\hline 17.7 & 16.4 & 17.9 \\
\hline
\end{tabular}




\subsubsection{Minimum Power Average Node Degree}

A comparison of the different topology control algorithms based on the minimum power TR average node degrees for a network of 36 nodes is shown in Table 6.3.

Table 6.3: Min. Power TR Average Node Degree

\begin{tabular}{|c|c|c|c|}
\hline \multirow{2}{*}{ Topology Control Algorithm } & \multicolumn{3}{|c|}{ TOPOLOGY } \\
\cline { 2 - 4 } & $\mathbf{R}$ & $\mathbf{C R}$ & $\mathbf{G}$ \\
\hline Select 1 for less than 1 & 1.44 & 1.61 & 3.33 \\
\hline Select 2 for less than 2 & 2.56 & 2.42 & 3.33 \\
\hline Select 3 for less than 3 & 3.67 & 3.58 & 3.56 \\
\hline Select 4 for less than 4 & 5.06 & 4.75 & 4.39 \\
\hline
\end{tabular}

Figure 8.1 shows a graphical comparison of the different topology control algorithms for the three topologies (random, controlled random and grid) based on the minimum power TR average node degrees for a network of 36 nodes.

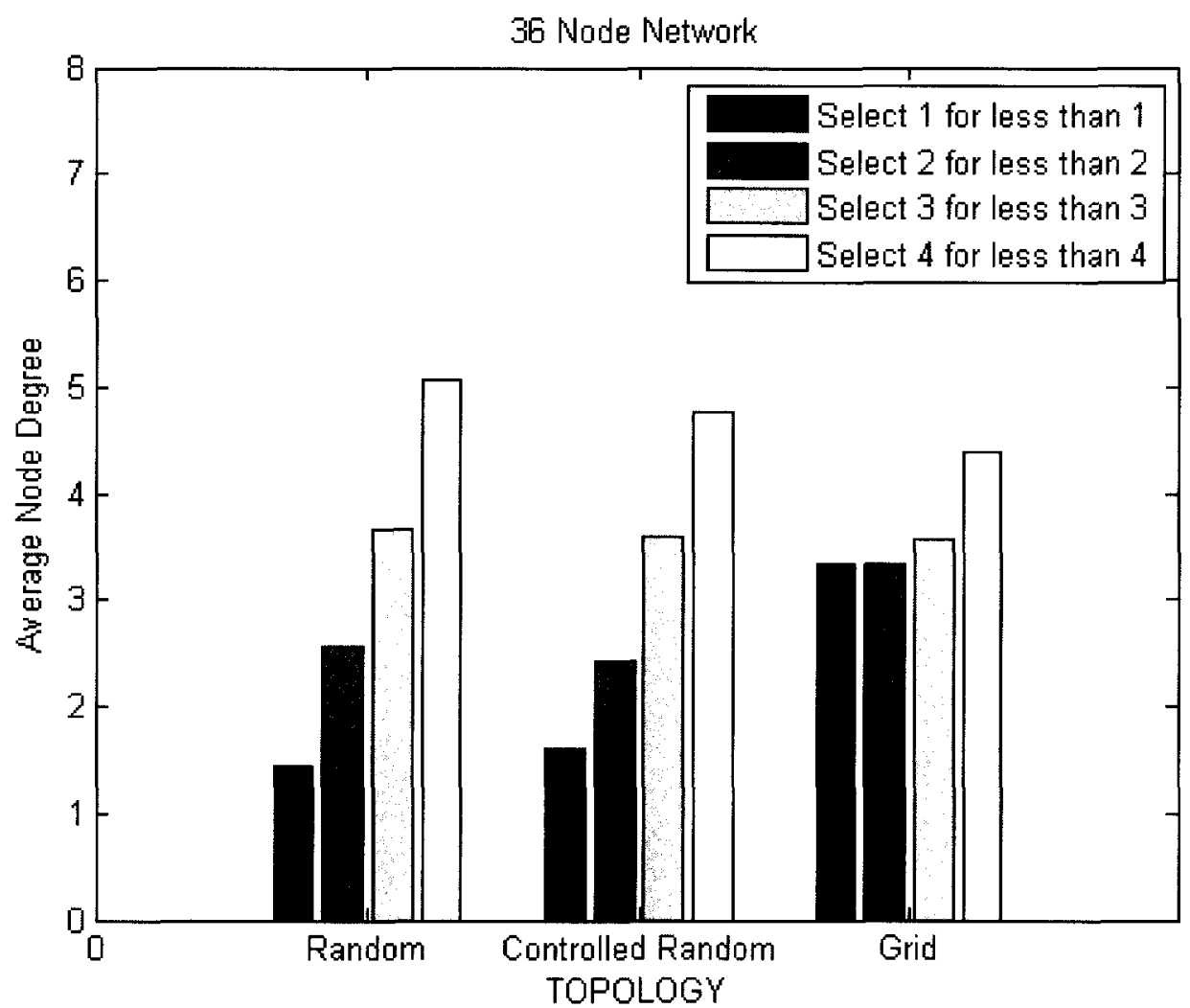

Figure 6.1: Minimum Power TR Average Node Degree

Figure 6.1 shows that as the number of selected neighbors increases from Select 1 for less than 1 TCA to Select 4 for less than 4 TCA, the minimum power TR average node degree increases, which means that the network connectivity increases. 
Table 6.4 shows a comparison of the different topology control algorithms based on the minimum power IR average node degrees.

Table 6.4: Min. Power IR Average Node Degree

\begin{tabular}{|c|c|c|c|}
\hline \multirow{2}{*}{ Topology Control Algorithm } & \multicolumn{3}{|c|}{ TOPOLOGY } \\
\cline { 2 - 4 } & $\mathbf{R}$ & CR & G \\
\hline Select 1 for less than 1 & 3.58 & 4.86 & 2.78 \\
\hline Select 2 for less than 2 & 6.42 & 6.06 & 2.78 \\
\hline Select 3 for less than 3 & 9.28 & 7.78 & 3.56 \\
\hline Select 4 for less than 4 & 10.7 & 9.47 & 7.56 \\
\hline
\end{tabular}

Figure 6.2 shows a graphical comparison of the different topology control algorithms for the three topologies (random, controlled random and grid) based on the minimum power IR average node degrees for a network of 36 nodes.

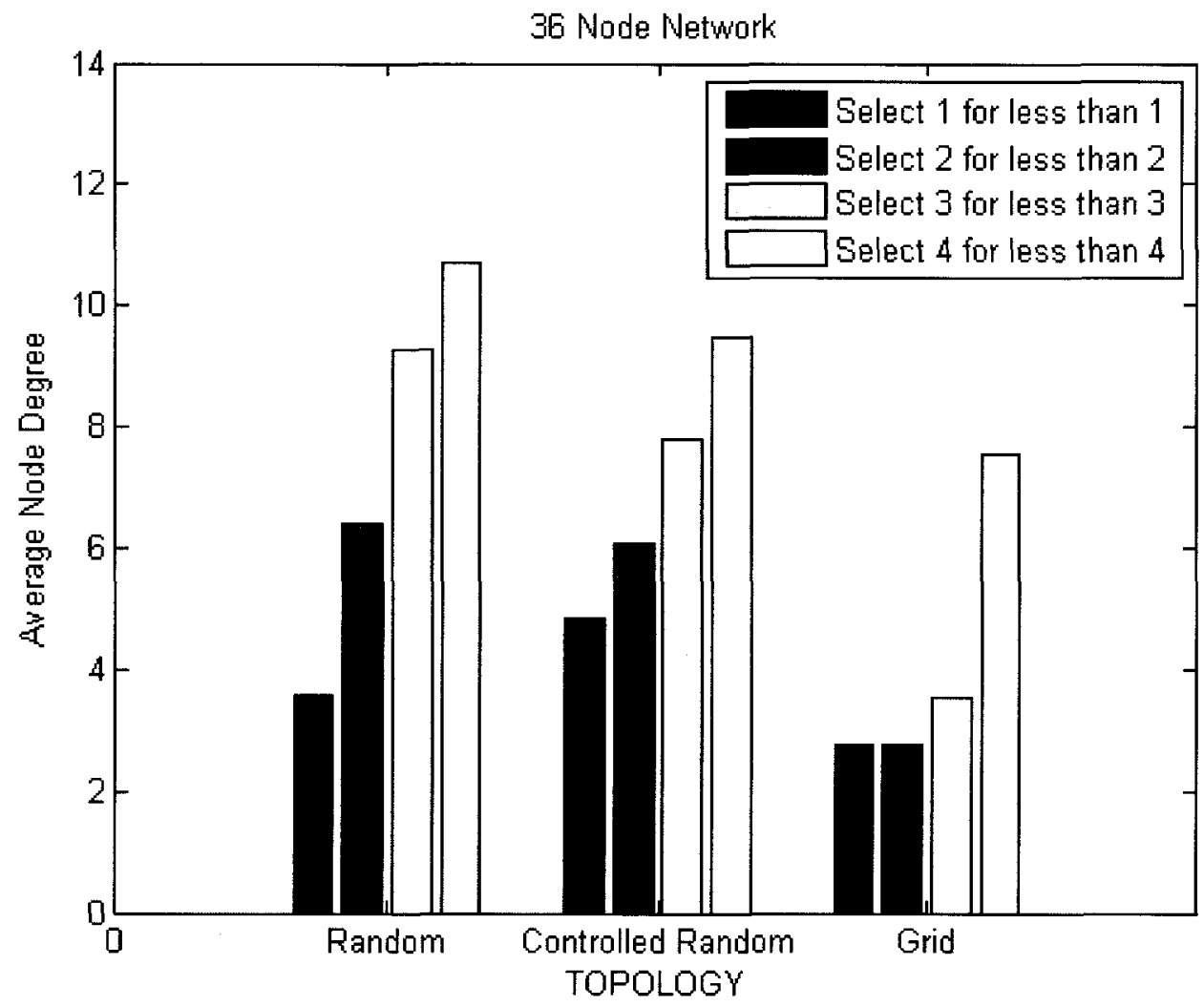

Figure 6.2: Minimum Power IR Average Node Degree

Figure 6.2 shows that as the number of selected neighbors increases from Select 1 for less than 1 TCA to Select 4 for less than 4 TCA, the minimum power IR average node degree increases, which means that the interference in the network increases. 


\subsubsection{Connectivity and Interference}

Table 6.5 indicates whether the connectivity graph resulting from a certain TCA is connected. Note that after receiving the FNTs from the nodes, the gateway builds the connectivity graph.

Table 6.5: Check for connected topology

\begin{tabular}{|c|c|c|c|}
\hline \multirow{2}{*}{ Topology Control Algorithm } & \multicolumn{3}{|c|}{ Connected Topology } \\
\cline { 2 - 4 } & $\mathbf{R}$ & CR & G \\
\hline Select 1 for less than $\mathbf{1}$ & No & No & Yes \\
\hline Select 2 for less than $\mathbf{2}$ & No & No & Yes \\
\hline Select 3 for less than 3 & Yes & Yes & Yes \\
\hline Select 4 for less than 4 & Yes & Yes & Yes \\
\hline
\end{tabular}

Table 6.6 shows the number of disconnected nodes (nodes not reachable by the gateway) in the connectivity graph resulting from a certain TCA.

Table 6.6: Number of disconnected nodes

\begin{tabular}{|c|c|c|c|}
\hline \multirow{2}{*}{ Topology Control Algorithm } & \multicolumn{3}{|c|}{ TOPOLOGY } \\
\cline { 2 - 4 } & R & CR & G \\
\hline Select 1 for less than 1 & 33 & 32 & 0 \\
\hline Select 2 for less than 2 & 31 & 8 & 0 \\
\hline Select 3 for less than 3 & 0 & 0 & 0 \\
\hline Select 4 for less than 4 & 0 & 0 & 0 \\
\hline
\end{tabular}

For random and controlled random topologies, a high minimum power average node degree, in case of Select 3 for less than 3 and Select 4 for less than 4 TCAs, indicates a stronger connectivity and leads to a connected network but results in a higher interference in the network. On the other hand, a lower minimum power average node degree translates to less interference with Select 1 for less than 1 and Select 2 for less than 2 TCAs, but it also affects the connectivity of the network and results in a disconnected network. 


\subsection{Connectivity Graph}

Note that Node 15 is taken as the gateway node for all topologies.

\section{A. Random Topology}

Figure 5.7 shows the connectivity graph for random topology resulting from "Select 3 for less than 3" TCA for a 36-node network. Note that the resulting topology is connected.

\section{B. Controlled Random Topology}

Figure 6.3 shows the connectivity graph for controlled random topology resulting from "Select 3 for less than 3" TCA for a 36-node network. Note that the resulting topology is connected.

\section{Grid Topology}

Figure 6.4 shows the connectivity graph for grid topology resulting from "Select 1 for less than 1" TCA for a 36-node network. Note that the resulting topology is connected.

\subsection{Minimum Power-based Shortest Path Tree}

\section{A. Random Topology}

Figure 5.8 shows the MPSPT graph for random topology resulting from "Select 3 for less than 3" TCA for a 36-node network with a MND of 4.

\section{B. Controlled Random Topology}

Figure 6.5 shows the MPSPT graph for controlled random topology resulting from "Select 3 for less than 3" TCA for a 36-node network with a MND of 4.

\section{Grid Topology}

Figure 6.6 shows the MPSPT graph for grid topology resulting from "Select 1 for less than 1" TCA for a 36-node network with a maximum node degree of 4 . 


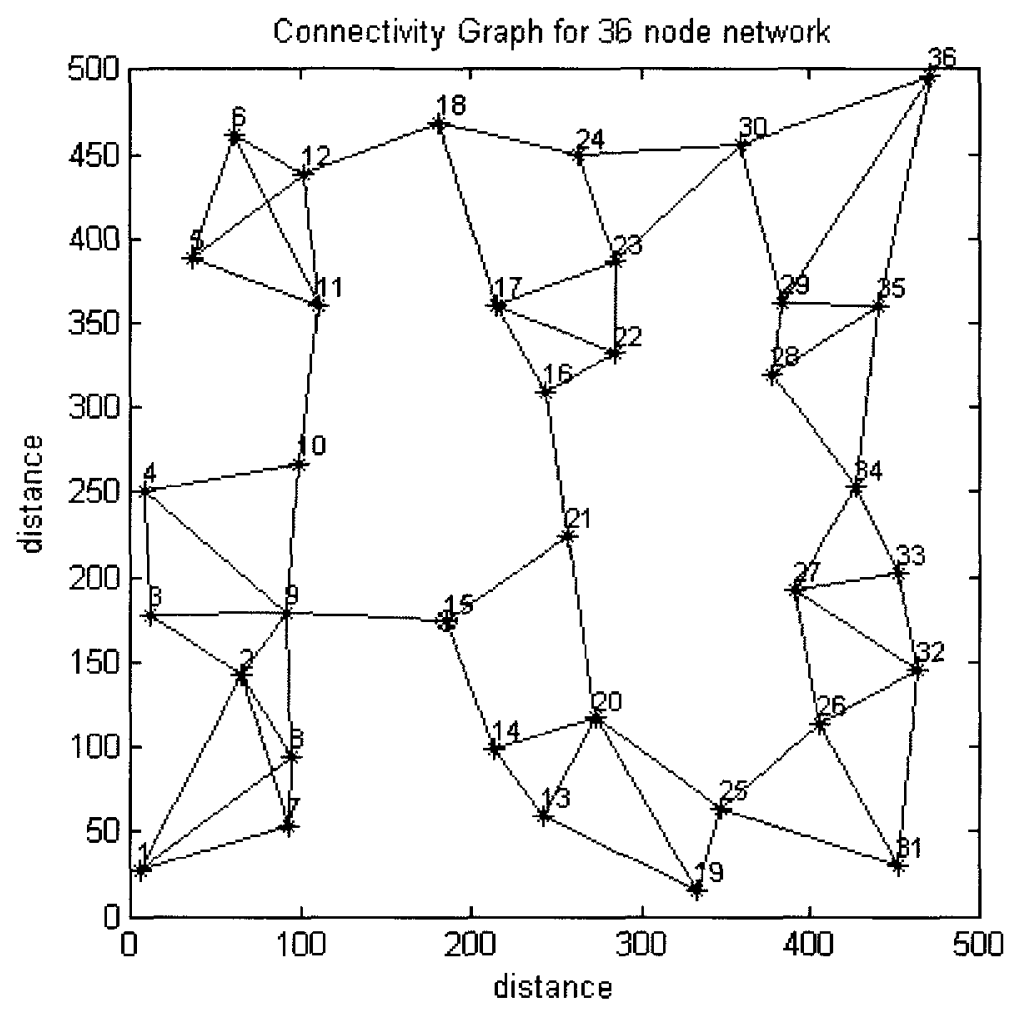

Figure 6.3: Connectivity Graph (CRT)

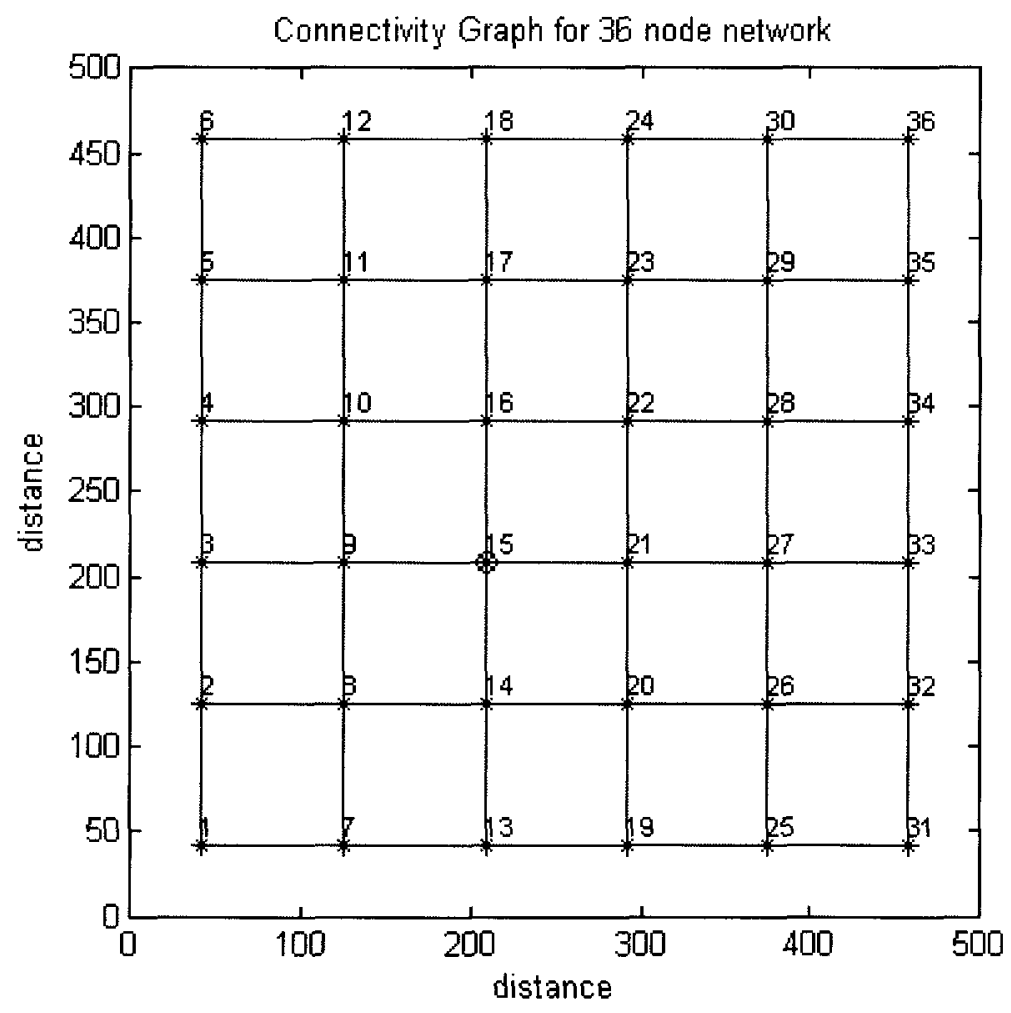

Figure 6.4: Connectivity Graph (GT) 


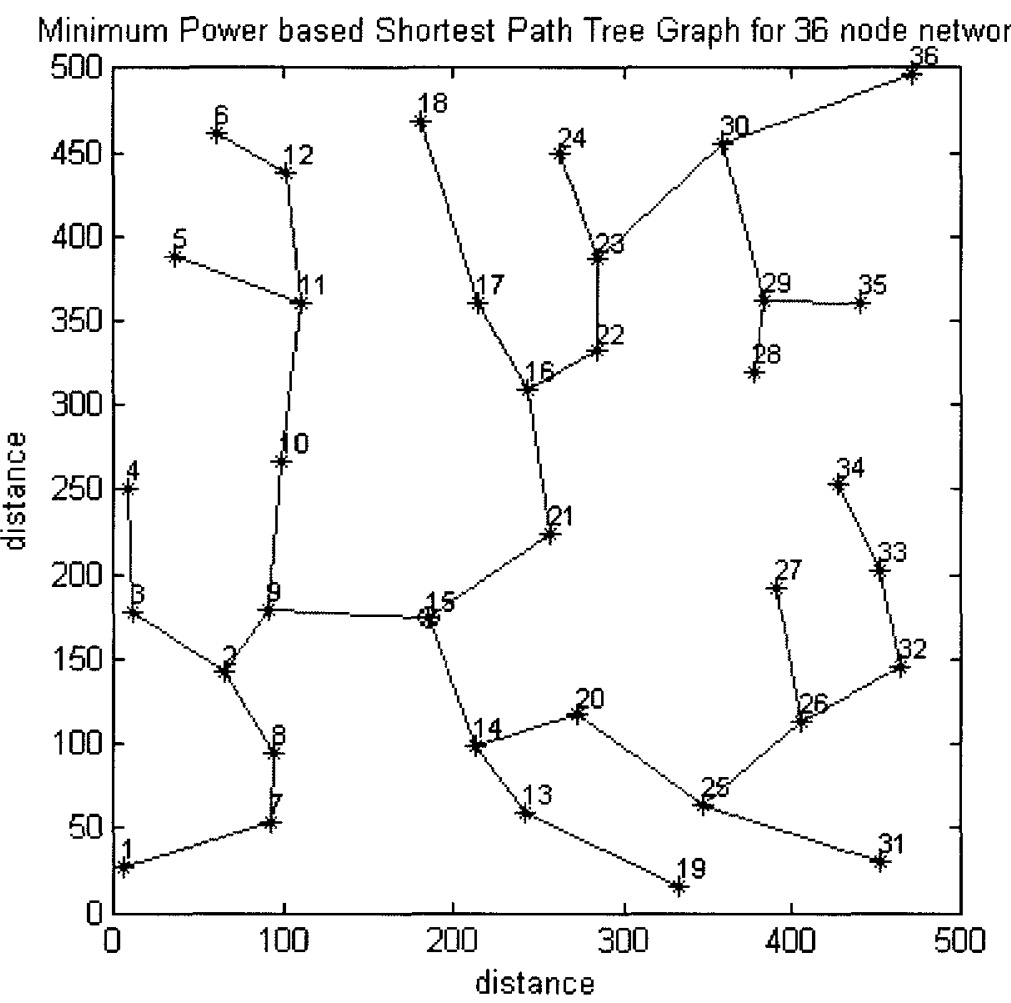

Figure 6.5: MPSPT Graph (CRT)

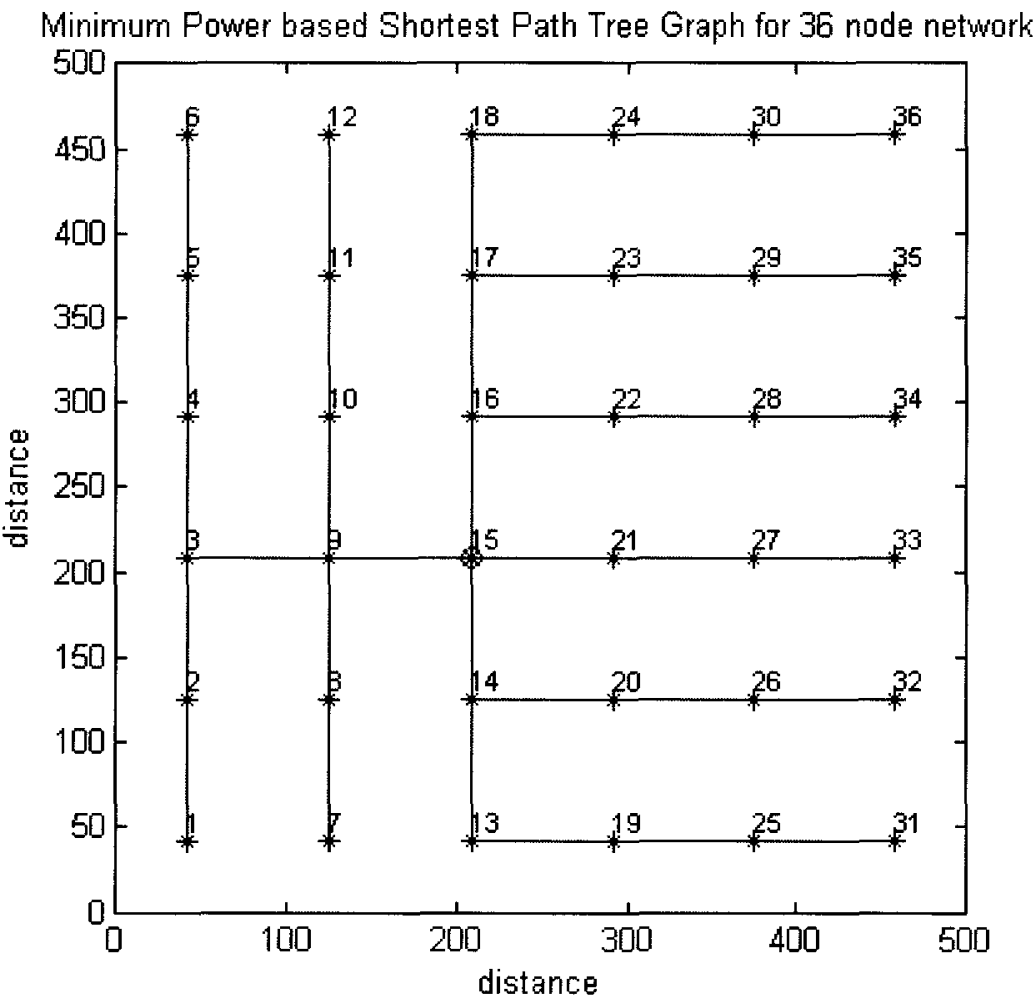

Figure 6.6: MPSPT Graph (GT) 
6.4. Link Ranking

\section{A. Random Topology}

Table 5.16 shows link ranking of MPSPT graph for random topology.

\section{B. Controlled Random Topology}

Table 6.7 shows link ranking of MPSPT graph for controlled random topology.

Table 6.7: Link Ranking of MPSPT (CRT)

\begin{tabular}{|c|c|c|c|c|c|}
\hline \multicolumn{2}{|c|}{ Link } & $\begin{array}{l}\text { Distance } \\
\text { (m) }\end{array}$ & $\begin{array}{l}\text { Power } \\
\text { (mw) }\end{array}$ & RANK & Power from the farthest node of \\
\hline 15 & 21 & 88 & 145 & 12 & 145 \\
\hline 15 & 9 & 93 & 162 & 12 & 162 \\
\hline 15 & 14 & 80 & 120 & 11 & 120 \\
\hline 21 & 16 & 86 & 139 & 11 & 284 \\
\hline 14 & 20 & 62 & 72 & 8 & 192 \\
\hline 16 & 22 & 47 & 42 & 8 & 326 \\
\hline 20 & 25 & 92 & 159 & 7 & 351 \\
\hline 22 & 23 & 54 & 55 & 7 & 381 \\
\hline 9 & 2 & 46 & 40 & 6 & 202 \\
\hline 9 & 10 & 88 & 145 & 5 & 307 \\
\hline 25 & 26 & 77 & 111 & 5 & 462 \\
\hline 23 & 30 & 102 & 195 & 5 & 576 \\
\hline 10 & 11 & 94 & 166 & 4 & 473 \\
\hline 2 & 8 & 55 & 57 & 3 & 259 \\
\hline 26 & 32 & 67 & 84 & 3 & 546 \\
\hline 30 & 29 & 96 & 173 & 3 & 749 \\
\hline 14 & 13 & 49 & 45 & 2 & 165 \\
\hline 2 & 3 & 65 & 79 & 2 & 281 \\
\hline 8 & 7 & 42 & 33 & 2 & 292 \\
\hline 16 & 17 & 59 & 66 & 2 & 350 \\
\hline 11 & 12 & 78 & 114 & 2 & 587 \\
\hline 32 & 33 & 58 & 63 & 2 & 609 \\
\hline 13 & 19 & 101 & 191 & 1 & 356 \\
\hline 3 & 4 & 73 & 100 & 1 & 381 \\
\hline 7 & 1 & 92 & 159 & 1 & 451 \\
\hline 23 & 24 & 66 & 82 & 1 & 463 \\
\hline 25 & 31 & 110 & 227 & 1 & 578 \\
\hline 26 & 27 & 80 & 120 & 1 & 582 \\
\hline 11 & 5 & 79 & 117 & 1 & 590 \\
\hline 17 & 18 & 114 & 243 & 1 & 593 \\
\hline 12 & 6 & 47 & 42 & 1 & 629 \\
\hline 33 & 34 & 56 & 59 & 1 & 668 \\
\hline 29 & 28 & 42 & 33 & 1 & 782 \\
\hline 29 & 35 & 57 & 61 & 1 & 810 \\
\hline 30 & 36 & 121 & 274 & 1 & 850 \\
\hline
\end{tabular}




\section{Grid Topology}

Table 6.8 shows link ranking of MPSPT graph for grid topology.

Table 6.8: Link Ranking of MPSPT (GT)

\begin{tabular}{|c|c|c|c|c|c|}
\hline \multicolumn{2}{|c|}{ Link } & $\begin{array}{c}\text { Distance } \\
(m)\end{array}$ & $\begin{array}{l}\text { Power } \\
\text { (mW) }\end{array}$ & RANK & $\begin{array}{l}\text { Power from the farthest node of } \\
\text { the link to the gateway (mW) }\end{array}$ \\
\hline 15 & 9 & 83 & 129 & 12 & 129 \\
\hline 15 & 16 & 83 & 129 & 12 & 129 \\
\hline 15 & 14 & 83 & 129 & 8 & 129 \\
\hline 16 & 17 & 83 & 129 & 8 & 258 \\
\hline 9 & 3 & 83 & 129 & 6 & 258 \\
\hline 14 & 13 & 83 & 129 & 4 & 258 \\
\hline 17 & 18 & 83 & 129 & 4 & 387 \\
\hline 15 & 21 & 83 & 129 & 3 & 129 \\
\hline 9 & 10 & 83 & 129 & 3 & 258 \\
\hline 14 & 20 & 83 & 129 & 3 & 258 \\
\hline 16 & 22 & 83 & 129 & 3 & 258 \\
\hline 3 & 4 & 83 & 129 & 3 & 387 \\
\hline 13 & 19 & 83 & 129 & 3 & 387 \\
\hline 17 & 23 & 83 & 129 & 3 & 387 \\
\hline 18 & 24 & 83 & 129 & 3 & 516 \\
\hline 9 & 8 & 83 & 129 & 2 & 258 \\
\hline 21 & 27 & 83 & 129 & 2 & 258 \\
\hline 3 & 2 & 83 & 129 & 2 & 387 \\
\hline 10 & 11 & 83 & 129 & 2 & 387 \\
\hline 20 & 26 & 83 & 129 & 2 & 387 \\
\hline 22 & 28 & 83 & 129 & 2 & 387 \\
\hline 4 & 5 & 83 & 129 & 2 & 516 \\
\hline 19 & 25 & 83 & 129 & 2 & 516 \\
\hline 23 & 29 & 83 & 129 & 2 & 516 \\
\hline 24 & 30 & 83 & 129 & 2 & 645 \\
\hline 8 & 7 & 83 & 129 & 1 & 387 \\
\hline 27 & 33 & 83 & 129 & 1 & 387 \\
\hline 2 & 1 & 83 & 129 & 1 & 516 \\
\hline 11 & 12 & 83 & 129 & 1 & 516 \\
\hline 26 & 32 & 83 & 129 & 1 & 516 \\
\hline 28 & 34 & 83 & 129 & 1 & 516 \\
\hline 5 & 6 & 83 & 129 & 1 & 645 \\
\hline 25 & 31 & 83 & 129 & 1 & 645 \\
\hline 29 & 35 & 83 & 129 & 1 & 645 \\
\hline 30 & 36 & 83 & 129 & 1 & 774 \\
\hline
\end{tabular}




\subsection{Channel Assignment}

\section{A. Random Topology}

Table 5.21 shows CA for links of MPSPT graph for random topology.

\section{B. Controlled Random Topology}

Table 6.9 shows CA for links of MPSPT graph for controlled random topology.

Table 6.9: Channel Assignment for links of MPSPT (CRT)

\begin{tabular}{|c|c|c|}
\hline \multicolumn{2}{|c|}{ Link } & \multirow{2}{*}{$\begin{array}{c}\text { Channel } \\
1\end{array}$} \\
\hline 15 & 21 & \\
\hline 15 & 9 & 2 \\
\hline 15 & 14 & 3 \\
\hline 21 & 16 & 4 \\
\hline 14 & 20 & 5 \\
\hline 16 & 22 & 6 \\
\hline 20 & 25 & 7 \\
\hline 22 & 23 & 8 \\
\hline 9 & 2 & 9 \\
\hline 9 & 10 & 10 \\
\hline 25 & 26 & 11 \\
\hline 23 & 30 & 11 \\
\hline 10 & 11 & 7 \\
\hline 2 & 8 & 11 \\
\hline 26 & 32 & 10 \\
\hline 30 & 29 & 10 \\
\hline 14 & 13 & 11 \\
\hline 2 & 3 & 8 \\
\hline 8 & 7 & 7 \\
\hline 16 & 17 & 10 \\
\hline 11 & 12 & 11 \\
\hline 32 & 33 & 9 \\
\hline 13 & 19 & 6 \\
\hline 3 & 4 & 6 \\
\hline 7 & 1 & 4 \\
\hline 23 & 24 & 9 \\
\hline 25 & 31 & 8 \\
\hline 26 & 27 & 3 \\
\hline 11 & 5 & 9 \\
\hline 17 & 18 & 5 \\
\hline 12 & 6 & 10 \\
\hline 33 & 34 & 8 \\
\hline 29 & 28 & 11 \\
\hline 29 & 35 & 7 \\
\hline 30 & 36 & 3 \\
\hline
\end{tabular}




\section{Grid Topology}

Table 6.10 shows CA for links of MPSPT graph for grid topology.

Table 6.10: Channel Assignment for links of MPSPT (GT)

\begin{tabular}{|c|c|c|}
\hline \multicolumn{2}{|c|}{ Link } & Channel \\
\hline 15 & 9 & 1 \\
\hline 15 & 16 & 2 \\
\hline 15 & 14 & 3 \\
\hline 16 & 17 & 4 \\
\hline 9 & 3 & 5 \\
\hline 14 & 13 & 6 \\
\hline 17 & 18 & 7 \\
\hline 15 & 21 & 8 \\
\hline 9 & 10 & 9 \\
\hline 14 & 20 & 10 \\
\hline 16 & 22 & 11 \\
\hline 3 & 4 & 11 \\
\hline 13 & 19 & 11 \\
\hline 17 & 23 & 10 \\
\hline 18 & 24 & 11 \\
\hline 9 & 8 & 7 \\
\hline 21 & 27 & 9 \\
\hline 3 & 2 & 10 \\
\hline 10 & 11 & 6 \\
\hline 20 & 26 & 7 \\
\hline 22 & 28 & 6 \\
\hline 4 & 5 & 8 \\
\hline 19 & 25 & 9 \\
\hline 23 & 29 & 9 \\
\hline 24 & 30 & 8 \\
\hline 8 & 7 & 4 \\
\hline 27 & 33 & 5 \\
\hline 2 & 1 & 8 \\
\hline 11 & 12 & 5 \\
\hline 26 & 32 & 6 \\
\hline 28 & 34 & 7 \\
\hline 5 & 6 & 10 \\
\hline 25 & 31 & 8 \\
\hline 29 & 35 & 5 \\
\hline 30 & 36 & 7 \\
\hline
\end{tabular}




\subsection{Simulation Results in NS2 based on Throughput Analysis}

\subsubsection{Simulation Parameters}

Based on the channel assignment done by the gateway node, IEEE 802.11a channels are assigned to the links between the mesh nodes and transmission power for each radio of each mesh node is set accordingly.

The node settings in NS2 which are used during the simulation are shown in Table 6.11. The propagation model used is free space model or two-ray model depending upon the distance between the mesh nodes.

Table 6.11: Node Configuration in NS2

\begin{tabular}{|l|c|}
\hline Node Parameter & Setting \\
\hline Channel Type & Wireless Channel \\
\hline Propagation Model & Free Space / Two Ray Ground \\
\hline Network Interface Type & S02.11 \\
\hline Mac Type & Priority Queue \\
\hline Interface Queue Type & LL \\
\hline Link Layer Type & 50 \\
\hline Interface Queue Length (packets) & \\
\hline
\end{tabular}

The physical layer settings of the node which are used during the simulation are shown in Table 6.12. Note that out of the 12 available non-overlapping 802.11a channels, 11 channels are used for data traffic and channel 12 is used for control.

As mentioned earlier, the maximum transmission power for all the radios is 27 $\mathrm{dBm}$. In the CCA and SRSC schemes, MRs do not control their power, transmit with the same maximum power $(27 \mathrm{dBm})$, and use AODV (Ad hoc On-Demand Distance Vector) routing protocol as their routing agent.

The value of the carrier sense threshold is set such that the carrier sensing range (interference range) is twice the packet reception range (transmission range). In NS2, packet transmissions in the carrier sensing range of a receiver are considered as potential sources of interference that can lead to packet collisions. 
The packet capture threshold represents the Signal-to-Interference Ratio (SIR) such that if the power of the incoming packet is smaller than the power of the packet currently being received by at least the capture threshold, then the new packet is ignored.

Table 6.12: Physical Layer Node Configuration in NS2

\begin{tabular}{|c|c|}
\hline Physical Layer Parameter & Setting \\
\hline Antenna Type & Omni Antenna \\
\hline TX Antenna Height (meters) & 3 \\
\hline RX Antenna Height (meters) & 3 \\
\hline Gain of TX Antenna (dB) & 1 \\
\hline Gain of RX Antenna (dB) & 1 \\
\hline Packet Capture Threshold (SIR) (dB) & 10 \\
\hline Packet Reception Threshold (watts) & $3.16227 \mathrm{e}-10$ \\
\hline Carrier Sense Threshold (watts) & $7.90569 \mathrm{e}-11$ \\
\hline System Loss Factor & 1 \\
\hline Transmit Power (W) & $\begin{array}{l}\text { calculated for each radio interface of each mesh } \\
\text { node depending upon the channel assigned by the } \\
\text { gateway and the distance to the neighbor }\end{array}$ \\
\hline Channel Settings & $\begin{array}{c}\text { 5 GHz channels } \\
\text { Channel } 1-5.18 \mathrm{GHz} \\
\text { Channel } 2-5.20 \mathrm{GHz} \\
\text { Channel } 3-5.22 \mathrm{GHz} \\
\text { Channel } 4-5.24 \mathrm{GHz} \\
\text { Channel } 5-5.26 \mathrm{GHz} \\
\text { Channel } 6-5.28 \mathrm{GHz} \\
\text { Channel } 7-5.30 \mathrm{GHz} \\
\text { Channel } 8-5.32 \mathrm{GHz} \\
\text { Channel } 9-5.745 \mathrm{GHz} \\
\text { Channel } 10-5.765 \mathrm{GHz} \\
\text { Channel } 11-5.785 \mathrm{GHz} \\
\text { according to the channel assigned by the gateway }\end{array}$ \\
\hline
\end{tabular}


The 802.11a MAC layer settings of the node which are used during the simulation are shown in Table 6.13.

Table 6.13: MAC Layer Node Configuration in NS2

\begin{tabular}{|l|c|}
\hline MAC Layer Parameter & Setting \\
\hline Minimum Contention Window & 15 \\
\hline Maximum Contention Window & 923 \\
\hline Slot Time (micro seconds) & 16 \\
\hline SIFS period (micro seconds) & 96 \\
\hline Preamble Length (bits) & 24 \\
\hline PLCP Header Length (bits) & 6 \\
\hline PLCP Data Rate (Mbps) & 6 \\
\hline Basic Rate (Mbps) & 54 \\
\hline Data Rate (Mbps) & 10192 (disabled) \\
\hline RTS/CTS Threshold (bytes) & \\
\hline
\end{tabular}

All the mesh nodes at the periphery of the network send traffic to the gateway. Each of these nodes generates an $8 \mathrm{Mbps}$ Constant Bit Rate (CBR) traffic stream consisting of 1024 byte packets and sends data to the gateway node at the same time. They do not stop transmitting until the end of the simulation which is 600 seconds (10 minutes). The related traffic generation parameters used during the simulation are shown in Table 6.14.

Table 6.14: Traffic Generation Parameters in NS2

\begin{tabular}{|l|c|}
\hline Traffic Generation Parameter & Setting \\
\hline Traffic Type & User Datagram Protocol (UDP) \\
\hline Transport Agent & 1 \\
\hline Start Time (seconds) & 600 \\
\hline Stop Time (seconds) & 1024 \\
\hline Packet Size (bytes) & 0.001 \\
\hline Packet Inter-arrival time (seconds) & \\
\hline
\end{tabular}




\subsubsection{Simulation Results for a 36-node Network}

The Average Throughput (AT) in Mega bits per second at the gateway node is calculated using the following formula:

$$
\boldsymbol{A T}=\frac{T P R \times 8 \times 1024}{(\text { TrafficStopTime }- \text { TrafficStartTime }) \times 1 \times 10^{6}}
$$

Note that TPR is the Total Packets Received in (8).

\section{A. Random Topology}

\section{i) Ten Different Random Topologies}

Table 6.15 shows the average throughput of all schemes for ten random topologies for a network of 36 nodes. Note that AT is the Average Throughput in Mbps and $\mathrm{x}$ indicates that the resulting topology is not connected.

Table 6.15: AT (Mbps) for ten random topologies - 36-node network

\begin{tabular}{|c|c|c|c|c|}
\hline AT Scheme & TICA & & CCA & SRSC \\
\hline \multirow{2}{*}{ AT SEED 1} & Select 2 for less than 2 TCA & $x$ & \multirow{2}{*}{27.96} & \multirow{2}{*}{15.44} \\
\hline & Select 3 for less than 3 TCA & 49.33 & & \\
\hline \multirow{2}{*}{ AT SEED 2} & Select 2 for less than 2 TCA & $x$ & \multirow{2}{*}{22.95} & \multirow{2}{*}{13.06} \\
\hline & Select 3 for less than 3 TCA & 34.97 & & \\
\hline \multirow{2}{*}{ AT SEED 3} & Select 2 for less than 2 TCA & $x$ & \multirow{2}{*}{6.33} & \multirow{2}{*}{0.86} \\
\hline & Select 3 for less than 3 TCA & 75.12 & & \\
\hline \multirow{2}{*}{ AT SEED 4} & Select 2 for less than 2 TCA & 25.52 & \multirow{2}{*}{17.36} & \multirow{2}{*}{2.60} \\
\hline & Select 3 for less than 3 TCA & 53.31 & & \\
\hline \multirow{2}{*}{ AT SEED 5} & Select 2 for less than 2 TCA & $x$ & \multirow{2}{*}{14.79} & \multirow{2}{*}{3.20} \\
\hline & Select 3 for less than 3 TCA & 43.82 & & \\
\hline \multirow{2}{*}{ AT SEED 6} & Select 2 for less than 2 TCA & $x$ & \multirow{2}{*}{19.36} & \multirow{2}{*}{3.72} \\
\hline & Select 3 for less than 3 TCA & 41.13 & & \\
\hline \multirow{2}{*}{ AT SEED 7} & Select 2 for less than 2 TCA & 49.48 & \multirow{2}{*}{5.42} & \multirow{2}{*}{0.62} \\
\hline & Select 3 for less than 3 TCA & 49.50 & & \\
\hline \multirow{2}{*}{ AT SEED 8} & Select 2 for less than 2 TCA & $x$ & \multirow{2}{*}{20.51} & \multirow{2}{*}{8.98} \\
\hline & Select 3 for less than 3 TCA & 39.29 & & \\
\hline \multirow{2}{*}{ AT SEED 9} & Select 2 for less than 2 TCA & $x$ & \multirow{2}{*}{19.28} & \multirow{2}{*}{6.37} \\
\hline & Select 3 for less than 3 TCA & 57.32 & & \\
\hline \multirow{2}{*}{ AT SEED 10} & Select 2 for less than 2 TCA & $x$ & \multirow{2}{*}{5.64} & \multirow{2}{*}{1.24} \\
\hline & Select 3 for less than 3 TCA & 38.52 & & \\
\hline
\end{tabular}


Figure 6.7 shows a graphical comparison of the average throughput of all schemes for ten random topologies.

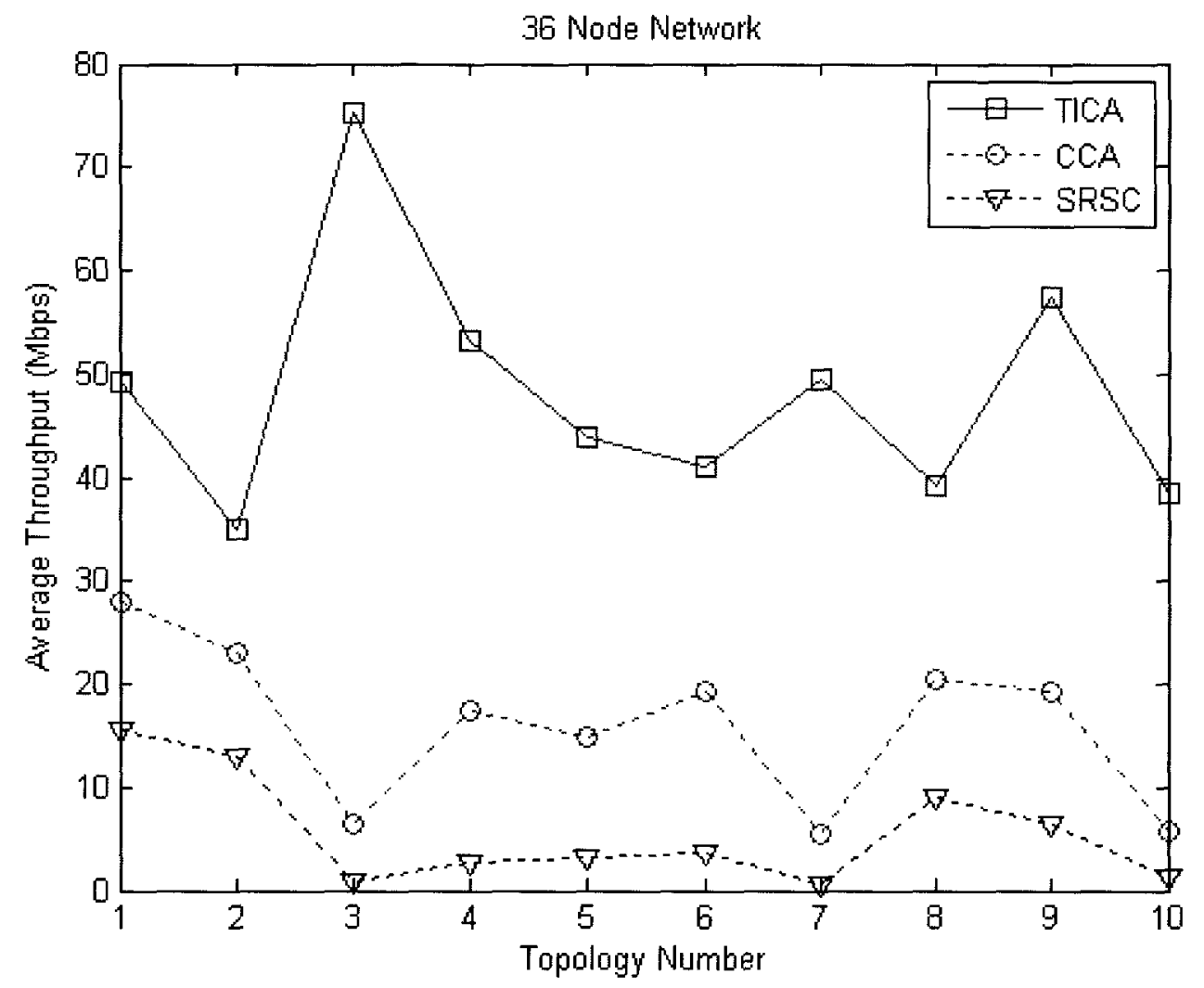

Figure 6.7: AT for Ten Random Topologies - 36-node network

The results in Figure 6.7 clearly indicate that the proposed algorithm TICA significantly outperforms other schemes for all different random topologies.

\section{ii) Throughput Analysis for Different Random Topologies}

For a topology, the maximum traffic that can reach the gateway depends on the number of links emanating from the gateway and the number of traffic sources using those links. The maximum traffic that can reach the gateway over a link emanating from the gateway is $8.192 \mathrm{Mbps}$ if one traffic source is using that link, 16.384 Mbps in case of two sources, 24.576 Mbps in case of three sources and 24.8 Mbps in case of four or more sources.

The MPSPTs, for the different random and controlled random topologies for a 36node network, showing the number of links emanating from the gateway and the number of sources using these links, are shown in Appendices C.1 and C.2. The maximum traffic 
that can reach the gateway limits the actual throughput achieved by TICA. Table 6.16 shows the maximum traffic that can reach the gateway and the actual throughput achieved by TICA for different random topologies.

Table 6.16: Throughput Analysis for different RTs - 36-node network

\begin{tabular}{|c|c|c|c|c|c|}
\hline $\begin{array}{c}\text { RT } \\
\text { Seed }\end{array}$ & $\begin{array}{c}\text { GW } \\
\text { links }\end{array}$ & $\begin{array}{c}\text { Traffic sources } \\
\text { using each } \\
\text { GW link }\end{array}$ & $\begin{array}{c}\text { Max. traffic on each GW } \\
\text { link } \\
\text { (Mbps) }\end{array}$ & $\begin{array}{c}\text { Max. traffic } \\
\text { at GW } \\
\text { (Mbps) }\end{array}$ & $\begin{array}{c}\text { Actual Throughput } \\
\text { at GW with TICA } \\
\text { (Mbps) }\end{array}$ \\
\hline 1 & 4 & $1,1,1,10$ & $8.192,8.192,8.192,24.8$ & 49.376 & 49.33 \\
\hline 2 & 2 & 3,11 & $24.576,24.8$ & 49.376 & 34.97 \\
\hline 3 & 4 & $1,3,5,5$ & $8.192,24.576,24.8,24.8$ & 82.368 & 75.12 \\
\hline 4 & 3 & $2,4,5$ & $16.384,24.8,24.8$ & 65.984 & 53.31 \\
\hline 5 & 2 & 3,8 & $24.576,24.8$ & 49.376 & 43.82 \\
\hline 6 & 3 & $1,1,9$ & $8.192,8.192,24.8$ & 41.184 & 41.13 \\
\hline 7 & 2 & 7,4 & $24.8,24.8$ & 49.60 & 49.50 \\
\hline 8 & 4 & $1,3,1,7$ & $8.192,24.576,8.192,24.8$ & 65.76 & 39.29 \\
\hline 9 & 3 & $1,4,7$ & $8.192,24.8,24.8$ & 57.792 & 57.32 \\
\hline 10 & 2 & 4,10 & $24.8,24.8$ & 49.60 & 38.52 \\
\hline
\end{tabular}

The actual throughput, achieved by CCA and SRSC schemes, depends on the location of the gateway and the number of traffic sources located close to the gateway as compared to other traffic sources. Therefore, in seeds of the random topology where the gateway is located such that some traffic sources are located close to the gateway as compared to other traffic sources as shown in Appendix C.1, the CCA and SRSC schemes result in a relatively high throughput. In such cases, since some traffic sources are located close to the gateway and outside the interference range of other traffic sources, so their traffic experiences less interference which leads to a relatively high throughput at the gateway.

The placement of the nodes and the length of links in the MPSPT of a topology affects the interference range and hence the channel assignment. In random and controlled random topologies, the random placement of nodes results in variation in the length of links in the MPSPT. This results in LICs which may cause significant interference in the network and degrade the overall throughput. 
In case of second, eight and tenth seeds of the random topology, the resulting LICs cause significant interference and result in a relatively lower throughput with TICA as shown in Table 6.16.

\section{iii) Throughput Improvement Factor}

The average Throughput Improvement Factor (TIF), over ten different random topologies, showing the throughput improvement with TICA as compared to CCA and SRSC schemes is shown in Table 6.17.

Table 6.17: TIF (RT) - 36-node network

\begin{tabular}{|c|c|c|}
\hline Scheme & Average Throughput (Mbps) & TIF \\
\hline TICA & 48.23 & - \\
\hline CCA & 15.96 & 3.021 \\
\hline SRSC & 5.61 & 8.597 \\
\hline
\end{tabular}

\section{B. Controlled Random Topology}

\section{i) Ten Different Controlled Random Topologies}

Table 6.18 shows the average throughput of all schemes for ten controlled random topologies for a network of 36 nodes. Note that AT is the Average Throughput in Mbps and $\mathrm{x}$ indicates that the resulting topology is not connected.

The "Select 1 for less than 1" TCA leads to a connected network in case of gird topology which is due to the symmetric nature of the grid topology. The "Select 1 for less than 1" TCA leads to a disconnected network in case of random and controlled random topologies which is due to the random nature of these topologies. The "Select 2 for less than 2" TCA leads to a disconnected network in case of random and controlled random topologies in most cases. However, in cases where it leads to a connected network for these topologies, the results show that the average throughput with "Select 2 for less than 2" TCA is less than that with "Select 3 for less than 3" TCA for TICA. This is due to the fact that the "Select 2 for less than 2" TCA results in a connectivity graph with low average node degree in TICA which leads to low throughput. Therefore, if the "Select 1 for less than 1" TCA leads to a disconnected network, this means that the topology is either random or controlled random. In this case, I have concluded that the next higher TCA for the gateway should be "Select 3 for less than 3. " 
Table 6.18: AT (Mbps) for ten controlled random topologies - 36-node network

\begin{tabular}{|c|c|c|c|c|}
\hline AT Scheme & TICA & & CCA & SRSC \\
\hline \multirow{2}{*}{ AT SEED1 } & Select 2 for less than 2 TCA & $x$ & \multirow{2}{*}{5.76} & \multirow{2}{*}{0.66} \\
\hline & Select 3 for less than 3 TCA & 71.70 & & \\
\hline \multirow{2}{*}{ AT SEED 2} & Select 2 for less than 2 TCA & 32.78 & \multirow{2}{*}{5.82} & \multirow{2}{*}{0.64} \\
\hline & Select 3 for less than 3 TCA & 47.43 & & \\
\hline \multirow{2}{*}{ AT SEED 3} & Select 2 for less than $2 \mathrm{TCA}$ & 54.69 & \multirow{2}{*}{4.04} & \multirow{2}{*}{0.47} \\
\hline & Select 3 for less than 3 TCA & 63.54 & & \\
\hline \multirow{2}{*}{ AT SEED 4} & Select 2 for less than 2 TCA & $\mathrm{x}$ & \multirow{2}{*}{3.71} & \multirow{2}{*}{0.33} \\
\hline & Select 3 for less than 3 TCA & 77.43 & & \\
\hline \multirow{2}{*}{ AT SEED 5} & Select 2 for less than 2 TCA & 57.20 & \multirow{2}{*}{5.66} & \multirow{2}{*}{1.00} \\
\hline & Select 3 for less than 3 TCA & 69.81 & & \\
\hline \multirow{2}{*}{ AT SEED 6} & Select 2 for less than 2 TCA & $\bar{x}$ & \multirow{2}{*}{6.42} & \multirow{2}{*}{0.57} \\
\hline & Select 3 for less than 3 TCA & 75.49 & & \\
\hline \multirow{2}{*}{ AT SEED 7} & Select 2 for less than 2 TCA & $x$ & \multirow{2}{*}{4.54} & \multirow{2}{*}{0.62} \\
\hline & Select 3 for less than 3 TCA & 62.90 & & \\
\hline \multirow{2}{*}{ AT SEED 8} & Select 2 for less than $2 \mathrm{TCA}$ & $x$ & \multirow{2}{*}{6.91} & \multirow{2}{*}{0.54} \\
\hline & Select 3 for less than 3 TCA & 49.50 & & \\
\hline \multirow{2}{*}{ AT SEED 9} & Select 2 for less than 2 TCA & $x$ & \multirow{2}{*}{6.53} & \multirow{2}{*}{0.84} \\
\hline & Select 3 for less than 3 TCA & 51.33 & & \\
\hline \multirow{2}{*}{ AT SEED 10} & Select 2 for less than 2 TCA & $x$ & \multirow{2}{*}{4.61} & \multirow{2}{*}{0.77} \\
\hline & Select 3 for less than 3 TCA & 44.67 & & \\
\hline
\end{tabular}

Figure 6.8 shows a graphical comparison of average throughput of all schemes for ten controlled random topologies. The results in Figure 6.8 clearly indicate that the proposed algorithm TICA significantly outperforms other schemes for all different controlled random topologies.

\section{ii) Throughput Analysis for Different Controlled Random Topologies}

The maximum traffic that can reach the gateway and the actual throughput achieved by TICA for different controlled random topologies is shown in Table 6.19. 


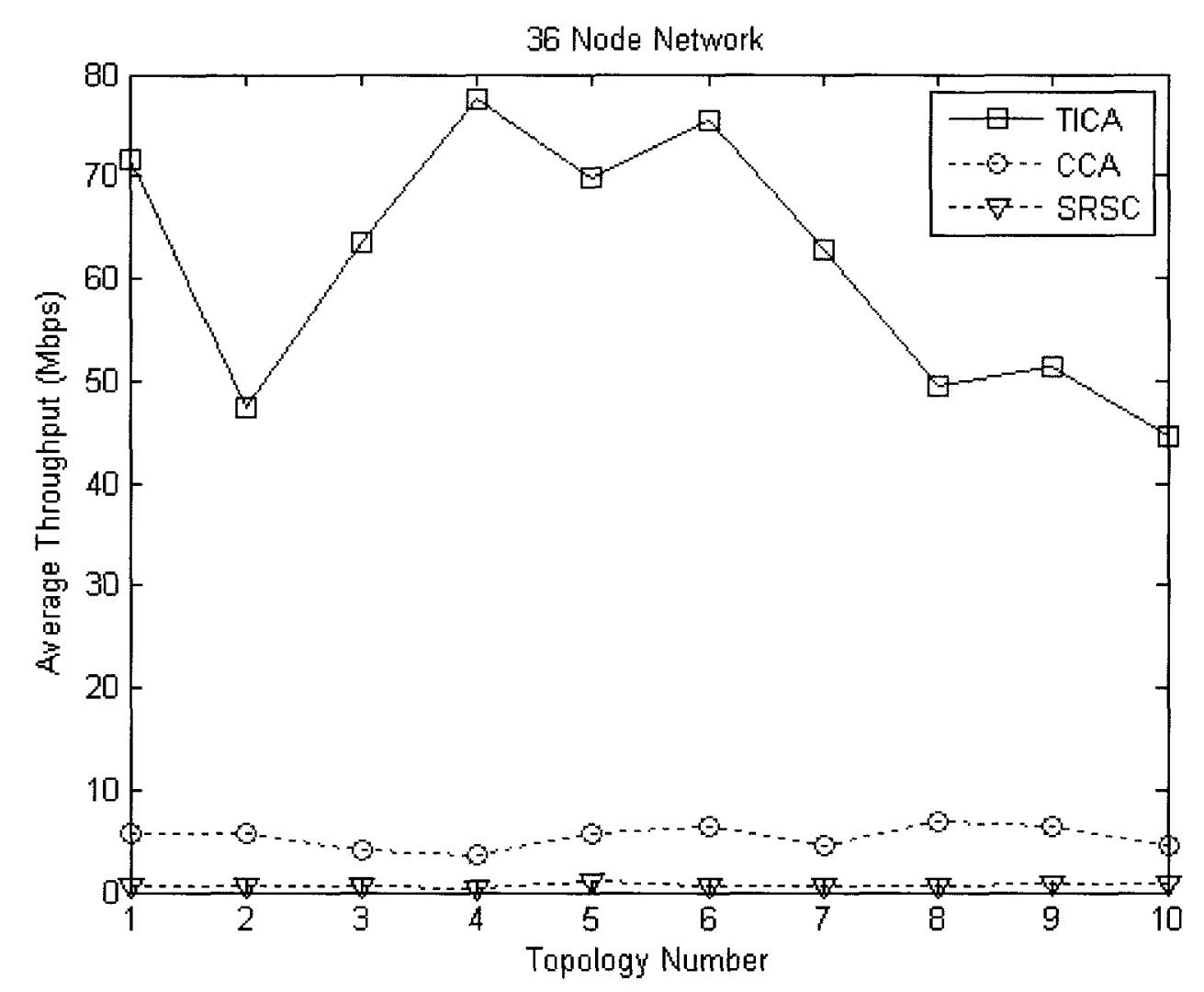

Figure 6.8: AT for Ten Controlled Random Topologies - 36-node network

Table 6.19: Throughput Analysis for different CRTs - 36-node network

\begin{tabular}{|c|c|c|c|c|c|}
\hline $\begin{array}{c}\text { CRT } \\
\text { Seed }\end{array}$ & $\begin{array}{c}\text { GW } \\
\text { links }\end{array}$ & $\begin{array}{c}\text { Traffic } \\
\text { sources } \\
\text { using each } \\
\text { GW link }\end{array}$ & $\begin{array}{c}\text { Max. traffic on each GW link } \\
\text { (Mbps) }\end{array}$ & $\begin{array}{c}\text { Max. traffic at } \\
\text { GW } \\
\text { (Mbps) }\end{array}$ & $\begin{array}{c}\text { Actual Throughput } \\
\text { at GW with TICA } \\
\text { (Mbps) }\end{array}$ \\
\hline 1 & 3 & $4,5,4$ & $24.8,24.8,24.8$ & 74.40 & 71.70 \\
\hline 2 & 3 & $4,4,7$ & $24.8,24.8,24.8$ & 74.40 & 47.43 \\
\hline 3 & 3 & $4,3,7$ & $24.8,24.576,24.8$ & 82.592 & 63.54 \\
\hline 4 & 4 & $4,1,4,6$ & $24.8,8.192,24.8,24.8$ & 74.176 & 77.43 \\
\hline 5 & 4 & $1,2,6,4$ & $8.192,16.384,24.8,24.8$ & 90.56 & 69.81 \\
\hline 6 & 4 & $4,6,2,3$ & $24.8,24.8,16.384,24.576$ & 65.984 & 62.90 \\
\hline 7 & 3 & $2,6,5$ & $16.384,24.8,24.8$ & 49.60 & 49.50 \\
\hline 8 & 2 & 5,7 & $24.8,24.8$ & 73.952 & 51.33 \\
\hline 9 & 3 & $3,3,6$ & $24.576,24.576,24.8$ & 49.60 & 44.67 \\
\hline 10 & 2 & 4,9 & $24.8,24.8$ & & 79.49 \\
\hline
\end{tabular}


In all seeds of the controlled random topology as shown in Appendix C.2, the gateway is located such that no traffic sources are located close to the gateway and outside the interference range of other traffic sources. Therefore, in CCA and SRSC schemes, traffic from all traffic sources experiences interference which leads to a relatively low throughput for these schemes.

In the second and ninth seeds of the controlled random topology, the resulting LICs cause significant interference in the network and result in a relatively low throughput with TICA as shown in Table 6.19.

\section{iii) Throughput Improvement Factor}

The average TIF, over ten different controlled random topologies, showing the throughput improvement with TICA as compared to CCA and SRSC schemes is shown in Table 6.20.

Table 6.20: TIF (CRT) - 36-node network

\begin{tabular}{|c|c|c|}
\hline Scheme & Average Throughput (Mbps) & TIF \\
\hline TICA & 61.38 & - \\
\hline CCA & 5.40 & 11.366 \\
\hline SRSC & 0.64 & 95.906 \\
\hline
\end{tabular}

\section{Grid Topology}

\section{i) Throughput Improvement Factor}

The TIF showing the throughput improvement by TICA as compared to CCA and SRSC schemes is shown in Table 6.21.

Table 6.21: TIF (GT) - 36-node network

\begin{tabular}{|c|c|c|}
\hline Scheme & Average Throughput (Mbps) & TIF \\
\hline TICA & 70.89 & - \\
\hline CCA & 5.51 & 12.865 \\
\hline SRSC & 0.53 & 133.754 \\
\hline
\end{tabular}

\section{ii) Throughput Analysis for Grid Topology}

The maximum traffic that can reach the gateway and the actual throughput achieved by TICA for grid topology is shown in Table 6.22 . 
Table 6.22: Throughput Analysis for GT - 36-node network

\begin{tabular}{|c|c|c|c|c|c|}
\hline GT & $\begin{array}{c}\text { GW } \\
\text { links }\end{array}$ & $\begin{array}{c}\text { Traffic } \\
\text { sources using } \\
\text { each GW link }\end{array}$ & $\begin{array}{c}\text { Max. traffic on each GW } \\
\text { link } \\
\text { (Mbps) }\end{array}$ & $\begin{array}{c}\text { Max. traffic at } \\
\text { GW } \\
\text { (Mbps) }\end{array}$ & $\begin{array}{c}\text { Actual Throughput } \\
\text { at GW with TICA } \\
\text { (Mbps) }\end{array}$ \\
\hline 1 & 4 & $4,3,1,2$ & $24.8,24.576,8.192,16.384$ & 73.952 & 70.89 \\
\hline
\end{tabular}

As shown in the MPSPT for the grid topology in Figure 6.6, there are no traffic sources which are located close to the gateway and outside the interference range of other traffic sources. Therefore, in CCA and SRSC schemes, traffic from all traffic sources experiences interference which leads to a relatively low throughput for these schemes.

Since all links in the grid topology have equal length due to the symmetric nature of this topology, so the resulting LICs succeed in minimizing the interference and lead to a high throughput with TICA, as shown in Table 6.22.

\section{Throughput Comparison of All Schemes}

Figure 6.9 shows the comparison of average throughput of all schemes for the three topologies (random, controlled random and grid) for a network of 36 nodes.

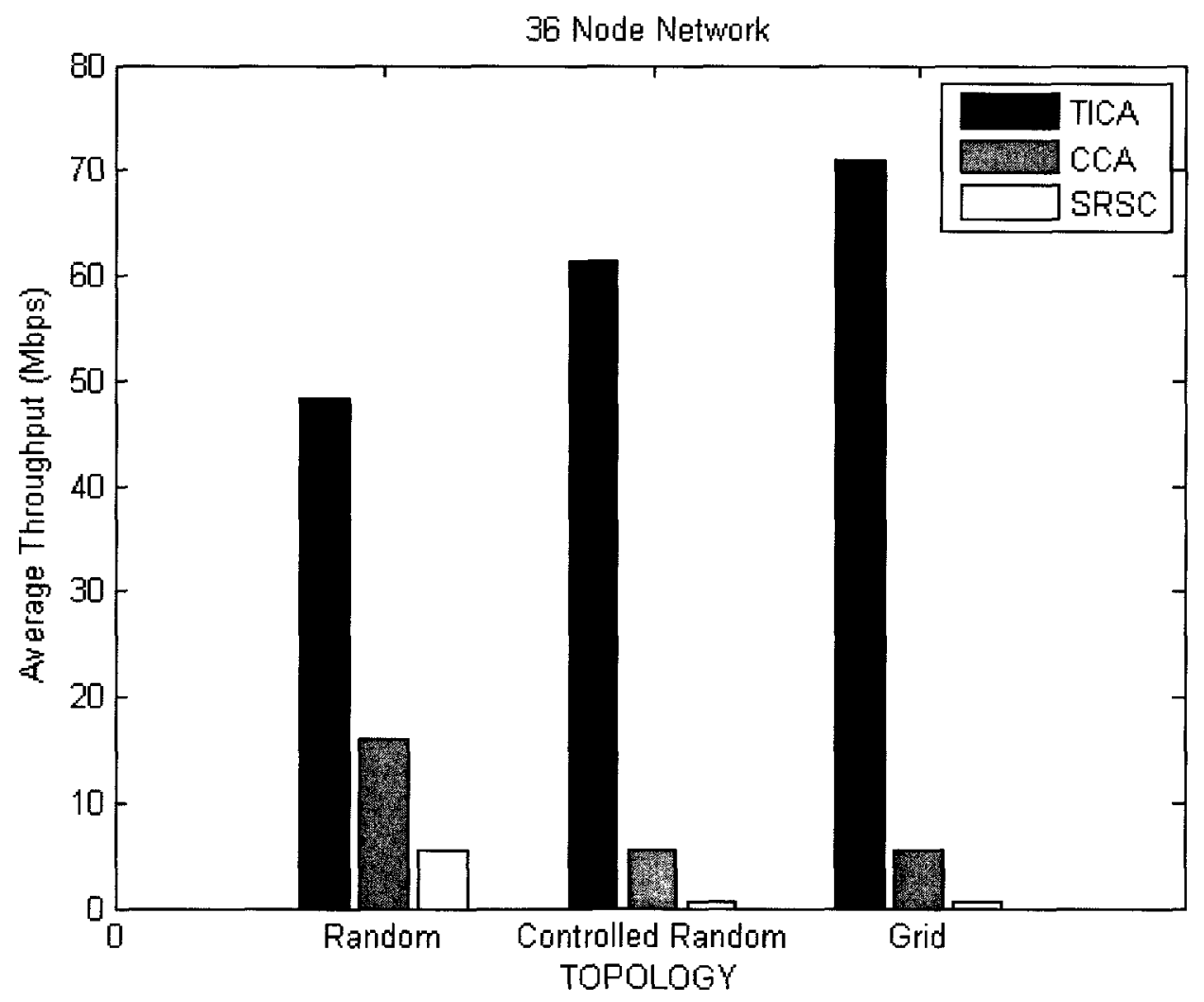

Figure 6.9: AT for all schemes - 36-node network 
Note that for random and controlled random topologies in Figure 6.9, the average throughput is the average over ten different random and controlled random topologies, respectively. Figure 6.9 shows that as compared to the CCA scheme, the throughput improvement with TICA is 3 times for random topology, 11 times for controlled random topology and 12 times for grid topology. In comparison to the SRSC scheme, the throughput improvement with TICA is 8 times for random topology, 95 times for controlled random topology and 133 times for grid topology.

The results in Figure 6.9 clearly indicate that the proposed algorithm TICA significantly outperforms other schemes for the three topologies.

\subsubsection{Simulation Results for a 144-node Network}

In this case, 144 mesh nodes are distributed in an area of 500x500 meters for the three topologies (random, controlled random and grid). Node 66 is taken as the gateway and the remaining simulation parameters are the same.

\section{A. Random Topology}

The throughput improvement by TICA as compared to CCA and SRSC schemes is shown in Table 6.23. Note that Select 4 for less than 4 TCA results in a connected network for this topology.

Table 6.23: TIF (RT) - 144-node network

\begin{tabular}{|c|c|c|}
\hline Scheme & Average Throughput (Mbps) & TIF \\
\hline TICA & 42.54 & - \\
\hline CCA & 2.43 & 17.506 \\
\hline SRSC & 0.82 & 51.878 \\
\hline
\end{tabular}

\section{B. Controlled Random Topology}

The throughput improvement by TICA as compared to CCA and SRSC schemes is shown in Table 6.24. Note that Select 3 for less than 3 TCA results in a connected network for this topology.

Table 6.24: TIF (CRT) - 144-node network

\begin{tabular}{|c|c|c|}
\hline Scheme & Average Throughput (Mbps) & TIF \\
\hline TICA & 67.79 & - \\
\hline CCA & 0.07 & 968.428 \\
\hline SRSC & 0.06 & 1129.833 \\
\hline
\end{tabular}




\section{Grid Topology}

The throughput improvement by TICA as compared to CCA and SR SC schemes is shown in Table 6.25. Note that Select 1 for less than 1 TCA results in a connected network for this topology.

Table 6.25: TIF (GT) - 144-node network

\begin{tabular}{|c|c|c|}
\hline Scheme & Average Throughput (Mbps) & TIF \\
\hline TICA & 82.42 & - \\
\hline CCA & 0.23 & 358.347 \\
\hline SRSC & 0.08 & 1030.250 \\
\hline
\end{tabular}

\section{Throughput Comparison of All Schemes}

Figure 6.10 shows a graphical comparison of average throughput of all schemes for the three different topologies (random, controlled random and grid) for a network of 144 nodes.

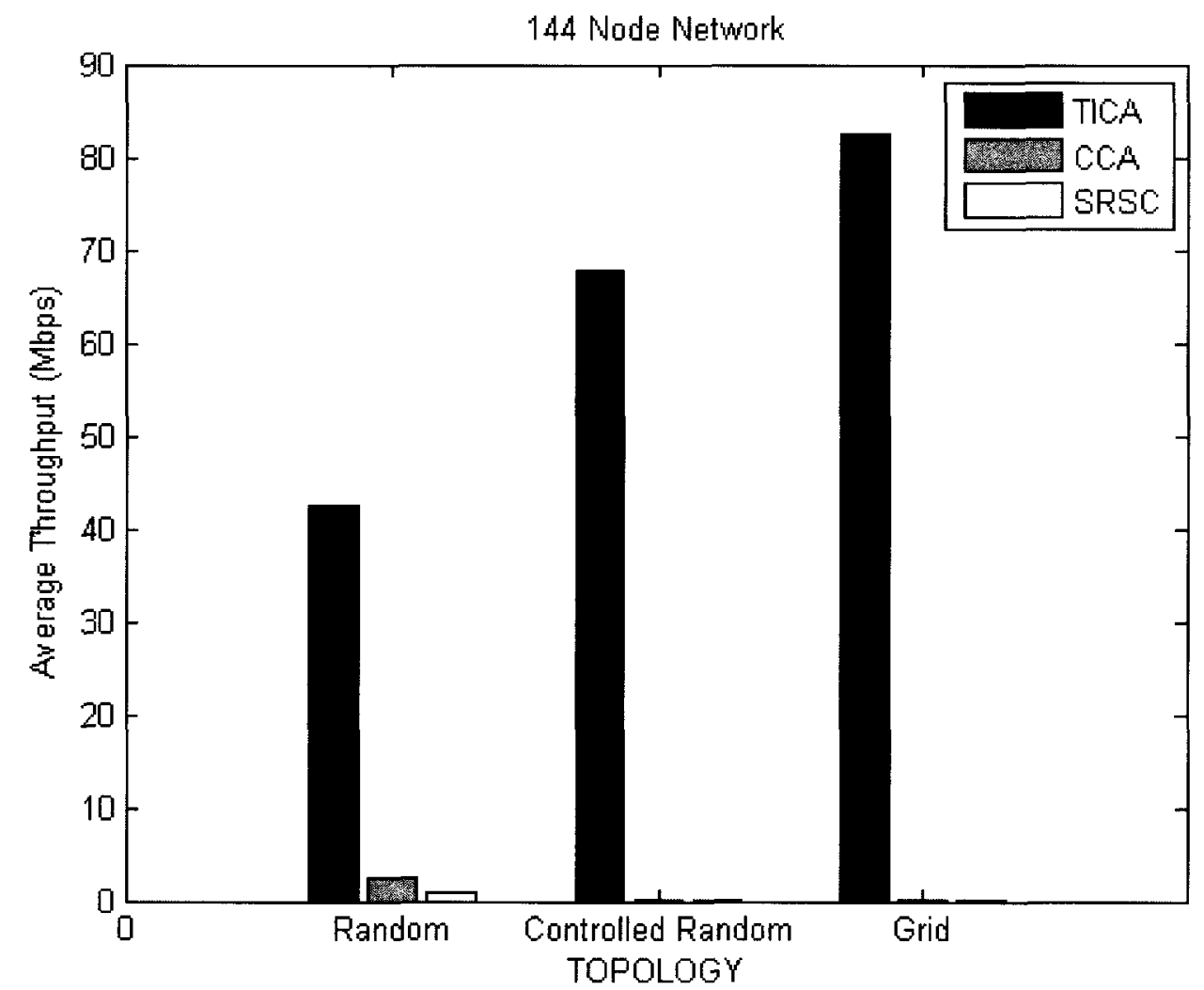

Figure 6.10: AT for all schemes - 144-node network

The results in Figure 6.10 show that TICA significantly outperforms the other schemes for the three topologies in a 144-node network. These results show that the 
throughput improvement with TICA in a large-scale network of 144 nodes, in comparison to CCA and SRSC schemes, is significantly higher than the throughput improvement with TICA in a small-scale network of 36 nodes.

The CCA and SRSC schemes perform poorly in 144-node network due to the large number of traffic sources. More traffic sources means more nodes competing for medium access. Increased contention for medium access combined with lack of power control in these schemes leads to more collisions and reduced throughput for these schemes.

\section{E. Throughput Analysis for the Three Topologies}

The maximum traffic that can reach the gateway and the actual throughput achieved by TICA for the three topologies for 144-node network is shown in Table 6.26.

Table 6.26: Throughput Analysis - 144-node network

\begin{tabular}{|c|c|c|c|c|c|}
\hline Topology & $\begin{array}{c}\text { GW } \\
\text { links }\end{array}$ & $\begin{array}{c}\text { Traffic } \\
\text { sources } \\
\text { using each } \\
\text { GW link }\end{array}$ & $\begin{array}{c}\text { Max. traffic on each GW link } \\
\text { (Mbps) }\end{array}$ & $\begin{array}{c}\text { Max. traffic } \\
\text { at GW } \\
\text { (Mbps) }\end{array}$ & $\begin{array}{c}\text { Actual Throughput } \\
\text { at GW with TICA } \\
\text { (Mbps) }\end{array}$ \\
\hline R & 3 & $1,10,31$ & $8.192,24.8,24.8$ & 57.792 & 42.54 \\
\hline CR & 3 & $6,12,26$ & $24.8,24.8,24.8$ & 74.4 & 67.79 \\
\hline$G$ & 4 & $10,5,1,6$ & $24.8,24.8,8.192,24.8$ & 82.592 & 82.42 \\
\hline
\end{tabular}

As shown in the MPSPT for the random topology for 144-node network in Appendix C.3, there are some traffic sources which are located close to the gateway and outside the interference range of other traffic sources. Therefore, in CCA and SRSC schemes, traffic from such sources experience less interference and this leads to a relatively high throughput for these schemes in random topology.

Due to high degree of randomness in the random topology, the resulting LICs cause significant interference and result in low throughput with TICA, as shown in Table 6.26 .

Since the degree of randomness in the controlled random topology is relatively low, so the resulting LICs cause relatively less interference and result in a relatively better throughput with TICA, as shown in Table 6.26.

In the grid topology, there is no variation in the length of links in MPSPT which results in LICs with minimum interference and a high throughput with TICA, as shown in Table 6.26 . 


\subsubsection{Performance Comparison of TICA with Centralized CA Schemes}

The proposed scheme, TICA, is a centralized and fixed channel assignment scheme. Therefore, it is being compared with the following two well-known centralized CA schemes.

\section{A. TICA and C-HYA}

\section{i) Simulation Scenario}

Similar to the scenario in C-HYA, the following simulation scenario is created.

- 100 mesh nodes are distributed in an area of 500x500 meters in a grid topology.

- Each mesh node can communicate with up to four neighbors.

- Twenty randomly selected mesh nodes generate CBR traffic streams consisting of UDP packets.

- The traffic sources send data to the sink (gateway) at the same time.

- The traffic sources do not stop transmitting until the end of the simulation.

- The amount of traffic generated by each source is chosen at random between 0 and 3 Mbps.

- The data rate is $54 \mathrm{Mbps}$.

- $\mathrm{RTS} / \mathrm{CTS}$ is enabled.

- The ratio between interference range (carrier sense threshold) and transmission range (packet reception threshold) is fixed at two.

\section{ii) Simulation Assumptions}

The following simulation parameters are assumed.

- The inter router distance is 50 meters.

- The maximum transmission range for all mesh nodes is set to 50 meters and the maximum transmission power is set accordingly.

- The minimum receiver sensitivity (RxThresh) is set to $-65 \mathrm{dBm}\left(3.16227 \times 10^{-10}\right.$ Watts) in order to achieve a maximum data rate of $54 \mathrm{Mbps}$ supported by IEEE 802.11a at a maximum transmission range of 50 meters.

- Node 45 is selected as the gateway due to its central location, as shown in the MPSPT in Appendix C.4. 
- The mesh nodes use AODV routing protocol as their routing agent in CCA and SRSC schemes.

- The simulation duration is 600 seconds (10 minutes).

- The packet size is set to 1024 bytes.

iii) Simulation Parameters

The simulation parameters for this scenario are shown in Table 6.27.

Table 6.27: Simulation parameters

\begin{tabular}{|l|c|}
\hline Parameter & Setting \\
\hline Packet Reception Threshold (watts) & $3.16227 \mathrm{e}-10$ \\
\hline Carrier Sense Threshold (watts) & $7.90569 \mathrm{e}-11$ \\
\hline Maximum Transmit Power (watts) & 0.0457 \\
\hline Maximum Transmission Range (meters) & 50 \\
\hline Basic Rate (Mbps) & 54 \\
\hline Data Rate (Mbps) & 512 (enabled) \\
\hline RTS/CTS Threshold (bytes) & Constant Bit Rate (CBR) \\
\hline Traffic Type & User Datagram Protocol (UDP) \\
\hline Transport Agent & 1024 \\
\hline Start Time (seconds) & Random \\
\hline Stop Time (seconds) & \\
\hline Packet Size (bytes) & \\
\hline Packet Inter-arrival time (seconds) & \\
\hline
\end{tabular}

\section{iv) Simulation Results}

The throughput improvement by TICA as compared to CCA and SRSC schemes for ten different Traffic Profile (TP) seeds is shown in Table 6.28. In each TP seed, twenty mesh nodes are selected randomly as traffic sources and the amount of traffic generated by each source is chosen at random between 0 and $3 \mathrm{Mbps}$. 
Table 6.28: AT (Mbps) with ten different TPs

\begin{tabular}{|c|c|c|c|}
\hline ST Scheme & TICA & CCA & SRSC \\
\hline AT for TP seed 1 & 31.33 & 14.39 & 3.52 \\
\hline AT for TP seed 2 & 28.64 & 16.22 & 4.95 \\
\hline AT for TP seed 3 & 32.23 & 14.32 & 3.77 \\
\hline AT for TP seed 4 & 27.37 & 14.89 & 4.59 \\
\hline AT for TP seed 5 & 31.36 & 14.72 & 3.83 \\
\hline AT for TP seed 6 & 30.59 & 16.34 & 4.00 \\
\hline AT for TP seed 7 & 24.34 & 14.84 & 3.47 \\
\hline AT for TP seed 8 & 27.34 & 15.29 & 3.62 \\
\hline AT for TP seed 9 & 34.11 & 14.21 & 3.63 \\
\hline AT for TP seed 10 & 28.69 & 15.58 & 4.48 \\
\hline
\end{tabular}

\section{v) Comparison of Results}

The normalized throughput of TICA and SRSC schemes is shown in Figure 6.11. The normalized throughput is obtained after normalizing the results with the throughput obtained from the SRSC scheme.

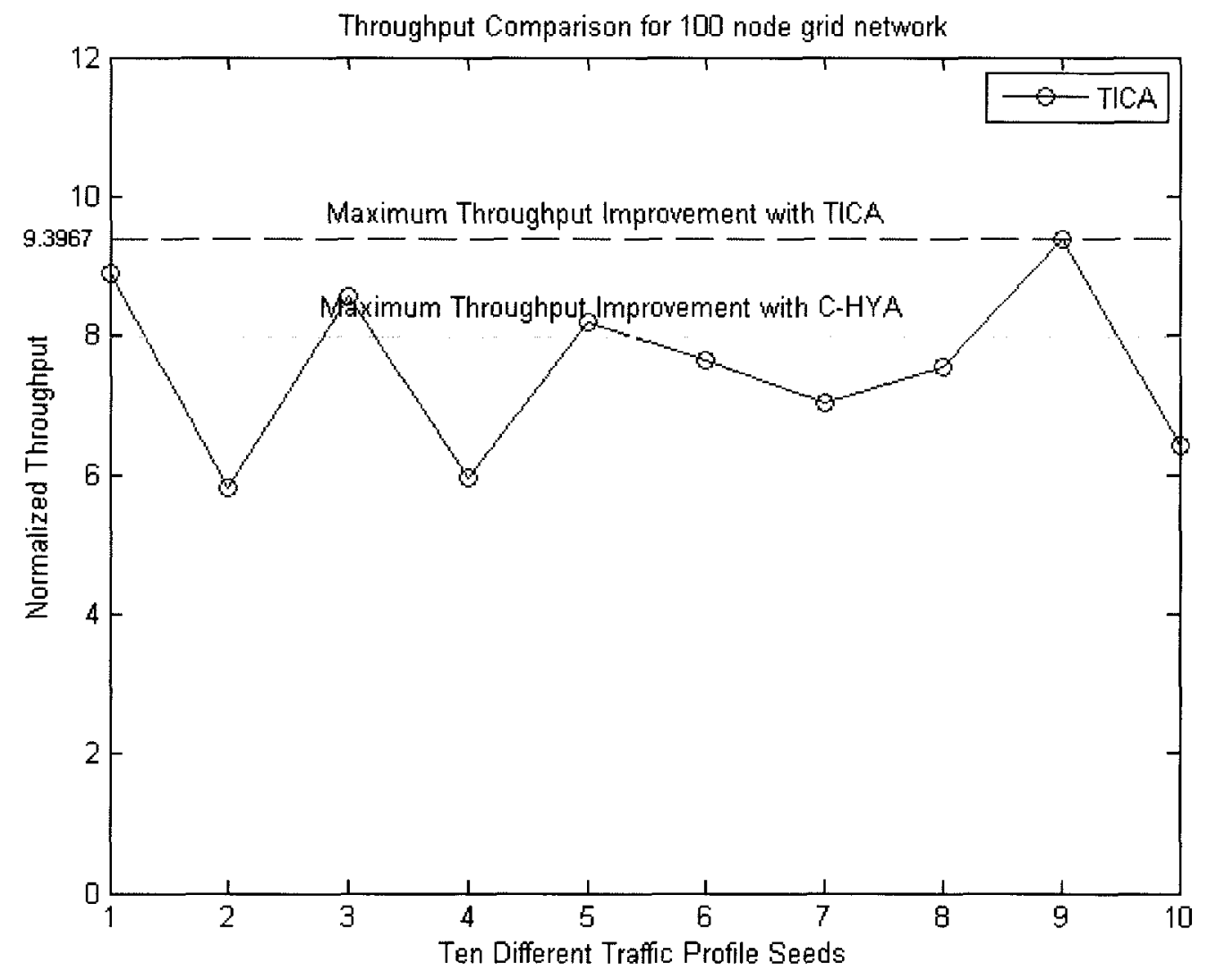

Figure 6.11: Normalized Throughput with ten different TPs 
For a 10x10 grid network of 100 nodes where UDP-based CBR traffic flows from 20 pairs of randomly chosen ingress-egress nodes interfere with each other, the throughput improvement with C-HYA is as high as 8 times in comparison with the SRSC scheme [18]. The throughput improvement with TICA for a similar scenario, where twenty randomly selected mesh nodes send UDP-based CBR traffic to the gateway at the same time, is as high as 9 times in comparison with the SRSC scheme, as shown in Figure 6.11. Hence, TICA has shown a better performance as compared to C-HYA for the same scenario where the transmission range of mesh nodes in CCA and SRSC schemes is controlled by controlling their transmission power.

\section{B. TICA and BFS-CA}

\section{i) Simulation Scenario}

Similar to the scenario in BFS-CA, the following simulation scenario is created.

- 30 mesh nodes are distributed in an area of 500x500 meters in a grid topology.

- The inter router distance is 75 meters.

- The maximum transmission power of all mesh nodes is $18 \mathrm{dBm}$.

- Ten randomly selected mesh nodes at the periphery of the network send FTP traffic to the gateway.

- Each of these sources generates an FTP traffic stream consisting of TCP packets and send data to the gateway at the same time.

- The source nodes do not stop transmitting until the end of the simulation.

- Since in BFS-CA, the mesh router which is approximately in the center of the topology is selected as the gateway, so mesh Node 13 is selected as the gateway due to its central location, as shown in the MPSPT in Appendix C.5.

- RTS/CTS is disabled.

\section{ii) Simulation Assumptions}

The following simulation parameters are assumed.

- The maximum transmission range for all mesh nodes is 75 meters.

- The maximum data rate is set to $36 \mathrm{Mbps}$ based on the maximum transmission power and the inter router distance. 
- The minimum receiver sensitivity (RxThresh) is set to $-67.2 \mathrm{dBm}\left(1.90546 \times 10^{-10}\right.$ Watts) in order to achieve a maximum data rate of $36 \mathrm{Mbps}$ supported by IEEE $802.11 \mathrm{a}$ at a maximum transmission range of 75 meters.

- The mesh nodes use AODV routing protocol as their routing agent in CCA and SRSC schemes.

- The packet size is set to 1024 bytes.

- The interference range is set as twice the transmission range.

- The simulation duration is 600 seconds (10 minutes).

iii) Simulation Parameters

The simulation parameters for this scenario are given in Table 6.29.

Table 6.29: Simulation parameters

\begin{tabular}{|l|c|}
\hline Parameter & Setting \\
\hline Packet Reception Threshold (watts) & $1.90546 \mathrm{e}-10$ \\
\hline Carrier Sense Threshold (watts) & $4.76365 \mathrm{e}-11$ \\
\hline Maximum Transmit Power (watts) & 0.0631 \\
\hline Maximum Transmission Range (meters) & 75 \\
\hline Basic Rate (Mbps) & \\
\hline Data Rate (Mbps) & 10192 (disabled) \\
\hline RTS/CTS Threshold (bytes) & File Transfer Protocol (FTP) \\
\hline Traffic Type & Transmission Control Protocol (TCP) \\
\hline Transport Agent & 1 \\
\hline Start Time (seconds) & \\
\hline Stop Time (seconds) & \\
\hline Packet Size (bytes) & 1024 \\
\hline
\end{tabular}

\section{iv) Simulation Results}

The throughput improvement by TICA as compared to CCA and SRSC schemes for ten different TP seeds is shown in Table 6.30. In each TP seed, ten mesh nodes at the periphery of the network are selected randomly as FTP traffic sources. 
Table 6.30: AT (Mbps) with ten different TPs

\begin{tabular}{|l|l|l|l|}
\hline AT Scheme & TICA & CCA & SRSC \\
\hline AT for TP seed 1 & 12.80 & 7.79 & 2.29 \\
\hline AT for TP seed 2 & 12.80 & 7.07 & 2.37 \\
\hline AT for TP seed 3 & 12.60 & 5.78 & $\mathbf{1 . 8 2}$ \\
\hline AT for TP seed 4 & 12.75 & 6.61 & 2.25 \\
\hline AT for TP seed 5 & 12.96 & 7.41 & 4.56 \\
\hline AT for TP seed 6 & 12.96 & 8.08 & 4.58 \\
\hline AT for TP seed 7 & 12.64 & 8.05 & 1.88 \\
\hline AT for TP seed 8 & $\mathbf{1 2 . 7 6}$ & 7.37 & 2.23 \\
\hline AT for TP seed 9 & 12.91 & 9.09 & 4.01 \\
\hline AT for TP seed 10 & $\mathbf{1 2 . 7 6}$ & 5.93 & 2.19 \\
\hline
\end{tabular}

\section{v) Comparison of Results}

The normalized throughput of TICA and CCA schemes is shown in Figure 6.12. The normalized throughput is obtained after normalizing the results with the throughput obtained from the CCA scheme.

Throughput Comparison for 30 node grid network

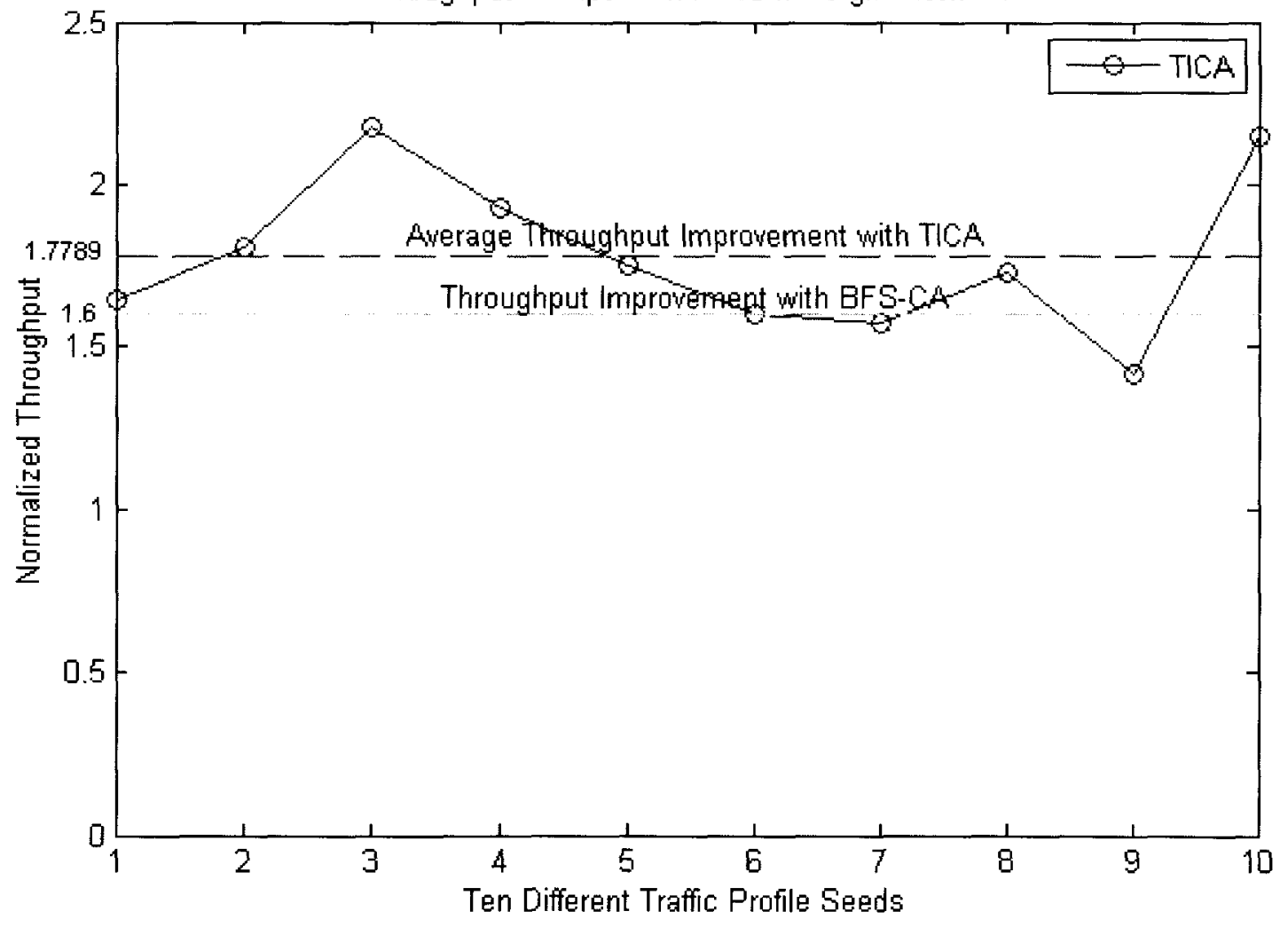

Figure 6.12: Normalized Throughput with ten different TPs 
For a grid network of 30 mesh nodes where 10 randomly selected traffic sources at the periphery send TCP-based FTP traffic to the gateway at the same time, the throughput improvement with BFS-CA is 60\% in comparison with the CCA scheme [27]. The average throughput improvement with TICA for a similar scenario for ten different TP seeds is $77 \%$ in comparison with the CCA scheme, as shown in Figure 6.12. Hence, TICA has shown a better performance as compared to BFS-CA for the same scenario where the transmission range of mesh nodes in CCA and SRSC schemes is controlled by controlling their receiver sensitivity.

\subsection{Features' Comparison of Related CA Schemes}

The important features of the related CA schemes are summarized in Table 6.31. They include channel switching, topology control, power control, knowledge of traffic load, connectivity, fault tolerance and CA control.

The CA schemes, such as C-HYA [18], MesTiC [21] and D-HYA [24], require the traffic load to be known before assigning channels, whereas TICA requires no such knowledge for channel assignment.

HMCP scheme [25] [26] requires radios to switch between channels on a perpacket basis. In such cases, time synchronization and coordination between mesh nodes is required which is not needed in TICA.

BFS-CA scheme [27] requires certain number of MRs with certain number of radio interfaces to be placed at certain hops from gateway whereas TICA simply requires all MRs to have four data radios, does not require any careful router placement strategy and works with any placement of routers as verified by a comprehensive performance evaluation. In TICA, the mesh nodes do not use an $802.11 \mathrm{~b}$ channel for their control radios as in BFS-CA and hence, they are not affected by interference from other colocated IEEE $802.11 \mathrm{~b}$ wireless networks. If all the available frequency channels have already been assigned within the interference range of a link and a non-conflicting channel is not available, the BFS-CA scheme randomly selects a channel and assigns it to that link whereas TICA selects a LIC and assigns it to the link.

The most significant difference between TICA and existing CA schemes is that TICA uses topology control based on power control to build the topology for CA with the 
objective of minimizing the interference between the MRs whereas no other CA scheme has used topology control based on power control for CA.

Another significant difference between TICA and most other existing CA schemes is that TICA performs routing in addition to CA whereas most other CA schemes rely on routing protocols and complicated routing metrics for route selection.

Unlike TICA and D-HYA, other well-known CA schemes do not possess fault tolerance and have not provided any mechanism for recovery after a node failure.

Table 6.31: Features' Comparison of Related CA Schemes

\begin{tabular}{|c|c|c|c|c|c|c|}
\hline Feature & TICA & $\begin{array}{c}\text { C-HYA } \\
{[18]}\end{array}$ & $\begin{array}{c}\text { BFS-CA } \\
{[27]}\end{array}$ & $\begin{array}{c}\text { MesTiC } \\
\text { [21] }\end{array}$ & $\begin{array}{c}\text { D-HYA } \\
\text { [24] }\end{array}$ & $\begin{array}{l}\text { HMCP } \\
{[25]}\end{array}$ \\
\hline CA control & Centralized & Centralized & Centralized & Centralized & Distributed & Distributed \\
\hline $\begin{array}{l}\text { Knowledge of } \\
\text { traffic load }\end{array}$ & $\begin{array}{l}\text { Not } \\
\text { required }\end{array}$ & Required & $\begin{array}{l}\text { Not } \\
\text { required }\end{array}$ & Required & Required & $\begin{array}{l}\text { Not } \\
\text { required }\end{array}$ \\
\hline Channel switching & $\begin{array}{l}\text { Required for } \\
\text { failure } \\
\text { recovery }\end{array}$ & $\begin{array}{l}\text { Not } \\
\text { required }\end{array}$ & $\begin{array}{l}\text { Periodic } \\
\text { channel } \\
\text { switching } \\
\text { required }\end{array}$ & $\begin{array}{l}\text { Not } \\
\text { required }\end{array}$ & $\begin{array}{l}\text { Required as } \\
\text { CA changes } \\
\text { with traffic }\end{array}$ & $\begin{array}{l}\text { Per-packet } \\
\text { channel } \\
\text { switching } \\
\text { required }\end{array}$ \\
\hline Topology & $\begin{array}{l}\text { Topology } \\
\text { controlled } \\
\text { using TCA }\end{array}$ & Fixed & Fixed & Fixed & $\begin{array}{l}\text { Topology } \\
\text { defined by } \\
\text { spanning } \\
\text { tree }\end{array}$ & $\begin{array}{l}\text { Topology } \\
\text { changing } \\
\text { due to } \\
\text { channel } \\
\text { switching }\end{array}$ \\
\hline Power control & Yes & No & No & No & No & No \\
\hline Connectivity & $\begin{array}{l}\text { Ensured by } \\
\text { common } \\
\text { control } \\
\text { radio, } \\
\text { Ensured by } \\
\text { the CA } \\
\text { scheme } \\
\end{array}$ & $\begin{array}{l}\text { Ensured by } \\
\text { the CA } \\
\text { scheme }\end{array}$ & $\begin{array}{l}\text { Ensured by } \\
\text { common } \\
\text { control } \\
\text { radio }\end{array}$ & $\begin{array}{l}\text { Ensured by } \\
\text { common } \\
\text { control } \\
\text { radio }\end{array}$ & $\begin{array}{l}\text { Ensured by } \\
\text { the CA } \\
\text { scheme }\end{array}$ & $\begin{array}{l}\text { Ensured by } \\
\text { channel } \\
\text { switching }\end{array}$ \\
\hline Routing & $\begin{array}{l}\text { Performed } \\
\text { by the CA } \\
\text { scheme }\end{array}$ & No & No & No & $\begin{array}{l}\text { Performed } \\
\text { by the CA } \\
\text { scheme }\end{array}$ & No \\
\hline Failure recovery & Yes & No & No & No & Yes & No \\
\hline
\end{tabular}




\section{CHAPTER 7}

\section{CONCLUSION AND FUTURE WORK}

This chapter presents what has been accomplished in this thesis and what remains to be addressed in the future.

\subsection{Conclusions}

- A new topology control algorithm for multi-radio WMNs, Select $x$ for less than $x$, is proposed in this thesis. It controls the network topology by selecting the nearest neighbors for each node in the network. The proposed TCA not only minimizes the interference among the mesh routers but should also result in causing minimum interference to other co-located IEEE 802.11a-based wireless networks.

- A high average node degree indicates a stronger connectivity but results in a higher interference in the network. On the other hand, interference is less in the case of a lower average node degree, but it also affects the connectivity of the network and may result in a disconnected network. A high average node degree in the results for minimum power TR average node degree and minimum power IR average node degree for random and controlled random topologies in the case of Select 3 for less than 3 and Select 4 for less than 4 TCAs, indicates a stronger connectivity and leads to a connected network but also results in a higher interference in the network. On the other hand, a lower average node degree in case of Select 1 for less than 1 and Select 2 for less than 2 TCAs translates to less interference but also affects the connectivity of the network and results in a disconnected network. This indicates the trade-off between connectivity and interference. An adequate level of connectivity with low interference can be achieved by selecting the appropriate TCA.

- A new channel assignment algorithm for MRMC IEEE 802.11a-based WMNs, TICA, is proposed in this thesis which is based on topology control. As verified by a comprehensive performance evaluation, the improvement in the overall network throughput with TICA is significantly higher than the CCA scheme but is 
much higher than SRSC scheme in all three topologies. This is due to the fact that topology control based on power control results in an efficient frequency reuse during CA which leads to an overall improvement in the network throughput.

- The placement of the nodes and the length of links in the MPSPT affects the interference range and hence the channel assignment. In random and controlled random topologies, the random placement of nodes and the gateway results in variation in the length of links in the MPSPT. This can lead to LICs which may cause significant interference in the network and degrade the overall throughput, as indicated by the results for the average throughput of some seeds of random and controlled random topologies of a 36-node network.

- Due to the high degree of randomness in the location of nodes and gateway in the random topology, the resulting LICs cause significant interference and result in low throughput with TICA. Since the degree of randomness in the controlled random topology is relatively low, so the resulting LICs cause relatively less interference and result in a relatively better throughput with TICA. In the grid topology, there is no variation in the length of links in MPSPT which results in LICs with minimum interference and a high throughput with TICA, as shown by the results in Figures 6.9 and 6.10.

- For TICA, the maximum traffic that can reach the gateway depends on the number of links emanating from the gateway and the number of traffic sources using those links. So, the maximum traffic that can reach the gateway limits the actual throughput achieved by TICA as shown by the throughput analysis in Tables 6.16, 6.19, 6.22 and 6.26.

- It is observed that throughput achieved by CCA and SRSC schemes depends on the location of the gateway and traffic sources. In seeds of the random and controlled random topology where the gateway is located such that some traffic sources are located close to the gateway as compared to other traffic sources, the CCA and SRSC schemes exhibit a relatively high throughput. Since some traffic sources are located close to the gateway and outside the interference range of other traffic sources, so their traffic experiences less interference which leads to a relatively high throughput for these schemes. 
- The results show that Select 1 for less than 1 TCA always leads to a connected network in case of gird topology which is due to the symmetric nature of the grid topology.

- The results show that Select 1 for less than 1 TCA always leads to a disconnected network in case of random and controlled random topologies which is due to the random nature of these topologies. It is observed that Select 2 for less than 2 TCA leads to a disconnected network in case of random and controlled random topologies in most cases. However, in cases where it leads to a connected network for these topologies, the results show that the average throughput with Select 2 for less than 2 TCA is less than that with Select 3 for less than 3 TCA for TICA. This is due to the fact that Select 2 for less than 2 TCA results in a connectivity graph with low average node degree in TICA which leads to low throughput. Therefore, in case the Select 1 for less than 1 TCA leads to a disconnected network, this indicates that the topology is either random or controlled random and it is concluded that the next higher TCA for the gateway should be Select 3 for less than 3 in this case.

- As per the specification of the IEEE 802.11s standard for wireless mesh networks, the mesh routing protocol has to reside in the MAC layer. TICA not only performs channel assignment but performs routing as well by providing each node with the next hop information to reach the gateway node and hence, conforms to the requirements of IEEE $802.11 \mathrm{~s}$.

- The proposed CA algorithm, TICA, works with small-scale as well as large-scale networks. This is verified by the results of throughput comparison of TICA with other schemes for networks of 36 nodes and 144 nodes respectively. In comparison to CCA and SRSC schemes, the throughput improvement with TICA in a large-scale network of 144 nodes is significantly higher than the throughput improvement with TICA in a small-scale network of 36 nodes. The CCA and SRSC schemes perform poorly in 144-node network due to the large number of traffic sources. More traffic sources means more nodes competing for medium access. Increased contention for medium access combined with lack of power 
control in these schemes leads to more collisions and reduced throughput for these schemes.

- A centralized Failure Recovery Mechanism for TICA is proposed in this thesis which supports automatic and fast failure recovery. In case of node failure, after detecting the failed node, deleting the MPNT of the failed node and deleting it from the MPNT of its neighbors, the gateway runs the TCA and builds the MPSPT. Based on the new MPSPT, the gateway calculates the link rankings and the channel assignments. The new channel assignments are communicated to the mesh nodes by the gateway via a new CARM using the control radio over the control channel.

- TICA has shown a better performance as compared to BFS-CA and C-HYA schemes for similar scenarios. However, in these scenarios, it should be noted that the transmission range of mesh nodes in CCA and SRSC schemes is controlled by controlling either their transmission power or receiver sensitivity.

- The proposed CA algorithm, TICA, does not require modifications to the MAC protocol and therefore, can work with existing IEEE 802.11a-based interface hardware.

\subsection{Future Work}

Following are some aspects of this work which can be extended to improve the performance of the proposed algorithm.

- Since TICA uses the new approach of building the interference level for all the frequency channels, it can be enhanced to model and account for the interference from the co-located IEEE 802.11a-based wireless networks.

- Other algorithms for building the tree topology, such as Prim's algorithm [37] and Kruskal's algorithm [38], can be used for building the minimum power based tree which may lead to a further enhancement in the overall network throughput.

- The propagation model used is free-space model or two-ray model depending upon the cross-over distance. The performance of the proposed algorithm may be tested under more realistic propagation models such as Shadowing and Rayleighfading. 
- Other schemes for building the tree topology with the required node degree, such as the one proposed in [39], can be used which may lead to a better performance.

- The carrier sensing range is generally assumed to be twice the transmission range. However, carrier sensing range is a tunable parameter and an optimally tuned carrier sensing range can improve the network throughput in wireless mesh networks by enhancing the spatial frequency reuse and reducing collisions [40]. Therefore, the effects of carrier sensing range on the MAC layer performance in MRMC WMNs needs to be investigated and the performance of the proposed algorithm can be improved by controlling the carrier sensing range.

- The phenomenon of topology control based on power control impacts the pernode fairness of medium access based on CSMA/CA and hence the per-flow endto-end throughput fairness [41]. Therefore, the effects of power control on the per-flow end-to-end throughput fairness in multi-radio multi-channel wireless mesh networks needs to be investigated and the proposed algorithm can be extended to ensure per-node and hence per-flow fairness without sacrificing the gain in the overall network throughput.

- All the traffic in a WMN is directed towards the gateway. Depending on the number of links emanating from the gateway, the gateway has a fixed capacity which limits the maximum traffic that can reach the gateway. This gateway capacity is shared by all the traffic sources and throughput per traffic source is reduced as the number of traffic sources increases. Therefore, the traffic bottleneck at the gateway is the main reason of the capacity limitation of a WMN. The use of multiple gateways can increase the capacity of the WMN by preventing the formation of traffic bottlenecks [42]. Therefore, the capacity gains possible with the deployment of multiple gateways need to be investigated and the performance of the proposed algorithm can be improved by extending it for multiple gateways.

- The proposed algorithm can be extended for multiple gateways in a way such that each gateway is responsible for a subset of nodes in the network and the algorithmic computation and complexity is distributed among the gateways. 


\section{REFERENCES}

[1] N. Nandiraju, D. Nandiraju, L. Santhanam, B. He, J. Wang, and D.P. Agrawal, "Wireless Mesh Networks: Current Challenges and Future Directions of Web-in-theSky," IEEE Wireless Communications Magazine, Vol. 14(4), pp. 79-89, Aug. 2007.

[2] IEEE Standards Department, "Wireless LAN Medium Access Control (MAC) and Physical Layer (PHY) Specifications," IEEE Standard 802.11, Aug. 1999.

[3] W. Stallings, "IEEE 802.11: Wireless LANs from a to n," IT Professional, Vol. 6, Issue 5, pp. 32-37, Sept.-Oct., 2004.

[4] S. Misra, S. C. Misra, and I. Woungang, "Guide to Wireless Mesh Networks," Springer London, 2009.

[5] "IEEE Standard for Information Technology-Telecommunications and Information Exchange Between Systems-Local and Metropolitan Area Networks-Specific Requirements - Part 11: Wireless LAN MAC and PHY Specifications," IEEE Std 802.11-2007 (Revision of IEEE Std 802.11-1999), June 2007.

[6] IEEE 802.11s Task Group, Draft Amendment to Standard for Information Technology, Telecommunications and Information Exchange between Systems, "LAN/MAN Specific Requirements - Part 11: Wireless Medium Access Control (MAC) and Physical Layer (PHY) Specifications: Amendment: ESS Mesh Networking," IEEE P802.11s/D1.0, November 2006.

[7] M. J. Lee, J. Zheng, Y. Ko, and D. M. Shrestha, "Emerging Standards for Wireless Mesh Technology," IEEE Wireless Communications Magazine, Vol. 13(2), pp. 5663, April 2006.

[8] X. Wang and A. O. Lim, "IEEE 802.11s Wireless Mesh Networks: Framework and Challenges," Ad Hoc Networks, Vol. 6, No. 6, pp. 970-984, August 2008.

[9] J. D. Camp and E. W. Knightly, “The IEEE 802.11s Extended Service Set Mesh Networking Standard," IEEE Communications Magazine, Vol. 46, No. 8, pp. 120 126, Aug 2008.

[10] N. Li, J. Hou, and L. Sha, "Design and Analysis of an MST-Based Topology Control Algorithm," Proceedings of IEEE International Conference on Computer Communications, June 2003. 
[11] F. O. Aron, T. O. Olwal, A. Kurien, and Y. Hamam, "Network Preservation Through a Topology Control Algorithm for Wireless Mesh Networks," Proceedings of 2nd LASTED Africa Conference on Modelling and Simulation, Sep. 2008.

[12] V. Kawadia and P.R. Kumar, "Principles and Protocols for Power Control in Wireless Ad Hoc Networks", IEEE Journal on Selected Areas in Communications: Special Issues in Wireless Ad Hoc Networks, 2005.

[13] P. Gupta and P. R. Kumar, "Critical Power for Asymptotic Connectivity in Wireless Network," Stochastic Analysis, Control, Optimization and Applications: A Volume in Honor of W.H. Fleming, W. M. McEneany, G. Yin, and Q. Zhan, Eds. Birkhauser, Boston, pp. 547-566, 1998.

[14] P. Gupta and P. R. Kumar, "The Capacity of Wireless Networks," IEEE Transactions on Information Theory, Vol. IT-46, pp. 388-404, 2000.

[15] P. Santi, "Topology Control in Wireless Ad Hoc and Sensor Networks," $A C M$ Computing Survey, 37(2), pp. 164-194, 2005.

[16] S. Max and T. Wang, "Transmit Power Control in Wireless Mesh Networks Considered Harmful," Proceedings of Second International Conference on Advances in Mesh Networks, pp.73-78, 2009.

[17] Martin Kubisch, et al., "Distributed Algorithms for Transmission Power Control in Wireless Sensor Networks," Proceedings of IEEE Wireless Communications and Networking Conference, New Orleans, March 2003.

[18] A. Raniwala, K. Gopalan, and T. Chiueh, "Centralized Channel Assignment and Routing Algorithms for Multi-channel Wireless Mesh Networks," ACM Mobile Computing and Communications Review, Vol. 8(2), pp. 50-65, 2004.

[19] E. Hossain and K. Leung, "Wireless Mesh Networks: Architectures and Protocols," Springer US, 2007.

[20] R. Draves, J. Padhye, and B. Zill, "Routing in Multi-Radio, Multi-Hop Wireless Mesh Networks," Proceedings of ACM International Conference on Mobile Communications and Networking, 2004.

[21] H. Skalli, S. Ghosh, S. K. Das, L. Lenzini, and M. Conti, "Channel Assignment Strategies for Multiradio Wireless Mesh Networks: Issues and Solutions," IEEE 
Communications Magazine, Special Issue on "Wireless Mesh Networks”, pp. 86-93, Nov. 2007.

[22] P. Kyasanur, J. So, C. Chereddi, and N. Vaidya, "Multi-Channel Mesh Networks: Challenges and Protocols," IEEE Wireless Communications Magazine, Vol. 13(2), pp.30-36, April 2006.

[23] J. So and N. H. Vaidya, "Multi-Channel MAC for Ad Hoc Networks: Handling Multi-Channel Hidden Terminals Using a Single Transceiver," Proceedings of ACM International Symposium on Mobile Ad Hoc Networking and Computing, 2004.

[24] A. Raniwala and T.-C. Chiueh, "Architecture and Algorithms for an IEEE 802.11Based Multi-Channel Wireless Mesh Network," Proceedings of IEEE International Conference on Computer Communications, 2005.

[25] P. Kyasanur and N. Vaidya, "Routing and Interface Assignment in Multi-Channel Multi-Interface Wireless Networks," Proceedings of IEEE Wireless Communications and Networking Conference, New Orleans, LA, March 2005.

[26] P. Kyasanur and N. H. Vaidya, "Routing and Link-layer Protocols for MultiChannel Multi-Interface Ad Hoc Wireless Networks," ACM Mobile Computing and Communications Review, Vol. 10(1), pp. 31-43, Jan. 2006.

[27] K. Ramachandran, E. Belding, K. Almeroth, and M. Buddhikot, "InterferenceAware Channel Assignment in Multi-Radio W ireless Mesh Networks," Proceedings of IEEE International Conference on Computer Communications, 2006.

[28] K. Rosen, "Discrete Mathematics and its Applications," McGraw Hill, 1999.

[29] T. Rappaport, "Wireless Communications: Principles and Practice," 2nd Ed. Prentice Hall, Upper Saddle River, NJ, 2002.

[30] M. Mahdian, "On the Computational Complexity of Strong Edge Coloring," Discrete Applied Mathematics, Vol. 118, pp. 239-248, 2002.

[31] M. Shin, S. Lee, and Y.-A. Kim, "Distributed Channel Assignment for MultiRadio Wireless Networks," Proceedings of IEEE International Conference on Mobile Ad hoc and Sensor Systems, pp. 417-426, Oct. 8-11, 2006.

[32] E.W. Dijkstra, "A Note on Two Problems in Connection with Graphs," Numerische Mathematik, Vol. 1, pp. 269-271, 1959. 
[33] The VINT Project, "Network Simulator (NS) - version 2," available at http://www.isi.edu/nsnam/ns.

[34] The MathWorks Inc., "MATLAB® Reference Guide," Natick, MA, USA, 1992.

[35] R. A. Calvo and J. P. Campo, "Adding Multiple Interface Support in NS-2," University of Cantabria, Jan. 2007, available at http://personales.unican.es/aguerocr/files/ucMultilfacesSupport.pdf.

[36] Ad hoc On-Demand Distance Vector (AODV) Routing Protocol, RFC3561, available at http://www.ietf.org/rfc/rfc3561.txt.

[37] R. Prim, "Shortest Connection Networks and Some Generalizations," The Bell System Technical Journal, Vol. 36, pp. 1389-1401, 1957.

[38] J. Kruskal, "On the Shortest Spanning Tree for a Graph and Traveling Salesman Problem," Proceedings of American Mathematics Society, Vol. 7, pp. 48-50, 1956.

[39] S. C. Narula and C. A. Ho, "Degree-Constrained Minimum Spanning Tree," Computers and Operations Research, Vol. 7, pp. 239-249, 1980.

[40] J. Deng, B. Liang, and P. K. Varshney, "Tuning the Carrier Sensing Range of IEEE 802.11 MAC," Proceedings of IEEE Global Communications Conference, Dec. 2004.

[41] H. Y. Hsieh and R. Sivakumar, "Improving Fairness and Throughput in MultiHop Wireless Networks," Proceedings of IEEE International Conference on Networks, pp.569-578, 2001.

[42] S. Lakshmanan, R. Sivakumar, and K. Sundaresan, "Multi-Gateway Association in Wireless Mesh Networks," Ad Hoc Networks, Vol. 7(3), pp. 622-637, May 2009. 


\section{APPENDICES}

Appendix A. Final Neighbor Table (FNT)

A.1. FNTs from Select 1 for Less Than 1 TCA

Table A.1: FNTs of all nodes resulting from Select 1 for less than 1 TCA

\begin{tabular}{|c|c|c|c|c|c|c|c|}
\hline Node ID & $\begin{array}{l}\text { Neighbor } \\
\text { ID }\end{array}$ & $\begin{array}{c}\text { Distance } \\
\text { (m) }\end{array}$ & $\begin{array}{l}\text { Power } \\
\text { (mw) }\end{array}$ & Node ID & $\begin{array}{l}\text { Neighbor } \\
\text { ID }\end{array}$ & $\begin{array}{c}\text { Distance } \\
(\mathrm{m})\end{array}$ & $\begin{array}{l}\text { Power } \\
\text { (mW) }\end{array}$ \\
\hline 1 & 30 & 44 & 37 & 19 & 29 & 53 & 53 \\
\hline 2 & 23 & 94 & 166 & 20 & 33 & 51 & 49 \\
\hline 3 & 7 & 30 & 17 & 21 & 8 & 33 & 21 \\
\hline 3 & 11 & 49 & 45 & 22 & 6 & 52 & 51 \\
\hline 4 & 36 & 35 & 23 & 23 & 26 & 85 & 136 \\
\hline 5 & 30 & 125 & 293 & 23 & 2 & 94 & 166 \\
\hline 6 & 14 & 46 & 40 & 24 & 18 & 23 & 10 \\
\hline 6 & 22 & 52 & 51 & 25 & 32 & 28 & 15 \\
\hline 7 & 3 & 30 & 17 & 26 & 34 & 78 & 114 \\
\hline 8 & 21 & 33 & 21 & 26 & 23 & 85 & 136 \\
\hline 9 & 12 & 24 & 11 & 27 & 35 & 38 & 27 \\
\hline 10 & 34 & 40 & 30 & 28 & 13 & 53 & 53 \\
\hline 11 & 3 & 49 & 45 & 29 & 12 & 52 & 51 \\
\hline 11 & 33 & 49 & 45 & 29 & 19 & 53 & 53 \\
\hline 12 & 9 & 24 & 11 & 30 & 1 & 44 & 37 \\
\hline 12 & 29 & 52 & 51 & 30 & 5 & 125 & 293 \\
\hline 13 & 28 & 53 & 53 & 31 & 14 & 47 & 42 \\
\hline 13 & 16 & 66 & 82 & 32 & 25 & 28 & 15 \\
\hline 14 & 6 & 46 & 40 & 32 & 33 & 32 & 20 \\
\hline 14 & 31 & 47 & 42 & 33 & 32 & 32 & 20 \\
\hline 14 & 17 & 59 & 66 & 33 & 11 & 49 & 45 \\
\hline 15 & 18 & 42 & 33 & 33 & 20 & 51 & 49 \\
\hline 16 & 13 & 66 & 82 & 34 & 10 & 40 & 30 \\
\hline 17 & 14 & 59 & 66 & 34 & 26 & 78 & 114 \\
\hline 18 & 24 & 23 & 10 & 35 & 27 & 38 & 27 \\
\hline 18 & 15 & 42 & 33 & 36 & 4 & 35 & 23 \\
\hline
\end{tabular}


A.2. FNTs from Select 2 for Less Than 2 TCA

Table A.2: FNTs of all nodes resulting from Select 2 for less than 2 TCA

\begin{tabular}{|c|c|c|c|c|c|c|c|}
\hline Node ID & $\begin{array}{l}\text { Neighbor } \\
\text { ID }\end{array}$ & $\begin{array}{c}\begin{array}{c}\text { Distance } \\
(\mathrm{m})\end{array} \\
\end{array}$ & $\begin{array}{l}\text { Power } \\
\text { (mW) }\end{array}$ & Node ID & $\begin{array}{l}\text { Neighbor } \\
\text { ID }\end{array}$ & $\begin{array}{c}\text { Distance } \\
(\mathrm{m})\end{array}$ & $\begin{array}{l}\text { Power } \\
\text { (mW) }\end{array}$ \\
\hline 1 & 30 & 44 & 37 & 20 & 33 & 51 & 49 \\
\hline 1 & 7 & 103 & 199 & 20 & 32 & 56 & 59 \\
\hline 2 & 23 & 94 & 166 & 21 & 8 & 33 & 21 \\
\hline 2 & 16 & 106 & 211 & 21 & 15 & 81 & 123 \\
\hline 3 & 7 & 30 & 17 & 22 & 6 & 52 & 51 \\
\hline 3 & 11 & 49 & 45 & 22 & 29 & 81 & 123 \\
\hline 4 & 36 & 35 & 23 & 23 & 26 & 85 & 136 \\
\hline 4 & 35 & 53 & 53 & 23 & 2 & 94 & 166 \\
\hline 5 & 30 & 125 & 293 & 24 & 18 & 23 & 10 \\
\hline 5 & 27 & 126 & 297 & 24 & 15 & 46 & 40 \\
\hline 6 & 14 & 46 & 40 & 25 & 32 & 28 & 15 \\
\hline 6 & 22 & 52 & 51 & 25 & 33 & 46 & 40 \\
\hline 6 & 31 & 71 & 95 & 26 & 34 & 78 & 114 \\
\hline 7 & 3 & 30 & 17 & 26 & 10 & 81 & 123 \\
\hline 7 & 11 & 75 & 106 & 26 & 23 & 85 & 136 \\
\hline 7 & 1 & 103 & 199 & 27 & 35 & 38 & 27 \\
\hline 8 & 21 & 33 & 21 & 27 & 36 & 84 & 132 \\
\hline 8 & 15 & 51 & 49 & 27 & 5 & 126 & 297 \\
\hline 9 & 12 & 24 & 11 & 28 & 13 & 53 & 53 \\
\hline 9 & 29 & 57 & 61 & 28 & 36 & 101 & 191 \\
\hline 10 & 34 & 40 & 30 & 28 & 16 & 104 & 203 \\
\hline 10 & 26 & 81 & 123 & 29 & 12 & 52 & 51 \\
\hline 11 & 3 & 49 & 45 & 29 & 19 & 53 & 53 \\
\hline 11 & 33 & 49 & 45 & 29 & 9 & 57 & 61 \\
\hline 11 & 7 & 75 & 106 & 29 & 22 & 81 & 123 \\
\hline 11 & 19 & 88 & 145 & 30 & 1 & 44 & 37 \\
\hline 12 & 9 & 24 & 11 & 30 & 5 & 125 & 293 \\
\hline 12 & 29 & 52 & 51 & 31 & 14 & 47 & 42 \\
\hline 13 & 28 & 53 & 53 & 31 & 6 & 71 & 95 \\
\hline 13 & 16 & 66 & 82 & 31 & 17 & 83 & 129 \\
\hline 14 & 6 & 46 & 40 & 32 & 25 & 28 & 15 \\
\hline 14 & 31 & 47 & 42 & 32 & 33 & 32 & 20 \\
\hline 14 & 17 & 59 & 66 & 32 & 20 & 56 & 59 \\
\hline 15 & 18 & 42 & 33 & 33 & 32 & 32 & 20 \\
\hline 15 & 24 & 46 & 40 & 33 & 25 & 46 & 40 \\
\hline 15 & 8 & 51 & 49 & 33 & 11 & 49 & 45 \\
\hline 15 & 21 & 81 & 123 & 33 & 20 & 51 & 49 \\
\hline 16 & 13 & 66 & 82 & 34 & 10 & 40 & 30 \\
\hline 16 & 28 & 104 & 203 & 34 & 26 & 78 & 114 \\
\hline 16 & 2 & 106 & 211 & 35 & 27 & 38 & 27 \\
\hline 17 & 14 & 59 & 66 & 35 & 36 & 46 & 40 \\
\hline 17 & 31 & 83 & 129 & 35 & 4 & 53 & 53 \\
\hline 18 & 24 & 23 & 10 & 36 & 4 & 35 & 23 \\
\hline 18 & 15 & 42 & 33 & 36 & 35 & 46 & 40 \\
\hline
\end{tabular}




\begin{tabular}{|l|l|l|l|l|l|l|l|}
\hline 19 & 29 & 53 & 53 & 36 & 27 & 84 & 132 \\
\hline 19 & 11 & 88 & 145 & 36 & 28 & 101 & 191 \\
\hline
\end{tabular}

\section{A.3. FNTs from Select 3 for Less Than 3 TCA}

Table A.3: FNTs of all nodes resulting from Select 3 for less than 3 TCA

\begin{tabular}{|c|c|c|c|c|c|c|c|}
\hline Node ID & $\begin{array}{c}\text { Neighbor } \\
\text { ID }\end{array}$ & $\begin{array}{c}\text { Distance } \\
(\mathrm{m})\end{array}$ & $\begin{array}{l}\text { Power } \\
\text { (mW) }\end{array}$ & Node ID & $\begin{array}{l}\text { Neighbor } \\
\text { ID }\end{array}$ & $\begin{array}{c}\text { Distance } \\
(\mathrm{m})\end{array}$ & $\begin{array}{l}\text { Power } \\
\text { (mW) }\end{array}$ \\
\hline 1 & 30 & 44 & 37 & 19 & 29 & 53 & 53 \\
\hline 1 & 7 & 103 & 199 & 19 & 11 & 88 & 145 \\
\hline 1 & 25 & 122 & 279 & 19 & 9 & 93 & 162 \\
\hline 2 & 23 & 94 & 166 & 20 & 33 & 51 & 49 \\
\hline 2 & 16 & 106 & 211 & 20 & 32 & 56 & 59 \\
\hline 2 & 31 & 133 & 331 & 20 & 11 & 72 & 97 \\
\hline 3 & 7 & 30 & 17 & 21 & 8 & 33 & 21 \\
\hline 3 & 33 & 48 & 44 & 21 & 15 & 81 & 123 \\
\hline 3 & 11 & 49 & 45 & 21 & 18 & 93 & 162 \\
\hline 3 & 25 & 66 & 82 & 22 & 6 & 52 & 51 \\
\hline 4 & 36 & 35 & 23 & 22 & 29 & 81 & 123 \\
\hline 4 & 35 & 53 & 53 & 22 & 34 & 84 & 132 \\
\hline 4 & 27 & 85 & 136 & 22 & 23 & 124 & 288 \\
\hline 5 & 30 & 125 & 293 & 23 & 26 & 85 & 136 \\
\hline 5 & 27 & 126 & 297 & 23 & 2 & 94 & 166 \\
\hline 5 & 35 & 150 & 421 & 23 & 22 & 124 & 288 \\
\hline 6 & 14 & 46 & 40 & 24 & 18 & 23 & 10 \\
\hline 6 & 22 & 52 & 51 & 24 & 15 & 46 & 40 \\
\hline 6 & 12 & 58 & 63 & 24 & 8 & 90 & 152 \\
\hline 6 & 31 & 71 & 95 & 25 & 32 & 28 & 15 \\
\hline 7 & 3 & 30 & 17 & 25 & 33 & 46 & 40 \\
\hline 7 & 11 & 75 & 106 & 25 & 3 & 66 & 82 \\
\hline 7 & 33 & 76 & 108 & 25 & 1 & 122 & 279 \\
\hline 7 & 30 & 101 & 191 & 26 & 34 & 78 & 114 \\
\hline 7 & 1 & 103 & 199 & 26 & 10 & 81 & 123 \\
\hline 8 & 21 & 33 & 21 & 26 & 23 & 85 & 136 \\
\hline 8 & 15 & 51 & 49 & 27 & 35 & 38 & 27 \\
\hline 8 & 18 & 74 & 103 & 27 & 36 & 84 & 132 \\
\hline 8 & 24 & 90 & 152 & 27 & 4 & 85 & 136 \\
\hline 9 & 12 & 24 & 11 & 27 & 10 & 119 & 265 \\
\hline 9 & 29 & 57 & 61 & 27 & 5 & 126 & 297 \\
\hline 9 & 15 & 60 & 68 & 28 & 13 & 53 & 53 \\
\hline 9 & 19 & 93 & 162 & 28 & 36 & 101 & 191 \\
\hline 10 & 34 & 40 & 30 & 28 & 16 & 104 & 203 \\
\hline 10 & 26 & 81 & 123 & 29 & 12 & 52 & 51 \\
\hline 10 & 27 & 119 & 265 & 29 & 19 & 53 & 53 \\
\hline 11 & 3 & 49 & 45 & 29 & 9 & 57 & 61 \\
\hline 11 & 33 & 49 & 45 & 29 & 22 & 81 & 123 \\
\hline 11 & 20 & 72 & 97 & 30 & 1 & 44 & 37 \\
\hline 11 & 7 & 75 & 106 & 30 & 7 & 101 & 191 \\
\hline 11 & 19 & 88 & 145 & 30 & 5 & 125 & 293 \\
\hline
\end{tabular}




\begin{tabular}{|c|c|c|c|c|c|c|c|}
\hline 12 & 9 & 24 & 11 & 31 & 14 & 47 & 42 \\
\hline 12 & 29 & 52 & 51 & 31 & 6 & 71 & 95 \\
\hline 12 & 6 & 58 & 63 & 31 & 17 & 83 & 129 \\
\hline 12 & 17 & 100 & 187 & 31 & 2 & 133 & 331 \\
\hline 13 & 28 & 53 & 53 & 32 & 25 & 28 & 15 \\
\hline 13 & 16 & 66 & 82 & 32 & 33 & 32 & 20 \\
\hline 13 & 36 & 154 & 444 & 32 & 20 & 56 & 59 \\
\hline 14 & 6 & 46 & 40 & 33 & 32 & 32 & 20 \\
\hline 14 & 31 & 47 & 42 & 33 & 25 & 46 & 40 \\
\hline 14 & 17 & 59 & 66 & 33 & 3 & 48 & 44 \\
\hline 15 & 18 & 42 & 33 & 33 & 11 & 49 & 45 \\
\hline 15 & 24 & 46 & 40 & 33 & 20 & 51 & 49 \\
\hline 15 & 8 & 51 & 49 & 33 & 7 & 76 & 108 \\
\hline 15 & 9 & 60 & 68 & 34 & 10 & 40 & 30 \\
\hline 15 & 21 & 81 & 123 & 34 & 26 & 78 & 114 \\
\hline 16 & 13 & 66 & 82 & 34 & 22 & 84 & 132 \\
\hline 16 & 28 & 104 & 203 & 35 & 27 & 38 & 27 \\
\hline 16 & 2 & 106 & 211 & 35 & 36 & 46 & 40 \\
\hline 17 & 14 & 59 & 66 & 35 & 4 & 53 & 53 \\
\hline 17 & 31 & 83 & 129 & 35 & 5 & 150 & 421 \\
\hline 17 & 12 & 100 & 187 & 36 & 4 & 35 & 23 \\
\hline 18 & 24 & 23 & 10 & 36 & 35 & 46 & 40 \\
\hline 18 & 15 & 42 & 33 & 36 & 27 & 84 & 132 \\
\hline 18 & 8 & 74 & 103 & 36 & 28 & 101 & 191 \\
\hline 18 & 21 & 93 & 162 & 36 & 13 & 154 & 444 \\
\hline
\end{tabular}

\section{Appendix B. Failure Recovery Mechanism of TICA}

\section{B.1. Scenario 2 - Node 3 Fails}

In this scenario, Node 3 is supposed to have failed. After detecting its failure, the gateway deletes the MPNT of Node 3 and deletes Node 3 from the MPNT of its neighbors. The gateway runs the Select 3 for less than 3 TCA, builds the FNTs for all nodes and builds the connectivity graph as shown in Figure B.1. Note that the network resulting from Select 3 for less than 3 TCA is connected.

Since the connectivity graph resulting from Select 3 for less than 3 TCA is connected, the gateway builds the MPSPT with a MND of 4, as shown in Figure B.2.

Since the resulting MPSPT is connected, the gateway builds the link ranking for each link of the MPSPT, as shown in Table B.1. 


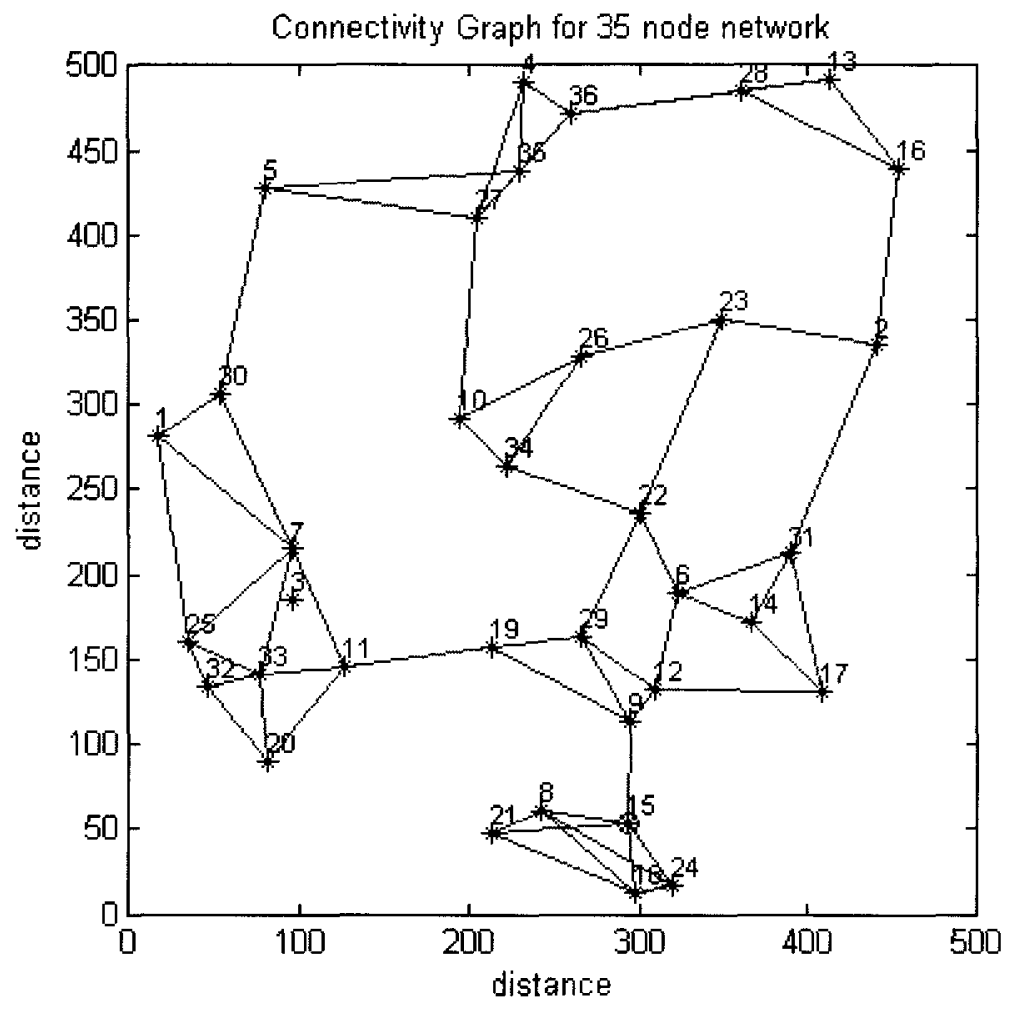

Figure B.1: Connectivity Graph with Select 3 for less than 3 TCA after Node 3 fails

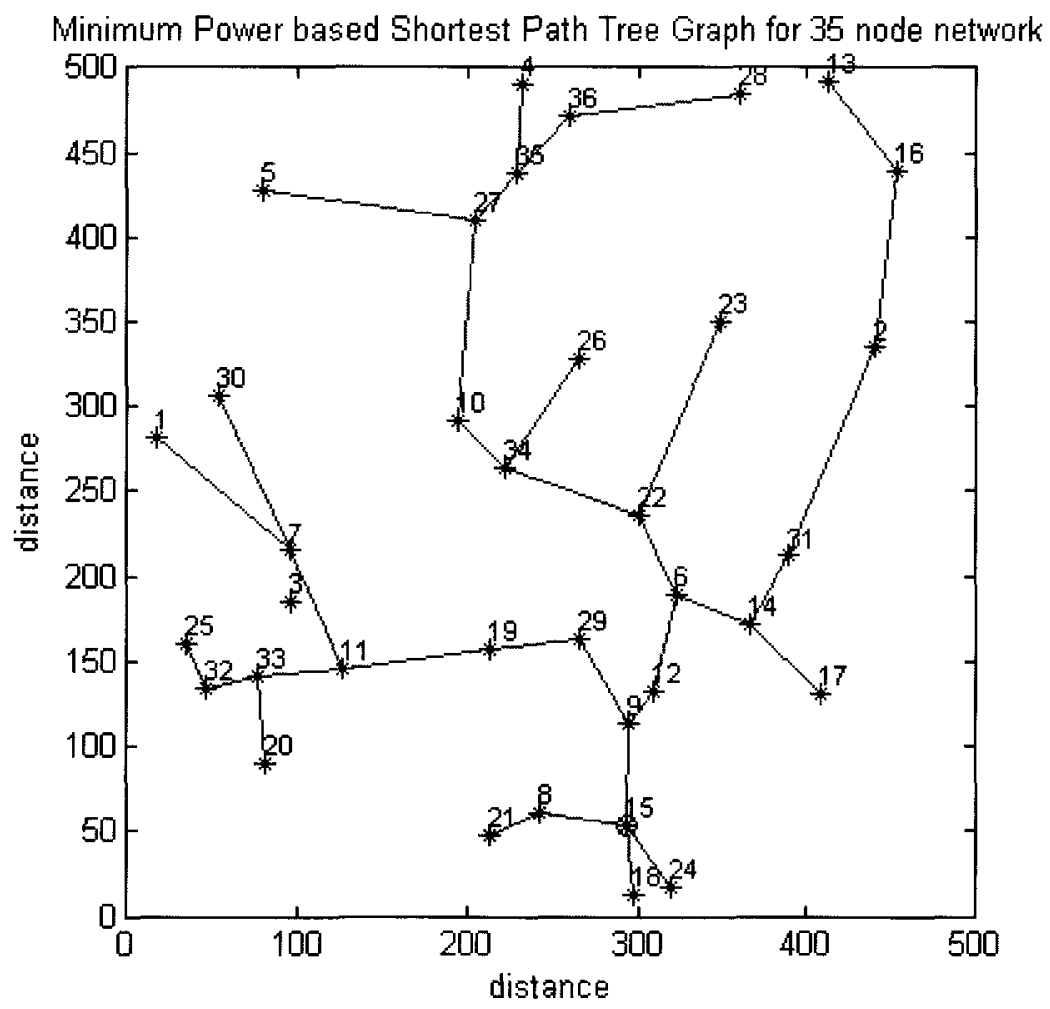

Figure B.2: MPSPT with a MND of 4 after Node 3 fails 
Table B.1: Link Ranking of MPSPT after Node 3 fails

\begin{tabular}{|c|c|c|c|c|c|}
\hline \multicolumn{2}{|c|}{ Link } & $\begin{array}{l}\text { Distance } \\
\text { (m) }\end{array}$ & $\begin{array}{l}\text { Power } \\
\text { (mW) }\end{array}$ & RANK & $\begin{array}{l}\text { Power from the farthest node of } \\
\text { the link to the gateway ( } \mathrm{mW} \text { ) }\end{array}$ \\
\hline 15 & 9 & 60 & 68 & 30 & 68 \\
\hline 9 & 12 & 24 & 11 & 19 & 79 \\
\hline 12 & 6 & 58 & 63 & 18 & 142 \\
\hline 6 & 22 & 52 & 51 & 11 & 193 \\
\hline 9 & 29 & 57 & 61 & 10 & 129 \\
\hline 29 & 19 & 53 & 53 & 9 & 182 \\
\hline 22 & 34 & 84 & 132 & 9 & 325 \\
\hline 19 & 11 & 88 & 145 & 8 & 327 \\
\hline 34 & 10 & 40 & 30 & 7 & 355 \\
\hline 6 & 14 & 46 & 40 & 6 & 182 \\
\hline 10 & 27 & 119 & 265 & 6 & 620 \\
\hline 14 & 31 & 47 & 42 & 4 & 224 \\
\hline 11 & 33 & 49 & 45 & 4 & 372 \\
\hline 27 & 35 & 38 & 27 & 4 & 647 \\
\hline 11 & 7 & 75 & 106 & 3 & 433 \\
\hline 31 & 2 & 133 & 331 & 3 & 555 \\
\hline 15 & 8 & 51 & 49 & 2 & 49 \\
\hline 33 & 32 & 32 & 20 & 2 & 392 \\
\hline 35 & 36 & 46 & 40 & 2 & 687 \\
\hline 2 & 16 & 106 & 211 & 2 & 766 \\
\hline 15 & 18 & 42 & 33 & 1 & 33 \\
\hline 15 & 24 & 46 & 40 & 1 & 40 \\
\hline 8 & 21 & 33 & 21 & 1 & 70 \\
\hline 14 & 17 & 59 & 66 & 1 & 248 \\
\hline 32 & 25 & 28 & 15 & 1 & 407 \\
\hline 33 & 20 & 51 & 49 & 1 & 421 \\
\hline 34 & 26 & 78 & 114 & 1 & 439 \\
\hline 22 & 23 & 124 & 288 & 1 & 481 \\
\hline 7 & 30 & 101 & 191 & 1 & 624 \\
\hline 7 & 1 & 103 & 199 & 1 & 632 \\
\hline 35 & 4 & 53 & 53 & 1 & 700 \\
\hline 16 & 13 & 66 & 82 & 1 & 848 \\
\hline 36 & 28 & 101 & 191 & 1 & 878 \\
\hline 27 & 5 & 126 & 297 & 1 & 917 \\
\hline
\end{tabular}

Based on the link ranking, the gateway assigns a channel to each link of the MPSPT, as shown in Table B.2. 
Table B.2: Channel Assignment for links of MPSPT after Node 3 fails

\begin{tabular}{|c|c|c|}
\hline Node & Neighbor & Channel \\
\hline 15 & 9 & 1 \\
\hline 9 & 12 & 2 \\
\hline 12 & 6 & 3 \\
\hline 6 & 22 & 4 \\
\hline 9 & 29 & 5 \\
\hline 29 & 19 & 6 \\
\hline 22 & 34 & 7 \\
\hline 19 & 11 & 8 \\
\hline 34 & 10 & 9 \\
\hline 6 & 14 & 10 \\
\hline 10 & 27 & 11 \\
\hline 14 & 31 & 11 \\
\hline 11 & 33 & 11 \\
\hline 27 & 35 & 10 \\
\hline 11 & 7 & 10 \\
\hline 31 & 2 & 8 \\
\hline 15 & 8 & 11 \\
\hline 33 & 32 & 9 \\
\hline 35 & 36 & 9 \\
\hline 2 & 16 & 6 \\
\hline 15 & 18 & 10 \\
\hline 15 & 24 & 9 \\
\hline 8 & 21 & 8 \\
\hline 14 & 17 & 9 \\
\hline 32 & 25 & 10 \\
\hline 33 & 20 & 7 \\
\hline 34 & 26 & 2 \\
\hline 22 & 23 & 8 \\
\hline 7 & 30 & 4 \\
\hline 7 & 1 & 3 \\
\hline 35 & 4 & 8 \\
\hline 16 & 13 & 11 \\
\hline 36 & 28 & 7 \\
\hline 27 & 5 & 5 \\
\hline
\end{tabular}

Before its failure, Node 3 was part of the $11^{\text {th }}$-ranked link. As shown in Table B.1, the link rankings and hence the channel assignment of all links from the $4^{\text {th }}$-ranked link and onwards, change after the failure of Node 3. 


\section{B.2. Scenario 3 - Node 30 Fails}

In this scenario, Node 30 fails. After detecting its failure, the gateway deletes the MPNT of Node 30 and deletes Node 30 from the MPNT of its neighbors. The gateway runs the Select 3 for less than 3 TCA, builds the FNTs for all nodes and builds the connectivity graph as shown in Figure B.3. Note that the network resulting from Select 3 for less than 3 TCA is connected.

Since the connectivity graph resulting from Select 3 for less than 3 TCA is connected, the gateway builds the MPSPT with a MND of 4, as shown in Figure B.4.

Since the resulting MPSPT is connected, the gateway builds the link ranking for each link of the MPSPT, as shown in Table B.3.

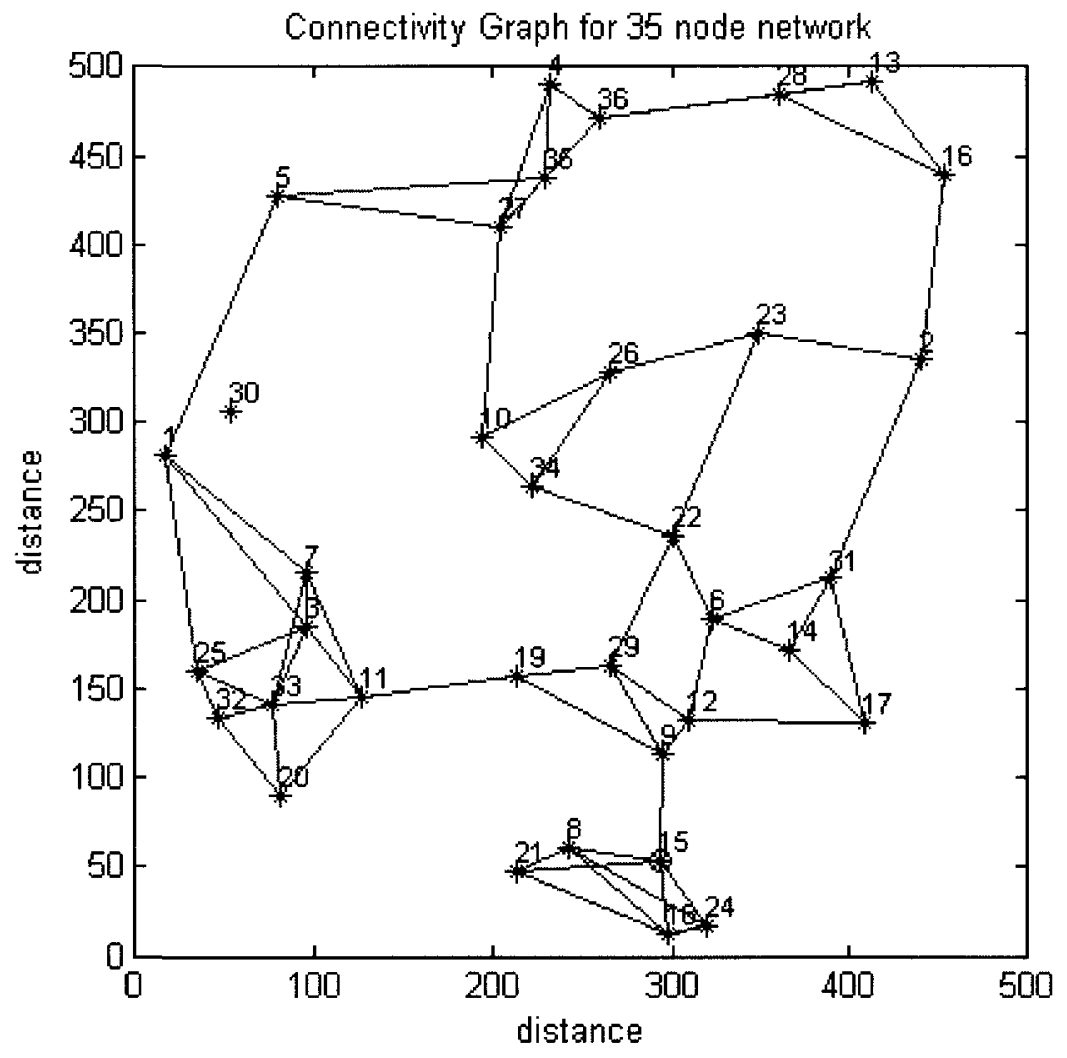

Figure B.3: Connectivity Graph with Select 3 for less than 3 TCA after Node 30 fails 


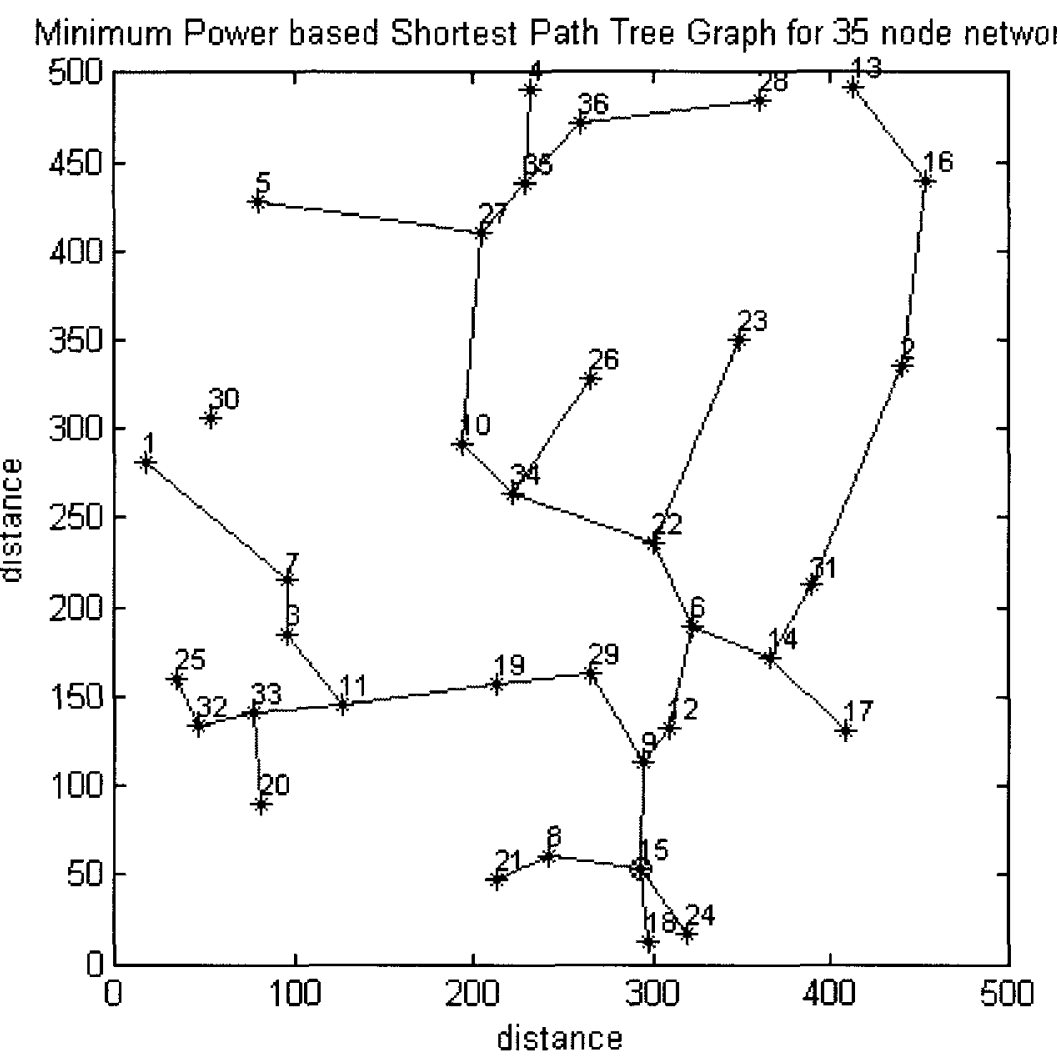

Figure B.4: MPSPT with a MND of 4 after Node 30 fails

Table B.3: Link Ranking of MPSPT after Node 30 fails

\begin{tabular}{|c|c|c|c|c|c|}
\hline \multicolumn{2}{|c|}{ Link } & $\begin{array}{c}\text { Distance } \\
(\mathbf{m})\end{array}$ & $\begin{array}{c}\text { Power } \\
(\mathbf{m W})\end{array}$ & RANK & $\begin{array}{c}\text { Power from the farthest node of } \\
\text { the link to the gateway (mW) }\end{array}$ \\
\hline 15 & 9 & 60 & 68 & 30 & 68 \\
\hline 9 & 12 & 24 & 11 & 19 & 142 \\
\hline 12 & 6 & 58 & 63 & 18 & 193 \\
\hline 6 & 22 & 52 & 51 & 11 & 129 \\
\hline 9 & 29 & 57 & 61 & 10 & 182 \\
\hline 29 & 19 & 53 & 53 & 9 & 325 \\
\hline 22 & 34 & 84 & 132 & 9 & 327 \\
\hline 19 & 11 & 88 & 145 & 8 & 355 \\
\hline 34 & 10 & 40 & 30 & 7 & 182 \\
\hline 6 & 14 & 46 & 40 & 6 & 620 \\
\hline 10 & 27 & 119 & 265 & 6 & 224 \\
\hline 14 & 31 & 47 & 42 & 4 & 372 \\
\hline 11 & 33 & 49 & 45 & 4 & 647 \\
\hline 27 & 35 & 38 & 27 & 4 & 372 \\
\hline 11 & 3 & 49 & 45 & 3 & 555 \\
\hline 31 & 2 & 133 & 331 & 3 & 49 \\
\hline 15 & 8 & 51 & 49 & 2 & 389 \\
\hline 3 & 7 & 30 & 17 & 2 & \\
\hline & & & & & \\
\hline
\end{tabular}




\begin{tabular}{|c|c|c|c|c|c|}
\hline 33 & 32 & 32 & 20 & 2 & 392 \\
\hline 35 & 36 & 46 & 40 & 2 & 687 \\
\hline 2 & 16 & 106 & 211 & 2 & 766 \\
\hline 15 & 18 & 42 & 33 & 1 & 33 \\
\hline 15 & 24 & 46 & 40 & 1 & 40 \\
\hline 8 & 21 & 33 & 21 & 1 & 70 \\
\hline 14 & 17 & 59 & 66 & 1 & 248 \\
\hline 32 & 25 & 28 & 15 & 1 & 407 \\
\hline 33 & 20 & 51 & 49 & 1 & 421 \\
\hline 34 & 26 & 78 & 114 & 1 & 439 \\
\hline 22 & 23 & 124 & 288 & 1 & 481 \\
\hline 7 & 1 & 103 & 199 & 1 & 588 \\
\hline 35 & 4 & 53 & 53 & 1 & 700 \\
\hline 16 & 13 & 66 & 82 & 1 & 848 \\
\hline 36 & 28 & 101 & 191 & 1 & 878 \\
\hline 27 & 5 & 126 & 297 & 1 & 917 \\
\hline
\end{tabular}

Based on the link ranking, the gateway assigns a channel to each link of the MPSPT, as shown in Table B.4.

Table B.4: Channel Assignment for links of MPSPT after Node 30 fails

\begin{tabular}{|c|c|c||c|c|c|}
\hline Node & Neighbor & Channel & Node & Neighbor & Channel \\
\hline 15 & 9 & 1 & 3 & 7 & 9 \\
\hline 9 & 12 & 2 & 33 & 32 & 7 \\
\hline 12 & 6 & 3 & 35 & 36 & 9 \\
\hline 6 & 22 & 4 & 2 & 16 & 6 \\
\hline 9 & 29 & 5 & 15 & 18 & 10 \\
\hline 29 & 19 & 6 & 15 & 24 & 9 \\
\hline 22 & 34 & 7 & 8 & 21 & 8 \\
\hline 19 & 11 & 8 & 14 & 17 & 9 \\
\hline 34 & 10 & 9 & 32 & 25 & 10 \\
\hline 6 & 14 & 10 & 33 & 20 & 6 \\
\hline 10 & 27 & 11 & 34 & 26 & 2 \\
\hline 14 & 31 & 11 & 22 & 23 & 8 \\
\hline 11 & 33 & 11 & 7 & 1 & 3 \\
\hline 27 & 35 & 10 & 35 & 4 & 8 \\
\hline 11 & 3 & 10 & 16 & 13 & 11 \\
\hline 31 & 2 & 8 & 36 & 28 & 7 \\
\hline 15 & 8 & 11 & 27 & 5 & 5 \\
\hline
\end{tabular}

Before its failure, Node 30 was part of the $20^{\text {th }}$-ranked link. As shown in Table B.3, the link rankings and hence the channel assignment of all links from the $4^{\text {th }}$-ranked link and onwards, change after the failure of Node 30. 


\section{B.3. Scenario 4 - Node 5 Fails}

In this scenario, I suppose that Node 5 fails. After detecting its failure, the gateway deletes the MPNT of Node 5 and deletes Node 5 from the MPNT of its neighbors. The gateway runs the Select 3 for less than 3 TCA, builds the FNTs for all nodes and builds the connectivity graph as shown in Figure B.5. Note that the network resulting from Select 3 for less than 3 TCA is connected.

Since the connectivity graph resulting from Select 3 for less than 3 TCA is connected, the gateway builds the MPSPT with a MND of 4, as shown in Figure B.6.

Since the resulting MPSPT is connected, the gateway builds the link ranking for each link of the MPSPT, as shown in Table B.5.

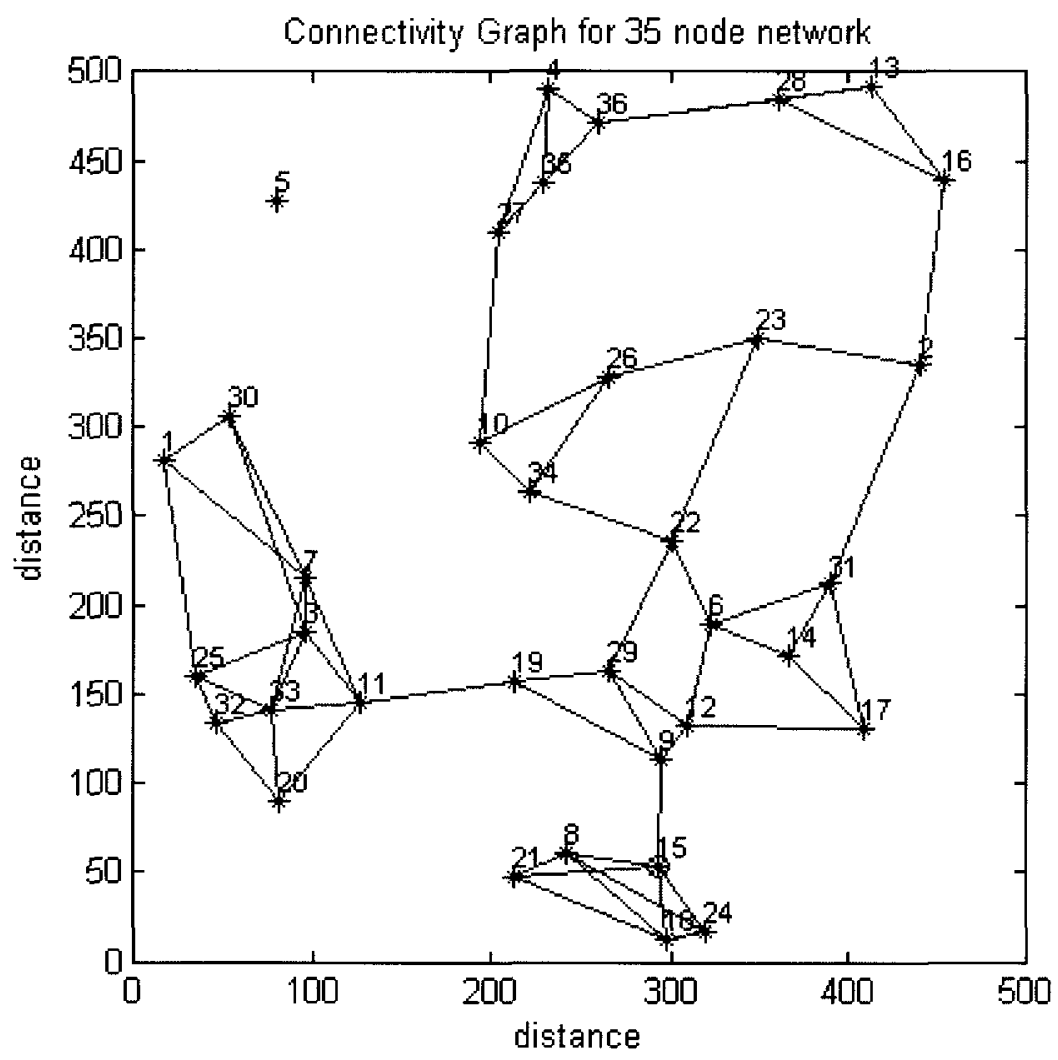

Figure B.5: Connectivity Graph with Select 3 for less than 3 TCA after Node 5 fails 


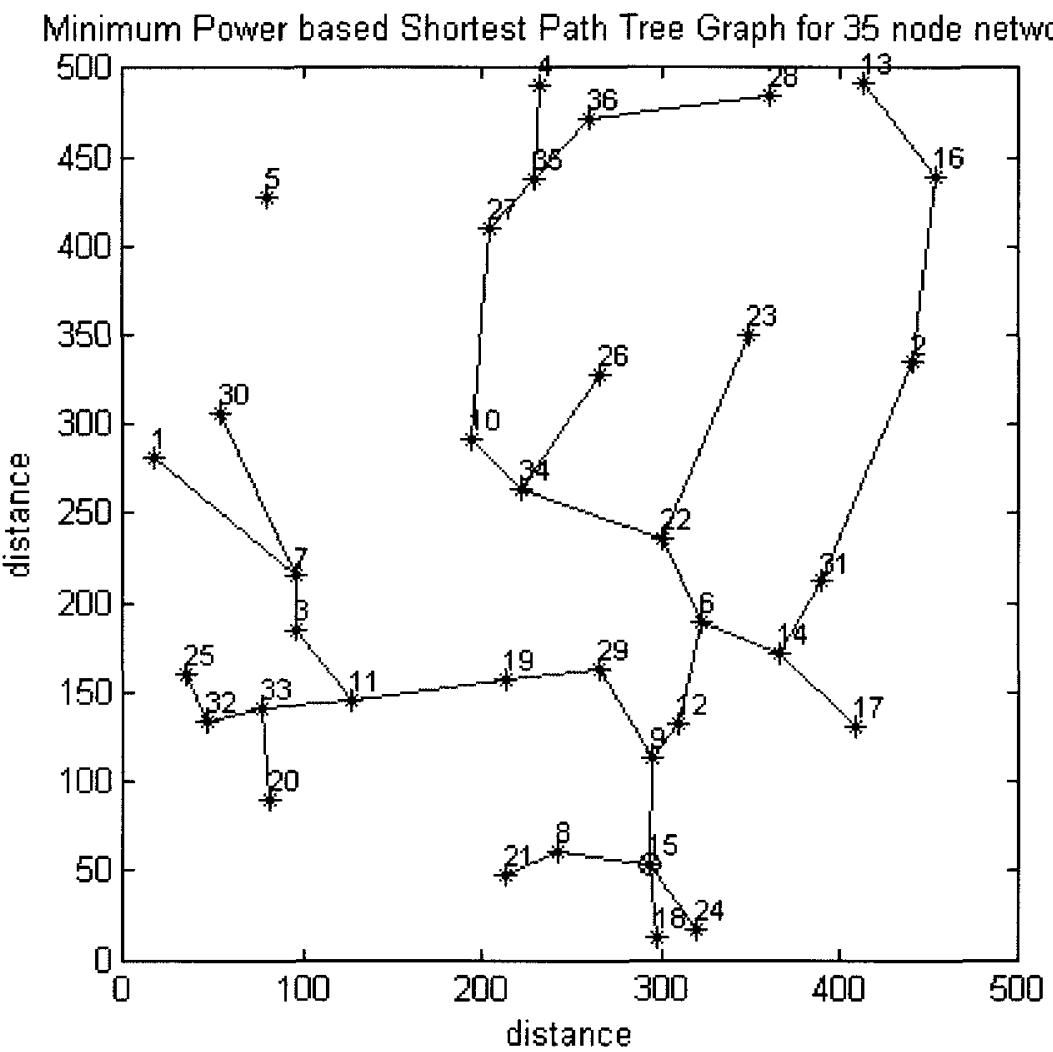

Figure B.6: MPSPT with a MND of 4 after Node 5 fails

Table B.5: Link Ranking of MPSPT after Node 5 fails

\begin{tabular}{|c|c|c|c|c|c|}
\hline \multicolumn{2}{|c|}{ Link } & $\begin{array}{c}\text { Distance } \\
(\mathbf{m})\end{array}$ & $\begin{array}{c}\text { Power } \\
(\mathbf{m W})\end{array}$ & RANK & $\begin{array}{c}\text { Power from the farthest node of } \\
\text { the link to the gateway (mW) }\end{array}$ \\
\hline 15 & 9 & 60 & 68 & 30 & 68 \\
\hline 9 & 12 & 24 & 11 & 18 & 142 \\
\hline 12 & 6 & 58 & 63 & 17 & 129 \\
\hline 9 & 29 & 57 & 61 & 11 & 182 \\
\hline 29 & 19 & 53 & 53 & 10 & 193 \\
\hline 6 & 22 & 52 & 51 & 10 & 327 \\
\hline 19 & 11 & 88 & 145 & 9 & 325 \\
\hline 22 & 34 & 84 & 132 & 8 & 182 \\
\hline 6 & 14 & 46 & 40 & 6 & 355 \\
\hline 34 & 10 & 40 & 30 & 6 & 620 \\
\hline 10 & 27 & 119 & 265 & 5 & 224 \\
\hline 14 & 31 & 47 & 42 & 4 & 372 \\
\hline 11 & 3 & 49 & 45 & 4 & 372 \\
\hline 11 & 33 & 49 & 45 & 4 & 647 \\
\hline 27 & 35 & 38 & 27 & 4 & 389 \\
\hline 3 & 7 & 30 & 17 & 3 & 555 \\
\hline 31 & 2 & 133 & 331 & 3 & 49 \\
\hline 15 & 8 & 51 & 49 & 2 & 79 \\
\hline
\end{tabular}




\begin{tabular}{|c|c|c|c|c|c|}
\hline 33 & 32 & 32 & 20 & 2 & 392 \\
\hline 35 & 36 & 46 & 40 & 2 & 687 \\
\hline 2 & 16 & 106 & 211 & 2 & 766 \\
\hline 15 & 18 & 42 & 33 & 1 & 33 \\
\hline 15 & 24 & 46 & 40 & 1 & 40 \\
\hline 8 & 21 & 33 & 21 & 1 & 70 \\
\hline 14 & 17 & 59 & 66 & 1 & 248 \\
\hline 32 & 25 & 28 & 15 & 1 & 407 \\
\hline 33 & 20 & 51 & 49 & 1 & 421 \\
\hline 34 & 26 & 78 & 114 & 1 & 439 \\
\hline 22 & 23 & 124 & 288 & 1 & 481 \\
\hline 7 & 30 & 101 & 191 & 1 & 580 \\
\hline 7 & 1 & 103 & 199 & 1 & 588 \\
\hline 35 & 4 & 53 & 53 & 1 & 700 \\
\hline 16 & 13 & 66 & 82 & 1 & 848 \\
\hline 36 & 28 & 101 & 191 & 1 & 878 \\
\hline
\end{tabular}

Based on the link ranking, the gateway assigns a channel to each link of the MPSPT, as shown in Table B.6.

Table B.6: Channel Assignment for links of MPSPT after Node 5 fails

\begin{tabular}{|c|c|c|c|c|c|}
\hline Node & Neighbor & Channel & Node & Neighbor & Channel \\
\hline 15 & 9 & 1 & 15 & 8 & 11 \\
\hline 9 & 12 & 2 & 33 & 32 & 8 \\
\hline 12 & 6 & 3 & 35 & 36 & 9 \\
\hline 9 & 29 & 4 & 2 & 16 & 10 \\
\hline 29 & 19 & 5 & 15 & 18 & 10 \\
\hline 6 & 22 & 6 & 15 & 24 & 9 \\
\hline 19 & 11 & 7 & 8 & 21 & 8 \\
\hline 22 & 34 & 8 & 14 & 17 & 10 \\
\hline 6 & 14 & 9 & 32 & 25 & 11 \\
\hline 34 & 10 & 10 & 33 & 20 & 6 \\
\hline 10 & 27 & 11 & 34 & 26 & 2 \\
\hline 14 & 31 & 11 & 22 & 23 & 7 \\
\hline 11 & 3 & 11 & 7 & 30 & 3 \\
\hline 11 & 33 & 10 & 7 & 1 & 1 \\
\hline 27 & 35 & 10 & 35 & 4 & 8 \\
\hline 3 & 7 & 9 & 16 & 13 & 11 \\
\hline 31 & 2 & 7 & 36 & 28 & 6 \\
\hline
\end{tabular}

Before its failure, Node 5 was part of the $34^{\text {th }}$-ranked link. As shown in Table B.5, the link rankings and hence the channel assignment of all links from the $11^{\text {th }}$-ranked link and onwards, change after the failure of Node 5. 


\section{Appendix C. MPSPT}

\section{C.1. MPSPT for Random Topology - 36-node Network}

\section{C.1.1. Seed 2}

For the $2^{\text {nd }}$-random topology, the MPSPT with a MND of 4 resulting from Select 3 for less than 3 TCA for a 36-node network is shown in Figure C.1.

\section{C.1.2. Seed 3}

For the $3^{\text {rd }}$-random topology, the MPSPT with a MND of 4 resulting from Select 3 for less than 3 TCA for a 36 node-network is shown in Figure C.2.

\section{C.1.3. Seed 4}

For the $4^{\text {th }}$-random topology, the MPSPT with a MND of 4 resulting from Select 3 for less than 3 TCA for a 36-node network is shown in Figure C.3.

\section{C.1.4. Seed 5}

For the $5^{\text {th }}$-random topology, the MPSPT with a MND of 4 resulting from Select 3 for less than 3 TCA for a 36-node network is shown in Figure C.4.

\section{C.1.5. Seed 6}

For the $6^{\text {th }}$-random topology, the MPSPT with a MND of 4 resulting from Select 3 for less than 3 TCA for a 36-node network is shown in Figure C.5.

\section{C.1.6. Seed 7}

For the $7^{\text {th }}$-random topology, the MPSPT with a MND of 4 resulting from Select 3 for less than 3 TCA for a 36-node network is shown in Figure C.6.

\section{C.1.7. Seed 8}

For the $8^{\text {th }}$-random topology, the MPSPT with a MND of 4 resulting from Select 3 for less than 3 TCA for a 36-node network is shown in Figure C.7.

\section{C.1.8. Seed 9}

For the $9^{\text {th }}$-random topology, the MPSPT with a MND of 4 resulting from Select 3 for less than 3 TCA for a 36-node network is shown in Figure C.8.

\section{C.1.9. Seed 10}

For the $10^{\text {th }}$-random topology, the MPSPT with a MND of 4 resulting from Select 3 for less than 3 TCA for a 36-node network is shown in Figure C.9. 


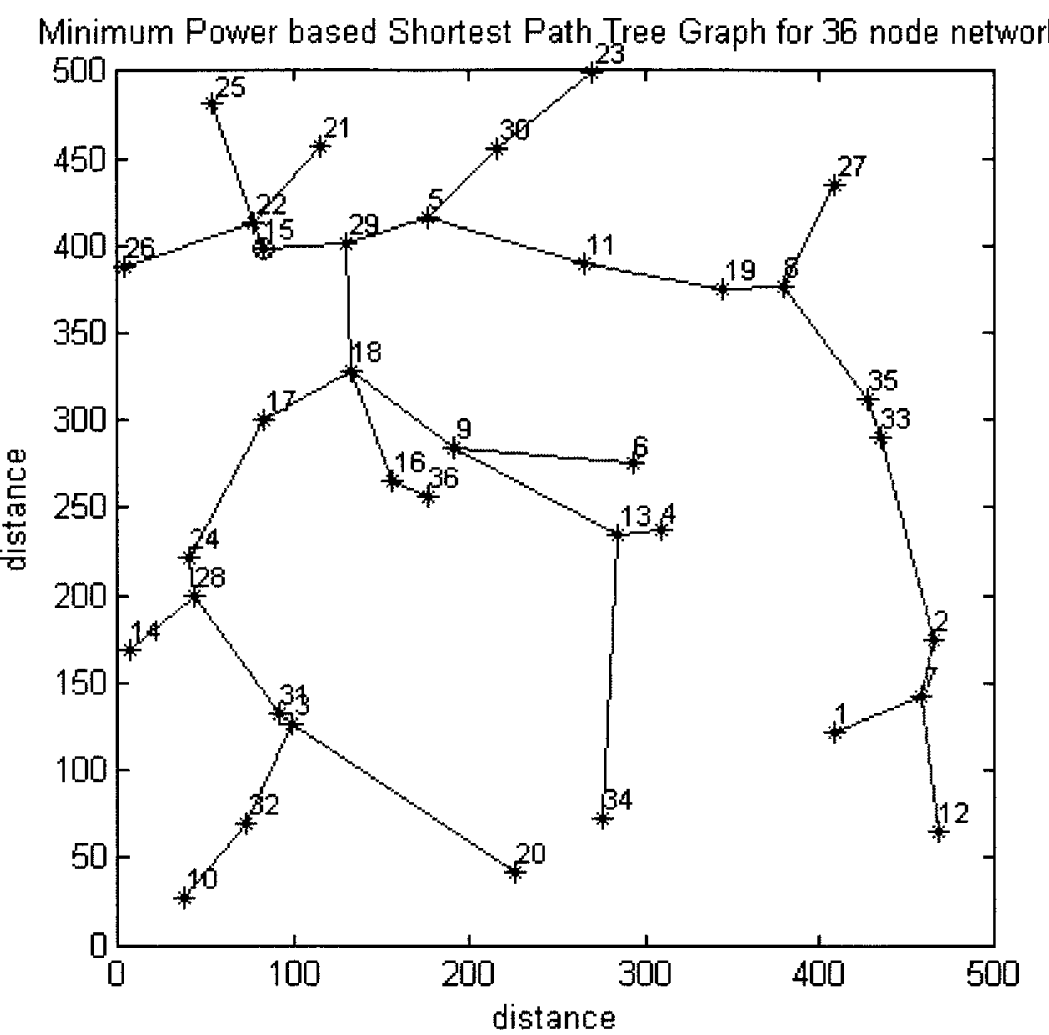

Figure C.1: MPSPT for $2^{\text {nd }}$ RT

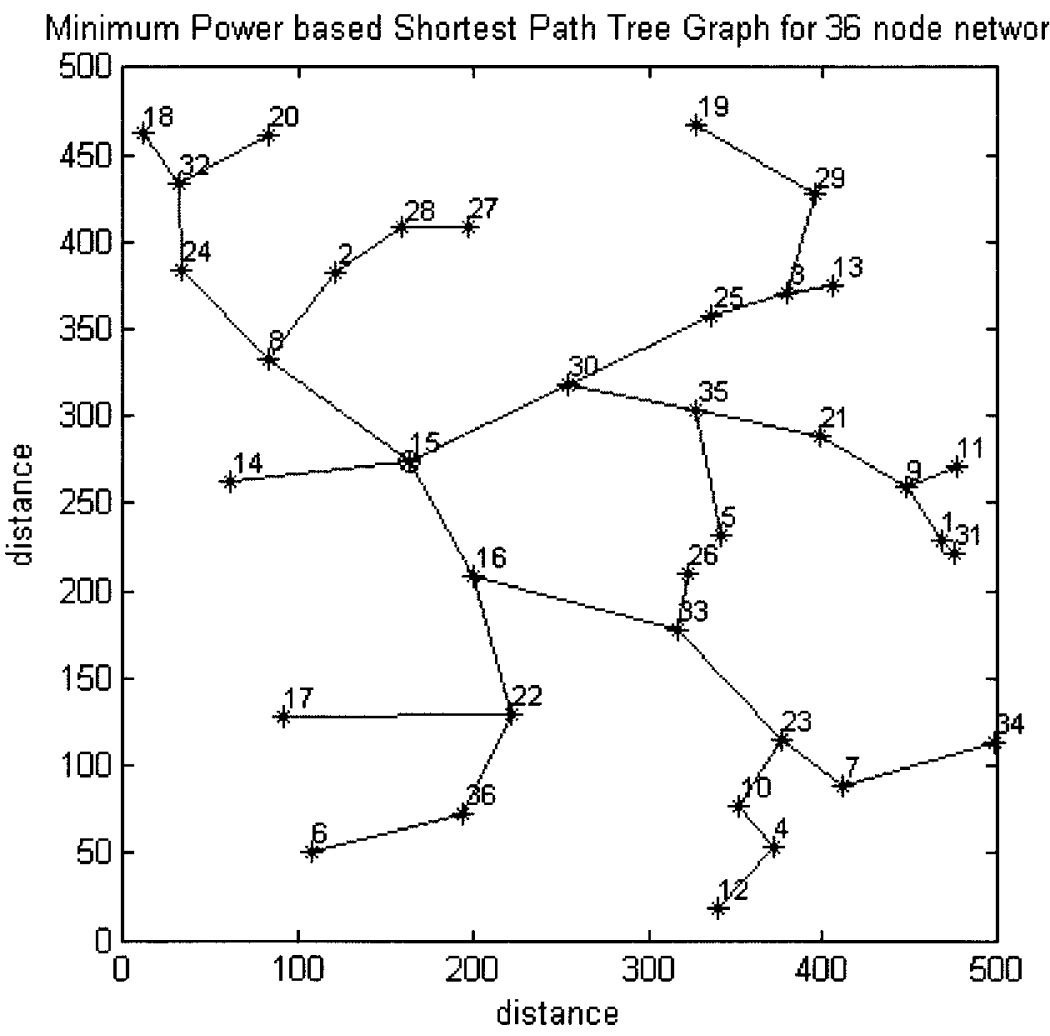

Figure C.2: MPSPT for $3^{\text {rd }}$ RT 


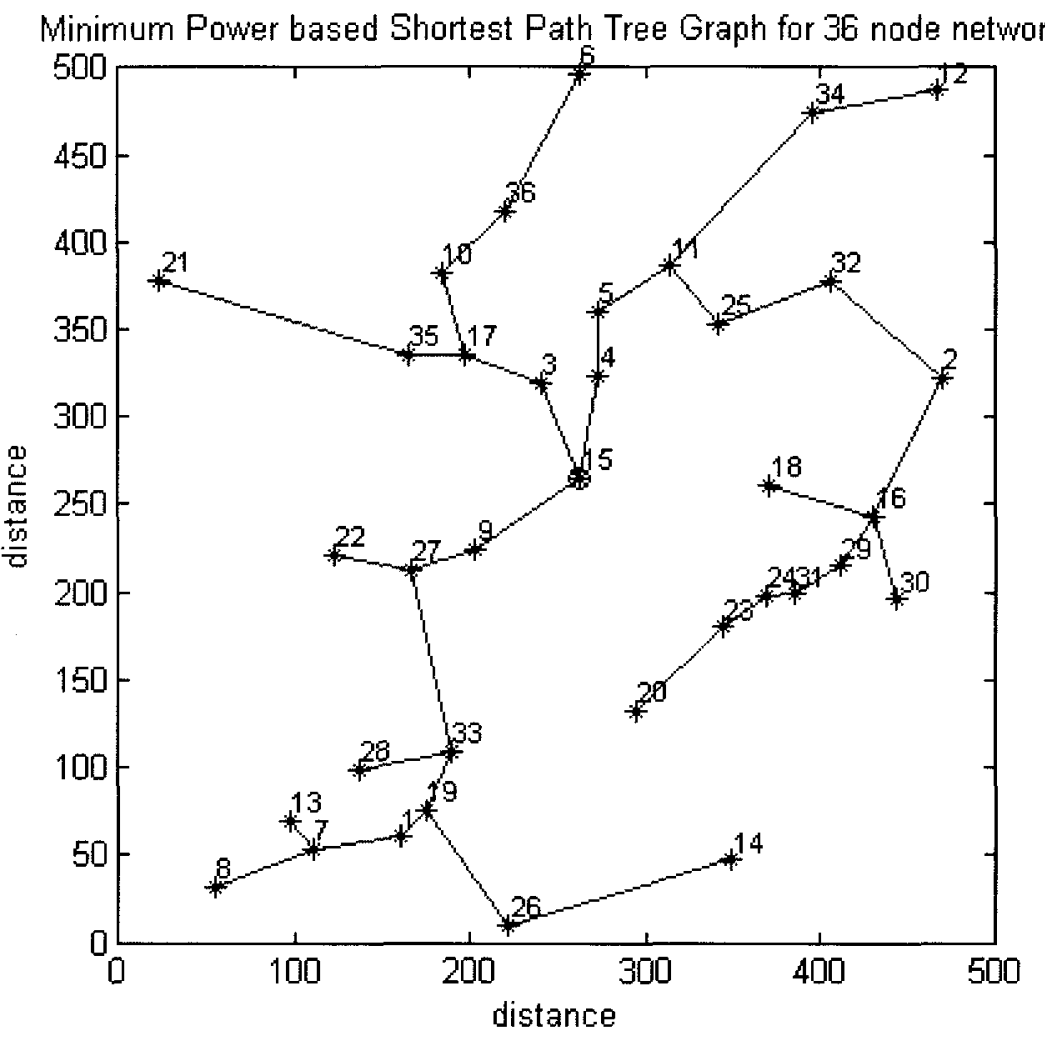

Figure C.3: MPSPT for $4^{\text {th }}$ RT

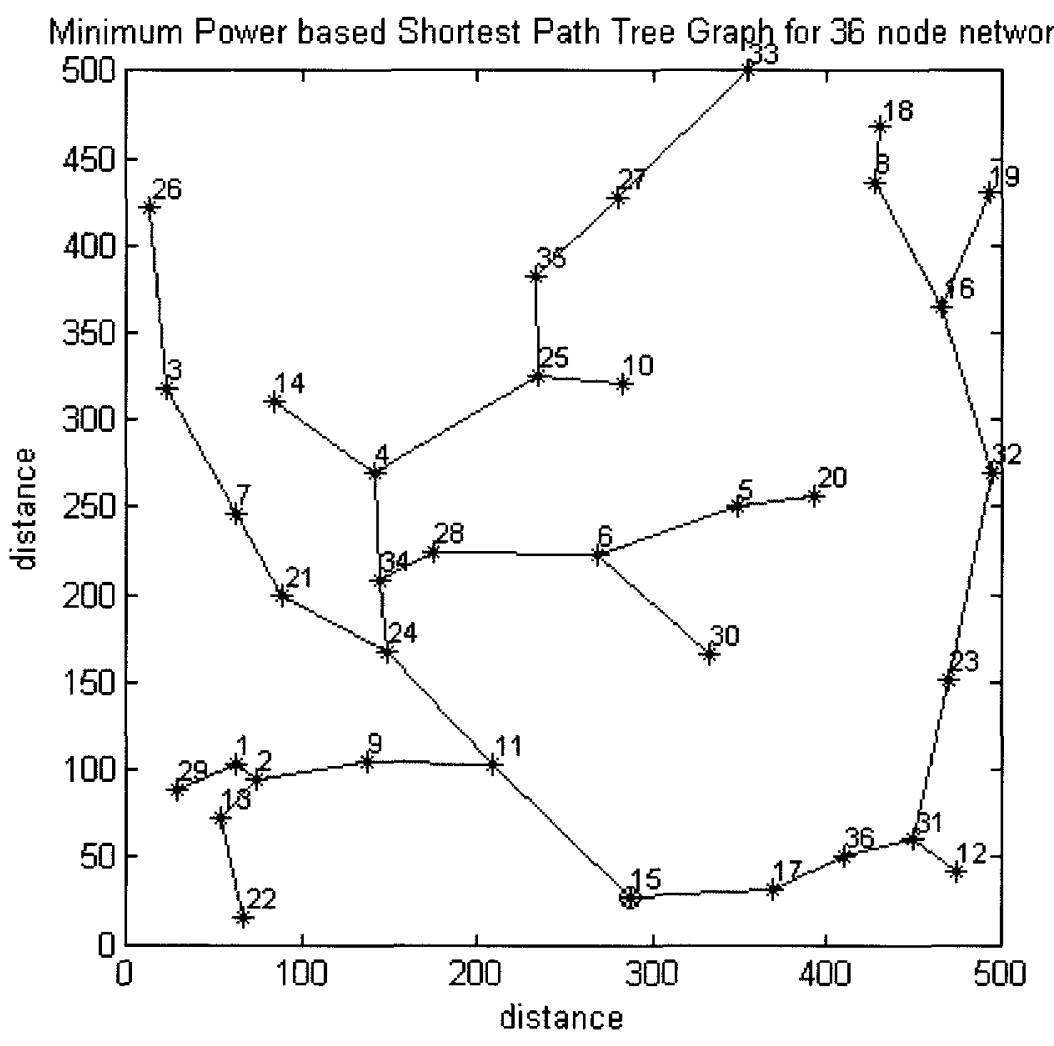

Figure C.4: MPSPT for $5^{\text {th }}$ RT 


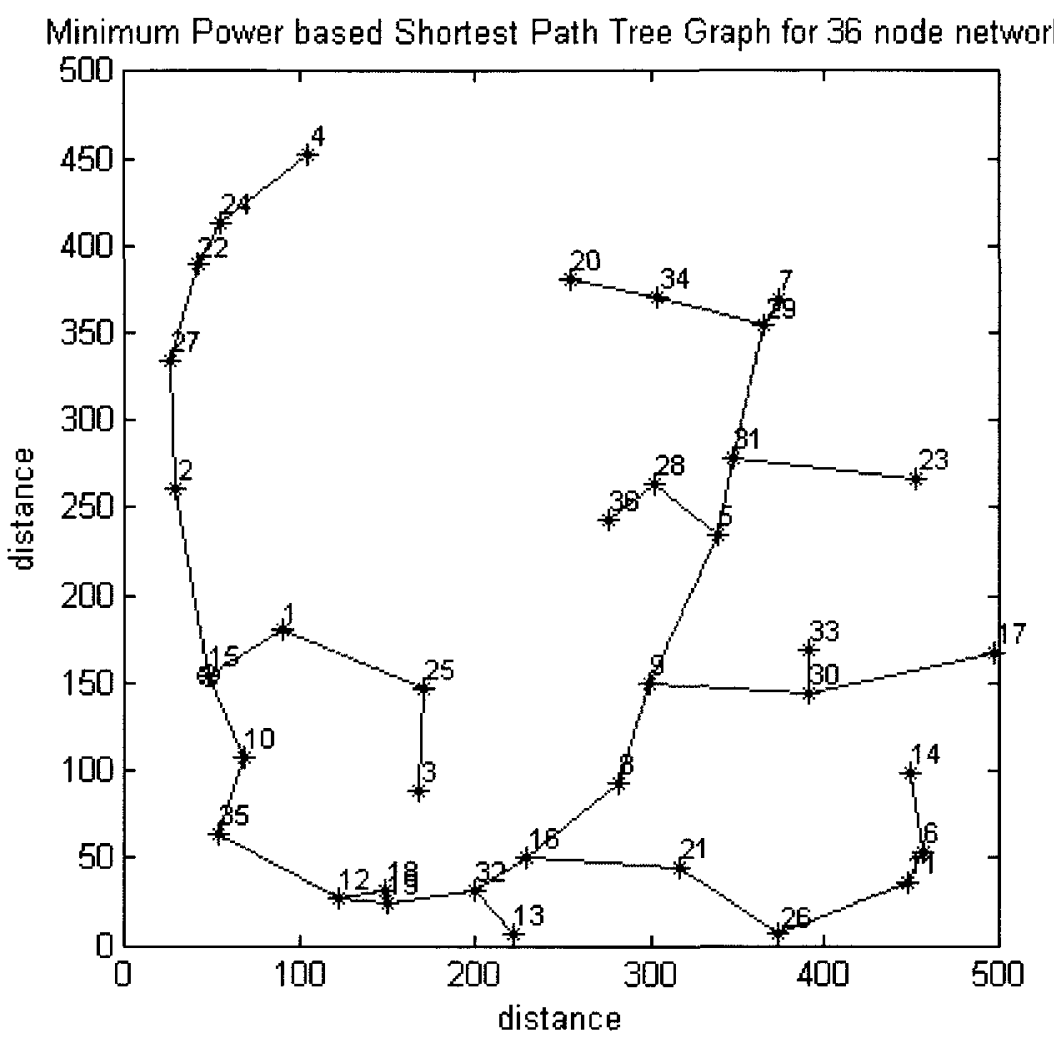

Figure C.5: MPSPT for $6^{\text {th }}$ RT

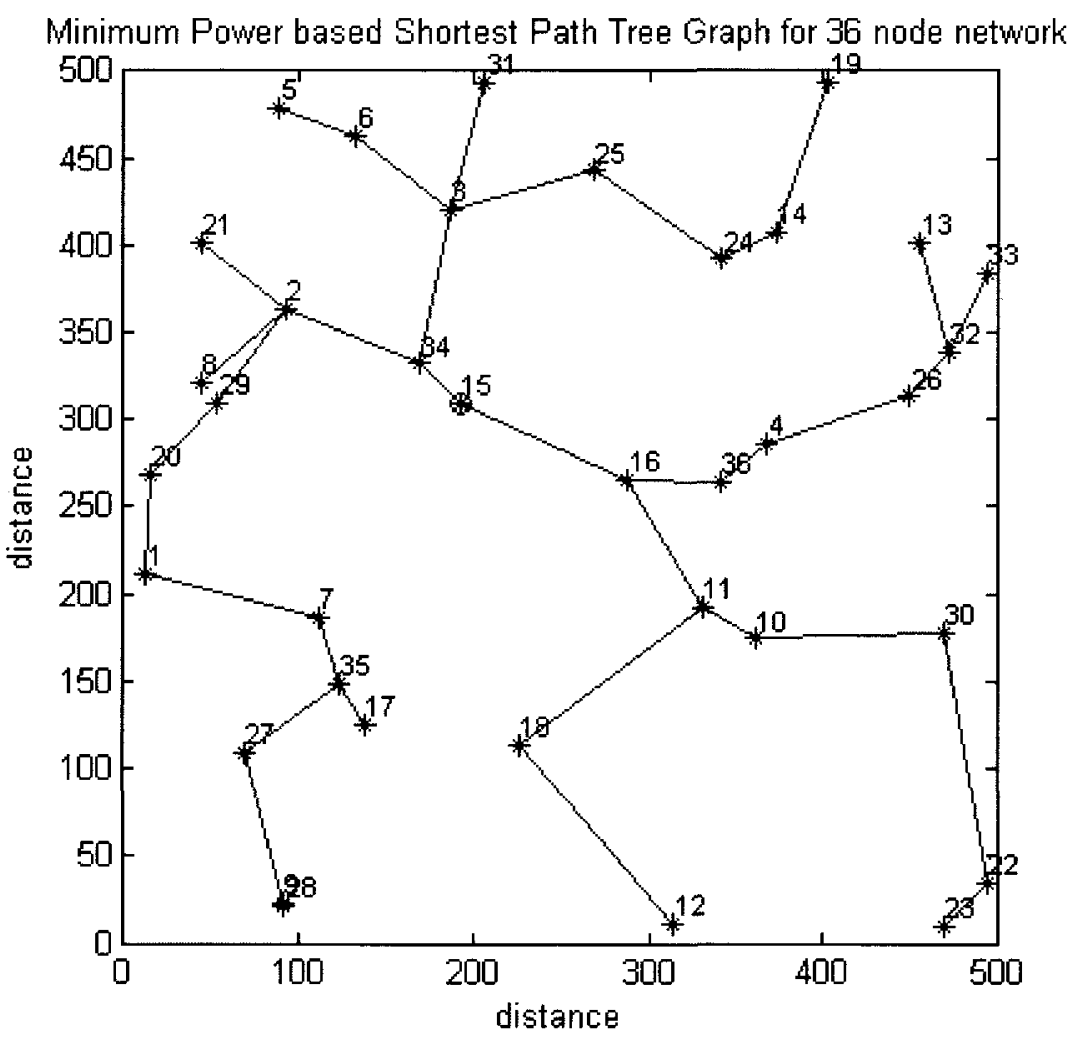

Figure C.6: MPSPT for $7^{\text {th }}$ RT 


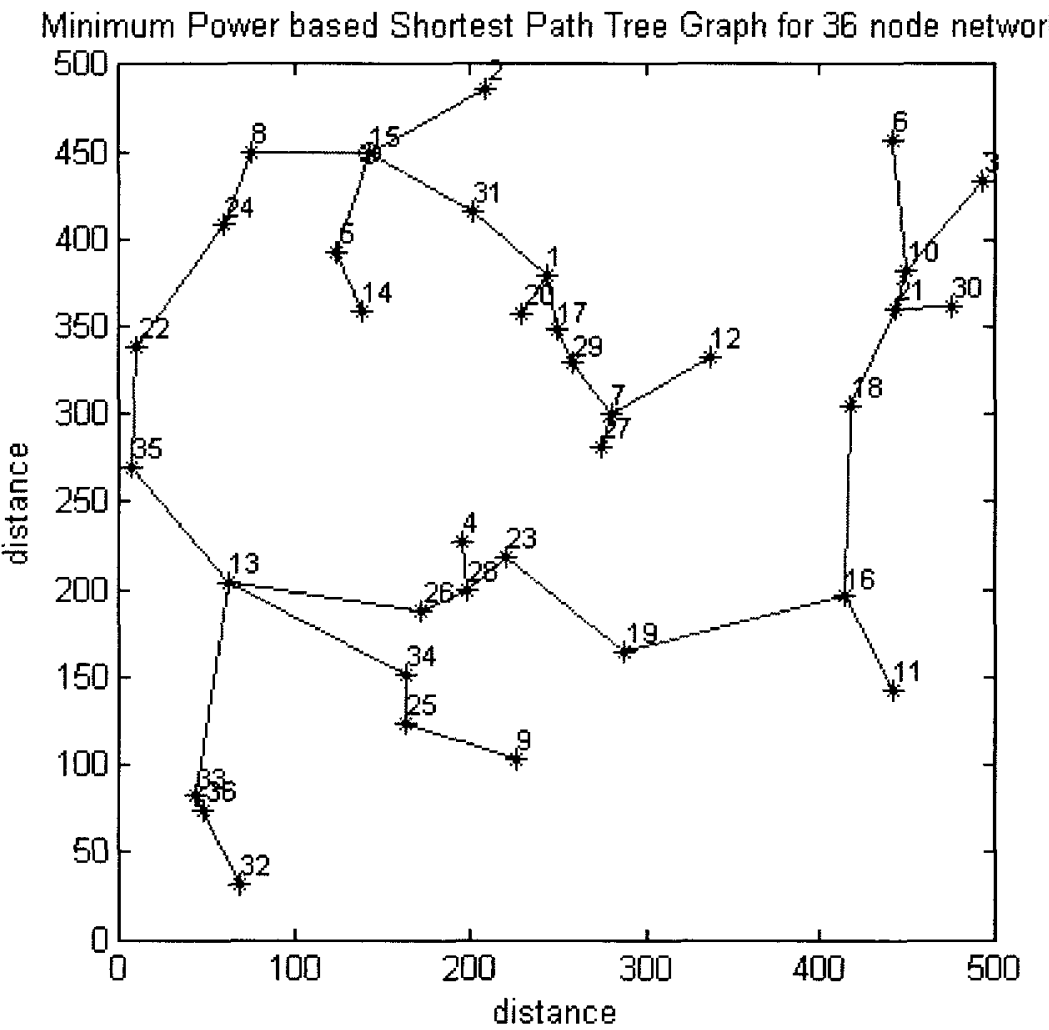

Figure C.7: MPSPT for $8^{\text {th }}$ RT

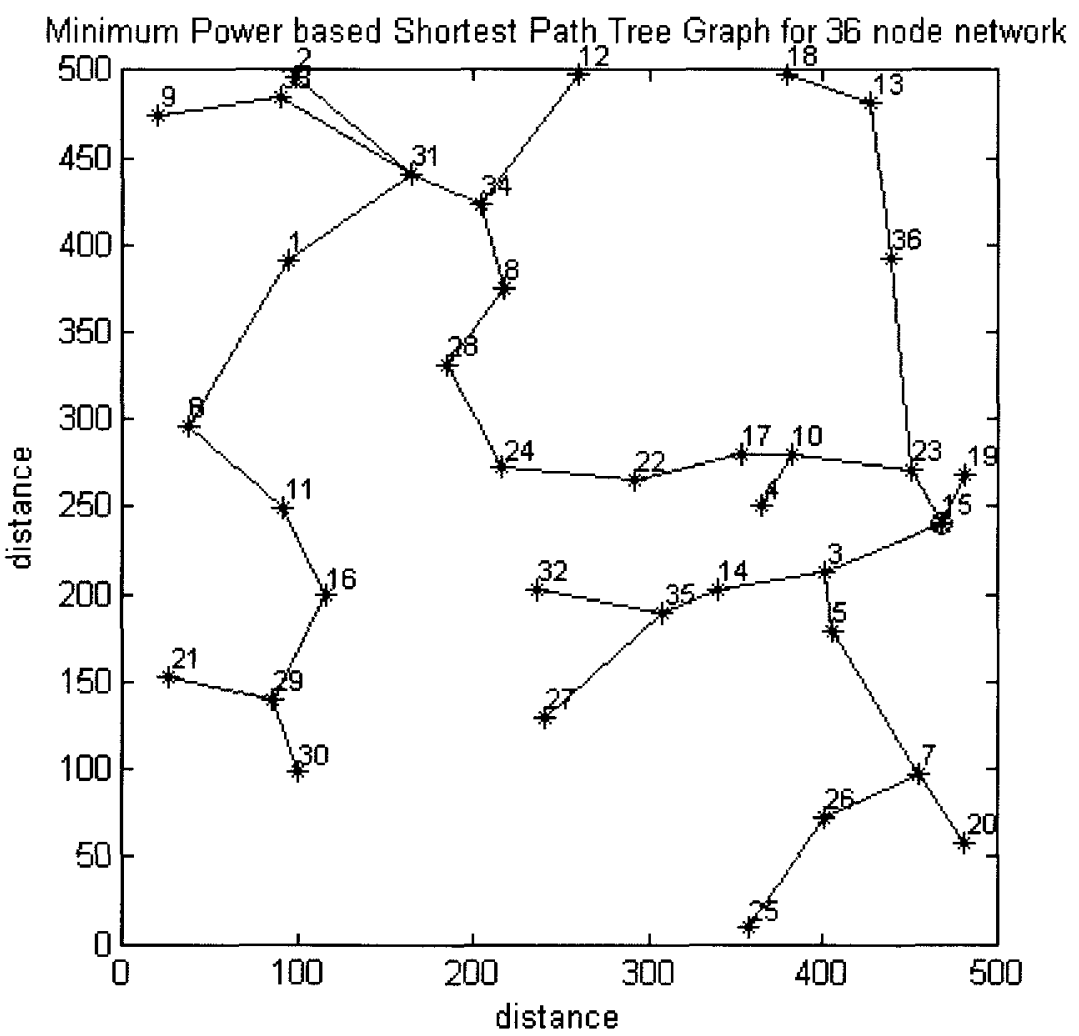

Figure C.8: MPSPT for $9^{\text {th }}$ RT 


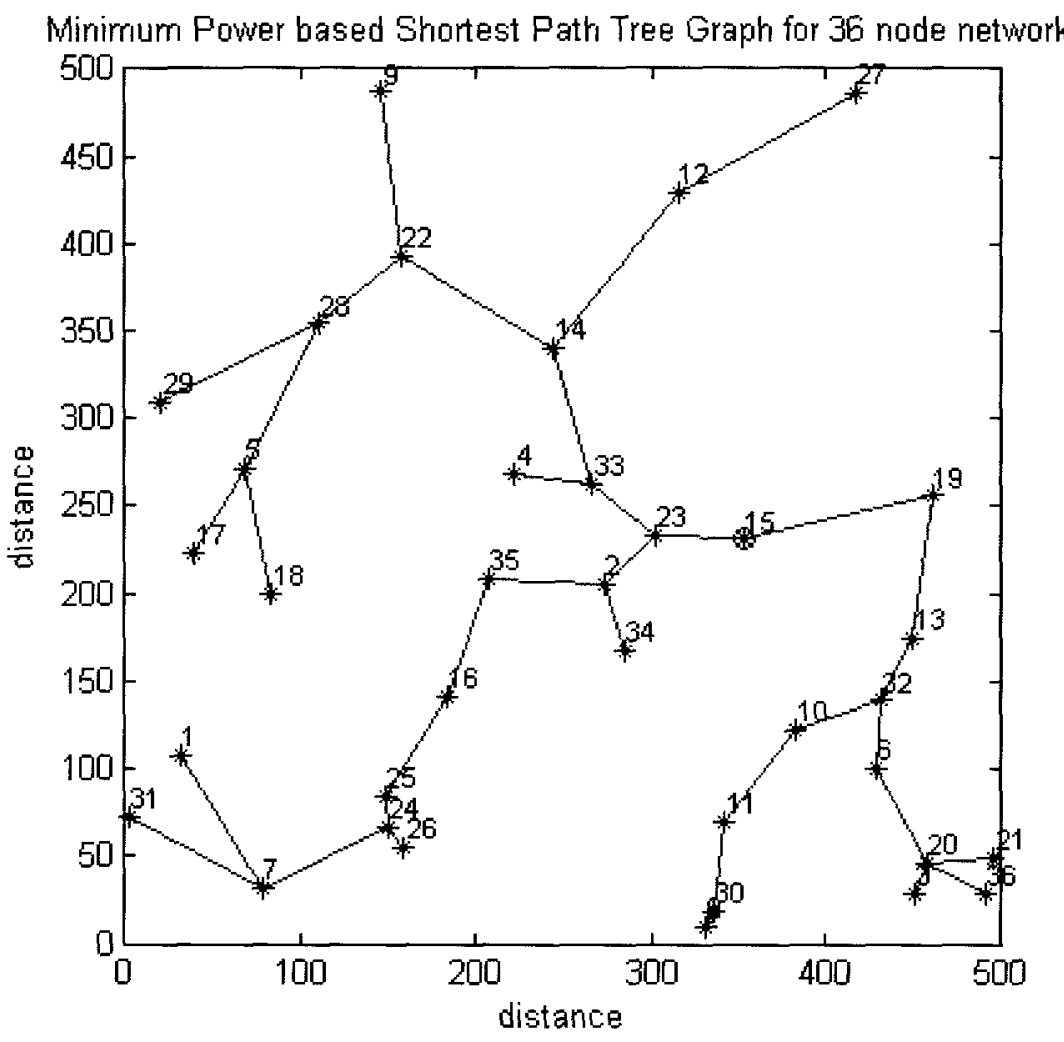

Figure C.9: MPSPT for $10^{\text {th }}$ RT

\section{C.2. MPSPT for Controlled Random Topology - 36-node Network}

\section{C.2.1. Seed 2}

For the $2^{\text {nd }}$-controlled random topology, the MPSPT with a MND of 4 resulting from Select 3 for less than 3 TCA for a 36-node network is shown in Figure C.10.

\section{C.2.2. Seed 3}

For the $3^{\text {rd }}$-controlled random topology, the MPSPT with a MND of 4 resulting from Select 3 for less than 3 TCA for a 36-node network is shown in Figure C.11.

\section{C.2.3. Seed 4}

For the $4^{\text {th }}$-controlled random topology, the MPSPT with a MND of 4 resulting from Select 3 for less than 3 TCA for a 36-node network is shown in Figure C.12.

\section{C.2.4. Seed 5}

For the $5^{\text {th }}$-controlled random topology, the MPSPT with a MND of 4 resulting from Select 3 for less than 3 TCA for a 36-node network is shown in Figure C.13. 


\section{C.2.5. Seed 6}

For the $6^{\text {th }}$-controlled random topology, the MPSPT with a MND of 4 resulting from Select 3 for less than 3 TCA for a 36-node network is shown in Figure C.14.

\section{C.2.6. Seed 7}

For the $7^{\text {th }}$-controlled random topology, the MPSPT with a MND of 4 resulting from Select 3 for less than 3 TCA for a 36-node network is shown in Figure C.15.

\section{C.2.7. Seed 8}

For the $8^{\text {th }}$-controlled random topology, the MPSPT with a MND of 4 resulting from Select 3 for less than 3 TCA for a 36-node network is shown in Figure C.16.

\section{C.2.8. Seed 9}

For the $9^{\text {th }}$-controlled random topology, the MPSPT with a MND of 4 resulting from Select 3 for less than 3 TCA for a 36-node network is shown in Figure C.17.

\section{C.2.9. Seed 10}

For the $10^{\text {th }}$-controlled random topology, the MPSPT with a MND of 4 resulting from Select 3 for less than 3 TCA for a 36-node network is shown in Figure C.18.

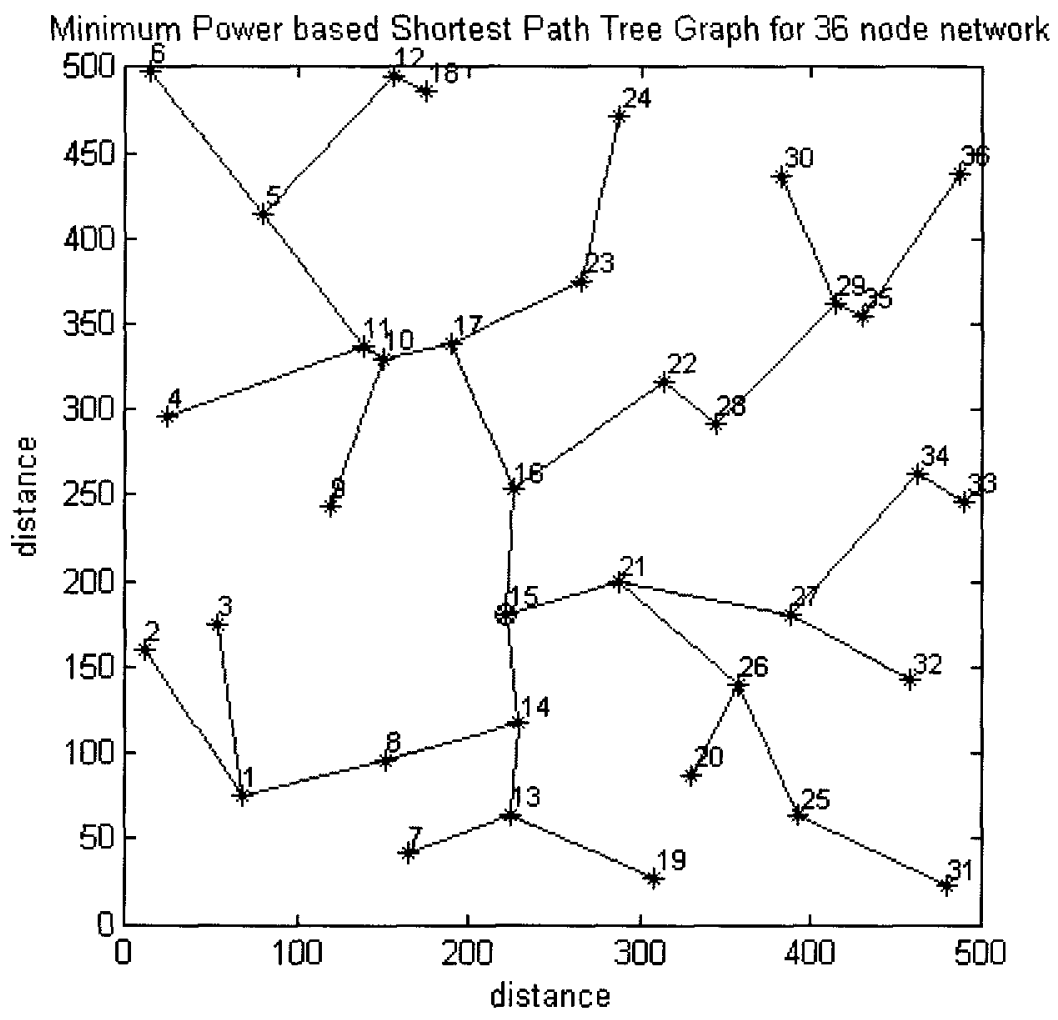

Figure C.10: MPSPT for $2^{\text {nd }}$ CRT 


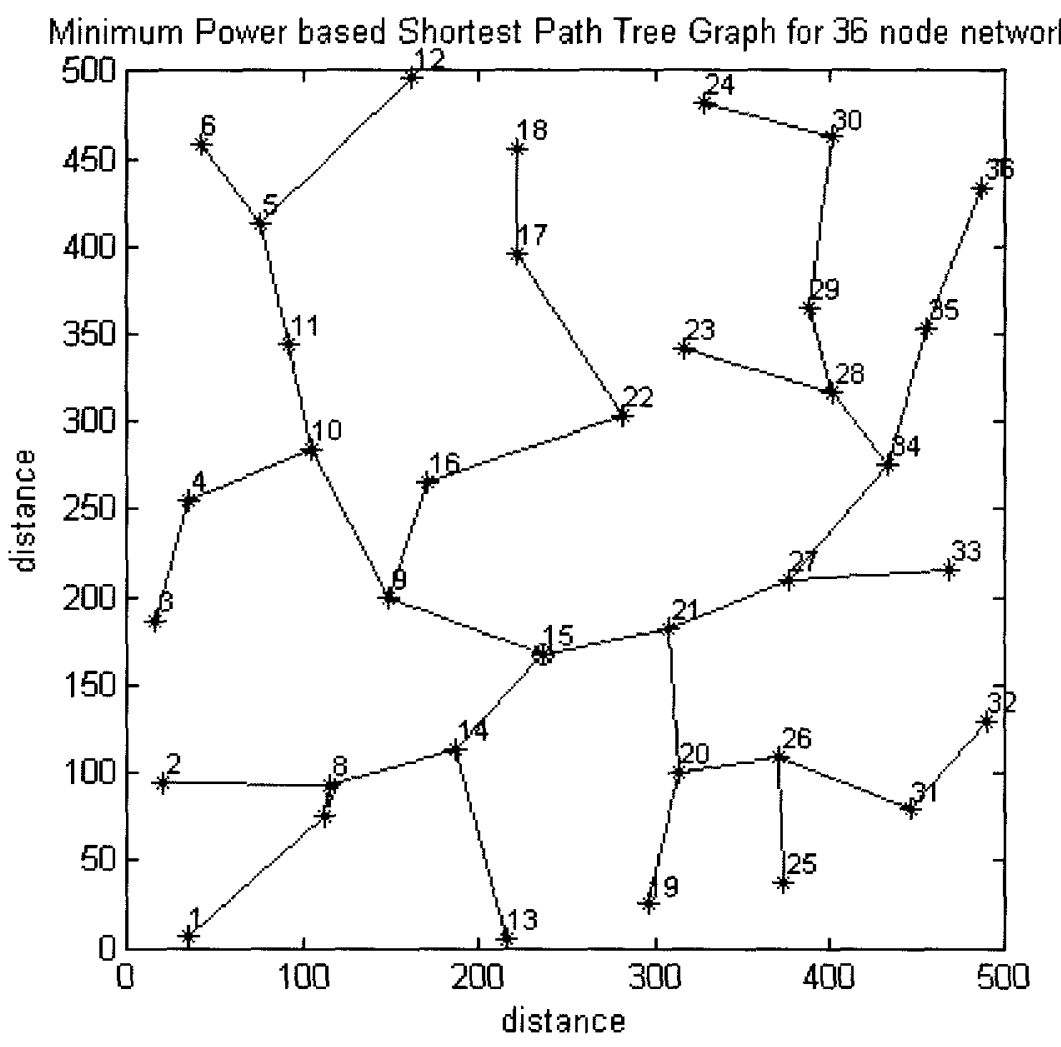

Figure C.11: MPSPT for $3^{\text {rd }}$ CRT

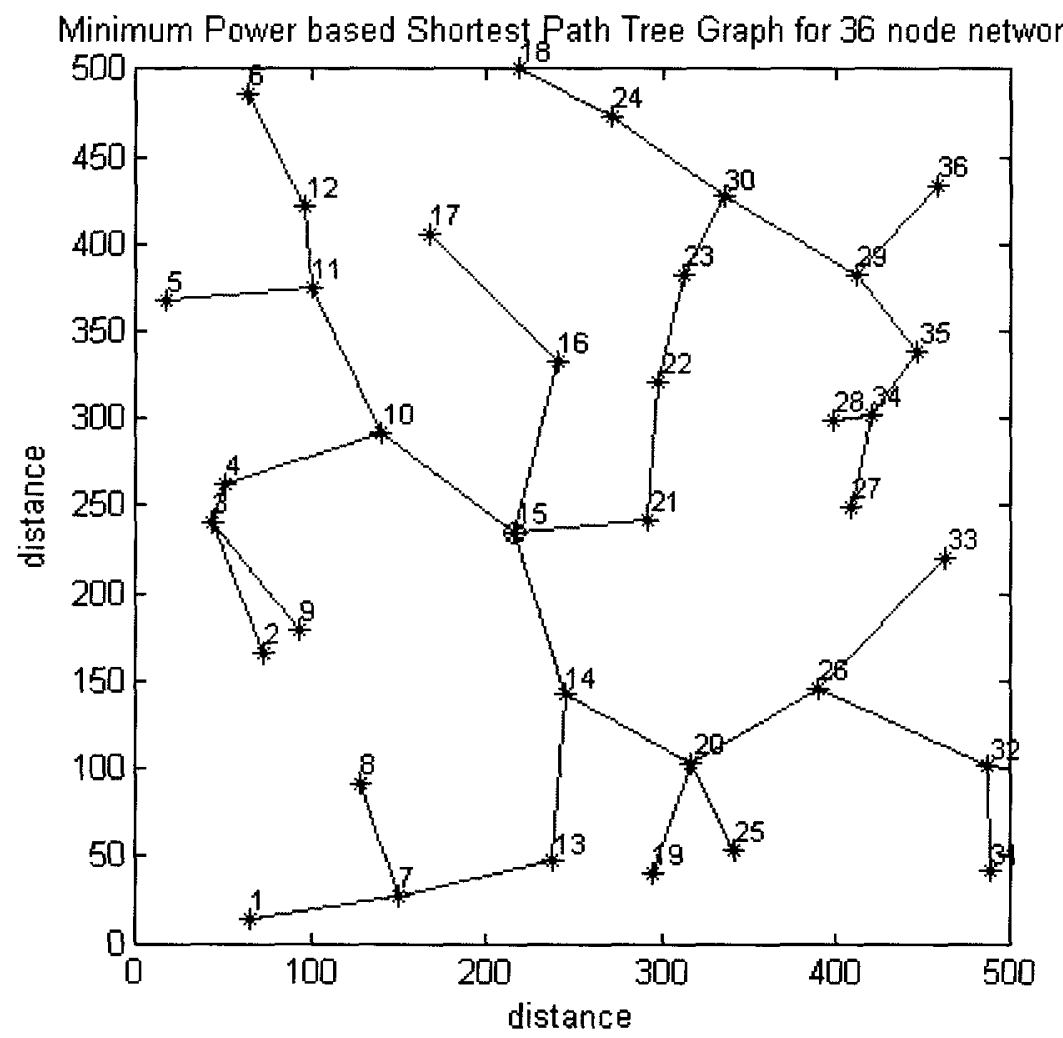

Figure C.12: MPSPT for $4^{\text {th }}$ CRT 
Minimum Power based Shortest Path Tree Graph for 36 node network

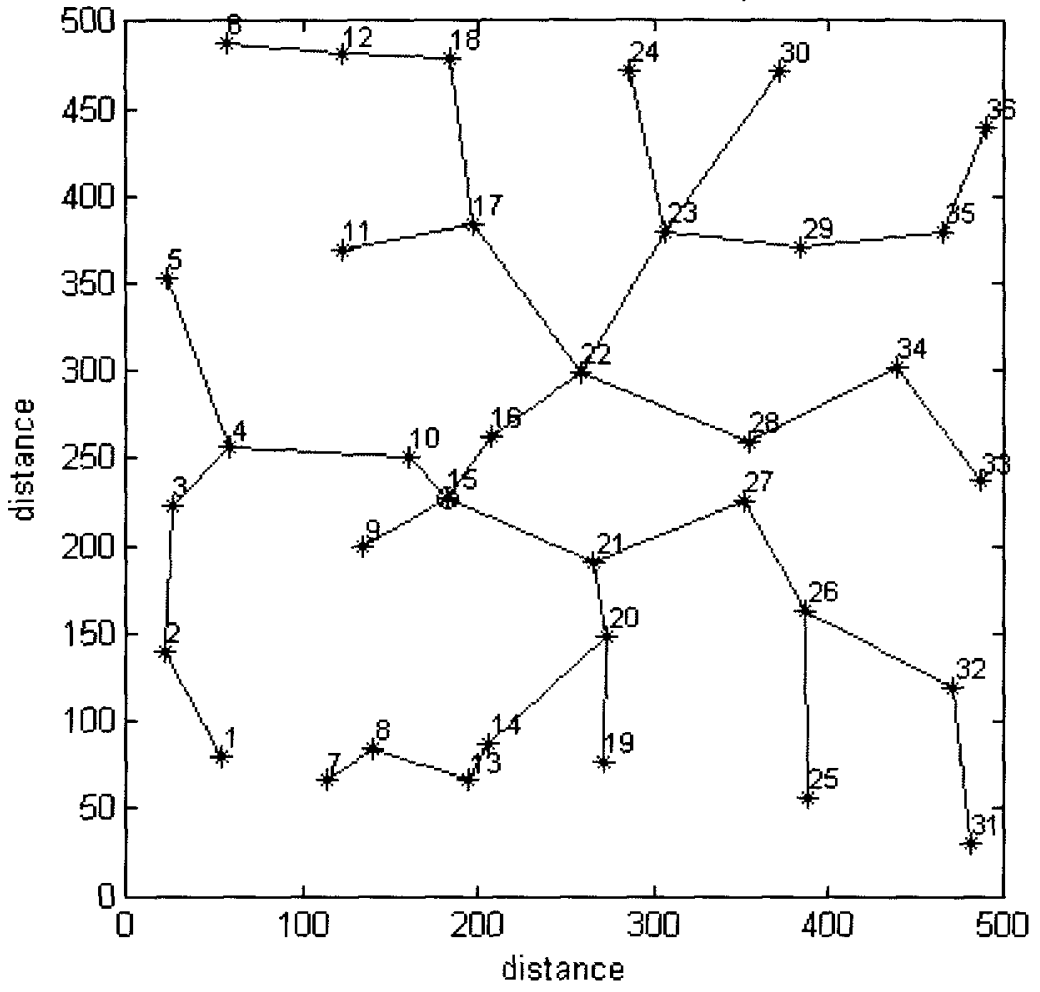

Figure C.13: MPSPT for $5^{\text {th }}$ CRT

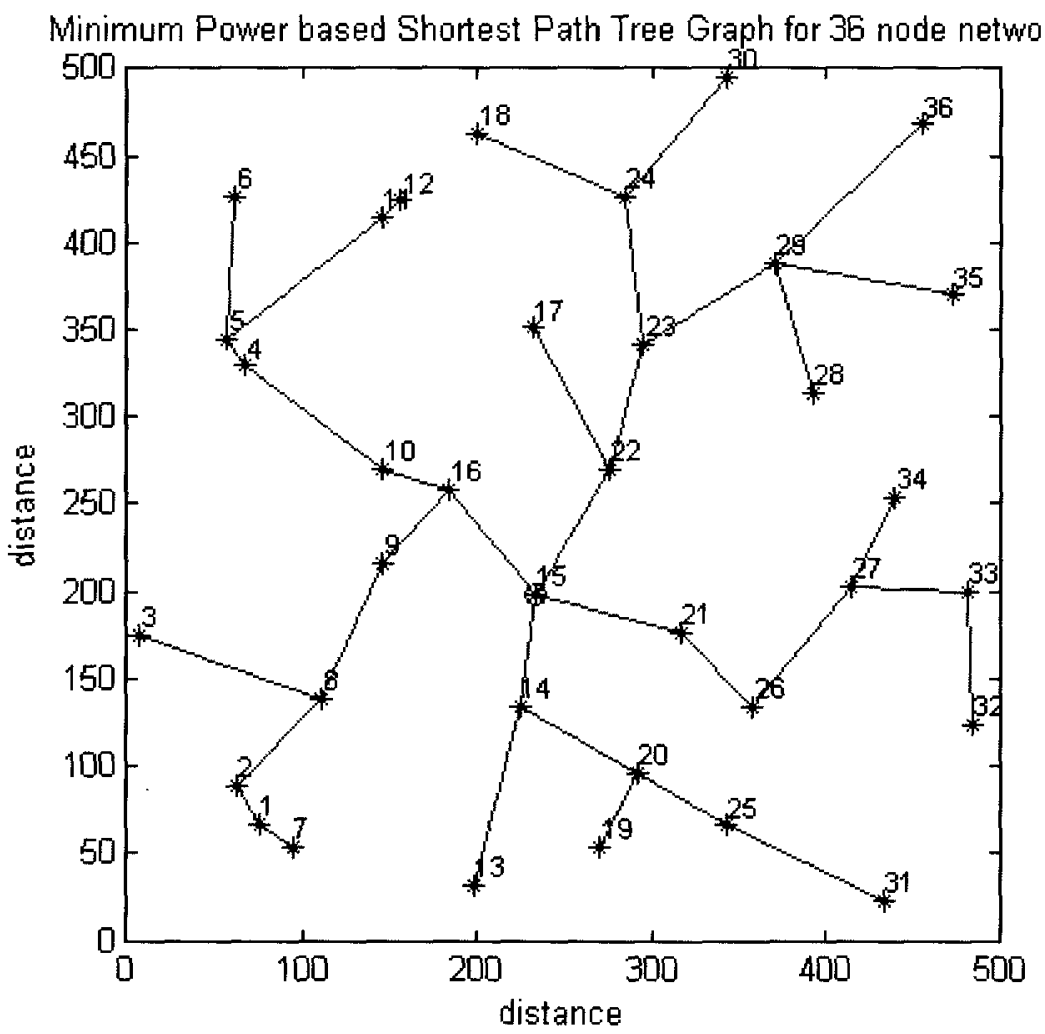

Figure C.14: MPSPT for $6^{\text {th }}$ CRT 


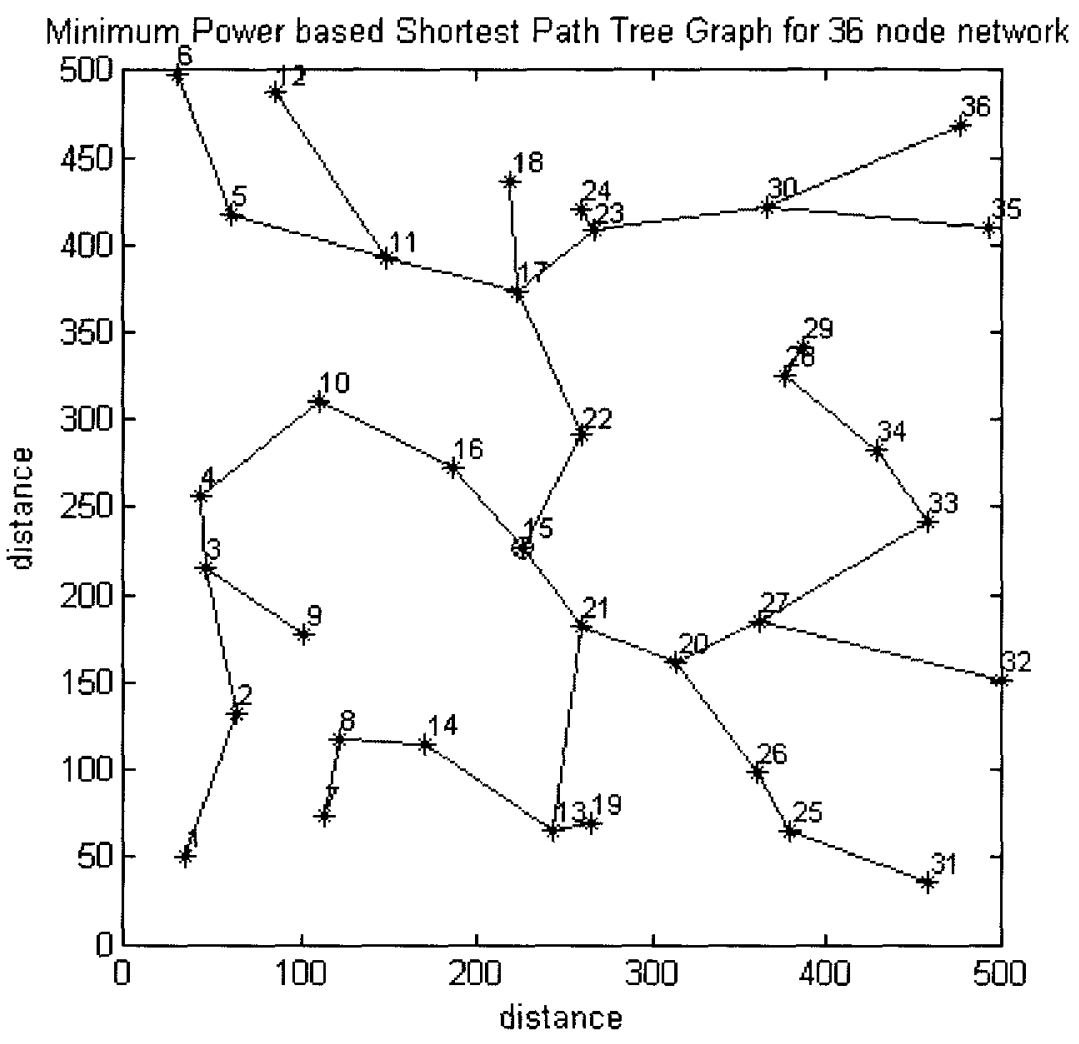

Figure C.15: MPSPT for $7^{\text {th }}$ CRT

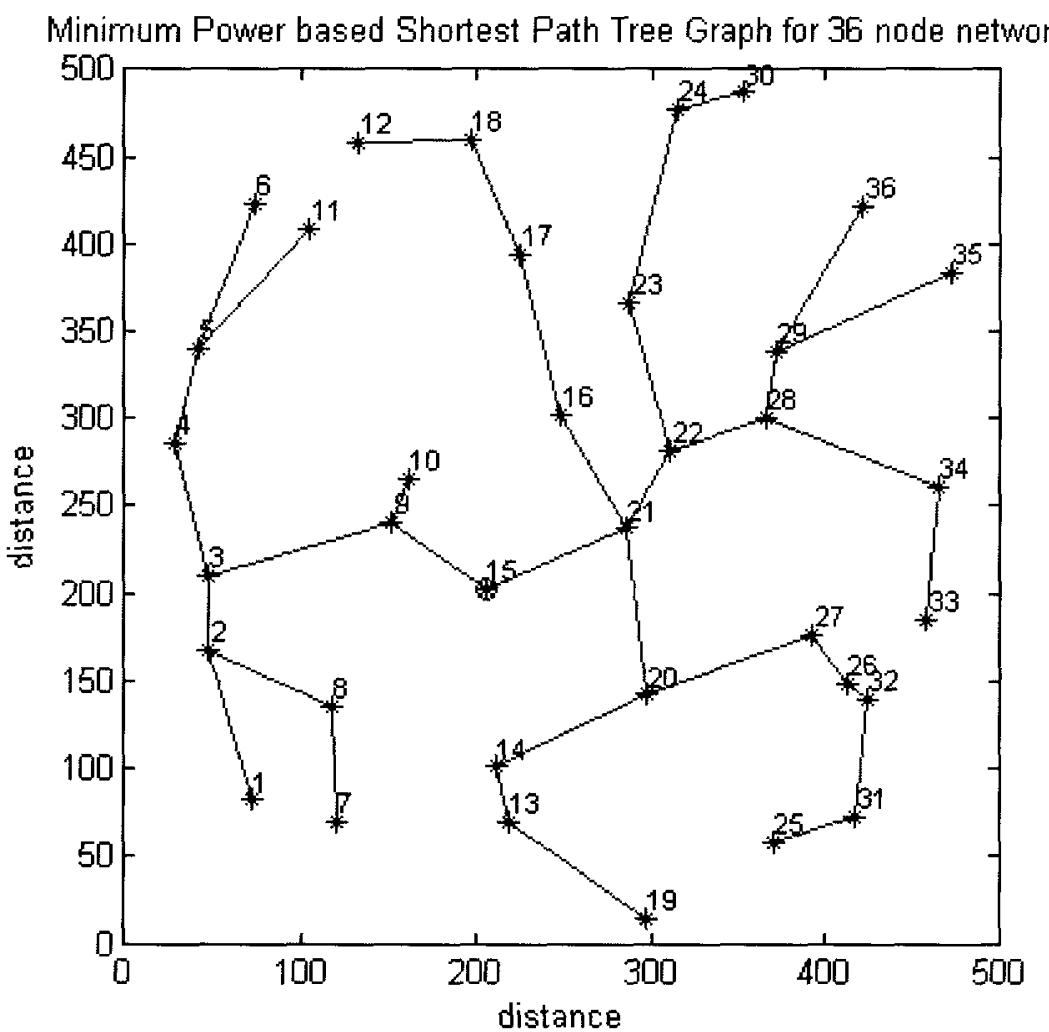

Figure C.16: MPSPT for $8^{\text {th }}$ CRT 


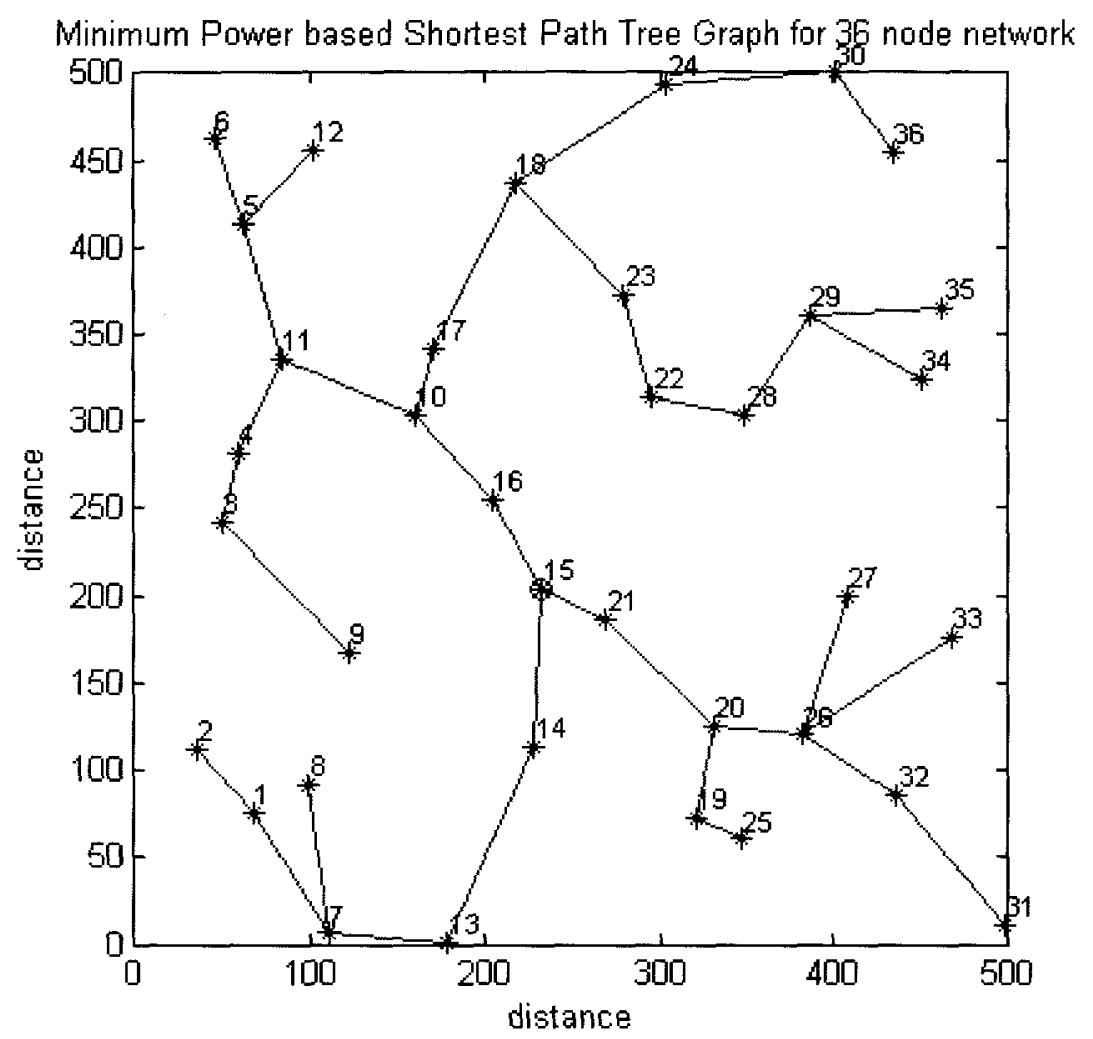

Figure C.17: MPSPT for $9^{\text {th }}$ CRT

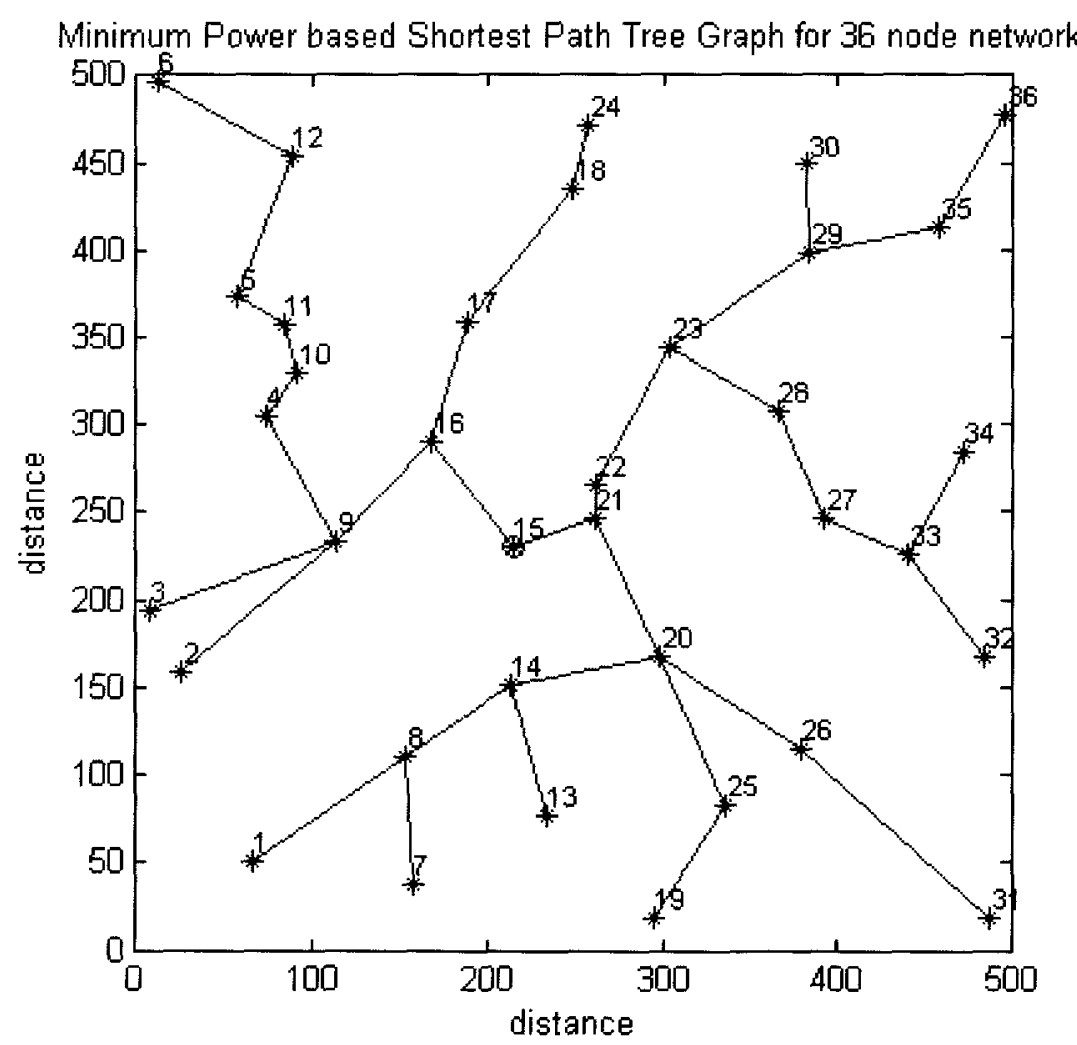

Figure C.18: MPSPT for $10^{\text {th }}$ CRT 


\section{C.3. MPSPT - 144-node Network}

\section{C.3.1. Random Topology}

For the random topology, the MPSPT with a MND of 4 resulting from Select 4 for less than 4 TCA for a 144-node network is shown in Figure C.19.

\section{C.3.2. Controlled Random Topology}

For the controlled random topology, the MPSPT with a MND of 4 resulting from Select 3 for less than 3 TCA for a 144-node network is shown in Figure C.20.

\section{C.3.3. Grid Topology}

For the grid topology, the MPSPT with a MND of 4 resulting from Select 1 for less than 1 TCA for a 144-node network is shown in Figure C.21.

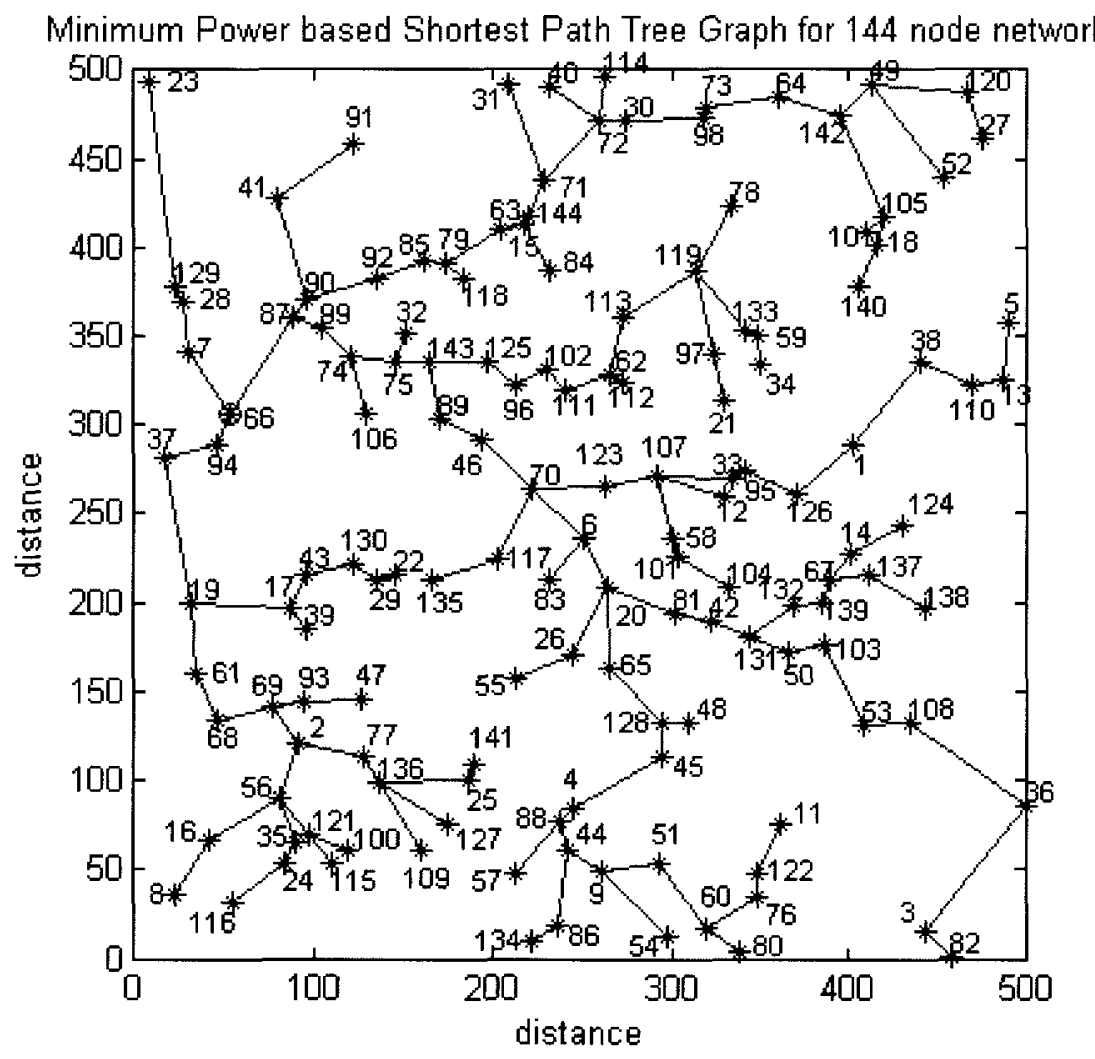

Figure C.19: MPSPT for RT - 144-node network 
Minimum Power based Shortest Path Tree Graph for 144 node network

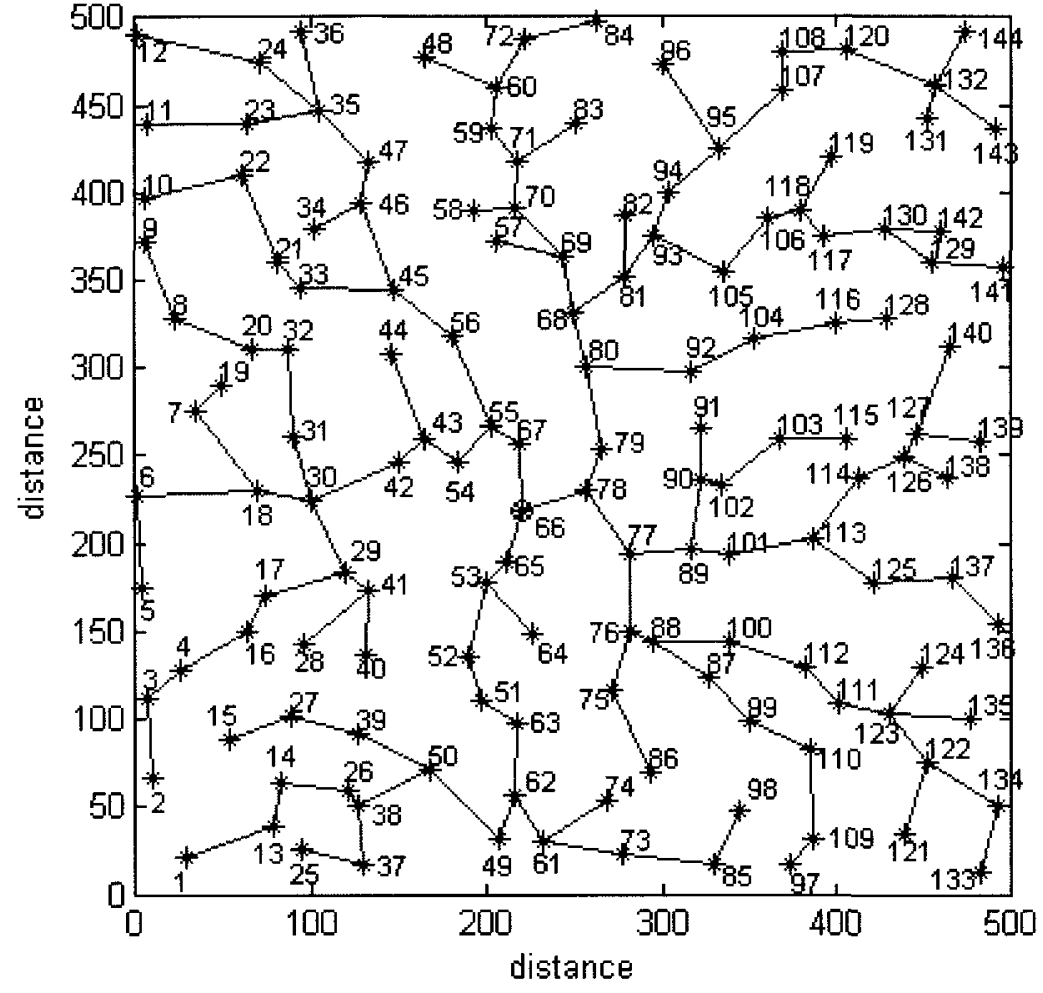

Figure C.20: MPSPT for CRT - 144-node network

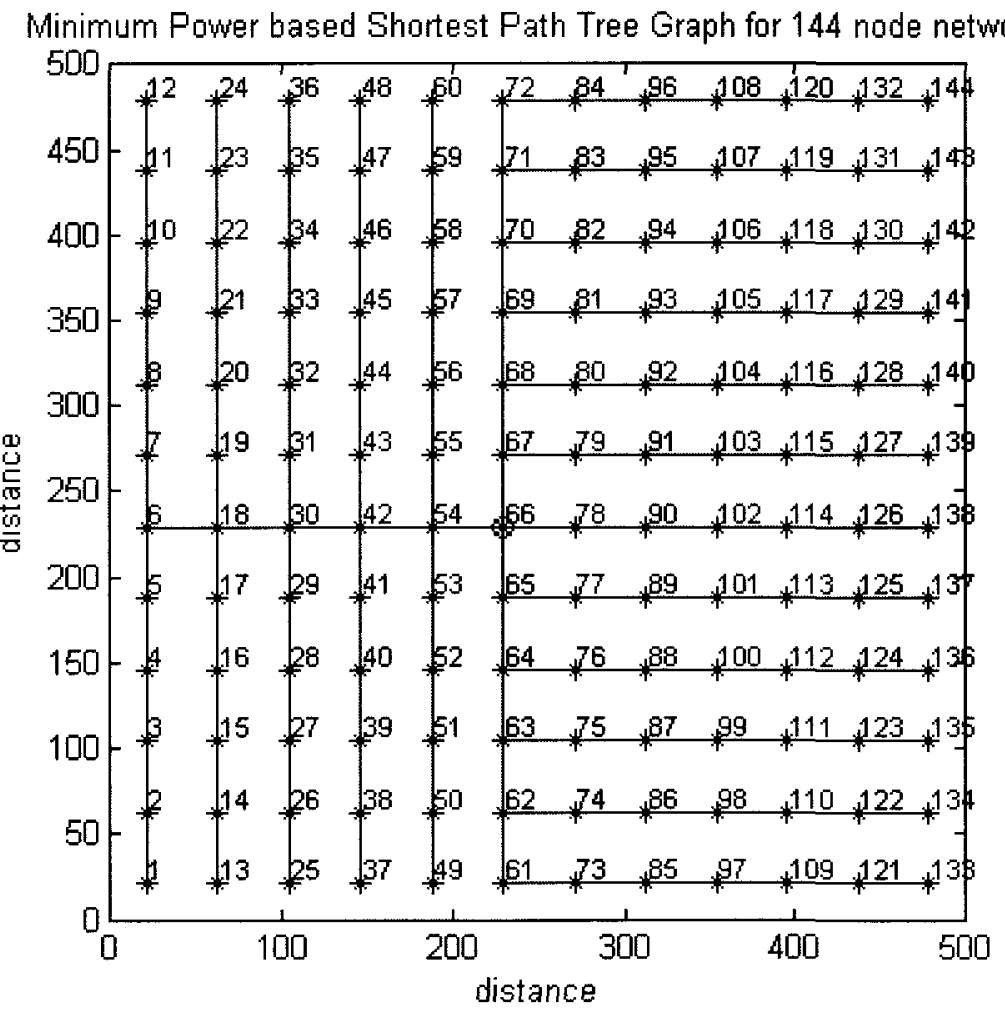

Figure C.21: MPSPT for GT - 144-node network 


\section{C.4. MPSPT - 100-node Grid Network}

The MPSPT with a MND of 4 resulting from Select 1 for less than 1 TCA for a 100-node grid network is shown in Figure C.22.

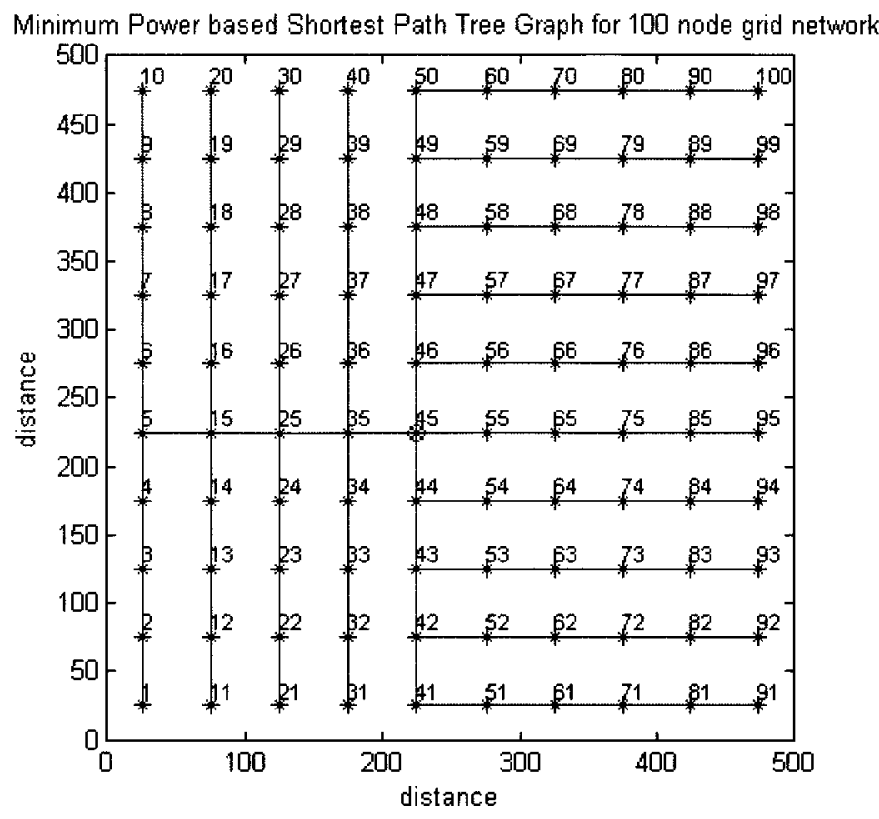

Figure C.22: MPSPT - 100-node grid network

\section{C.5. MPSPT - 30-node Grid Network}

The MPSPT with a MND of 4 resulting from Select 1 for less than 1 TCA for a 30 -node grid network is shown in Figure C.23.

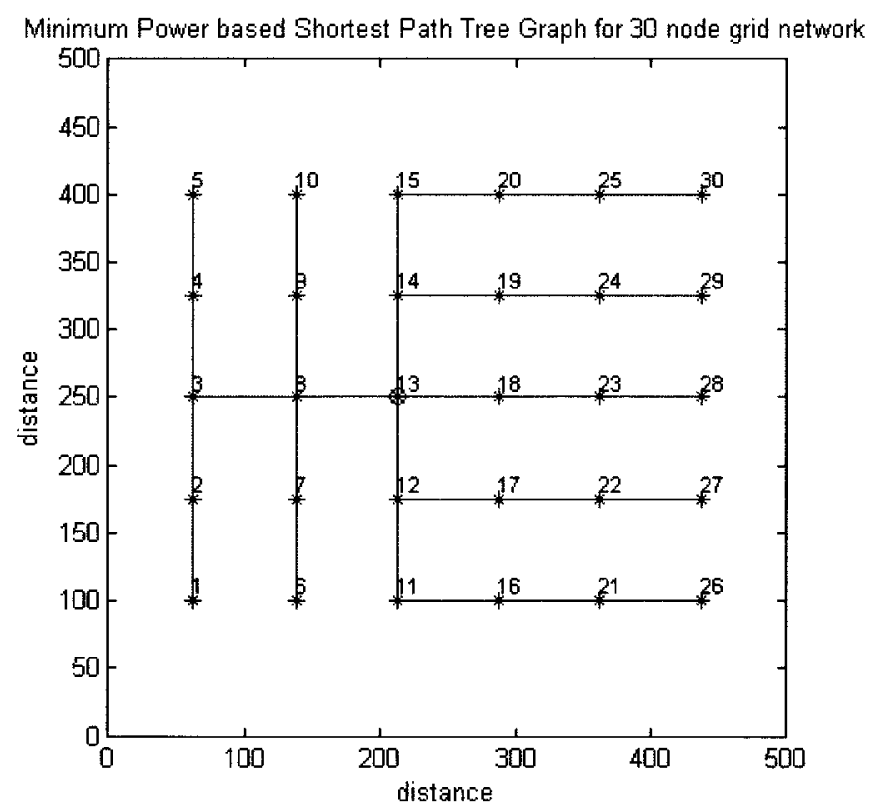

Figure C.23: MPSPT - 30-node grid network 